\title{
On maintaining successful treatment outcomes in interdisciplinary multimodal pain treatment
}

Citation for published version (APA):

Elbers, S. (2021). On maintaining successful treatment outcomes in interdisciplinary multimodal pain treatment. [Doctoral Thesis, Maastricht University]. Maastricht University. https://doi.org/10.26481/dis.20211117se

Document status and date:

Published: 01/01/2021

DOI:

10.26481/dis.20211117se

Document Version:

Publisher's PDF, also known as Version of record

\section{Please check the document version of this publication:}

- A submitted manuscript is the version of the article upon submission and before peer-review. There can be important differences between the submitted version and the official published version of record.

People interested in the research are advised to contact the author for the final version of the publication, or visit the DOI to the publisher's website.

- The final author version and the galley proof are versions of the publication after peer review.

- The final published version features the final layout of the paper including the volume, issue and page numbers.

Link to publication

\footnotetext{
General rights rights.

- You may freely distribute the URL identifying the publication in the public portal. please follow below link for the End User Agreement:

www.umlib.nl/taverne-license

Take down policy

If you believe that this document breaches copyright please contact us at:

repository@maastrichtuniversity.nl

providing details and we will investigate your claim.
}

Copyright and moral rights for the publications made accessible in the public portal are retained by the authors and/or other copyright owners and it is a condition of accessing publications that users recognise and abide by the legal requirements associated with these

- Users may download and print one copy of any publication from the public portal for the purpose of private study or research.

- You may not further distribute the material or use it for any profit-making activity or commercial gain

If the publication is distributed under the terms of Article $25 \mathrm{fa}$ of the Dutch Copyright Act, indicated by the "Taverne" license above, 


\section{on Maintaining Successful Treatment Outcomes in Interdisciplinary Multimodal Pain Treatment}




\section{COLOPHON}

Cover design: $\quad$ James Jardine | www.jamesjardine.nl

Layout: James Jardine | www.jamesjardine.nl

Print: $\quad$ Ridderprint | www.ridderprint.nl

ISBN: $\quad$ 978-94-93108-28-8

(c) Copyright Stefan Elbers, 2021, the Netherlands. All rights reserved. No part of this thesis may be reproduced or transmitted in any form or by any means, without the written permission of the author. 


\title{
On Maintaining Successful Treatment Outcomes in Interdisciplinary Multimodal Pain Treatment
}

\author{
Doctoral Thesis \\ to obtain the degree of $\mathrm{PhD}$ at Maastricht University \\ on the authority of the \\ Rector Magnificus Prof. dr. Rianne Letschert \\ and in accordance with \\ the decision by the College of Deans. \\ This thesis will be defended in public on \\ Wednesday $17^{\text {th }}$ of November 2021 at 16:00 hours
}

By

Stefan Elbers

Born on May 22, 1987

in Nijmegen 


\section{Promotor}

Prof. Dr. R.J.E.M. Smeets

\section{Co-promotoren}

Dr. H. Wittink

Dr. J.J.M. Pool

\section{Beoordelingscommissie:}

Prof. Dr. J.A. Verbunt (chair)

Dr. B.A. Brouwer

Prof. Dr. G. Crombez (Ghent University, Belgium)

Dr. B. Fullen (University College Dublin, Ireland)

Prof. Dr. M.L. Peters

This doctoral thesis was a collaboration between the Lifestyle \& Health research group (Department for Healthy and Sustainable Living) at the University of Applied Sciences Utrecht and the department of Rehabilitation Medicine (Care and Public Health Research Institute CAPHRI) at Maastricht University. The work presented in this thesis was funded by two SIA RAAK projects (2014-01-23P and RAAK.PUB05.002) and supported by the University of Applied Sciences Utrecht (HU). 



\section{TABLE OF CONTENTS}

01 General Introduction

02 Longitudinal Outcome Evaluations of Interdisciplinary Multimodal Pain Treatment Programmes for Patients With Chronic Primary Pain: A Systematic Review and Meta-Analysis

03 Living Systematic Reviews in Rehabilitation Science can Improve Evidence-Based Healthcare

04 The Effectiveness of Generic Self-Management Interventions for Patients with Chronic Musculoskeletal Pain on Physical Function, Self-Efficacy, Pain Intensity and Physical Activity: A Systematic Review and Meta-Analysis

05 Innovation in Pain Rehabilitation Using Co-Design Methods During the Development of a Relapse Prevention Intervention: Case Study

06 Exploring the Feasibility of Relapse Prevention Strategies in Interdisciplinary Multimodal Pain Therapy Programs: Qualitative Study

07 Mobile Health App (AGRIPPA) to Prevent Relapse After Successful Interdisciplinary Treatment for Patients With Chronic Pain: Protocol for a Randomized Controlled Trial

08 General Discussion

Summary

Nederlandse Samenvatting (Summary in Dutch)

Dankwoord (Acknowledgements)

Curriculum Vitae

List of Grants, Publications and Presentations 


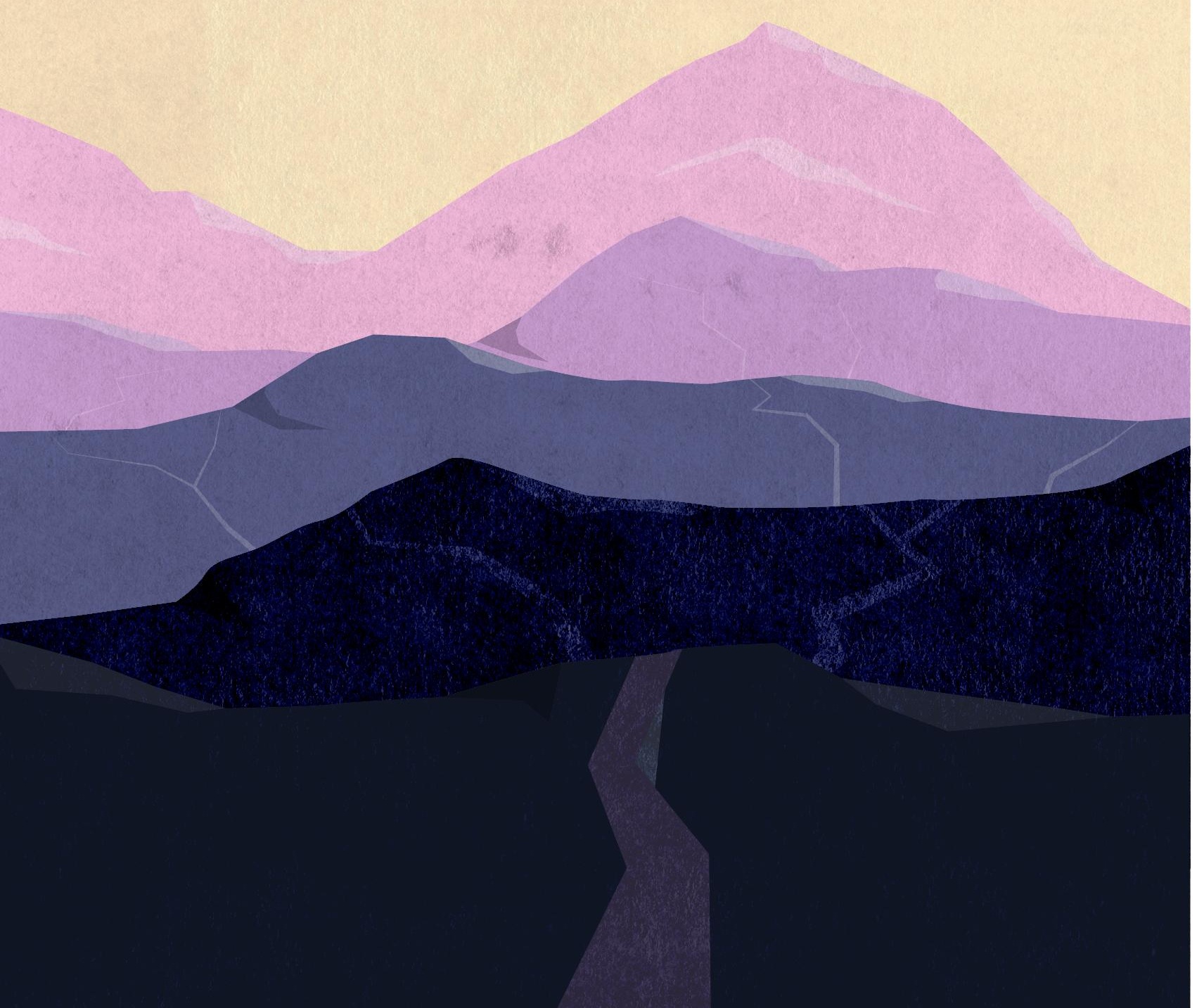




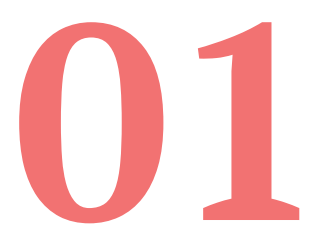

General Introduction 

Estimates suggest that some $30.3 \%(S D=11.7)$ of the global population suffers from chronic pain (Elzahaf et al., 2012). Although the duration and intensity vary from person to person, international surveys indicate that the negative effect on wellbeing is considerable. In a European survey including over 5000 people with chronic pain, the median Numeric Rating Scale (NRS) of pain intensity was 7 (range $=5-10$ ), and $78 \%$ of the sufferers reported a pain duration of at least 2 years (Breivik et al., 2006). The global impact of chronic pain is most evident in the estimates of the WHO burden of disease study where three musculoskeletal pain conditions were among the top 10 biggest contributors of years lived with disability (YLD). Low back pain leads the rankings and accounts for more YLD worldwide than conditions such as diabetes, major depression, and COPD (Blyth et al., 2019; Vos et al., 2017). These statistics indicate that on any given day millions of individuals worldwide have to deal with chronic pain, often for years on end. Unsurprisingly, many of these report comorbidities such as depression, anxiety, fatigue, and an inability to fulfil their social roles (Breivik et al., 2006; Pitcher et al., 2019), leading to a complex cluster of interacting problems that affect social, mental and physical functioning (Doleys, 2017).

\section{DEFINITION AND FUNCTION OF PAIN}

The International Association for the Study of Pain (IASP) defines pain as "an unpleasant sensory and emotional experience associated with, or resembling that associated with, actual or potential tissue damage" (2021). When pain is persisting or recurring for at least three months - which is generally beyond the required time for tissue to heal - it is referred to as chronic pain (Treede et al., 2019). Despite its unpleasantness, the ability to feel pain is an adaptive trait that is crucial for survival (Williams, 2016). This becomes clear when noxious stimuli threaten the body's integrity. Nearby nociceptors detect the incoming danger and, when sufficiently stimulated, activate peripheral nerve fibers that transmit the signal to higher-order brain regions. Given that the signal is strong enough to capture the attention of these regions and that the situation is evaluated as sufficiently threatening, a pain experience emerges (Brodal, 2017). This experience interrupts ongoing cognitive processes, such as attentional performance, and motivates the individual to deal with the threat (Moore et al., 2019; Vlaeyen \& Crombez, 2020). In these situations, pain serves as a symptom - a warning of a potential threat against the body's integrity. The importance of this mechanism for survival is best illustrated in conditions that are characterized by congenital nociceptor deficiency, such as hereditary sensory and autonomic neuropathy type V, which prevents people experiencing pain after injury (Weisman et al., 2019). This condition leads to many accidental, unrecognized, and untreated self-inflicted wounds, fractures, and cuts, as well as premature death (Erdil et al., 2012; Yozu et al., 2016). 


\section{ACUTE PAIN VERSUS CHRONIC PAIN}

From a neuroscientific perspective, the experience of pain is most likely to emerge from an activated pattern of functionally connected cortical areas (Brodal, 2017). This network reaches down via the thalamus, the spinothalamic tract, and peripheral nerve fibers to the nociceptors throughout the body (Brodal, 2017). At numerous levels in this sensory system, the amplitude of the signal can be modified, thereby influencing the pain response (Kosek et al., 2016). In many instances, this flexibility is regarded as an adaptive process (Clauw et al., 2019; McCarberg \& Peppin, 2019; Tabor \& Burr, 2019). For example, temporary local peripheral sensitization caused by inflammation processes in injured tissues promotes vigilance and behaviours protective of vulnerable tissue during the process of healing (Basbaum et al., 2009). However, alterations in sensitivity independent of tissue damage allow for the possibility of persistent pain without an underlying pathological substrate in local tissues or even the activation of peripheral nociceptors. In these instances, pain loses its warning function and shifts from being a symptom to a disease in its own right. This is reflected in the ICD-11 framework, where pain can be classified as a symptom related to an underlying disease, but also as a disease in itself (Treede et al., 2015, 2019). Importantly, the existence of sensitizing mechanisms across the nociceptive system does not imply that all chronic pain is independent of local pathology. The multiple parenting option of the ICD-11 provides the option to combine multiple categories to classify mixed conditions accordingly.

Although neuroscientific studies have provided empirical support for modulating mechanisms across the nociceptive network, the experience and consequences of chronic pain cannot be completely understood through this paradigm (Doleys, 2017). As many authors have discussed, it is not a nervous system that suffers from chronic pain, but a person in interaction with their social context (Doleys, 2017; Eccleston, 2018; Hruschak \& Cochran, 2017; Thacker \& Moseley, 2012). It is therefore important also to consider the transition from acute to chronic pain through psychological and social lenses.

Many studies in the psychological domain have emphasized the importance of associative learning processes in the transition from acute to chronic pain (e.g. Moseley \& Vlaeyen, 2015; Vlaeyen et al., 2016). A pain experience demands attention, often interrupting ongoing physical and psychological processes (Berryman et al., 2013; Moore et al., 2019). What follows is an evaluation where people attempt to make sense of the potential threat and decide an appropriate protective response (Seymour, 2019). This process involves making inferences from sensory input (both interoceptive and salient contextual information), combined with previously learned cognitive and emotional associations, such as beliefs and fears (Phelps et al., 2021). Irrespective of the type of response at a particular moment, 
associative learning does take place in these situations, often reinforcing the relationships between pain, certain activities, fear of injury, and impulses to initiate protective behaviours (Tabor \& Burr, 2019). Through generalization within this associative network, for example, by extrapolating the threat value of specific movement actions (such as lifting heavy objects at work) towards general activities (such as lifting anything), chronic pain increasingly interferes with daily life. Over time, the interrupting effect of pain on activities, the associated negative affect, and the impulses towards protective behaviours at the cost of other valued activities increasingly impact a person's wellbeing and identity. People who valued themselves as parents, partners, friends and colleagues, increasingly regard themselves as patients with pain (Toye et al., 2017). This dominance of pain in the lives of patients is not only corroborated by qualitative studies, where patients report an impoverished and constricted life, but also by experiments where patients with chronic pain have an attentional bias towards pain-related stimuli, compared with healthy controls (Todd et al., 2018; Toye et al., 2017).

It is important to realize that these psychological processes do not stand alone, but that they interact with noxious input and peripheral processing (Phelps et al., 2021; Woller et al., 2017). Consequently, the ongoing hypersensitivity and persistent pain that result from sensitization of the pain network will influence how pain is perceived and appraised over time (Brodal, 2017; Phelps et al., 2021).

Although the influence of social context has received less attention in both research and treatment programmes, environmental factors, including work, family relationships, and health care provider perceptions, have a considerable influence on chronic pain and its impact on wellbeing (Darlow et al., 2013; Hruschak \& Cochran, 2017; Mescouto et al., 2020). Pain-related disability impairs relationships with co-workers, friends, significant others, and children. Furthermore, the culturally dominant view that pain signals injury often reinforced by health care providers (e.g. Nijs et al., 2013) - can shape beliefs towards a biomedical understanding of their pain. For example, a thematic analysis of readers' comments to an article in a national newspaper in the United Kingdom that presented a biopsychosocial view of pain revealed themes such as the need for MRI scans to diagnose chronic pain, the danger of exercising with pain, and the idea that people who prescribed exercise were not taking the problem seriously (McCarthy \& Yeowell, 2017). This is problematic because a biomedical perspective towards chronic pain is related to ineffective coping strategies, worrying, and increased disability (Eccleston \& Crombez, 2007). 
Taken together, the system of biomedical, psychological, and social factors provides a comprehensive understanding of how an inherently adaptive function can become a disease in itself, leading to severe disability and an inability to fulfil social roles and personally valued activities.

\section{LIVING WITH CHRONIC PAIN}

\section{Psychosocial Coping Variables}

The previous section showed how a cluster of interrelated biopsychosocial factors contributes to the development of chronic pain. For patients who live with pain for years on end, it is also important to understand how to manage the impact of chronic pain on day-to-day activities. Although subgroups in coping patterns have been observed (e.g. Hasenbring et al., 2020), the multitude of identified personal and social factors that influence coping with pain suggest unique behavioural responses and pain management strategies for each patient (O'Sullivan et al., 2018). An example of these factors is the personality trait of optimism (i.e. a general tendency towards favourable expectations concerning their future), which is positively related to more flexible goal-adjustment when confronted with goal-conflict situations (Ramírez-Maestre et al., 2019). Other factors that have been observed to positively influence coping are undertaking physical activity, selfcompassion, and acceptance of pain (Åkerblom et al., 2015; Edwards et al., 2019; Edwards et al., 2016). As regards coping with pain, an important vulnerability factor is the amount of negative affect, such as distress, felt by the patient (Edwards et al., 2016). Difficulties in regulating these negative emotions may result in symptoms of depression or anxiety, which negatively influence long-term outcomes (Edwards et al., 2016; Karoly, 2020). The social environment also influences coping with chronic pain (Edwards et al., 2016). For example, global social support is positively associated with pain coping, unless this support encourages pain-contingent behaviours or excessive resting (Jensen et al., 2011).

\section{Goals and Goal-Conflict}

In addition to the various psychosocial variables that influence pain coping, it is also important to consider the continuous impact of chronic pain on goal pursuit in general. Goals are defined as mental representations of desired future outcomes (Ramnerö \& Törneke, 2015). They serve as a reference standard to guide current actions towards these envisioned outcomes. Goals do not operate in a vacuum but are embedded in a hierarchical motivational network where higher-order values are associated with goals and concrete actions (Carver \& Scheier, 2017; Höchli et al., 2018). Given the limitations of a humans attention span, goals are not continuously active. Instead, they can be retrieved from 
memory by salient contextual cues and attentional processes, such as the sight of people running in a park that triggers the goal to exercise. Goal conflicts arise when multiple incompatible goals are active at the same time (Boudreaux \& Ozer, 2013). For patients with chronic pain, goal conflicts often occur and are related to situations where pain interrupts valued activities (Claes et al., 2018). At these moments, the strength of each particular goal, determined by factors such as motivation, affects the choice of whether to prioritize continuation of an activity or engage in actions related to pain control. These continuous conflicts between valued activities and protective behaviours to control pain or avoid harm pose a constant challenge for individuals with pain. Resolving these conflicts demands cognitive capacity and motivational resources (Baumeister 2014) and, even when people choose to pursue valued activities, the interrupting effect of pain often results in decreased performance (Moriarty et al., 2011).

\section{Variation in Clinical Manifestations}

The sections above illustrate that chronic pain not only has a considerable impact on wellbeing but also involves a network of interacting biopsychosocial factors that allow for many possible clinical manifestations. Indeed, this corresponds to the large variations in baseline measures of distress and depression that have been observed in patients who are about to start treatment (Morley et al., 2013). This justifies a comprehensive approach towards assessment and treatment, tailored to the specific situation of each individual.

\section{TREATMENT APPROACHES FOR PATIENTS WITH CHRONIC PAIN}

\section{Interdisciplinary Multimodal Pain Treatment}

The impact of chronic pain on daily life activities, valued goals, and one's identity, described previously, explains why many patients seek medical assistance. Although there is no therapeutic agent available that directly modifies pain intensity, the abundance of modifiable psychosocial variables offers a pathway towards reduced disability and increased wellbeing (Edwards et al., 2019; O’Sullivan et al., 2018). Consequently, most pain management programmes are focused on optimizing self-management, wellbeing and daily life functioning, irrespective of pain (Williams et al., 2020). For patients with substantial disability and loss of quality of life, such therapies are often delivered by multiple health care providers in comprehensive pain management programmes. These programmes originated in response to the inability to provide care for WWII veterans with chronic pain, who experienced a maze of referrals, without a clear diagnosis or adequate treatment. In the 1950s, Dr John Bonica advocated better coordination between disciplines, 
which resulted in the start of the first pain management programme in the United States in 1978 (Gatchel et al., 2014; IASP, 2021b; Kaiser et al., 2017). Over time, facilities that offered these programmes continued to integrate new findings, such as the biopsychosocial model (Gatchel et al., 2014), and adapted to constraints of local policy-making and health care systems (Kaiser et al., 2017). At present, these types of treatment have evolved into interdisciplinary multimodal treatment (IMPT) programmes. These interventions feature interdisciplinary teams of health care providers with different professional backgrounds, which coordinate their services throughout treatment and work within one facility. The various disciplines share a biopsychosocial understanding of chronic pain (Kaiser et al., 2017). Activities always include active patient involvement and often consist of a combination of education, exercise, and (cognitive) behavioural approaches, including pain reconceptualization, psychological flexibility, extinction of pain-related fear, and gradual increase in valued daily life activities (McCracken \& Vowles, 2014; Vlaeyen, et al., 2016).

Despite these similarities, there are signs that IMPT programmes vary considerably concerning patient inclusion criteria, dose, and content (Waterschoot et al., 2014). Crucially, choices regarding duration or the inclusion of specific treatment modalities are often not reported, obscuring interpretation of the results (Morley et al., 2013).

A second problem is that systematic reviews indicate a positive, but limited, effect of IMPT programmes, compared to control conditions, at follow-up (Chou et al., 2017; Kamper et al., 2015; Williams et al., 2020). A possible explanation for these small-to-medium effects is the difficulty of adhering to treatment principles over time. This hypothesis mirrors current ideas regarding the difficulty in successfully promoting sustainable change in health behaviours through interventions (Wood \& Neal, 2016). Further, in contrast to other chronic health conditions, this problem of potential relapse has been a neglected topic for decades in the field of pain management (Backs-Dermott et al., 2010; Martins et al., 2020; Morley, 2008; Turk \& Rudy, 1991). A possible explanation for this is the difficulty in delineating clear endpoints to define relapse in these type of interventions. By definition, IMPT programmes include various modalities that aim to achieve and maintain optimal wellbeing via multiple routes (Gatchel et al., 2014; Kaiser et al., 2017). As a consequence, these programmes are evaluated by a broad set of measurement instruments that cover a wide range physical, psychological and social health outcome domains (Kaiser et al., 2018). This is different from treating conditions where the focus on more concrete outcomes or behaviours allows for more precise criteria of relapse, such as substance abuse (return to uncontrolled usage), obesity (weight regain) and depression (presence of at least five depressive symptoms) (Backs-Dermott et al., 2010, Melemis, 2015; Martins et al., 2020). 


\section{Self-Management Interventions}

One cannot not manage...The only question is how one manages. (Lorig \& Holman, 2003).

Self-management has been defined as the everyday management of chronic conditions during the course of a disease (Allegrante et al., 2019). It generally involves behavioural or medical tasks to deal with the condition, but also coping with emotions and managing valued social roles (Lorig \& Holman, 2003). Generic self-management interventions are a less comprehensive alternative compared to IMPT programmes. They do not target diseasespecific mechanisms, such as the extinction of pain-related fear. Instead, they solely focus on training generic coping strategies that help patients minimize the burden of the disease. Strategies include goal-setting, learning to communicate effectively with health care providers, and adopting a structured approach towards making important decisions. The interventions often include peer support activities and can even be provided by lay teachers (e.g. expert patients) with no background in health care. For patients with chronic pain, this approach can be important for at least two reasons. First, patients often report difficulties in self-management skills such as planning ahead and finding resources (Corcoran et al., 2010; Lennox Thompson et al., 2020). Second, self-management programmes completely focus on supporting patients in how to self-regulate their condition throughout their lives. This approach may therefore incorporate important principles on how to deal with relapse, which could be used to improve IMPT programmes.

\section{AIMS AND OUTLINE}

The first aim of this dissertation is to provide an overview of the current state of literature on the longitudinal outcomes and treatment characteristics of IMPT programmes. Although the effectiveness of IMPT programmes compared to control treatments at specific time points has been investigated in systematic reviews (e.g. Kamper et al., 2015), it is unclear to what extent any treatment gains are maintained over time. Chapter two contains the results of a systematic review that describes the patterns of change over time in patient cohorts participating in IMPT programmes. The study also includes a detailed overview of heterogeneity regarding study, patient, and intervention characteristics, using the Template for Intervention Description and Replication (TIDieR) checklist. In Chapter three, we propose a 'living workflow', an iterative yearly updating cycle, that is intended to keep the outcomes of the review in Chapter 2 up-to-date.

The second aim of this research project is to develop an intervention that facilitates the maintenance of treatment gains over time. Chapter four describes the results of a systematic review and meta-analysis on generic self-management interventions for 
patients with chronic pain. The results include an overview of the variability in dose and content between the programmes. The study adopts a GRADE approach towards determining the effectiveness of generic self-management interventions on outcomes related to daily life functioning and quality of life. To further accommodate the paucity of information on the prevention of relapse, we opted for a co-design approach. This method is characterized by increments in knowledge over multiple development cycles, and by the continuous involvement of stakeholders in the design process. In Chapter five, we reflect on the value and function of the co-design approach by presenting the intervention development project as a case study and by systematically reflecting on each design phase. Chapter six contains the first field test of the prototype that originated from this project. Based on these findings, we further developed the prototype into an mHealth application. In Chapter seven, we outline this process and present a study protocol for a randomized controlled trial to test its effectiveness. Chapter eight contains the general discussion. In this section, we will specifically evaluate the implications for both clinical practice and research and discuss the potential impact of our main outcomes. Box 1 includes all the questions that we address in this thesis. 
Box 1. Questions Within the Current Thesis

Aim 1: to provide an overview of the current state of literature regarding treatment characteristics and longitudinal outcomes of IMPT programmes.

Chapter 2

- How does the physical, psychological and social wellbeing of patients with chronic pain who participated in IMPT programmes change over time?

- To what extent do the included studies vary with respect to study design, patient and treatment characteristics?

Chapter 3

- How can we transition a regular review into a living systematic review to increase the speed of dissemination and implementation of scientific findings into clinical practice?

Aim 2: to develop an intervention that facilitates the maintenance of treatment gains and insights over time.

Chapter 4

- What are the immediate and long-term effects of generic self-management interventions for patients with chronic musculoskeletal pain in terms of physical functioning, self-efficacy, pain intensity and physical activity?

- Which programme characteristics, components, and behaviour change techniques are included in the selfmanagement interventions?

Chapter 5

- To what extent do co-design practices facilitate the translation of meaningful stakeholder experiences into the design of a health care intervention?

- How can co-design contribute to stakeholder involvement, generation of relevant insights and ideas, and incorporation of stakeholder input into the intervention design?

Chapter 6

- How do patients and health care providers evaluate the current workbook prototype in the context of IMPT programmes in terms of acceptability and applicability?

Chapter 7

- How can we evaluate the effectiveness of the AGRIPPA mHealth intervention, determine its cost-effectiveness and acquire insight into its level of engagement and usability? 


\section{REFERENCES}

Åkerblom, S., Perrin, S., Rivano Fischer, M., \& McCracken, L. M. (2015). The Mediating Role of Acceptance in Multidisciplinary Cognitive-Behavioral Therapy for Chronic Pain. J Pain, 16(7), 606-615. https://doi. org/10.1016/j.jpain.2015.03.007

Allegrante, J. P., Wells, M. T., \& Peterson, J. C. (2019). Interventions to Support Behavioral Self-Management of Chronic Diseases. Annu Rev Public Health, 40(1), 127-146. https://doi.org/10.1146/annurev-publhealth-040218-044008

Backs-Dermott, B. J., Dobson, K. S., \& Jones, S. L. (2010). An evaluation of an integrated model of relapse in depression. J Affect Disord, 124(1-2), 60-67. https://doi.org/10.1016/j.jad.2009.11.015

Basbaum, A. I., Bautista, D. M., Scherrer, G., \& Julius, D. (2009). Cellular and Molecular Mechanisms of Pain. Cell, 139(2), 267-284. https://doi.org/10.1016/j.cell.2009.09.028

Berryman, C., Stanton, T. R., Bowering, J. K., Tabor, A., McFarlane, A., \& Moseley, L. G. (2013). Evidence for working memory deficits in chronic pain: A systematic review and meta-analysis. Pain, 154(8), 1181-1196. https://doi. org/10.1016/j.pain.2013.03.002

Blyth, F. M., Briggs, A. M., Schneider, C. H., Hoy, D. G., \& March, L. M. (2019). The global burden of musculoskeletal pain-where to from here? Am J Public Health, 109(1), 35-40. https://doi.org/10.2105/AJPH.2018.304747

Boudreaux, M. J., \& Ozer, D. J. (2013). Goal conflict, goal striving, and psychological well-being. Motiv Emot, 37(3), 433-443. https://doi.org/10.1007/s11031-012-9333-2

Breivik, H., Collett, B., Ventafridda, V., Cohen, R., \& Gallacher, D. (2006). Survey of chronic pain in Europe: Prevalence, impact on daily life, and treatment. Eur J Pain, 10(4), 287-287. https://doi.org/10.1016/j.ejpain.2005.06.009

Brodal, P. (2017). A neurobiologist's attempt to understand persistent pain. Scand J Pain, 15(1), 140-147. https://doi. org/10.1016/j.sjpain.2017.03.001

Carver, C. S., \& Scheier, M. F. (2017). Self-Regulatory Functions Supporting Motivated Action. In Adv in Mot Sci, Vol. 4, pp. 1-37. Elsevier. https://doi.org/10.1016/bs.adms.2017.02.002

Chou, R., Deyo, R., Friedly, J., Skelly, A., Hashimoto, R., Weimer, M., Fu, R., Dana, T., Kraegel, P., Griffin, J., Grusing, S., \& Brodt, E. D. (2017). Nonpharmacologic Therapies for Low Back Pain: A Systematic Review for an American College of Physicians Clinical Practice Guideline. Ann Intern Med, 166(7), 493. https://doi.org/10.7326/M162459

Claes, N., Vlaeyen, J. W. S., Lauwerier, E., Meulders, M., \& Crombez, G. (2018). Goal conflict in chronic pain: day reconstruction method. PeerJ, 6(8), e5272. https://doi.org/10.7717/peerj.5272

Clauw, D. J., Essex, M. N., Pitman, V., \& Jones, K. D. (2019). Reframing chronic pain as a disease, not a symptom: rationale and implications for pain management. Postgrad Med, 131(3), 185-198. https://doi.org/10.1080/00 325481.2019.1574403

Corcoran, T. B., Haigh, F., Seabrook, A., \& Schug, S. A. (2010). A Survey of Patients' Use of the Internet for Chronic Pain-Related Information. Pain Med, 11(4), 512-517. https://doi.org/10.1111/j.1526-4637.2010.00817.x

Darlow, B., Dowell, A., Baxter, G. D., Mathieson, F., Perry, M., \& Dean, S. (2013). The Enduring Impact of What Clinicians Say to People With Low Back Pain. Ann Fam Med, 11(6), 527-534. https://doi.org/10.1370/afm.1518

Doleys, D. M. (2017). Chronic Pain as a Hypothetical Construct: A Practical and Philosophical Consideration. Front Psychol, 8, 1-7. https://doi.org/10.3389/fpsyg.2017.00664

Eccleston, C. (2018). Chronic pain as embodied defence: implications for current and future psychological treatments. Pain, 159(1), S17-S23. https://doi.org/10.1097/j.pain.0000000000001286

Eccleston, C., \& Crombez, G. (2007). Worry and chronic pain: A misdirected problem solving model. Pain, 132(3), 233-236. https://doi.org/10.1016/j.pain.2007.09.014 
Edwards, K. A., Pielech, M., Hickman, J., Ashworth, J., Sowden, G., \& Vowles, K. E. (2019). The relation of selfcompassion to functioning among adults with chronic pain. European J Pain, 23(8), 1538-1547. https://doi. org/10.1002/ejp.1429

Edwards, R. R., Dworkin, R. H., Sullivan, M. D., Turk, D. C., \& Wasan, A. D. (2016). The Role of Psychosocial Processes in the Development and Maintenance of Chronic Pain. J Pain, 17(9), T70-T92. https://doi.org/10.1016/j. jpain.2016.01.001

Elzahaf, R. A., Tashani, O. A., Unsworth, B. A., \& Johnson, M. I. (2012). The prevalence of chronic pain with an analysis of countries with a Human Development Index less than 0.9: a systematic review without meta-analysis. Curr Med Res Opin, 28(7), 1221-1229. https://doi.org/10.1185/03007995.2012.703132

Erdil, M., Bilsel, K., Imren, Y., Ceylan, H. H., \& Tuncay, I. (2012). Total hip arthroplasty in a patient with congenital insensitivity to pain: a case report. J Med Case Rep, 6(1), 190. https://doi.org/10.1186/1752-1947-6-190

Gatchel, R. J., McGeary, D. D., McGeary, C. A., \& Lippe, B. (2014). Interdisciplinary chronic pain management: Past, present, and future. Am Psychol, 69(2), 119-130. https://doi.org/10.1037/a0035514

Hasenbring, M. I., Andrews, N. E., \& Ebenbichler, G. (2020). Overactivity in Chronic Pain, the Role of Painrelated Endurance and Neuromuscular Activity. Clin J Pain, 36(3), 162-171. https://doi.org/10.1097/ AJP.0000000000000785

Höchli, B., Brügger, A., \& Messner, C. (2018). How Focusing on Superordinate Goals Motivates Broad, Long-Term Goal Pursuit: A Theoretical Perspective. Front Psychol, 9, 1-14. https://doi.org/10.3389/fpsyg.2018.01879

Hruschak, V., \& Cochran, G. (2017). Psychosocial and environmental factors in the prognosis of individuals with chronic pain and comorbid mental health. Soc Work Health Care, 56(7), 573-587. https://doi.org/10.1080/009 81389.2017.1326074

IASP. (2021, March 22). IASP Terminology - IASP. IASP. https://www.iasp-pain.org/terminology?navItemNumber=576

IASP. (2021, March 22). In Memoriam: John J. Bonica - IASP. IASP. https://www.iasp-pain.org/AboutIASP/Content. aspx?ItemNumber $=1129$

Jensen, M. P., Moore, M. R., Bockow, T. B., Ehde, D. M., \& Engel, J. M. (2011). Psychosocial Factors and Adjustment to Chronic Pain in Persons With Physical Disabilities: A Systematic Review. Arch Phys Med Rehabil, 92(1), 146-160. https://doi.org/10.1016/j.apmr.2010.09.021

Kaiser, U., Kopkow, C., Deckert, S., Neustadt, K., Jacobi, L., Cameron, P., De Angelis, V., Apfelbacher, C., Arnold, B., Birch, J., Bjarnegård, A., Christiansen, S., C de C Williams, A., Gossrau, G., Heinks, A., Hüppe, M., Kiers, H., Kleinert, U., Martelletti, P., ... Schmitt, J. (2018). Developing a core outcome domain set to assessing effectiveness of interdisciplinary multimodal pain therapy: the VAPAIN consensus statement on core outcome domains. Pain, 159(4), 673-683. https://doi.org/10.1097/j.pain.0000000000001129

Kaiser, U., Treede, R.-D., \& Sabatowski, R. (2017). Multimodal pain therapy in chronic noncancer pain-gold standard or need for further clarification? Pain, 158(10), 1853-1859. https://doi.org/10.1097/j.pain.0000000000000902

Kamper S. J., Apeldoorn A. T., Chiarotto A., Smeets R. J., Ostelo R. W. J. G., Guzman J., van Tulder M. W. (2014). Multidisciplinary biopsychosocial rehabilitation for chronic low back pain. Cochrane Database of Syst Rev, 9. https://doi.org/10.1002/14651858.CD000963.pub3.

Karoly, P. (2020). How Pain Shapes Depression and Anxiety: A Hybrid Self-regulatory/Predictive Mind Perspective. J Clin Psychol Med Settings. https://doi.org/10.1007/s10880-019-09693-5

Kosek, E., Cohen, M., Baron, R., Gebhart, G. F., Mico, J.-A., Rice, A. S. C., Rief, W., \& Sluka, A. K. (2016). Do we need a third mechanistic descriptor for chronic pain states? Pain, 157(7), 1382-1386. https://doi.org/10.1097/j. pain.0000000000000507

Lennox Thompson, B., Gage, J., \& Kirk, R. (2020). Living well with chronic pain: a classical grounded theory. Disabil and Rehabil, 42(8), 1141-1152. https://doi.org/10.1080/09638288.2018.1517195 
Lorig, K. R., \& Holman, H. R. (2003). Self-management education: History, definition, outcomes, and mechanisms. Ann of Behav Med, 26(1), 1-7. https://doi.org/10.1207/S15324796ABM2601_01

Martins, C., Dutton, G. R., Hunter, G. R., \& Gower, B. A. (2020). Revisiting the Compensatory Theory as an explanatory model for relapse in obesity management. Am J Clin Nutr, 112(5), 1170-1179. https://doi.org/10.1093/ajcn/ nqaa243

McCarberg, B., \& Peppin, J. (2019). Pain Pathways and Nervous System Plasticity: Learning and Memory in Pain. Pain Med, 20(12), 2421-2437. https://doi.org/10.1093/pm/pnz017

McCarthy, C. J., \& Yeowell, G. (2017). Newspaper response to the back pain myth busting advice: bruising but helpful. Br J Sports Med, 51(9), 758-758. https://doi.org/10.1136/bjsports-2016-097253

McCracken, L. M., \& Vowles, K. E. (2014). Acceptance and commitment therapy and mindfulness for chronic pain: Model, process, and progress. Am Psychol, 69(2), 178-187. https://doi.org/10.1037/a0035623

Mescouto, K., Olson, R. E., Hodges, P. W., \& Setchell, J. (2020). A critical review of the biopsychosocial model of low back pain care: time for a new approach? Disabil Rehabil, 5, 1-15. https://doi.org/10.1080/09638288.2020.18 51783

Moore, D. J., Meints, S. M., Lazaridou, A., Johnson, D., Franceschelli, O., Cornelius, M., Schreiber, K., \& Edwards, R. R. (2019). The Effect of Induced and Chronic Pain on Attention. J Pain, 20(11), 1353-1361. https://doi. org/10.1016/j.jpain.2019.05.004

Moriarty, O., McGuire, B. E., \& Finn, D. P. (2011). The effect of pain on cognitive function: A review of clinical and preclinical research. Prog Neurobiol 93(3), 385-404. https://doi.org/10.1016/j.pneurobio.2011.01.002

Morley, S. (2008). Relapse prevention: Still neglected after all these years. Pain, 134(3), 239-240. https://doi. org/10.1016/j.pain.2007.12.004

Morley, S., Williams, A., \& Eccleston, C. (2013). Examining the evidence about psychological treatments for chronic pain: Time for a paradigm shift? Pain, 154(10), 1929-1931. https://doi.org/10.1016/j.pain.2013.05.049

Moseley, G. L., \& Vlaeyen, J. W. S. (2015). Beyond nociception. Pain, 156(1), 35-38. https://doi.org/10.1016/j. pain.0000000000000014

Nijs, J., Roussel, N., Paul van Wilgen, C., Köke, A., \& Smeets, R. (2013). Thinking beyond muscles and joints: Therapists' and patients' attitudes and beliefs regarding chronic musculoskeletal pain are key to applying effective treatment. Man Ther, 18(2), 96-102. https://doi.org/10.1016/j.math.2012.11.001

O’Sullivan, P. B., Caneiro, J. P., O’Keeffe, M., Smith, A., Dankaerts, W., Fersum, K., \& O’Sullivan, K. (2018). Cognitive Functional Therapy: An Integrated Behavioral Approach for the Targeted Management of Disabling Low Back Pain. Phys Ther, 98(5), 408-423. https://doi.org/10.1093/ptj/pzy022

Phelps, C. E., Navratilova, E., \& Porreca, F. (2021). Cognition in the Chronic Pain Experience: Preclinical Insights. Trends Cog Sci, 25(5), 365-376. https://doi.org/10.1016/j.tics.2021.01.001

Pitcher, M. H., Von Korff, M., Bushnell, M. C., \& Porter, L. (2019). Prevalence and Profile of High-Impact Chronic Pain in the United States. J Pain, 20(2), 146-160. https://doi.org/10.1016/j.jpain.2018.07.006

Ramírez-Maestre, C., Esteve, R., López-Martínez, A. E., Serrano-Ibáñez, E. R., Ruiz-Párraga, G. T., \& Peters, M. (2019). Goal Adjustment and Well-Being: The Role of Optimism in Patients with Chronic Pain. Ann Behav Med, 53(7), 597-607. https://doi.org/10.1093/abm/kay070

Ramnerö, J., \& Törneke, N. (2015). On Having a Goal: Goals as Representations or Behavior. Psychol Rec, 65(1), 89-99. https://doi.org/10.1007/s40732-014-0093-0

Sanders, S. H. (2006). Behavioral conceptualization and treatment for chronic pain. The Behav Anal Today, 7(2), 253261. https://doi.org/10.1037/h0100082

Seymour, B. (2019). Pain: A Precision Signal for Reinforcement Learning and Control. Neuron, 101(6), $1029-1041$. https://doi.org/10.1016/j.neuron.2019.01.055 
Tabor, A., \& Burr, C. (2019). Bayesian Learning Models of Pain: A Call to Action. Curr Opin Behav Sci, 26, 54-61. https://doi.org/10.1016/j.cobeha.2018.10.006

Thacker, M. A., \& Moseley, G. L. (2012). First-person neuroscience and the understanding of pain. Med J Aus, 196(6), 410-411. https://doi.org/10.5694/mja12.10468

Todd, J., van Ryckeghem, D. M. L., Sharpe, L., \& Crombez, G. (2018). Attentional bias to pain-related information: a meta-analysis of dot-probe studies. Health Psychol Rev, 12(4), 419-436. https://doi.org/10.1080/17437199.201 8.1521729

Toye, F., Seers, K., Hannink, E., \& Barker, K. (2017). A mega-ethnography of eleven qualitative evidence syntheses exploring the experience of living with chronic non-malignant pain. BMC Med Res Methodol, 17(1), 116. https:// doi.org/10.1186/s12874-017-0392-7

Treede, R. D., Rief, W., Barke, A., Aziz, Q., Bennett, M. I., Benoliel, R., Cohen, M., Evers, S., Finnerup, N. B., First, M. B., Giamberardino, M. A., Kaasa, S., Kosek, E., Lavand'homme, P., Nicholas, M., Perrot, S., Scholz, J., Schug, S., Smith, B. H., ... Wang, S. J. (2015). A classification of chronic pain for ICD-11. Pain, 156(6), 1003-1007. https:// doi.org/10.1097/j.pain.0000000000000160

Treede, R.-D., Rief, W., Barke, A., Aziz, Q., Bennett, M. I., Benoliel, R., Cohen, M., Evers, S., Finnerup, N. B., First, M. B., Giamberardino, M. A., Kaasa, S., Korwisi, B., Kosek, E., Lavand'homme, P., Nicholas, M., Perrot, S., Scholz, J., Schug, S., ... Wang, S.-J. (2019). Chronic pain as a symptom or a disease: the IASP Classification of Chronic Pain for the International Classification of Diseases (ICD-11). Pain, 160(1), 19-27. https://doi.org/10.1097/j. pain.0000000000001384

Turk, D. C., \& Rudy, T. E. (1991). Neglected topics in the treatment of chronic pain patients - relapse, noncompliance, and adherence enhancement. Pain, 44(1), 5-28. https://doi.org/10.1016/0304-3959(91)90142-K

Vlaeyen, J. W. S., Crombez, G., \& Linton, S. J. (2016). The fear-avoidance model of pain. Pain, 157(8), 1588-1589. https://doi.org/10.1097/j.pain.0000000000000574

Vlaeyen, J. W. S., Morley, S., \& Crombez, G. (2016). The experimental analysis of the interruptive, interfering, and identity-distorting effects of chronic pain. Behav Res Ther, 86, 23-34. https://doi.org/10.1016/j.brat.2016.08.016

Vos, T., Abajobir, A. A., Abate, K. H., Abbafati, C., Abbas, K. M., Abd-Allah, F., Abdulkader, R. S., Abdulle, A. M., Abebo, T. A., Abera, S. F., Aboyans, V., Abu-Raddad, L. J., Ackerman, I. N., Adamu, A. A., Adetokunboh, O., Afarideh, M., Afshin, A., Agarwal, S. K., Aggarwal, R., ... Murray, C. J. L. (2017). Global, regional, and national incidence, prevalence, and years lived with disability for 328 diseases and injuries for 195 countries, 1990-2016: a systematic analysis for the Global Burden of Disease Study 2016. Lancet, 390(10100), 1211-1259. https://doi. org/10.1016/S0140-6736(17)32154-2

Waterschoot, F. P. C., Dijkstra, P. U., Hollak, N., de Vries, H. J., Geertzen, J. H. B., \& Reneman, M. F. (2014). Dose or content? Effectiveness of pain rehabilitation programs for patients with chronic low back pain: A systematic review. Pain, 155(1), 179-189. https://doi.org/10.1016/j.pain.2013.10.006

Weisman, A., Quintner, J., \& Masharawi, Y. (2019). Congenital Insensitivity to Pain: A Misnomer. J Pain, 20(9), 10111014. https://doi.org/10.1016/j.jpain.2019.01.331

Williams, A. C. de C., Fisher, E., Hearn, L., \& Eccleston, C. (2020). Psychological therapies for the management of chronic pain (excluding headache) in adults. Cochrane Database of Syst Rev, 2020(8). https://doi.org/10.1002/14651858. CD007407.pub4

Williams, A. C. de C. (2016). What can evolutionary theory tell us about chronic pain? Pain, 157(4), 788-790. https:// doi.org/10.1097/j.pain.0000000000000464

Woller, S. A., Eddinger, K. A., Corr, M., \& Yaksh, T. L. (2017). An overview of pathways encoding nociception. Clin Exp Rheumatol, 35(5, Suppl 107), 40-46. http://www.ncbi.nlm.nih.gov/pubmed/28967373

Wood, W., \& Neal, D. T. (2016). Healthy through habit: Interventions for initiating \& maintaining health behavior change. Behav Sci Pol, 2(1), 71-83. https://doi.org/10.1353/bsp.2016.0008 
Yozu, A., Haga, N., Funato, T., Owaki, D., Chiba, R., \& Ota, J. (2016). Hereditary sensory and autonomic neuropathy types 4 and 5: Review and proposal of a new rehabilitation method. Neurosci Res, 104, 105-111. https://doi. org/10.1016/j.neures.2015.10.011 



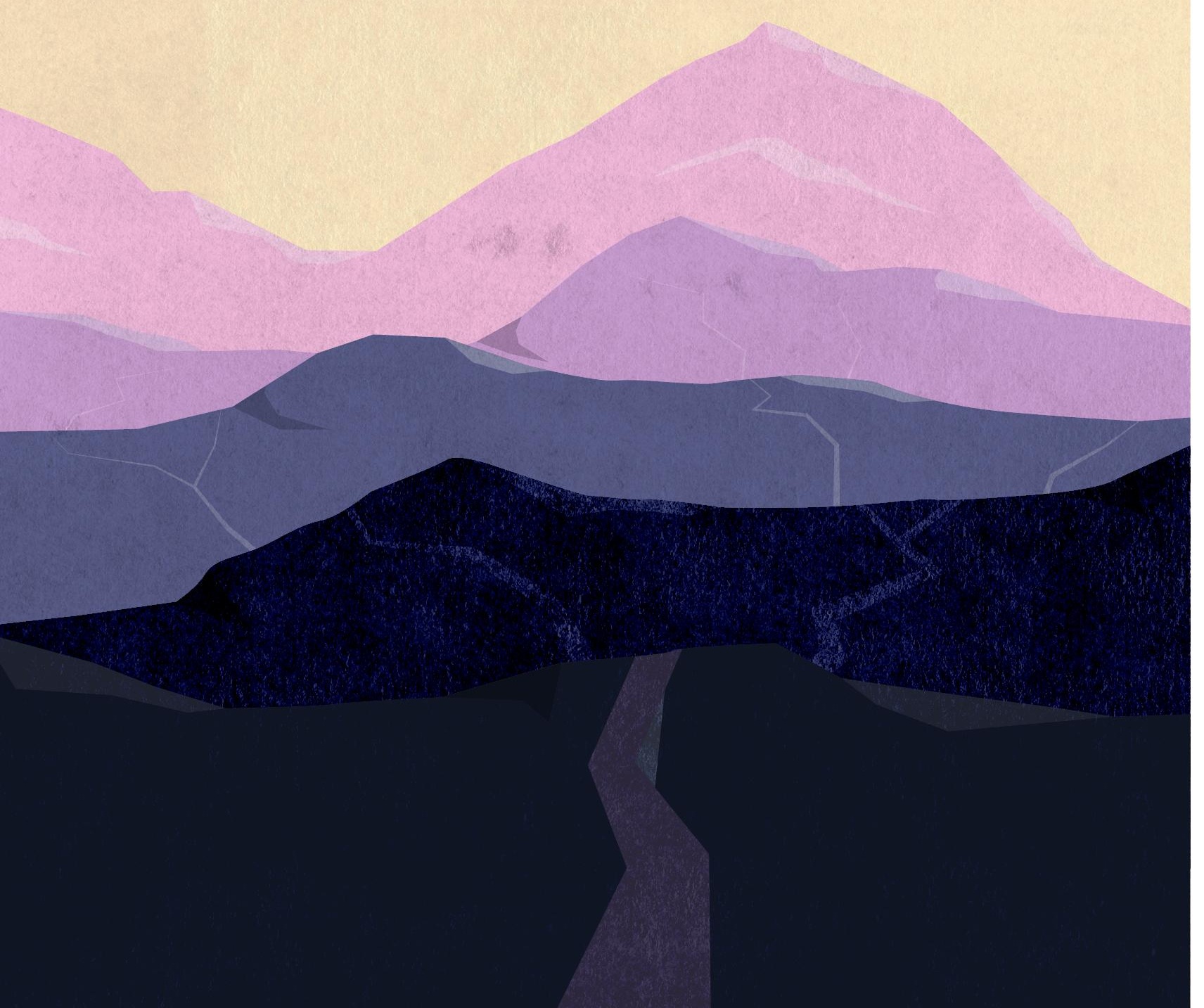




\title{
02
}

\section{Longitudinal Outcome Evaluations of Interdisciplinary Multimodal Pain Treatment Programmes for Patients With Chronic Primary Pain:}

\section{A Systematic Review and Meta-Analysis}

\author{
S. Elbers ${ }^{1,2}$, H. Wittink ${ }^{1 *}$, S. Konings ${ }^{3 *}$, U. Kaiser ${ }^{4,5}$, J. Kleijnen ${ }^{6}$, J. Pool ${ }^{1}$, A. Köke ${ }^{2,7,8}$, \\ R. Smeets ${ }^{2,9}$
}

Accepted in European Journal of Pain

1. Research group Lifestyle \& Health, Research Centre Healthy and Sustainable Living, University of Applied Sciences Utrecht, The Netherlands.

2. Department of Rehabilitation Medicine, Research School CAPHRI, Faculty of Health, Life Sciences and Medicine, Maastricht University, The Netherlands.

3. Department of Health Innovation and Technology, Fontys University of Applied Sciences, Eindhoven, The Netherlands.

4. Comprehensive Pain Center, Medical Faculty Technical University Dresden, Germany.

5. University Hospital Carl Gustav Carus Dresden, Germany.

6. Department of Family Medicine, Research School CAPHRI, Faculty of Health, Life Sciences and Medicine, Maastricht University, The Netherlands.

7. Centre of Expertise in Pain and Rehabilitation, Adelante, Hoensbroek, The Netherlands.

8. South University of Applied Sciences Heerlen, The Netherlands.

9. CIR Revalidatie, location Eindhoven, The Netherlands.

* These authors contributed equally. 


\section{ABSTRACT}

Background and Objectives: Although Interdisciplinary Multimodal Pain Treatment (IMPT) programmes share a biopsychosocial approach to increase the wellbeing of patients with chronic pain, substantial variation in content and duration have been reported. In addition, it is unclear to what extent any favorable health outcomes are maintained over time. Therefore, our first aim was to identify and analyse the change over time of patient related outcome measures in cohorts of patients who participated in IMPT programmes. Our second aim was to acquire insight into the heterogeneity of IMPT programmes. Databases and Data Treatment: The study protocol was registered in Prospero under CRD42018076093. We searched Medline, Embase, PsycInfo and Cinahl from inception to May 2020. All study selection, data extraction and risk of bias assessments were independently performed by two researchers. Study cohorts were eligible if they included adult patients with chronic primary pain for at least 3 months that was primarily perceived in musculoskeletal structures. We assessed the change over time, by calculating pre-post, post-follow-up and pre-follow-up contrasts for seven different patient-reported outcome domains. To explore the variability between the IMPT programmes, we summarized the patient characteristics and treatment programmes using the intervention description and replication checklist. Results: The majority of the 74 included patient cohorts significantly improved during treatment. Importantly, this improvement was generally maintained at follow-up. In line with our expectations and with previous studies, we observed substantial methodological and statistical heterogeneity. Conclusions: This study shows that participation in an IMPT programme is associated with considerable improvements in wellbeing that are generally maintained at follow-up. The current study also found substantial heterogeneity in dose and treatment content, which suggests different viewpoints on how to optimally design an IMPT programme.

Significance: The current study provides insight into the different existing approaches regarding the dose and content of IMPT programmes. This analysis contributes to an increased understanding of the various approaches by which a biopsychosocial perspective on chronic pain can be translated to treatment programmes. Furthermore, despite theoretical and empirical assertions regarding the difficulty to maintain newly learned health behaviours over time, the longitudinal analysis of health outcomes did not find a relapse pattern for patients who participated in IMPT programmes.

Keywords: Systematic review, Meta-analysis, Interdisciplinary, Chronic pain, Longitudinal, Rehabilitation. 


\section{INTRODUCTION}

Interdisciplinary multimodal pain treatment (IMPT) programmes are recognized as treatment of choice for patients with chronic pain (Gatchel et al., 2014; Turk, 2003). Since the 1970s these programmes have evolved towards interventions that combine (cognitive) behavioural approaches with exercise, medical treatment and education based on a biopsychosocial model. The aim of these programmes is not to target pain itself, but to help patients to optimize daily life functioning and to increase social, physical and psychological wellbeing (Gatzounis et al., 2012; Kaiser et al., 2017; Penney \& Haro, 2019). This approach is typically provided by rehabilitation centers or hospitals and requires the expertise of an interdisciplinary team of healthcare providers. Generally, these disciplines cover the biopsychosocial spectrum and continuously coordinate their treatment activities and align them to patient-specific goals.

Despite common historical roots and a biopsychosocial perspective on chronic pain, substantial variation in content, duration and outcome evaluations of IMPT programmes has been reported. For example, systematic reviews found that the total treatment duration varied between 6.4 and 196.8 hours, programmes were delivered in both inpatient and outpatient settings, and pain-related disability was measured with 12 different measurement instruments (Kamper et al., 2014; Scascighini et al., 2008; Waterschoot et al., 2014). This variability not only hinders a meaningful interpretation of pooled effect sizes, it also reflects uncertainty regarding optimal dose, content and the selection of measurement instruments (Waterschoot et al., 2014).

A second problem regarding the current evidence-base of IMPT programmes is that it is unclear to what extent treatment gains are maintained over time. Although RCTs often indicate a statistically significant effect compared to control interventions, post treatment assessments still suggest a considerable impact on daily life functioning (Kamper et al., 2014). From a clinical perspective, this indicates that most patients continue to experience the burdening effect of pain after treatment. This may be problematic, as the newly learned pain management strategies are considered to be fragile and vulnerable to disruptions (e.g. unexpected exacerbations of symptoms, an unforeseen event in the personal context or nocebo's). Continuing occurrences of pain interference could prompt pre-treatment coping strategies, resulting in a declined effect over time (Carver \& Scheier, 2017; Vlaeyen et al., 2016). Although this so-called 'triangular relapse pattern' - with an improvement from pre-intervention to post-intervention, followed by an unfavorable trend at follow-up - has been observed in other healthcare domains, this topic has been neglected in the field of pain rehabilitation (Brouwer et al., 2019; Morley, 2008; Opozda et al., 2016; Turk \& Rudy, 
1991; Wilson, 2010; Wood \& Neal, 2016). To understand the impact of these programmes on patients' ability to self-regulate their wellbeing after completion of the treatment programme, it is crucial to assess the change of IMPT outcomes over time.

To acquire insight in both evidence-gaps, our first aim was to identify and analyse the change over time of key outcome measures in patients with chronic pain who participated in IMPT programmes. Therefore, the first research question is: How does the physical, psychological and social wellbeing of patients with chronic musculoskeletal pain who participated in IMPT programmes change over time? Our second aim was to explore the heterogeneity of study, patient, intervention and outcome characteristics: To what extent do cohorts vary with respect to study, patient and treatment characteristics?

\section{LITERATURE SEARCH METHODS}

\section{Protocol and Registration}

The study was reported in line with the PRISMA guidelines (Moher et al., 2009; Moher et al., 2015) and the study protocol has been registered in PROSPERO under CRD42018076093.

\section{Search}

We performed our search in Medline and Embase via OVID, and PsycInfo and Cinahl via EBSCOhost from inception to May 2020. The search string was developed by experienced reviewers (SE and JK) and consisted of multiple blocks that were combined with Boolean operators (see Supplementary file 1). Each block included free text words as well as specific subject headings. In addition, we searched for grey literature including unpublished studies in the Dart Europe, Open access Theses and Dissertations, NDLTD, ClinicalTrials.gov and WHO ICTRP databases. For each included study we also performed forward (in Google Scholar) and backward reference searches.

\section{Eligibility Criteria}

Randomized controlled trials, as well as case series and cohort studies were included. Study cohorts had to include adult patients with chronic primary pain for at least 3 months that was primarily perceived in musculoskeletal structures (e.g. bones, joints, muscles or related soft tissues) (Treede et al., 2015). In case of mixed cohorts, at least $75 \%$ of the patients had to experience musculoskeletal pain. The criteria for IMPT programmes were based on the definition of Gatchel and colleagues and had to include (a) a common philosophy treatment in line with the biopsychosocial model of pain; (b) a treatment component where patients actively participated by means of tasks, training and/or exercise; (c) at least three different 
healthcare professionals from various disciplines that provided the interdisciplinary treatment; (d) a single facility where each patient received treatment (Gatchel et al., 2014). This last criterion excluded care-network settings, but not multicenter trials. Although structured team meetings are considered an important aspect of IMPT programmes (Kaiser et al., 2017), we did not include this as an inclusion criterion, because we expected that not all studies would explicitly report this. Our outcomes were based on the criteria developed by the Initiative on Methods, Measurement, and Pain Assessment in Clinical Trials (IMMPACT) and included physical functioning, pain interference, depression, anxiety, emotional functioning, anger, self-efficacy, social functioning and pain intensity (see protocol for rationale) (Dworkin et al., 2005; Turk et al., 2003). The study had to include at least one outcome that was measured at two time points: prior to treatment and at least 12 months after the intervention was completed. Studies that focused on patients with postsurgical pain or cancer pain, as well as studies that solely included patients on the basis of a specific comorbidity (e.g. depression) were excluded. Articles published in other languages than English, German or Dutch were also excluded.

\section{Study Selection, Data Extraction and Risk of Bias}

All study selection, data extraction and risk of bias assessments were independently performed by at minimum two different researchers (UK and SE for articles in German, SK, SE and MK for articles in other languages). Researchers used pre-tested forms and compared their input to reach consensus. In case of disagreement, the study was discussed with other researchers (HW and RS) for a final decision. Study selection was performed in two rounds. In the screening round, abstracts were screened using the Rayyan software package (Ouzzani et al., 2016). Subsequently, full text studies were assessed on all eligibility criteria.

From the extraction round onwards, we considered patient cohorts - not journal articles - as our primary unit of analysis. In case of multiple articles describing the same cohort, we combined these sources to construct a complete overview of the development over time. The first published article that met our eligibility criteria was used as the primary source and we consulted additional sources, such as protocols or follow-up studies if they contained additional relevant information. If the information sources did not contain all data items of interest, we did not contact the study authors but coded this as 'not reported' in our dataset. Our data extraction form included all items from the template for intervention description and replication (TIDieR) checklist to describe the content of the treatment programme in detail (supplementary 2) (Hoffmann et al., 2014). Risk of Bias was assessed with the Joanna Briggs Institute Checklist for Case Series, which included 10 criteria (Moola et al., 2017). A response of 'no' to any one of the items resulted in a high risk 
of bias, unless we found a clear indication of a limited impact of that item on the overall study outcome. The risk of bias form, including the scoring instructions, are available in the online multimedia appendix.

\section{Data Analysis}

The data extraction form included sample size (per measurement moment), age, sex, pain duration, nationality, method of recruitment, patient eligibility criteria, exclusion criteria, study design, type of outcome measures, and outcomes for all available time points on measurement instruments of interest. If treatment intensity was expressed in days, we assumed 6 hours of treatment per day. Because IMPT programmes are generally considered as a treatment of last resort, we specifically paid attention to obtaining information on attrition (Jeffery et al., 2011). We obtained pre, post and final follow-up sample sizes to calculate attrition rates for post-treatment and follow-up. When a cohort presented data for two or more outcome measures within one domain, we selected the most commonly used instrument.

Descriptive Analysis. To investigate the heterogeneity between the included IMPT programmes, study, patient and intervention characteristics were summarized in tables. Intervention descriptions were extracted and each separate component was then classified into one of 10 possible categories. Education referred to modalities that were primarily concerned with transfer of information from healthcare providers or experts to patients. All modalities regarding physical training, such as stretching, hydrotherapy and walking were categorized as exercise. Graded activity was only coded if the modality explicitly used the term graded activity or if the activities gradually and time-contingent increased after a baseline measurement. Modalities that described (cognitive) behavioural approaches, including problem solving training, exposure in vivo, rational emotive therapy or ACT were classified as (cognitive) behavioural treatment. Breathing techniques, autogenic training, mindfulness, and applied relaxation techniques were classified as relaxation. Modalities that mainly focused on training general coping skills and self-regulation were categorized as self-management skills. This included, goal setting activities, general pacing techniques, structuring of daily activities and ergonomics. Pharmacological treatment was only coded when medication was provided in response to chronic pain. Medication withdrawal procedures were coded as 'other'. Workplace visits, and ergonomic advice at the workplace were coded as workplace advice. The category body awareness included physical awareness and psychomotor exercises that aimed to improve the recognition of bodily signals. The last category - team meetings - was only coded when the patient actively participated in the team meetings. The categories were inductively developed by first extracting and then clustering the modalities of the first search into global categories (by SE and SK). In the final dataset, these 10 categories covered more than $90 \%$ of the treatment modalities. 
All remaining modalities were coded as 'other'. The description of each of the modalities and the classification were registered. A similar process was performed for healthcare providers. The following professions were coded as 'physician': occupational physician, rehabilitation physician, general practitioner and not otherwise specified physician. Other physician specialists (e.g. psychiatrist, orthopedic surgeon, anesthesiologist) who were mainly involved in a consulting instead of a coordinating role were coded as 'other'. Disciplines such as clinical psychologists, general psychologists and behavioural therapists were classified as 'psychologist'. Physical therapists and physiotherapists were classified as 'physical therapist'. Social workers and social counselors were classified as 'social worker'. Occupational therapists and nurses were classified accordingly.

To assess to what extent treatment programmes aligned their programme with individual patient characteristics and preferences (i.e. tailoring), we classified each programme into low, medium or high tailoring. We defined low tailoring as any form of personalized goalsetting, because this would allow patients to relate treatment content and progress to their personal situation. All studies received at minimum a 'low' tailoring classification because we assumed that all interdisciplinary programmes include some form of collaborative goalsetting at the start of treatment. We classified programmes as medium tailoring, when they selected or optionally provided specific treatment components based on patient-specific needs or preferences. High tailoring involved a fully personalized treatment programme, with varying duration and treatment activities and modules, based on each patient's clinical assessment.

Main data analysis. In addition to pain intensity, we included seven key outcome measures as outcomes in this analysis, divided over three domains: physical health, mental health and social health. For physical health, we included physical functioning and pain interference. We extracted of the outcomes depression, anxiety, anger, and self-efficacy beliefs within the mental health domain. For social health we only included social functioning. All outcomes were defined in the study protocol. For each of these outcomes that were present within a cohort, we used the available data to calculate effect sizes for pre-post, post-follow-up and pre-follow-up contrasts. To calculate effect sizes, we used the method of Becker et al's standardized mean change (SMC) (1988), with the modifications that were suggested by Morris (2000). The model assumes that the outcomes are normally distributed at both time points, with separate means but equal variances. Furthermore, the model corrects for a pre-post within-group correlation. Because we did not have access to the original data of the included cohorts, we imputed this value (Lipsey \& Wilson, 2001). For all studies, we imputed the median correlation $(r=.59)$ of a meta-analysis that investigated the range of within-group correlation values in active treatment groups (Balk et al., 2012). This value is comparable to other studies that have imputed within-group meta-analyses (Clond, 2016; 
Roberts et al., 2017). In addition, a sensitivity analysis for the within-subject correlation is available in the multimedia appendix for all r-values between 0 and 1 . Sample sizes lower than $n=10$ were not included in the analysis, because this could lead to inaccurate estimates of the standardized mean gain (Morris 2000). In some interventions, the main treatment programme was followed by follow-up treatment activities to enhance maintenance. In these situations, we considered end of treatment as the moment that the main treatment programme (i.e.. that covered the core of the treatment procedures) ended. Hence, followup meetings, booster sessions or reinforcement sessions were not considered as main treatment and could continue after post-treatment assessments. All assessments within 1 month after end of treatment were considered as a 'post' measure. We used the last available time-point for the follow-up contrast. We calculated standard deviations from standard errors by multiplying them with the square root of the corresponding sample size (Higgins et al., 2019). If medians and range were provided, we used the formula of Hozo and colleagues (2005) to estimate the mean value and corresponding SD. For studies that presented change scores, we calculated final value mean scores and imputed the baseline standard deviation. If the latter was not available, the study was not included in the metaanalysis. For medians and inter quartile ranges, we estimated means and SDs using the assumption that the interquartile range (IQR) width is 1.35 SD (Higgins et al., 2019). In case of missing measures of variability at follow-up, we imputed the baseline value or otherwise used the mean SD of the remaining trials that reported on that outcome. If data of the cohort was presented for different subgroups, we calculated one composite mean and SD. For data that was only presented in figures (e.g. boxplots) we measured the central tendency and measure of dispersion if the figure was of sufficient quality.

Subsequently, we summarized the effect sizes per outcome, by describing the direction of effect for each of the included cohorts over time. We a priori decided not to perform any pooling, because this was not in line with our study aims and we expected substantial heterogeneity among the included studies. To facilitate interpretation of the effect sizes, we re-expressed the median pre-post effect size on the most commonly used measurement instrument, using the weighted standard deviation of all available post-intervention scores of that instrument. To assess the statistical heterogeneity of the study outcomes we also calculated the $I^{2}$ and the $Q$ test for each outcome domain at every time point. A statistically significant $Q$ test rejects the hypothesis that all effect sizes are equal (Huedo-Medina et al., 2006). In addition, the $I^{2}$ index provides an indication of the proportion of variability in observed effects that is either due to between-study variability or due to within-study variability (i.e. sampling error) (Borenstein et al., 2017). This analysis was performed with the R metaphor package in RStudio (R Core Team, 2013; RStudio Team, 2020; Viechtbauer, 2010). 
Exploratory data analysis. To further explore the included cohorts, we developed an online multimedia appendix that contains interactive forest plots for each outcome, time-series and study characteristic tables. The appendix can be accessed via https:// datascience.hu.nl/rsconnect/impt-cohorts/. The time-series show the development over time of a measurement on a standardized scale (expressed as a percentage of the maximum score of each particular measurement instrument), as well as the raw scores and standard deviations. To standardize the scores, we obtained the distance of each mean and the unfavorable end of the scale, divided by the total distance of the scale and multiplied by 100 . These plots also allow for the comparison of specific cohorts over time with respect to a particular outcome. To accommodate future updates of this systematic review, the appendix also contains contact information to encourage readers to pinpoint any inaccuracies or to suggest cohorts that have not yet been included in the current review. Lastly, all data extraction files are listed in this appendix, including any comments that have been made regarding handling specific difficulties with that specific cohort (e.g. dealing with change scores, or imputing missing SDs). Supplementary file 3 contains the $\mathrm{R}$ code that has been used for all analyses in this review as well as the deployment of the appendix. The most recent version can also be accessed in a Github repository via: https:// github.com/stefanelbers/impt.meta_analysis/tree/master/deployment.

\section{RESULTS}

\section{Results of the Search}

The initial search was performed in May 2019 and was updated in May 2020 using the last date of the original search as the beginning date for the update. In total, the search yielded 31933 hits. After deduplication, 17988 studies remained. the screening of title and abstracts yielded 380 hits. In the final selection round, we obtained the full text versions and included 65 studies. 315 studies were excluded: 50 studies due to study design or publication type, 42 studies related to patient criteria, 89 studies due to intervention criteria, 89 studies because they did not include outcomes within the scope of this study or did not include a follow-up measurement of 12 months or longer, 38 studies were duplicates and 7 studies due to language or inclusion of patients with specific comorbidities. Seven of the included studies did not provide the necessary data (i.e. central tendency and measure of dispersion for each time point) to be included the quantitative analyses and were only included in the characteristics tables. The study flow is depicted in Figure 1. 


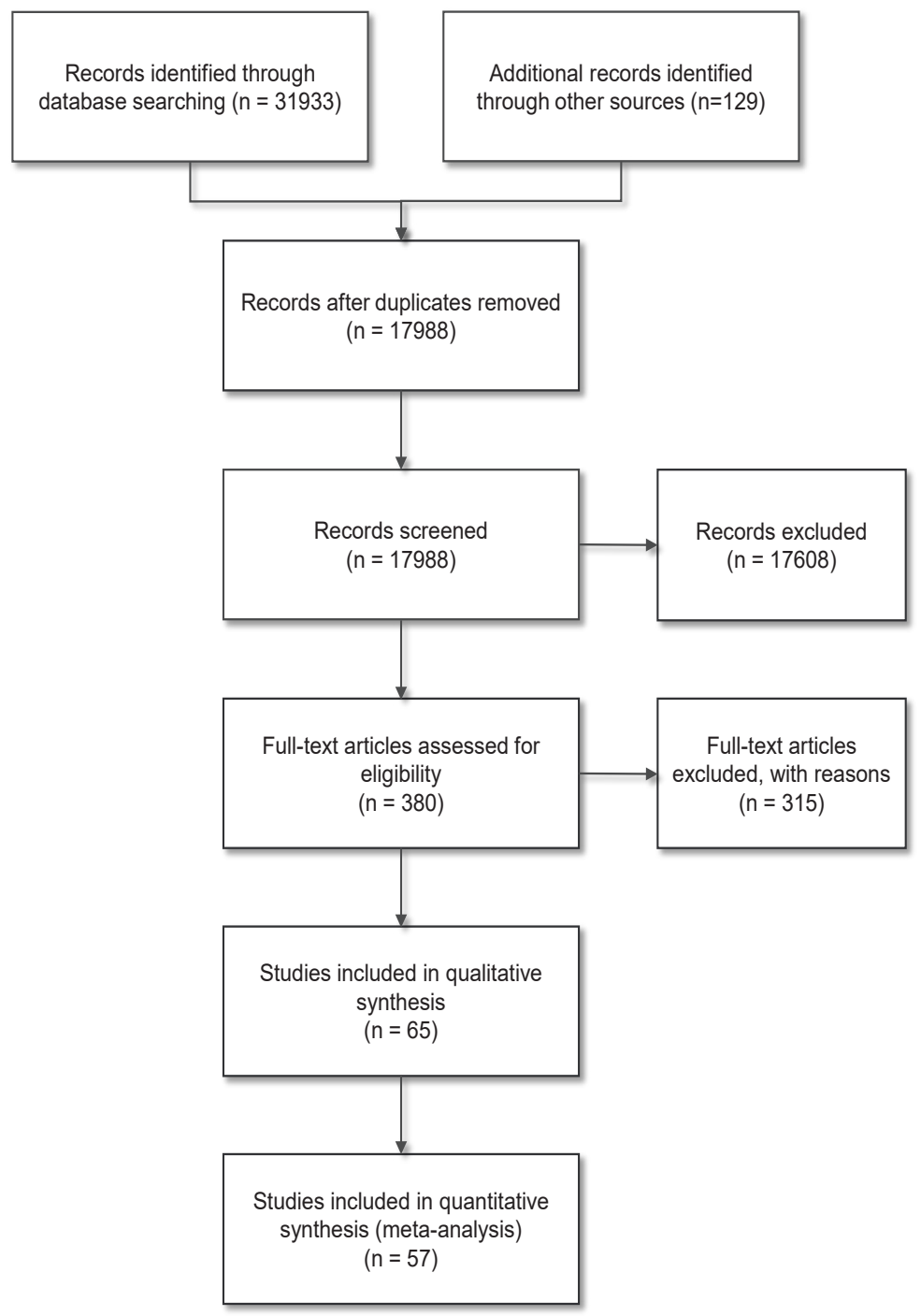

Figure 1. Flow Chart of Study Records. 


\section{Characteristics of the Included Studies}

Study Characteristics. We included 37 case series, (i.e. longitudinal studies with prospectively collected data on one cohort of patients), 19 RCTs, 4 N-RCTs and 5 other types of study design. In total, these studies provided data on 74 cohorts with a median final follow-up measurement of 12 months (range 12 - 120). Pretreatment sample sizes of the cohorts ranged from 10 to 2089, with a median of 102 . After filtering out cohorts that reported no dropout in the study flow (assuming a complete case analysis) the median dropout ratio for the 53 remaining cohorts was 8.56 (range $0-42.11$ ) at post treatment and 16.67 (range $=-3.85-62.17$ ) at final follow-up, using the posttreatment sample size as reference. Six of the 65 studies were evaluated as low risk of bias, indicating that in the majority of the cohorts we identified at least one factor that threatened the internal validity. Statistical analysis and attrition (67.69\%), unclear inclusion criteria (41.54\%), incomplete inclusion of participants (61.54\%), and incomplete reporting of clinical information of the participants (32.31\%) were the most frequent reasons for assigning an unclear or high risk of bias. Appendix 1 shows a summary of the risk of bias assessment.

Patient Characteristics. The majority of the included patients were treated in European or North-American countries (94.6\%). The mean distribution of sex was $68.8 \%$ female ( $S D$ $=18.1 \%)$ The average mean age of the cohorts was 44.39 years $(S D=4.47)$. The median pain duration prior to treatment, reported in 40 of the included cohorts, was 76.8 months (range $=16-217.2$ ). Generally, patients were referred to the programme by their primary care physician or medical specialist. Table 1 provides a summary of the patient characteristics. The multimedia appendix includes a more extensive and searchable table. 
Table 1. Patient Characteristics.

\begin{tabular}{|c|c|c|c|c|c|c|c|c|}
\hline $\begin{array}{l}\text { Author (year) } \\
\text { cohort }\end{array}$ & $\mathbf{N}$ & $\begin{array}{c}\text { Dropout } \\
\text { (\%) }\end{array}$ & $\begin{array}{l}\text { Lost to } \\
\text { follow- } \\
\text { up (\%) }\end{array}$ & Nationality & $\begin{array}{c}\% \\
\text { Females }\end{array}$ & $\begin{array}{c}\text { Mean } \\
\text { age } \\
(S D)\end{array}$ & Patient group & $\begin{array}{c}\text { Mean pain } \\
\text { duration } \\
\text { (months) }\end{array}$ \\
\hline $\begin{array}{l}\text { Abbasi } \\
\text { (2012): a }\end{array}$ & 36 & 10 & 0 & Iran & 88 & $45(10)$ & low back pain & 107.5 \\
\hline $\begin{array}{l}\text { Abbasi } \\
(2012): \text { b }\end{array}$ & 36 & 0 & 16.67 & Iran & 88 & $45(10)$ & low back pain & 107.5 \\
\hline $\begin{array}{l}\text { Beaudreuil } \\
\text { (2010) }\end{array}$ & 39 & 0 & 0 & France & 28 & $\begin{array}{l}42.9 \\
(8.2)\end{array}$ & low back pain & 28.6 \\
\hline $\begin{array}{l}\text { Bendix } \\
\text { (1998)a }\end{array}$ & 238 & 14.55 & 6.38 & Denmark & 67.4 & 42 (NA) & $\begin{array}{c}\text { chronic low back } \\
\text { pain }\end{array}$ & NA \\
\hline $\begin{array}{l}\text { Bendix } \\
\text { (1998): b }\end{array}$ & 238 & 10.87 & 9.76 & Denmark & 70.27 & 41 (NA) & $\begin{array}{c}\text { chronic low back } \\
\text { pain }\end{array}$ & NA \\
\hline $\begin{array}{l}\text { Bendix } \\
(2000)\end{array}$ & 138 & 13.56 & 5.88 & Denmark & 66.1 & $\begin{array}{l}42.1 \\
(\mathrm{NA})\end{array}$ & $\begin{array}{c}\text { chronic low back } \\
\text { pain }\end{array}$ & NA \\
\hline $\begin{array}{l}\text { Bergström } \\
(2001)\end{array}$ & 156 & NA & NA & Sweden & 48 & $\begin{array}{l}42.5 \\
(9.5)\end{array}$ & $\begin{array}{c}\text { neck pain or } \\
\text { back pain }\end{array}$ & 38 \\
\hline $\begin{array}{l}\text { Bergström } \\
(2014)\end{array}$ & 43 & 9.3 & 12.82 & Sweden & 80 & 41 (NA) & $\begin{array}{l}\text { disabling chronic } \\
\text { pain }\end{array}$ & NA \\
\hline $\begin{array}{l}\text { Bileviciute- } \\
\text { Ljungar } \\
\text { (2014): a }\end{array}$ & 88 & NA & NA & Sweden & 84 & $\begin{array}{l}43.2 \\
(2.1)\end{array}$ & $\begin{array}{c}\text { persistent } \\
\text { musculoskeletal } \\
\text { pain }\end{array}$ & NA \\
\hline $\begin{array}{l}\text { Bileviciute- } \\
\text { Ljungar } \\
\text { (2014): b }\end{array}$ & 88 & NA & NA & Sweden & 89.47 & $\begin{array}{l}39.5 \\
(1.9)\end{array}$ & $\begin{array}{c}\text { persistent } \\
\text { musculoskeletal } \\
\text { pain }\end{array}$ & NA \\
\hline $\begin{array}{l}\text { Borys } \\
(2015)\end{array}$ & 155 & 0 & 59.35 & Germany & 55.6 & $\begin{array}{c}58.3 \\
(10.4)\end{array}$ & $\begin{array}{c}\text { chronic low back } \\
\text { pain }\end{array}$ & 217.2 \\
\hline $\begin{array}{l}\text { Brendbekken } \\
\text { (2016) }\end{array}$ & 284 & 2.84 & 37.96 & Norway & 53.9 & $\begin{array}{l}41.3 \\
(\mathrm{NA})\end{array}$ & $\begin{array}{l}\text { musculoskeletal } \\
\text { pain }\end{array}$ & NA \\
\hline $\begin{array}{l}\text { Cardosa } \\
(2012)\end{array}$ & 102 & 0 & 0 & Malaysia & 64.3 & $\begin{array}{l}42.87 \\
(9.87)\end{array}$ & chronic pain & 66.81 \\
\hline $\begin{array}{l}\text { De Rooij } \\
(2014)\end{array}$ & 138 & 9.77 & 5 & Netherlands & 95 & $\begin{array}{l}45.04 \\
(10.3)\end{array}$ & $\begin{array}{c}\text { chronic } \\
\text { widespread pain }\end{array}$ & 84 \\
\hline $\begin{array}{l}\text { Dysvik } \\
(2013)\end{array}$ & 117 & 11.11 & 8.65 & Norway & 79 & $\begin{array}{c}45 \\
(11.25)\end{array}$ & $\begin{array}{l}\text { chronic non- } \\
\text { malignant pain }\end{array}$ & 89.9 \\
\hline $\begin{array}{l}\text { Frost } \\
(2000)\end{array}$ & 129 & 1.63 & 19.83 & $\begin{array}{l}\text { United } \\
\text { Kingdom }\end{array}$ & 45 & $43(9)$ & constant pain & NA \\
\hline $\begin{array}{l}\text { Gantschnig } \\
(2017)\end{array}$ & 30 & 13.33 & -3.85 & Swiss & 43 & $\begin{array}{c}44.83 \\
(12.57)\end{array}$ & $\begin{array}{l}\text { ICD-10 diagnosis } \\
\text { of chronic } \\
\text { musculoskeletal } \\
\text { pain }\end{array}$ & NA \\
\hline
\end{tabular}


Table 1. Continued.

\begin{tabular}{|c|c|c|c|c|c|c|c|c|}
\hline $\begin{array}{l}\text { Author (year) } \\
\text { cohort }\end{array}$ & $\mathrm{N}$ & $\begin{array}{l}\text { Dropout } \\
\text { (\%) }\end{array}$ & $\begin{array}{l}\text { Lost to } \\
\text { follow- } \\
\text { up (\%) }\end{array}$ & Nationality & $\begin{array}{c}\% \\
\text { Females }\end{array}$ & $\begin{array}{l}\text { Mean } \\
\text { age } \\
(S D)\end{array}$ & Patient group & $\begin{array}{c}\text { Mean pain } \\
\text { duration } \\
\text { (months) }\end{array}$ \\
\hline $\begin{array}{l}\text { Gerdle } \\
(2016)\end{array}$ & 464 & NA & NA & Sweden & 81.6 & $\begin{array}{c}38.1 \\
(10.1)\end{array}$ & chronic pain & 83.8 \\
\hline $\begin{array}{l}\text { Grahn } \\
(2000)\end{array}$ & 236 & NA & NA & Sweden & 82 & $\begin{array}{l}44.3 \\
(9.1)\end{array}$ & $\begin{array}{c}\text { prolonged } \\
\text { musculoskeletal } \\
\text { disease as main } \\
\text { diagnosis }\end{array}$ & 68.4 \\
\hline $\begin{array}{l}\text { Gustafsson } \\
\text { (2002) }\end{array}$ & 23 & 4.35 & 22.73 & Sweden & 100 & $\begin{array}{c}43.8 \\
(10.7)\end{array}$ & $\begin{array}{l}\text { fibromyalgia } \\
\text { or widespread } \\
\text { chronic pain }\end{array}$ & 158.4 \\
\hline $\begin{array}{l}\text { Hafenbrack } \\
\text { (2013): a }\end{array}$ & 681 & 2.86 & 55.51 & Germany & 49.2 & $\begin{array}{l}45.81 \\
(9.22)\end{array}$ & back pain & NA \\
\hline $\begin{array}{l}\text { Hafenbrack } \\
\text { (2013): b }\end{array}$ & 681 & 3.18 & 55.26 & Germany & 55.3 & $\begin{array}{l}44.95 \\
(9.84)\end{array}$ & back Pain & NA \\
\hline $\begin{array}{l}\text { Haiduk } \\
(2017)\end{array}$ & 115 & NA & NA & Switzerland & 83.1 & $\begin{array}{c}40.3 \\
(12.3)\end{array}$ & $\begin{array}{l}\text { chronic neck } \\
\text { pain }\end{array}$ & 17.2 \\
\hline $\begin{array}{l}\text { Hållstam } \\
\text { (2016) }\end{array}$ & 42 & NA & NA & Sweden & 90.5 & $\begin{array}{c}43.6 \\
(15.7)\end{array}$ & $\begin{array}{l}\text { complex pain } \\
\text { problems }\end{array}$ & NA \\
\hline $\begin{array}{l}\text { Hazard } \\
(1989)\end{array}$ & 64 & 1.69 & 31.03 & USA & 36 & $37(4.3)$ & low back pain & NA \\
\hline $\begin{array}{l}\text { Hildebrandt } \\
\text { (1996) }\end{array}$ & 98 & 1.1 & 6.67 & Germany & 48.9 & $\begin{array}{l}41.7 \\
(8.7)\end{array}$ & $\begin{array}{c}\text { lumbal and } \\
\text { unspecific back } \\
\text { pain }\end{array}$ & NA \\
\hline $\begin{array}{l}\text { Huffman } \\
\text { (2019) }\end{array}$ & 2089 & 19.53 & 62.17 & USA & 65.3 & $\begin{array}{c}46.6 \\
(13.6)\end{array}$ & $\begin{array}{l}\text { chronic non } \\
\text { cancer pain }\end{array}$ & NA \\
\hline $\begin{array}{l}\text { Ibrahim } \\
\text { (2019) }\end{array}$ & 201 & 20.4 & 33.12 & Switzerland & 40.8 & $\begin{array}{c}39.98 \\
(10.06)\end{array}$ & non-specific LBP & NA \\
\hline $\begin{array}{l}\text { Jensen } \\
\text { (1997): a }\end{array}$ & 63 & 6.67 & 10.71 & Sweden & 100 & $43(9)$ & $\begin{array}{l}\text { nonspecific } \\
\text { spinal pain }\end{array}$ & 51 \\
\hline $\begin{array}{l}\text { Jensen } \\
\text { (1997): b }\end{array}$ & 63 & 6.06 & 6.45 & Sweden & 100 & $45(8)$ & $\begin{array}{l}\text { nonspecific } \\
\text { spinal pain }\end{array}$ & 44 \\
\hline $\begin{array}{l}\text { Kääpä } \\
(2006)\end{array}$ & 64 & 7.81 & 16.95 & Finland & 100 & $46(7.9)$ & low back pain & 16 \\
\hline $\begin{array}{l}\text { Koopman } \\
(2004)\end{array}$ & 68 & 5.88 & 20.31 & Netherlands & 47.1 & $\begin{array}{c}41.3 \\
(8.91)\end{array}$ & low back pain & 72 \\
\hline $\begin{array}{l}\text { Lemstra } \\
(2005)\end{array}$ & 43 & 16.28 & 2.78 & Canada & 86 & $\begin{array}{c}49.7 \\
(9.57)\end{array}$ & $\begin{array}{c}\text { chronic } \\
\text { widespread pain }\end{array}$ & 121.7 \\
\hline $\begin{array}{l}\text { Letzel } \\
(2019)\end{array}$ & 113 & 28.32 & 20.99 & Germany & 67.9 & $\begin{array}{c}59.7 \\
(10.7)\end{array}$ & $\begin{array}{c}\text { chronic neck } \\
\text { pain }\end{array}$ & NA \\
\hline
\end{tabular}


Table 1. Continued.

\begin{tabular}{|c|c|c|c|c|c|c|c|c|}
\hline $\begin{array}{l}\text { Author (year) } \\
\text { cohort }\end{array}$ & $\mathbf{N}$ & $\begin{array}{c}\text { Dropout } \\
\text { (\%) }\end{array}$ & $\begin{array}{l}\text { Lost to } \\
\text { follow- } \\
\text { up (\%) }\end{array}$ & Nationality & $\begin{array}{c}\% \\
\text { Females }\end{array}$ & $\begin{array}{l}\text { Mean } \\
\text { age } \\
(S D)\end{array}$ & Patient group & $\begin{array}{c}\text { Mean pain } \\
\text { duration } \\
\text { (months) }\end{array}$ \\
\hline $\begin{array}{l}\text { Mangels } \\
\text { (2009): a }\end{array}$ & 363 & 0 & 6.19 & Germany & 78.8 & 49.5 (9) & $\begin{array}{c}\text { musculoskeletal } \\
\text { pain }\end{array}$ & NA \\
\hline $\begin{array}{l}\text { Mangels } \\
\text { (2009): b }\end{array}$ & 363 & 0 & 6.72 & Germany & 75.6 & $\begin{array}{c}48.3 \\
(15.8)\end{array}$ & $\begin{array}{c}\text { musculoskeletal } \\
\text { pain }\end{array}$ & NA \\
\hline $\begin{array}{l}\text { Martín } \\
(2012)\end{array}$ & 180 & 10 & 13.89 & Spain & 93.46 & $\begin{array}{l}49.07 \\
(8.92)\end{array}$ & fibromyalgia & 174.22 \\
\hline $\begin{array}{l}\text { McAllister } \\
(2005)\end{array}$ & 276 & 20.65 & 54.79 & United States & 66.3 & $\begin{array}{l}44.7 \\
(9.7)\end{array}$ & $\begin{array}{l}\text { chronic non- } \\
\text { malignant pain }\end{array}$ & NA \\
\hline $\begin{array}{l}\text { Meng } \\
(2011)\end{array}$ & 382 & 11.17 & 16 & Germarny & 65.2 & $\begin{array}{l}50.2 \\
(7.6)\end{array}$ & $\begin{array}{c}\text { chronic low back } \\
\text { pain }\end{array}$ & NA \\
\hline $\begin{array}{l}\text { Merrick } \\
(2009)\end{array}$ & 255 & 1.79 & -1.21 & Sweden & 79 & 39 (NA) & $\begin{array}{c}\text { disabling chronic } \\
\text { pain }\end{array}$ & NA \\
\hline $\begin{array}{l}\text { Merrick } \\
(2012)\end{array}$ & 296 & NA & NA & Sweden & 76 & $\begin{array}{l}39.2 \\
(9.7)\end{array}$ & chronic pain & 62.4 \\
\hline $\begin{array}{l}\text { Monticone } \\
(2013)\end{array}$ & 90 & 0 & 0 & Italy & 60 & $\begin{array}{l}48.96 \\
(7.97)\end{array}$ & $\begin{array}{c}\text { chronic non- } \\
\text { specific low back } \\
\text { pain }\end{array}$ & 22.15 \\
\hline $\begin{array}{l}\text { Monticone } \\
(2016)\end{array}$ & 150 & 1.33 & 12.16 & Italy & 62.67 & $\begin{array}{c}53.2 \\
(11.1)\end{array}$ & $\begin{array}{c}\text { non-specific low } \\
\text { back pain }\end{array}$ & 21.7 \\
\hline $\begin{array}{l}\text { Nagel } \\
(2009)\end{array}$ & 351 & 38.96 & 0 & Germany & 59.3 & $\begin{array}{l}44.7 \\
\text { (NA) }\end{array}$ & $\begin{array}{c}\text { chronic } \\
\text { nonspecific back } \\
\text { pain }\end{array}$ & 140.4 \\
\hline $\begin{array}{l}\text { Nicholas } \\
\text { (2020): a }\end{array}$ & 140 & 10.61 & 28.81 & Australia & 51 & $\begin{array}{c}42.05 \\
(12.33)\end{array}$ & chronic pain & 67.16 \\
\hline $\begin{array}{l}\text { Nicholas } \\
(2020): \text { b }\end{array}$ & 140 & 8.11 & 41.18 & Australia & 55 & $\begin{array}{c}43.22 \\
(11.08)\end{array}$ & chronic pain & 77.71 \\
\hline $\begin{array}{l}\text { Olason } \\
(2004)\end{array}$ & 158 & 24.05 & NA & Iceland & 70.9 & $\begin{array}{l}39.5 \\
\text { (NA) }\end{array}$ & chronic pain & NA \\
\hline $\begin{array}{l}\text { Oslund } \\
\text { (2009) }\end{array}$ & 108 & 0 & 42.5 & USA & 70.4 & $\begin{array}{c}55 \\
(11.47)\end{array}$ & chronic pain & 110.44 \\
\hline $\begin{array}{l}\text { Persson } \\
\text { (2012) }\end{array}$ & 813 & 13.53 & 27.6 & Sweden & 79 & 40 (9.6) & $\begin{array}{c}\text { musculoskeletal } \\
\text { pain }\end{array}$ & 49.2 \\
\hline $\begin{array}{l}\text { Pietilä- } \\
\text { Holmner } \\
(2020)\end{array}$ & 467 & NA & NA & Sweden & 85.5 & $\begin{array}{c}43.6 \\
(10.8)\end{array}$ & $\begin{array}{c}\text { musculoskeletal } \\
\text { chronic pain }\end{array}$ & NA \\
\hline $\begin{array}{l}\text { Reck } \\
(2017)\end{array}$ & 71 & NA & NA & Switzerland & 54 & $\begin{array}{c}43.59 \\
(11.84)\end{array}$ & $\begin{array}{c}\text { chronic non- } \\
\text { specific low back } \\
\text { pain }\end{array}$ & NA \\
\hline
\end{tabular}


Table 1. Continued.

\begin{tabular}{|c|c|c|c|c|c|c|c|c|}
\hline $\begin{array}{l}\text { Author (year) } \\
\text { cohort }\end{array}$ & $\mathrm{N}$ & $\begin{array}{c}\text { Dropout } \\
\text { (\%) }\end{array}$ & $\begin{array}{l}\text { Lost to } \\
\text { follow- } \\
\text { up (\%) }\end{array}$ & Nationality & $\begin{array}{c}\% \\
\text { Females }\end{array}$ & $\begin{array}{l}\text { Mean } \\
\text { age } \\
(S D)\end{array}$ & Patient group & $\begin{array}{c}\text { Mean pain } \\
\text { duration } \\
\text { (months) }\end{array}$ \\
\hline $\begin{array}{l}\text { Richardson } \\
\text { (1994) }\end{array}$ & 109 & 9.17 & -10.1 & $\begin{array}{l}\text { United } \\
\text { Kingdom }\end{array}$ & 68 & $45(10)$ & chronic pain & 128 \\
\hline $\begin{array}{l}\text { Roche- } \\
\text { Leboucher } \\
(2011)\end{array}$ & 132 & NA & NA & France & 32.4 & $\begin{array}{l}40.8 \\
(7.4)\end{array}$ & $\begin{array}{c}\text { non-specific low } \\
\text { back pain }\end{array}$ & NA \\
\hline $\begin{array}{l}\text { Semrau } \\
(2015)\end{array}$ & 554 & 5 & 27.44 & Germany & 54.1 & $48.9(8)$ & low back pain & NA \\
\hline $\begin{array}{l}\text { Silvemark } \\
(2014)\end{array}$ & 164 & 13.64 & 48.87 & Sweden & 69.5 & $\begin{array}{c}37.4 \\
(9.07)\end{array}$ & $\begin{array}{l}\text { long-term non- } \\
\text { malignant pain }\end{array}$ & 68.4 \\
\hline $\begin{array}{l}\text { Smeets } \\
\text { (2008): a }\end{array}$ & 223 & 5.17 & 5.45 & Netherlands & 58.6 & $\begin{array}{l}42.52 \\
(9.67)\end{array}$ & $\begin{array}{c}\text { non-specific } \\
\text { low-back pain }\end{array}$ & 68.33 \\
\hline $\begin{array}{l}\text { Smeets } \\
\text { (2008): b }\end{array}$ & 223 & 9.84 & 3.64 & Netherlands & 37.7 & $\begin{array}{c}40.67 \\
(10.14)\end{array}$ & $\begin{array}{c}\text { non-specific } \\
\text { low-back pain }\end{array}$ & 56.13 \\
\hline $\begin{array}{l}\text { Spinhoven } \\
(2004)\end{array}$ & 148 & 11.97 & 12.62 & Netherlands & 63.5 & $\begin{array}{l}39.8 \\
(9.1)\end{array}$ & low back pain & 117.6 \\
\hline $\begin{array}{l}\text { Stein } \\
(2013)\end{array}$ & 59 & 0 & 13.56 & Sweden & 86 & $48(7.8)$ & $\begin{array}{l}\text { chronic non- } \\
\text { cancer pain }\end{array}$ & NA \\
\hline $\begin{array}{l}\text { Steinmetz } \\
\text { (2019) }\end{array}$ & 276 & 9.78 & 56.22 & Germany & 57.4 & $\begin{array}{c}53.4 \\
(10.6)\end{array}$ & $\begin{array}{l}\text { chronic spinal } \\
\text { pain }\end{array}$ & \\
\hline $\begin{array}{l}\text { Strobel } \\
(1998)\end{array}$ & 32 & NA & NA & Germany & 87.5 & $49(7.5)$ & $\begin{array}{l}\text { fibromyalgia } \\
\text { (ACR criteria) }\end{array}$ & 92.4 \\
\hline $\begin{array}{l}\text { Tavafian } \\
\text { (2011) }\end{array}$ & 197 & 4.12 & 25.81 & Iran & 73.2 & $\begin{array}{c}44.6 \\
(10.2)\end{array}$ & $\begin{array}{c}\text { chronic low back } \\
\text { pain }\end{array}$ & 75.9 \\
\hline $\begin{array}{l}\text { Thieme } \\
\text { (2003) }\end{array}$ & 61 & 4.76 & 0 & Germany & 100 & $\begin{array}{l}46.61 \\
(8.67)\end{array}$ & fibromyalgia & 204.6 \\
\hline $\begin{array}{l}\text { Van der Maas } \\
(2015): a\end{array}$ & 114 & 42.11 & 21.21 & Netherlands & 71.1 & $\begin{array}{c}45.4 \\
(11.1)\end{array}$ & $\begin{array}{c}\text { chronic } \\
\text { musculoskeletal } \\
\text { pain }\end{array}$ & NA \\
\hline $\begin{array}{l}\text { Van der Maas } \\
\text { (2015): b }\end{array}$ & 114 & 8.16 & 48.89 & Netherlands & 91.8 & $\begin{array}{c}38.6 \\
(11.1)\end{array}$ & $\begin{array}{c}\text { chronic } \\
\text { musculoskeletal } \\
\text { pain }\end{array}$ & NA \\
\hline $\begin{array}{l}\text { van Hooff } \\
(2010)\end{array}$ & 107 & 23.36 & 0 & Netherlands & 57 & $\begin{array}{l}44.1 \\
(8.4)\end{array}$ & low back pain & 147.6 \\
\hline $\begin{array}{l}\text { van Wilgen } \\
(2009)\end{array}$ & 32 & 18.75 & 0 & Netherlands & 73 & $42(11)$ & chronic pain & 96 \\
\hline $\begin{array}{l}\text { Vendrig } \\
(2000)\end{array}$ & 147 & NA & NA & Netherlands & 31 & $\begin{array}{l}41.6 \\
(8.5)\end{array}$ & $\begin{array}{c}\text { chronic low back } \\
\text { pain }\end{array}$ & 46.3 \\
\hline
\end{tabular}


Table 1. Continued.

\begin{tabular}{|c|c|c|c|c|c|c|c|c|}
\hline $\begin{array}{l}\text { Author (year) } \\
\text { cohort }\end{array}$ & $\mathbf{N}$ & $\begin{array}{c}\text { Dropout } \\
\text { (\%) }\end{array}$ & $\begin{array}{l}\text { Lost to } \\
\text { follow- } \\
\text { up (\%) }\end{array}$ & Nationality & $\begin{array}{c}\% \\
\text { Females }\end{array}$ & $\begin{array}{l}\text { Mean } \\
\text { age } \\
(S D)\end{array}$ & Patient group & $\begin{array}{c}\text { Mean pain } \\
\text { duration } \\
\text { (months) }\end{array}$ \\
\hline $\begin{array}{l}\text { Verkerk } \\
\text { (2011) }\end{array}$ & 1760 & 3.64 & 43.1 & Netherlands & 74.26 & $\begin{array}{c}40.1 \\
(10.6)\end{array}$ & $\begin{array}{l}\text { Chronic non- } \\
\text { specific low back } \\
\text { pain }\end{array}$ & 92.4 \\
\hline $\begin{array}{l}\text { Volker } \\
(2017)\end{array}$ & 165 & 6.67 & 22.08 & Netherlands & 87 & $\begin{array}{c}44.1 \\
(12.9)\end{array}$ & $\begin{array}{c}\text { chronic } \\
\text { musculoskeletal } \\
\text { pain }\end{array}$ & NA \\
\hline $\begin{array}{l}\text { Vowles } \\
\text { (2011) }\end{array}$ & 187 & 8.56 & 36.84 & $\begin{array}{l}\text { United } \\
\text { Kingdom }\end{array}$ & 64.2 & $\begin{array}{c}47.3 \\
(11.4)\end{array}$ & chronic pain & 96 \\
\hline $\begin{array}{l}\text { Wagner } \\
\text { (2011) }\end{array}$ & 142 & 0 & 0 & Canada & 63.64 & $\begin{array}{c}49.16 \\
(10.03)\end{array}$ & chronic pain & 32.99 \\
\hline $\begin{array}{l}\text { Westman } \\
\text { (2006) }\end{array}$ & 91 & NA & NA & Sweden & 70 & $\begin{array}{l}41.5 \\
\text { (NA) }\end{array}$ & $\begin{array}{c}\text { musculoskeletal } \\
\text { pain and } \\
\text { disability }\end{array}$ & NA \\
\hline $\begin{array}{l}\text { Zhuk } \\
(2018)\end{array}$ & 59 & NA & 85.68 & Germany & 62.7 & $\begin{array}{c}45.27 \\
(10.34)\end{array}$ & low back pain & NA \\
\hline
\end{tabular}


Intervention characteristics. The treatment aims of the included programmes often involved multiple objectives, such as increasing physical activity, return to work, or the acquisition of pain self-management skills. The median time span of the treatment duration was 5 weeks (range 1 - 15), with a mean intensity of 96.76 hours $(S D=52.83)$. Twentytwo cohorts (29.73\%) included, at least partly, inpatient treatment programmes and 2 cohorts (2.7\%) solely provided treatments to individuals, whereas the other cohorts at least partially provided treatment to groups. The majority of interdisciplinary treatment was provided in secondary or tertiary care settings, such as hospitals or rehabilitation centers, with a community center (1 cohort), hotel (1 cohort) and a primary care setting (3 cohorts) as exceptions. Exercise (93\%), education (89\%), relaxation (80\%), self-management skills (73\%), and (cognitive) behavioural treatment (69.\%) were included in the majority of the treatment programmes, whereas body awareness (26\%), graded activity (14\%), workplace advice (16\%), pharmacological treatment (15\%), and team meetings that included the patient (11\%) were less frequently reported. The median number of these specific treatment modalities was 5 (range $=2-8$ ) per cohort. Many cohorts also included modalities that were categorized as 'other', such as assertiveness training, spinal mobilizations, group discussions, and assistance with withdrawal from pain medication. The median number of involved healthcare professionals was 4 (range $=3-7$ ), with physical therapists $(97 \%)$, psychologists (93\%), physicians (85\%), occupational therapists (53\%), nurses (34\%) and social workers (32\%) as respective frequencies. Other involved healthcare providers (described in $57 \%$ of the cohorts) included nutritionists, massage therapists and Qigong instructors.

Follow-up treatment sessions were described in 22 (41\%) of the cohorts and mainly consisted of group refresher meetings or follow-up phone calls. Four cohorts included an extensive follow-up module where parts of the treatment programme were continued for a prolonged period (Monticone et al., 2016; Monticone et al., 2013; Tavafian et al., 2011; Westman et al., 2006). In total, $81 \%$ of the included studies provided low tailoring, $11 \%$ medium tailoring and $8 \%$ high tailoring. Table 2 depicts a general overview of the intervention characteristics, but the full table is displayed in the multimedia appendix. 
Table 2. Intervention characteristics.

\begin{tabular}{|c|c|c|c|c|c|c|c|c|c|c|c|c|}
\hline \multirow[b]{2}{*}{ Cohort } & \multirow[b]{2}{*}{$\begin{array}{l}\text { Treatment } \\
\text { modalities }\end{array}$} & \multicolumn{6}{|c|}{ Healthcare providers } & \multirow{2}{*}{$\begin{array}{c}\text { Type of } \\
\text { contact } \\
\text { (group size) }\end{array}$} & \multirow{2}{*}{$\begin{array}{l}\text { Time } \\
\text { span } \\
\text { (wks) }\end{array}$} & \multirow[b]{2}{*}{$\begin{array}{c}\text { Duration } \\
\text { (hours) }\end{array}$} & \multirow[b]{2}{*}{$\begin{array}{c}\text { Level of } \\
\text { tailoring }\end{array}$} & \multirow[b]{2}{*}{$\begin{array}{c}\text { follow } \\
\text { up? }\end{array}$} \\
\hline & & $\overrightarrow{\mathrm{E}}$ & 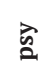 & 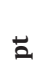 & $\ddot{0}$ & 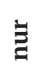 & 空 & & & & & \\
\hline $\begin{array}{l}\text { Abbasi } \\
(2012): a\end{array}$ & $\begin{array}{c}\text { ed, bt, re, sm, } \\
\text { oth }\end{array}$ & $\mathrm{X}$ & $\mathrm{X}$ & $\mathrm{X}$ & - & - & - & mixed (6) & 7 & 15 & low & no \\
\hline $\begin{array}{l}\text { Abbasi } \\
(2012): \text { b }\end{array}$ & $\begin{array}{c}\text { ed, bt, re, sm, } \\
\text { oth }\end{array}$ & $\mathrm{X}$ & $\mathrm{X}$ & $\mathrm{X}$ & - & - & - & mixed (6) & 7 & 16 & low & no \\
\hline $\begin{array}{l}\text { Beaudreuil } \\
\text { (2010) }\end{array}$ & ex, re, oth & $\mathrm{X}$ & - & $\mathrm{X}$ & $\mathrm{X}$ & - & $\mathrm{X}$ & $\begin{array}{l}\text { group } \\
(2-6)\end{array}$ & 5 & 138 & low & no \\
\hline $\begin{array}{l}\text { Bendix } \\
\text { (1998)a }\end{array}$ & $\begin{array}{c}\text { ed, ex, bt, re, } \\
\text { oth }\end{array}$ & $\mathrm{X}$ & $\mathrm{X}$ & $\mathrm{X}$ & $\mathrm{X}$ & - & $\mathrm{X}$ & $\begin{array}{c}\text { group } \\
(6-8)\end{array}$ & 3 & 135 & low & no \\
\hline $\begin{array}{l}\text { Bendix } \\
\text { (1998): b }\end{array}$ & $\begin{array}{c}\text { ed, ex, bt, re, } \\
\text { oth }\end{array}$ & $\mathrm{X}$ & $\mathrm{X}$ & $\mathrm{X}$ & $\mathrm{X}$ & - & $\mathrm{X}$ & $\begin{array}{c}\text { group } \\
(6-8)\end{array}$ & 3 & 135 & low & no \\
\hline $\begin{array}{l}\text { Bendix } \\
(2000)\end{array}$ & $\begin{array}{c}\text { ed, ex, re, sm, } \\
\text { oth }\end{array}$ & $\mathrm{X}$ & $\mathrm{X}$ & $\mathrm{X}$ & $\mathrm{X}$ & - & $\mathrm{X}$ & $\begin{array}{c}\text { group } \\
(6-8)\end{array}$ & 3 & 135 & low & no \\
\hline $\begin{array}{l}\text { Bergström } \\
(2001)\end{array}$ & $\begin{array}{c}\text { ed, ex, re, ba, } \\
\text { oth }\end{array}$ & - & - & $\mathrm{X}$ & - & - & - & $\begin{array}{l}\text { group } \\
(14)\end{array}$ & 4 & 160 & medium & no \\
\hline $\begin{array}{l}\text { Bergström } \\
(2014)\end{array}$ & $\begin{array}{c}\text { ed, ex, re, sm, } \\
\text { ba, te, oth }\end{array}$ & $\mathrm{X}$ & $\mathrm{X}$ & $\mathrm{X}$ & $\mathrm{X}$ & - & $\mathrm{X}$ & $\begin{array}{c}\text { group } \\
(10-12)\end{array}$ & 5 & NA & low & no \\
\hline $\begin{array}{l}\text { Bileviciute- } \\
\text { Ljungar } \\
\text { (2014): a }\end{array}$ & $\begin{array}{c}\text { ed, ex, sm, wo, } \\
\text { oth }\end{array}$ & $\mathrm{X}$ & $\mathrm{X}$ & $\mathrm{X}$ & $\mathrm{X}$ & $\mathrm{X}$ & $\mathrm{X}$ & $\begin{array}{l}\text { mixed } \\
(8)\end{array}$ & 8 & 143 & low & no \\
\hline $\begin{array}{l}\text { Bileviciute- } \\
\text { Ljungar } \\
\text { (2014): b }\end{array}$ & $\begin{array}{c}\text { ed, ex, sm, wo, } \\
\text { oth }\end{array}$ & $\mathrm{X}$ & $\mathrm{X}$ & $\mathrm{X}$ & $\mathrm{X}$ & $\mathrm{X}$ & $\mathrm{X}$ & $\begin{array}{l}\text { mixed } \\
(8)\end{array}$ & 8 & 143 & low & no \\
\hline $\begin{array}{l}\text { Borys } \\
(2015)\end{array}$ & $\begin{array}{c}\text { ed, ex, bt, ph, } \\
\text { ba, te }\end{array}$ & $\mathrm{X}$ & $\mathrm{X}$ & $\mathrm{X}$ & - & - & $\mathrm{X}$ & $\begin{array}{c}\text { mixed } \\
\text { (NA) }\end{array}$ & 3 & 168 & low & no \\
\hline $\begin{array}{l}\text { Brendbekken } \\
\text { (2016) }\end{array}$ & ex, sm, te & $\mathrm{X}$ & - & $\mathrm{X}$ & - & - & $\mathrm{X}$ & $\begin{array}{l}\text { individual } \\
\text { (NA) }\end{array}$ & NA & 8 & low & yes \\
\hline $\begin{array}{l}\text { Cardosa } \\
\text { (2012) }\end{array}$ & $\begin{array}{c}\text { ed, ex, bt, re, } \\
\text { sm, oth }\end{array}$ & $\mathrm{X}$ & $\mathrm{X}$ & $\mathrm{X}$ & - & $\mathrm{X}$ & - & $\begin{array}{l}\text { group } \\
\text { (NA) }\end{array}$ & 2 & 60 & low & no \\
\hline $\begin{array}{l}\text { De Rooij } \\
(2014)\end{array}$ & $\begin{array}{c}\text { ed, ex, bt, re, } \\
\text { sm, oth }\end{array}$ & $\mathrm{X}$ & $\mathrm{X}$ & $\mathrm{X}$ & $\mathrm{X}$ & - & $\mathrm{X}$ & $\begin{array}{l}\text { mixed } \\
(\mathrm{NA})\end{array}$ & 7 & 49 & high & yes \\
\hline $\begin{array}{l}\text { Dysvik } \\
(2013)\end{array}$ & $\begin{array}{c}\text { ed, ex, sm, ba, } \\
\text { oth }\end{array}$ & $\mathrm{X}$ & $\mathrm{X}$ & $\mathrm{X}$ & - & $\mathrm{X}$ & - & $\begin{array}{l}\text { group } \\
(8-12)\end{array}$ & 8 & 45 & low & yes \\
\hline $\begin{array}{l}\text { Frost } \\
(2000)\end{array}$ & ed, ex, re, oth & $\mathrm{X}$ & $\mathrm{X}$ & $\mathrm{X}$ & - & - & - & $\begin{array}{l}\text { group } \\
\text { (NA) }\end{array}$ & 3 & 98 & low & yes \\
\hline $\begin{array}{l}\text { Gantschnig } \\
\text { (2017) }\end{array}$ & $\begin{array}{c}\text { ed, ex, bt, re, } \\
\text { sm, ba, wo, } \\
\text { oth }\end{array}$ & $\mathrm{X}$ & $\mathrm{X}$ & $\mathrm{X}$ & $\mathrm{X}$ & $\mathrm{X}$ & $\mathrm{X}$ & $\begin{array}{l}\text { mixed } \\
\text { (NA) }\end{array}$ & 12 & 108 & medium & no \\
\hline
\end{tabular}


Table 2. Continued.

\begin{tabular}{|c|c|c|c|c|c|c|c|c|c|c|c|c|}
\hline \multirow[b]{2}{*}{ Cohort } & \multirow[b]{2}{*}{$\begin{array}{l}\text { Treatment } \\
\text { modalities }\end{array}$} & \multicolumn{6}{|c|}{ Healthcare providers } & \multirow{2}{*}{$\begin{array}{c}\text { Type of } \\
\text { contact } \\
\text { (group size) }\end{array}$} & \multirow{2}{*}{$\begin{array}{l}\text { Time } \\
\text { span } \\
\text { (wks) }\end{array}$} & \multirow[b]{2}{*}{$\begin{array}{c}\text { Duration } \\
\text { (hours) }\end{array}$} & \multirow[b]{2}{*}{$\begin{array}{l}\text { Level of } \\
\text { tailoring }\end{array}$} & \multirow[b]{2}{*}{$\begin{array}{c}\text { follow } \\
\text { up? }\end{array}$} \\
\hline & & $\frac{\vec{a}}{2}$ & 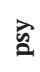 & $\overrightarrow{\mathrm{a}}$ & ¿ & $\stackrel{\Xi}{\Xi}$ & 总 & & & & & \\
\hline $\begin{array}{l}\text { Gerdle } \\
(2016)\end{array}$ & ed, bt, sm, oth & $\mathrm{X}$ & $\mathrm{X}$ & $\mathrm{X}$ & $\mathrm{X}$ & - & $\mathrm{X}$ & $\begin{array}{l}\text { group } \\
(6-9)\end{array}$ & 7 & 140 & medium & no \\
\hline $\begin{array}{l}\text { Grahn } \\
(2000)\end{array}$ & $\begin{array}{c}\text { ed, ex, bt, re, } \\
\text { sm, ba, wo, } \\
\text { oth }\end{array}$ & $\mathrm{X}$ & - & $\mathrm{X}$ & $\mathrm{X}$ & $\mathrm{X}$ & - & $\begin{array}{l}\text { mixed } \\
\text { (NA) }\end{array}$ & 4 & 120 & high & yes \\
\hline $\begin{array}{l}\text { Gustafsson } \\
(2002)\end{array}$ & $\begin{array}{c}\text { ed, ex, re, ba, } \\
\text { oth }\end{array}$ & $\mathrm{X}$ & $\mathrm{X}$ & - & - & $\mathrm{X}$ & $\mathrm{X}$ & $\begin{array}{l}\text { mixed } \\
(7-8)\end{array}$ & 12 & 96 & low & yes \\
\hline $\begin{array}{l}\text { Hafenbrack } \\
\text { (2013): a }\end{array}$ & $\begin{array}{c}\text { ed, ex, re, sm, } \\
\text { ph, wo }\end{array}$ & $\mathrm{X}$ & $\mathrm{X}$ & $\mathrm{X}$ & - & - & - & $\begin{array}{l}\text { group } \\
\text { (NA) }\end{array}$ & 4 & 120 & low & no \\
\hline $\begin{array}{l}\text { Hafenbrack } \\
\text { (2013): b }\end{array}$ & $\begin{array}{c}\text { ed, ex, re, sm, } \\
\text { ph, wo }\end{array}$ & $\mathrm{X}$ & $\mathrm{X}$ & $\mathrm{X}$ & - & - & - & $\begin{array}{l}\text { group } \\
\text { (NA) }\end{array}$ & 4 & 120 & low & no \\
\hline $\begin{array}{l}\text { Haiduk } \\
\text { (2017) }\end{array}$ & ex, bt, re, sm & $\mathrm{X}$ & $\mathrm{X}$ & $\mathrm{X}$ & $\mathrm{X}$ & - & - & $\begin{array}{l}\text { mixed } \\
\text { (NA) }\end{array}$ & 4 & 108 & low & no \\
\hline $\begin{array}{l}\text { Hållstam } \\
\text { (2016) }\end{array}$ & $\begin{array}{c}\text { ex, bt, re, sm, } \\
\text { oth }\end{array}$ & $\mathrm{X}$ & $\mathrm{X}$ & $\mathrm{X}$ & - & $\mathrm{X}$ & - & $\begin{array}{l}\text { mixed } \\
\text { (NA) }\end{array}$ & 13 & 65 & low & no \\
\hline $\begin{array}{l}\text { Hazard } \\
(1989)\end{array}$ & $\begin{array}{l}\text { ed, ex, bt, re, } \\
\text { sm, oth }\end{array}$ & - & $\mathrm{X}$ & $\mathrm{X}$ & $\mathrm{X}$ & - & - & $\begin{array}{l}\text { mixed } \\
(\mathrm{NA})\end{array}$ & 3 & 53 & low & no \\
\hline $\begin{array}{l}\text { Hildebrandt } \\
\text { (1996) }\end{array}$ & $\begin{array}{c}\text { ed, ex, bt, re, } \\
\text { sm, wo }\end{array}$ & $\mathrm{X}$ & $\mathrm{X}$ & $\mathrm{X}$ & - & $\mathrm{X}$ & $\mathrm{X}$ & $\begin{array}{l}\text { group } \\
(8-10)\end{array}$ & 11 & 207 & medium & no \\
\hline $\begin{array}{l}\text { Huffman } \\
\text { (2019) }\end{array}$ & ed, ex, bt, oth & - & $\mathrm{X}$ & $\mathrm{X}$ & $\mathrm{X}$ & $\mathrm{X}$ & - & $\begin{array}{l}\text { mixed } \\
\text { (NA) }\end{array}$ & 3.5 & 166 & low & yes \\
\hline $\begin{array}{l}\text { Ibrahim } \\
\text { (2019) }\end{array}$ & $\begin{array}{c}\text { ed, ex, bt, re, } \\
\text { sm, oth }\end{array}$ & $\mathrm{X}$ & $\mathrm{X}$ & $\mathrm{X}$ & $\mathrm{X}$ & - & - & $\begin{array}{l}\text { group } \\
(4-6)\end{array}$ & 4 & 100 & low & yes \\
\hline $\begin{array}{l}\text { Jensen } \\
\text { (1997): a }\end{array}$ & $\begin{array}{l}\text { ed, ex, bt, re, } \\
\text { sm, oth }\end{array}$ & $\mathrm{X}$ & $\mathrm{X}$ & $\mathrm{X}$ & - & $\mathrm{X}$ & - & $\begin{array}{l}\text { group } \\
\text { (NA) }\end{array}$ & 5 & NA & low & yes \\
\hline $\begin{array}{l}\text { Jensen } \\
\text { (1997): b }\end{array}$ & $\begin{array}{c}\text { ed, ex, bt, re, } \\
\text { sm, oth }\end{array}$ & $\mathrm{X}$ & $\mathrm{X}$ & $\mathrm{X}$ & - & $\mathrm{X}$ & - & $\begin{array}{l}\text { group } \\
\text { (NA) }\end{array}$ & 5 & NA & low & yes \\
\hline $\begin{array}{l}\text { Kääpä } \\
\text { (2006) }\end{array}$ & $\begin{array}{c}\text { ed, ex, bt, re, } \\
\text { ph, wo }\end{array}$ & $\mathrm{X}$ & $\mathrm{X}$ & $\mathrm{X}$ & $\mathrm{X}$ & - & - & $\begin{array}{c}\text { mixed } \\
(6-8)\end{array}$ & 8 & 70 & low & no \\
\hline $\begin{array}{l}\text { Koopman } \\
\text { (2004) }\end{array}$ & $\begin{array}{c}\text { ex, ga, bt, re, } \\
\text { oth }\end{array}$ & $\mathrm{X}$ & $\mathrm{X}$ & $\mathrm{X}$ & - & - & - & $\begin{array}{l}\text { mixed } \\
(6-10)\end{array}$ & 12 & 216 & low & no \\
\hline $\begin{array}{l}\text { Lemstra } \\
(2005)\end{array}$ & $\begin{array}{c}\text { ed, ex, re, sm, } \\
\text { oth }\end{array}$ & $\mathrm{X}$ & $\mathrm{X}$ & $\mathrm{X}$ & - & - & - & $\begin{array}{l}\text { mixed } \\
\text { (NA) }\end{array}$ & 6 & 33 & low & no \\
\hline $\begin{array}{l}\text { Letzel } \\
(2019)\end{array}$ & $\begin{array}{c}\text { ed, ex, bt, re, } \\
\text { sm, oth }\end{array}$ & $\mathrm{X}$ & $\mathrm{X}$ & $\mathrm{X}$ & $\mathrm{X}$ & - & - & $\begin{array}{l}\text { group } \\
(5-10)\end{array}$ & 3 & 44 & low & no \\
\hline $\begin{array}{l}\text { Mangels } \\
\text { (2009): a }\end{array}$ & $\begin{array}{l}\text { ed, ex, re, } \\
\text { sm, ph }\end{array}$ & - & $\mathrm{X}$ & $\mathrm{X}$ & $\mathrm{X}$ & - & - & $\begin{array}{l}\text { mixed } \\
(10-12)\end{array}$ & NA & 167 & low & no \\
\hline
\end{tabular}


Table 2. Continued.

\begin{tabular}{|c|c|c|c|c|c|c|c|c|c|c|c|c|}
\hline \multirow[b]{2}{*}{ Cohort } & \multirow[b]{2}{*}{$\begin{array}{l}\text { Treatment } \\
\text { modalities }\end{array}$} & \multicolumn{6}{|c|}{ Healthcare providers } & \multirow{2}{*}{$\begin{array}{c}\text { Type of } \\
\text { contact } \\
\text { (group size) }\end{array}$} & \multirow{2}{*}{$\begin{array}{l}\text { Time } \\
\text { span } \\
\text { (wks) }\end{array}$} & \multirow[b]{2}{*}{$\begin{array}{c}\text { Duration } \\
\text { (hours) }\end{array}$} & \multirow[b]{2}{*}{$\begin{array}{l}\text { Level of } \\
\text { tailoring }\end{array}$} & \multirow[b]{2}{*}{$\begin{array}{c}\text { follow } \\
\text { up? }\end{array}$} \\
\hline & & $\vec{a}$ & 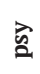 & $\ddot{z}$ & $\ddot{0}$ & $\stackrel{\Xi}{\Xi}$ & 空 & & & & & \\
\hline $\begin{array}{l}\text { Mangels } \\
\text { (2009): b }\end{array}$ & $\begin{array}{l}\text { ed, ex, re, } \\
\text { sm, ph }\end{array}$ & - & $\mathrm{X}$ & $\mathrm{X}$ & $\mathrm{X}$ & - & - & $\begin{array}{c}\text { mixed } \\
(10-12)\end{array}$ & NA & 167 & low & yes \\
\hline $\begin{array}{l}\text { Martín } \\
(2012)\end{array}$ & $\begin{array}{c}\text { ed, ex, bt, re, } \\
\text { sm, ph }\end{array}$ & $\mathrm{X}$ & $\mathrm{X}$ & $\mathrm{X}$ & - & - & - & $\begin{array}{l}\text { group } \\
(12)\end{array}$ & 6 & 21 & low & no \\
\hline $\begin{array}{l}\text { McAllister } \\
(2005)\end{array}$ & ex, re, sm, oth & $\mathrm{X}$ & - & $\mathrm{X}$ & - & $\mathrm{X}$ & - & $\begin{array}{l}\text { group } \\
\text { (NA) }\end{array}$ & 4 & 80 & low & yes \\
\hline $\begin{array}{l}\text { Meng } \\
(2011)\end{array}$ & ed, ex, sm & - & $\mathrm{X}$ & $\mathrm{X}$ & - & - & - & $\begin{array}{l}\text { group } \\
(7-15)\end{array}$ & 3 & 163 & low & no \\
\hline $\begin{array}{l}\text { Merrick } \\
(2009)\end{array}$ & $\begin{array}{c}\text { ed, ex, bt, re, } \\
\text { sm, ba, oth }\end{array}$ & $\mathrm{X}$ & $\mathrm{X}$ & $\mathrm{X}$ & $\mathrm{X}$ & - & $\mathrm{X}$ & $\begin{array}{l}\text { mixed } \\
\text { (NA) }\end{array}$ & 5 & 67 & low & no \\
\hline $\begin{array}{l}\text { Merrick } \\
(2012)\end{array}$ & $\begin{array}{c}\text { ed, ex, bt, re, } \\
\text { sm, ba, te, oth }\end{array}$ & $\mathrm{X}$ & $\mathrm{X}$ & $\mathrm{X}$ & $\mathrm{X}$ & - & $\mathrm{X}$ & $\begin{array}{l}\text { group } \\
(6-8)\end{array}$ & 4 & 65 & medium & no \\
\hline $\begin{array}{l}\text { Monticone } \\
(2013)\end{array}$ & $\begin{array}{c}\text { ed, ex, bt, sm, } \\
\text { oth }\end{array}$ & $\mathrm{X}$ & $\mathrm{X}$ & $\mathrm{X}$ & - & - & - & $\begin{array}{l}\text { individual } \\
\text { (NA) }\end{array}$ & 5 & 15 & low & yes \\
\hline $\begin{array}{l}\text { Monticone } \\
(2016)\end{array}$ & $\begin{array}{c}\text { ed, ex, bt, re, } \\
\text { sm, oth }\end{array}$ & $\mathrm{X}$ & $\mathrm{X}$ & $\mathrm{X}$ & - & - & - & $\begin{array}{l}\text { group } \\
(5)\end{array}$ & 5 & 15 & low & yes \\
\hline $\begin{array}{l}\text { Nagel } \\
(2009)\end{array}$ & ed, ex, bt, re & $\mathrm{X}$ & $\mathrm{X}$ & $\mathrm{X}$ & - & $\mathrm{X}$ & - & $\begin{array}{l}\text { group } \\
(8-9)\end{array}$ & 3.5 & 89 & low & no \\
\hline $\begin{array}{l}\text { Nicholas } \\
\text { (2020): a }\end{array}$ & $\begin{array}{c}\text { ed, ex, bt, sm, } \\
\text { oth }\end{array}$ & $\mathrm{X}$ & $\mathrm{X}$ & $\mathrm{X}$ & - & $\mathrm{X}$ & - & $\begin{array}{l}\text { group } \\
(8-10)\end{array}$ & 3 & 115 & low & no \\
\hline $\begin{array}{l}\text { Nicholas } \\
\text { (2020): b }\end{array}$ & $\begin{array}{c}\text { ed, ex, bt, re, } \\
\text { sm }\end{array}$ & $\mathrm{X}$ & $\mathrm{X}$ & $\mathrm{X}$ & - & $\mathrm{X}$ & - & $\begin{array}{l}\text { group } \\
(8-10)\end{array}$ & 3 & 115 & low & no \\
\hline $\begin{array}{l}\text { Olason } \\
(2004)\end{array}$ & $\begin{array}{l}\text { ed, ex, bt, re, } \\
\text { sm, ph, ba, te, } \\
\text { oth }\end{array}$ & $\mathrm{X}$ & $\mathrm{X}$ & $\mathrm{X}$ & $\mathrm{X}$ & $\mathrm{X}$ & $\mathrm{X}$ & $\begin{array}{l}\text { mixed } \\
\text { (NA) }\end{array}$ & 7 & NA & medium & no \\
\hline $\begin{array}{l}\text { Oslund } \\
(2009)\end{array}$ & $\begin{array}{c}\text { ed, ex, bt, re, } \\
\text { sm }\end{array}$ & $\mathrm{X}$ & $\mathrm{X}$ & $\mathrm{X}$ & $\mathrm{X}$ & - & - & $\begin{array}{l}\text { mixed } \\
(\mathrm{NA})\end{array}$ & 4 & 120 & low & no \\
\hline $\begin{array}{l}\text { Persson } \\
\text { (2012) }\end{array}$ & $\begin{array}{c}\text { ed, ex, bt, re, } \\
\text { sm, ba, wo, } \\
\text { te, oth }\end{array}$ & $\mathrm{X}$ & $\mathrm{X}$ & $\mathrm{X}$ & $\mathrm{X}$ & $\mathrm{X}$ & $\mathrm{X}$ & $\begin{array}{l}\text { group } \\
(9)\end{array}$ & 5 & 126 & low & yes \\
\hline $\begin{array}{l}\text { Pietilä- } \\
\text { Holmner } \\
(2020)\end{array}$ & $\begin{array}{c}\text { ed, ex, bt, re, } \\
\text { sm }\end{array}$ & $\mathrm{X}$ & $\mathrm{X}$ & $\mathrm{X}$ & $\mathrm{X}$ & - & $\mathrm{X}$ & $\begin{array}{l}\text { mixed } \\
\text { (NA) }\end{array}$ & 8 & 20 & low & no \\
\hline $\begin{array}{l}\text { Reck } \\
(2017)\end{array}$ & $\begin{array}{c}\text { ed, ex, bt, re, } \\
\text { sm, wo }\end{array}$ & $\mathrm{X}$ & $\mathrm{X}$ & $\mathrm{X}$ & $\mathrm{X}$ & - & - & $\begin{array}{l}\text { group } \\
\text { (NA) }\end{array}$ & 1 & 45 & low & yes \\
\hline $\begin{array}{l}\text { Richardson } \\
\text { (1994) }\end{array}$ & $\begin{array}{l}\text { ed, ex, ga, bt, } \\
\text { re, sm, oth }\end{array}$ & - & $\mathrm{X}$ & $\mathrm{X}$ & $\mathrm{X}$ & $\mathrm{X}$ & - & $\begin{array}{l}\text { group } \\
(5)\end{array}$ & NA & 120 & low & no \\
\hline
\end{tabular}


Table 2. Continued.

\begin{tabular}{|c|c|c|c|c|c|c|c|c|c|c|c|c|}
\hline \multirow[b]{2}{*}{ Cohort } & \multirow[b]{2}{*}{$\begin{array}{l}\text { Treatment } \\
\text { modalities }\end{array}$} & \multicolumn{6}{|c|}{ Healthcare providers } & \multirow{2}{*}{$\begin{array}{c}\text { Type of } \\
\text { contact } \\
\text { (group size) }\end{array}$} & \multirow{2}{*}{$\begin{array}{l}\text { Time } \\
\text { span } \\
\text { (wks) }\end{array}$} & \multirow[b]{2}{*}{$\begin{array}{c}\text { Duration } \\
\text { (hours) }\end{array}$} & \multirow[b]{2}{*}{$\begin{array}{l}\text { Level of } \\
\text { tailoring }\end{array}$} & \multirow[b]{2}{*}{$\begin{array}{c}\text { follow } \\
\text { up? }\end{array}$} \\
\hline & & $\frac{\vec{a}}{a}$ & 启 & $\ddot{\mathrm{a}}$ & ప & $\mathbf{\Xi}$ & 总 & & & & & \\
\hline $\begin{array}{l}\text { Roche- } \\
\text { Leboucher } \\
(2011)\end{array}$ & $\begin{array}{c}\text { ex, re, wo, te, } \\
\text { oth }\end{array}$ & $\mathrm{X}$ & $\mathrm{X}$ & - & $\mathrm{X}$ & - & - & $\begin{array}{c}\text { group } \\
(6-8)\end{array}$ & 5 & 150 & low & no \\
\hline $\begin{array}{l}\text { Semrau } \\
(2015)\end{array}$ & $\begin{array}{c}\text { ed, ex, re, sm, } \\
\text { oth }\end{array}$ & $\mathrm{X}$ & $\mathrm{X}$ & $\mathrm{X}$ & - & - & $\mathrm{X}$ & $\begin{array}{l}\text { mixed } \\
\text { (NA) }\end{array}$ & 3 & 48 & low & no \\
\hline $\begin{array}{l}\text { Silvemark } \\
(2014)\end{array}$ & $\begin{array}{c}\text { ed, ex, bt, re, } \\
\text { sm, ba }\end{array}$ & $\mathrm{X}$ & $\mathrm{X}$ & $\mathrm{X}$ & $\mathrm{X}$ & $\mathrm{X}$ & $\mathrm{X}$ & $\begin{array}{c}\text { group } \\
(6-9)\end{array}$ & 5 & 175 & low & no \\
\hline $\begin{array}{l}\text { Smeets } \\
\text { (2008): a }\end{array}$ & ed, ga, bt, oth & $\mathrm{X}$ & $\mathrm{X}$ & $\mathrm{X}$ & $\mathrm{X}$ & - & $\mathrm{X}$ & group & 10 & 24 & low & no \\
\hline $\begin{array}{l}\text { Smeets } \\
(2008): \mathrm{b}\end{array}$ & $\begin{array}{c}\text { ed, ex, ga, bt, } \\
\text { oth }\end{array}$ & $\mathrm{X}$ & $\mathrm{X}$ & $\mathrm{X}$ & $\mathrm{X}$ & - & $\mathrm{X}$ & group & 10 & 77 & low & no \\
\hline $\begin{array}{l}\text { Spinhoven } \\
(2004)\end{array}$ & $\begin{array}{c}\text { ed, ga, bt, sm, } \\
\text { oth }\end{array}$ & - & $\mathrm{X}$ & $\mathrm{X}$ & $\mathrm{X}$ & - & - & mixed & 8 & 150 & low & no \\
\hline $\begin{array}{l}\text { Stein } \\
(2013)\end{array}$ & $\begin{array}{l}\text { ed, ex, bt, re, } \\
\text { sm, ba, te, oth }\end{array}$ & $\mathrm{X}$ & $\mathrm{X}$ & $\mathrm{X}$ & $\mathrm{X}$ & - & - & $\begin{array}{c}\text { group } \\
(6-8)\end{array}$ & 6 & 90 & low & no \\
\hline $\begin{array}{l}\text { Steinmetz } \\
\text { (2019) }\end{array}$ & ex, re, ph, oth & $\mathrm{X}$ & $\mathrm{X}$ & $\mathrm{X}$ & - & - & - & NA & 2 & 15 & high & no \\
\hline $\begin{array}{l}\text { Strobel } \\
(1998)\end{array}$ & $\begin{array}{c}\text { ed, ex, ga, re, } \\
\text { sm, ba, oth }\end{array}$ & $\mathrm{X}$ & $\mathrm{X}$ & $\mathrm{X}$ & - & $\mathrm{X}$ & - & $\begin{array}{l}\text { group } \\
(6)\end{array}$ & 5 & 150 & low & no \\
\hline $\begin{array}{l}\text { Tavafian } \\
(2011)\end{array}$ & $\begin{array}{c}\text { ed, ex, bt, re, } \\
\text { sm, ph, oth }\end{array}$ & - & $\mathrm{X}$ & $\mathrm{X}$ & - & - & - & $\begin{array}{l}\text { group } \\
\text { (NA) }\end{array}$ & 1 & 9 & low & yes \\
\hline $\begin{array}{l}\text { Thieme } \\
\text { (2003) }\end{array}$ & ed, ex, bt, sm & $\mathrm{X}$ & $\mathrm{X}$ & $\mathrm{X}$ & - & $\mathrm{X}$ & - & $\begin{array}{c}\text { group } \\
(5-7)\end{array}$ & 5 & 75 & low & no \\
\hline $\begin{array}{l}\text { Van der Maas } \\
\text { (2015): a }\end{array}$ & $\begin{array}{l}\text { ed, ex, ga, bt, } \\
\text { re, oth }\end{array}$ & $\mathrm{X}$ & $\mathrm{X}$ & $\mathrm{X}$ & $\mathrm{X}$ & - & - & $\begin{array}{c}\text { group } \\
(4-6)\end{array}$ & 12 & 94 & low & yes \\
\hline $\begin{array}{l}\text { Van der Maas } \\
(2015): \text { b }\end{array}$ & $\begin{array}{l}\text { ed, ex, ga, bt, } \\
\text { re, ba, oth }\end{array}$ & $\mathrm{X}$ & $\mathrm{X}$ & $\mathrm{X}$ & $\mathrm{X}$ & - & - & $\begin{array}{c}\text { group } \\
(4-6)\end{array}$ & 12 & 109 & low & yes \\
\hline $\begin{array}{l}\text { van Hooff } \\
(2010)\end{array}$ & $\begin{array}{c}\text { ed, ex, bt, re, } \\
\text { sm }\end{array}$ & - & $\mathrm{X}$ & $\mathrm{X}$ & $\mathrm{X}$ & - & - & $\begin{array}{l}\text { group } \\
\text { (NA) }\end{array}$ & 2 & 100 & low & no \\
\hline $\begin{array}{l}\text { van Wilgen } \\
\text { (2009) }\end{array}$ & $\begin{array}{l}\text { ed, ex, ga, bt, } \\
\text { re, sm, oth }\end{array}$ & $\mathrm{X}$ & $\mathrm{X}$ & $\mathrm{X}$ & - & - & - & $\begin{array}{l}\text { mixed } \\
(\mathrm{NA})\end{array}$ & 7 & NA & high & no \\
\hline $\begin{array}{l}\text { Vendrig } \\
(2000)\end{array}$ & $\begin{array}{c}\text { ed, ex, ga, ph, } \\
\text { wo }\end{array}$ & - & $\mathrm{X}$ & $\mathrm{X}$ & - & - & - & $\begin{array}{l}\text { group } \\
\text { (NA) }\end{array}$ & 4 & NA & low & no \\
\hline $\begin{array}{l}\text { Verkerk } \\
\text { (2011) }\end{array}$ & $\begin{array}{l}\text { ed, ex, re, } \\
\text { sm, ba }\end{array}$ & $\mathrm{X}$ & $\mathrm{X}$ & $\mathrm{X}$ & - & - & - & $\begin{array}{l}\text { group } \\
(6)\end{array}$ & 9 & 48 & low & yes \\
\hline $\begin{array}{l}\text { Volker } \\
(2017)\end{array}$ & ed, ex, bt, re & $\mathrm{X}$ & $\mathrm{X}$ & $\mathrm{X}$ & $\mathrm{X}$ & - & $\mathrm{X}$ & $\begin{array}{l}\text { mixed } \\
\text { (NA) }\end{array}$ & 15 & NA & low & no \\
\hline
\end{tabular}


Table 2. Continued.

\begin{tabular}{|c|c|c|c|c|c|c|c|c|c|c|c|c|}
\hline \multirow[b]{2}{*}{ Cohort } & \multirow[b]{2}{*}{$\begin{array}{l}\text { Treatment } \\
\text { modalities }\end{array}$} & \multicolumn{6}{|c|}{ Healthcare providers } & \multirow{2}{*}{$\begin{array}{c}\text { Type of } \\
\text { contact } \\
\text { (group size) }\end{array}$} & \multirow{2}{*}{$\begin{array}{l}\text { Time } \\
\text { span } \\
\text { (wks) }\end{array}$} & \multirow[b]{2}{*}{$\begin{array}{c}\text { Duration } \\
\text { (hours) }\end{array}$} & \multirow[b]{2}{*}{$\begin{array}{c}\text { Level of } \\
\text { tailoring }\end{array}$} & \multirow[b]{2}{*}{$\begin{array}{c}\text { follow } \\
\text { up? }\end{array}$} \\
\hline & & $\overrightarrow{\mathrm{e}}$ & 岺 & $\ddot{\mathrm{a}}$ & $\ddot{\circ}$ & $\exists$ & 离 & & & & & \\
\hline $\begin{array}{l}\text { Vowles } \\
(2011)\end{array}$ & $\begin{array}{c}\text { ed, ex, bt, re, } \\
\text { sm, ba }\end{array}$ & $\mathrm{X}$ & $\mathrm{X}$ & $\mathrm{X}$ & $\mathrm{X}$ & $\mathrm{X}$ & - & $\begin{array}{l}\text { group } \\
\text { (NA) }\end{array}$ & 3.5 & 114 & low & no \\
\hline $\begin{array}{l}\text { Wagner } \\
\text { (2011) }\end{array}$ & $\begin{array}{c}\text { ed, ex, bt, re, } \\
\text { sm }\end{array}$ & $\mathrm{X}$ & $\mathrm{X}$ & $\mathrm{X}$ & $\mathrm{X}$ & $\mathrm{X}$ & - & $\begin{array}{l}\text { mixed } \\
\text { (NA) }\end{array}$ & 6 & 123 & high & no \\
\hline $\begin{array}{l}\text { Westman } \\
\text { (2006) }\end{array}$ & $\begin{array}{c}\text { ed, ex, bt, re, } \\
\text { ba, oth }\end{array}$ & $\mathrm{X}$ & $\mathrm{X}$ & $\mathrm{X}$ & - & - & - & $\begin{array}{l}\text { group } \\
(8-10)\end{array}$ & 8 & 140 & low & yes \\
\hline $\begin{array}{l}\text { Zhuk } \\
\text { (2018) }\end{array}$ & $\begin{array}{c}\text { ed, ex, bt, re, } \\
\text { sm, ba, oth }\end{array}$ & $\mathrm{X}$ & $\mathrm{X}$ & $\mathrm{X}$ & - & $\mathrm{X}$ & - & $\begin{array}{l}\text { group } \\
\text { (NA) }\end{array}$ & 3 & NA & low & no \\
\hline
\end{tabular}

Notes: abbreviations: phy = physician; psy = psychologist pt = physiotherapist ot = occupational therapist nur $=$ nurse; swo = social worker; ed = education; $\mathrm{ex}=$ exercise; re = relaxation; $\mathrm{bt}=$ (cognitive) behavioral therapy; ga = graded activity; $\mathrm{ph}$ = pharmacological therapy; wo = workplace advice; te = team meetings; sm = self-management skills; ba = body awareness therapy; oth = other type of treatment modality. 


\section{Effect of Time}

For all outcomes, the median pre-post effect sizes show a favorable trend, indicating a positive change in health from pre to post treatment (range $=.38-1.94$ ). The post to finalfollow up effect sizes vary from -.49 to .15, indicating different trends. The median effect sizes from pre to final follow-up show an overall favorable change in health outcomes during the course of the study (range $=.32$ to .85). Table 3 shows the median effect size, range and the amount of statistical heterogeneity per contrast. The table also includes an overview of the number of cohorts that follow a particular pattern of effect over time, symbolized by different plotlines. For example, a statistically significant favorable pre-post effect that is followed by no effect from post to follow-up is represented by a positive slope, that flattens halfway.

The general trend across all outcomes indicates a statistically significant favorable effect of time in $85 \%$ of the pre-post effect sizes. This is reflected in a positive median effect size (median SMC $=.63$, range $=-.21$ to 4.93 ). Fifteen percent of the effect sizes shows no pre-post effects and there were no statistically significant unfavorable effects. For all cohorts that included a measurement at pre, post and follow-up time points, a pattern with a significant pre-post effect that is maintained at follow-up was found in 72 (49\%) of the cases. Twenty-three patterns (16\%) indicated a favorable pre-post effect that further improved at follow-up. A triangular relapse pattern was found in 29 (20\%) of the calculated effect sizes. Two patterns (1\%) showed no effect from pre to post, but a positive effect from post to follow-up and 17 outcomes (12\%) did not show any effect from pre to post or from post to follow-up. Three outcomes (2\%) showed no pre-post effect, but an unfavorable effect from post to follow-up. Finally, the dataset did not contain any pattern with statistically significant unfavorable pre-post results. Not all studies included a posttreatment measure, which explains why the pre to final follow-up evaluations include more effect sizes than in the pattern analysis. The effect of time from pre to final follow-up was favorable for 168 (77\%) of the effect sizes. Forty-seven effect sizes (21\%) did not indicate an effect and four effect sizes (2\%) showed an unfavorable effect over time. In the multimedia appendix, we provided time-series plots where we standardized each outcome measure on a scale from 0 to 100 (percentage of maximum score of the measurement instrument) and plotted the development of each cohort over time using all available data points. 


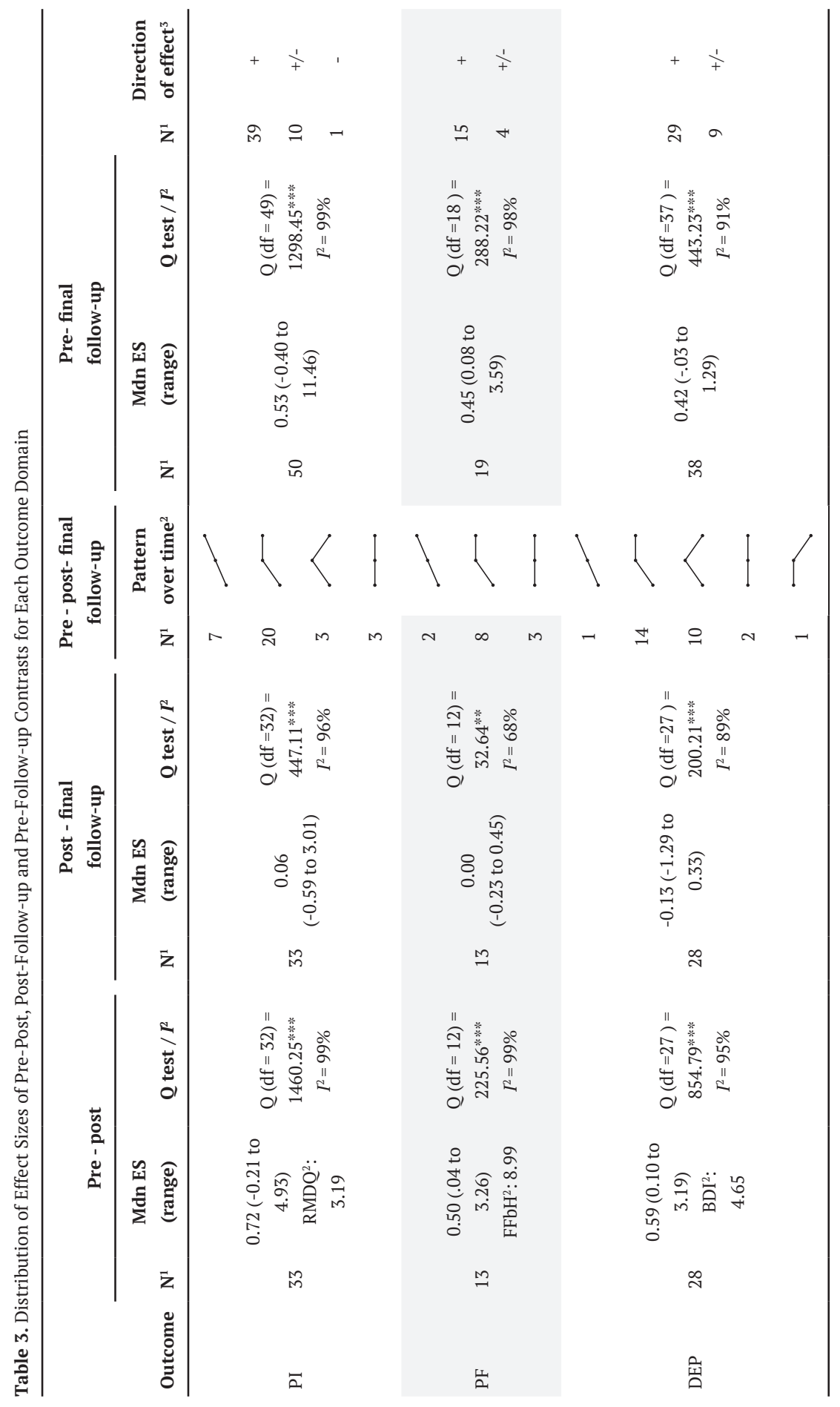




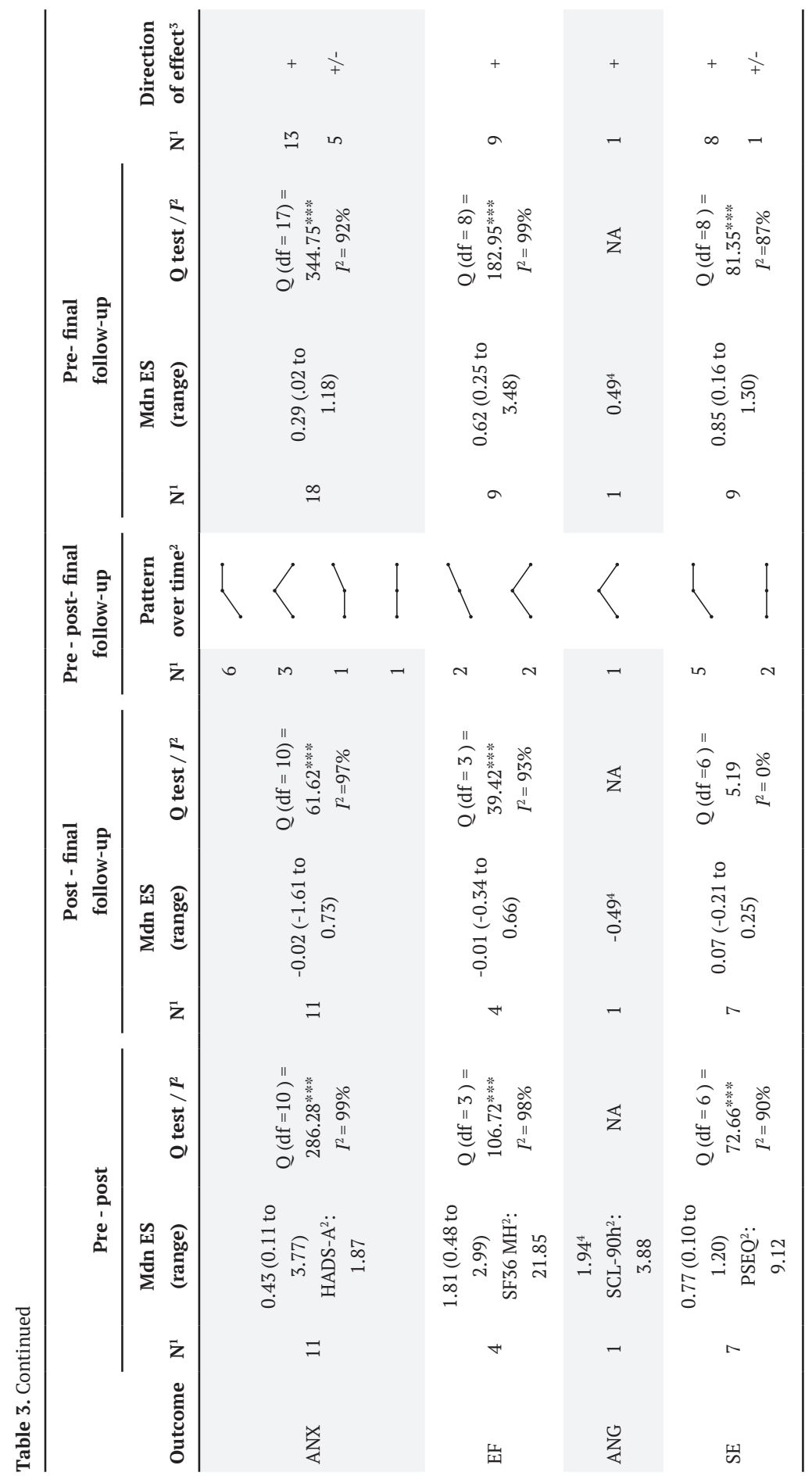




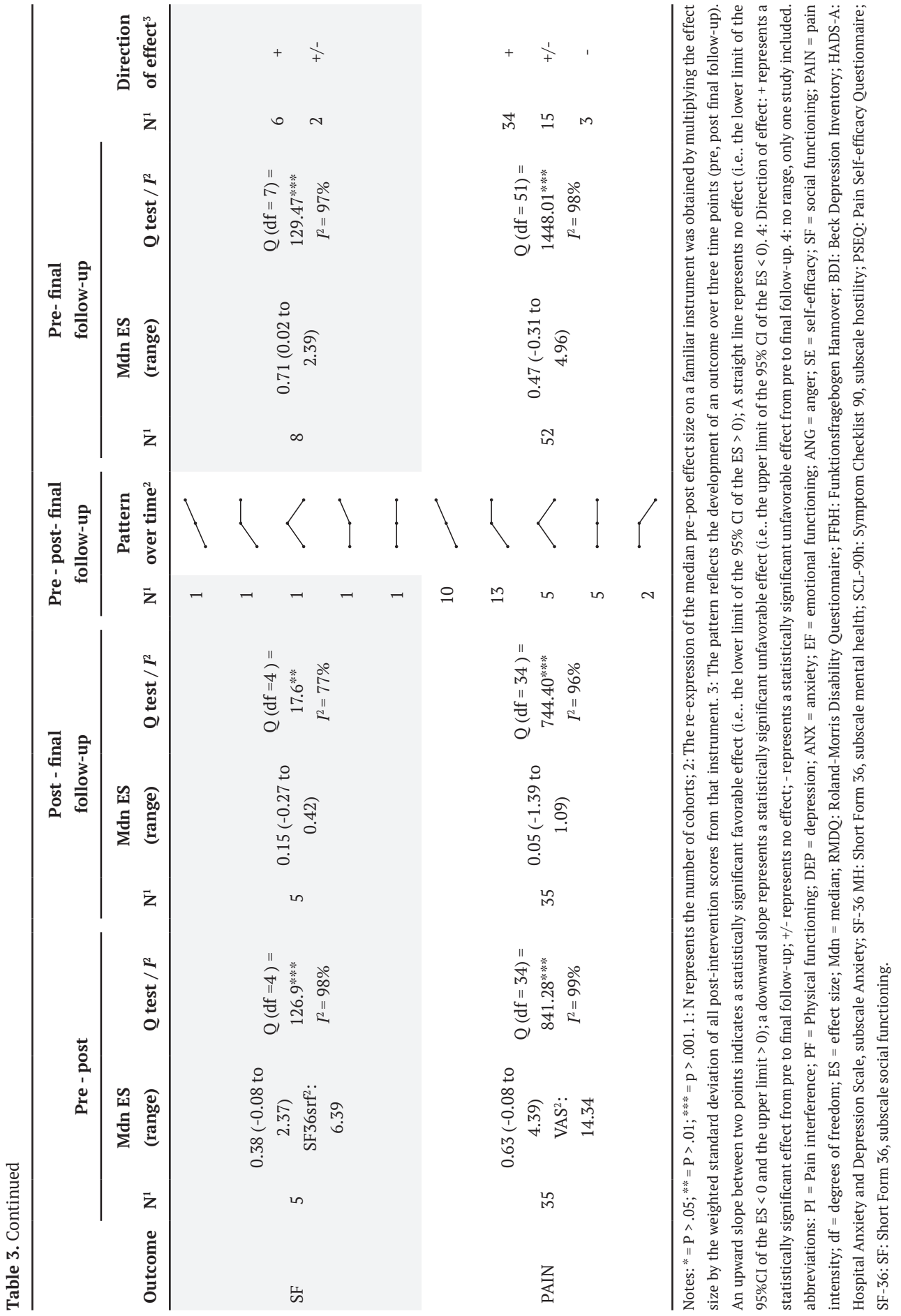


Heterogeneity in Outcomes. The Cochrane's Q-tests for all outcomes at each of the three contrasts were significant, except for self-efficacy at post-follow-up. For all other contrasts, this indicates that the null hypotheses that these studies are evaluating the same effect was rejected (Higgins \& Thompson, 2002). In addition, only except for self-efficacy at postfollow $\left(I^{2}=0 \%\right.$ ), all values were considerably high, with the majority of the values over $90 \%$. These analyses support our decision to refrain from pooling the effect sizes. Rather, multiple different patient, study or interventions factors may account for this variability. The self-efficacy post-follow-up contrasts indicate a stable maintenance pattern across studies.

\section{DISCUSSION}

\section{Summary of Findings}

Our first objective was to investigate the development over time of patients who participated in IMPT programmes. The results indicate that the majority of the patient cohorts significantly improved from pre to post-treatment. Importantly, this was mostly maintained at final follow-up, which is in contrast to typical triangular relapse patterns that have been observed in other health behaviour change efforts (Wood \& Neal, 2016). Although the results indicate that pre-post effects of IMPT are generally maintained over time, the possibility of relapse for individual patients should not be neglected. Closer inspection of the distribution of individual cohort data, such as the post to follow-up physical functioning data of Silvemark et al. (2014) (SMD $=0.06,95 \% \mathrm{CI}=-0.15$ to 0.28 ), reveals that $47 \%$ of the patients show a decrease in physical functioning, assuming normally distributed data and a pretest posttest correlation of $r=.59$. To increase the accuracy of these rudimentary estimates, publishing the datasets along with the study, would allow for more detailed analyses on patient relapse across studies. This is especially relevant when taking into account that IMPT programmes are often considered as treatment of last resort (Jeffery et al., 2011).

Our second objective was to explore the study, patient, intervention and outcome heterogeneity of the included cohorts. In line with our expectations and with previous studies, we observed substantial methodological and statistical heterogeneity despite overlapping theoretical foundations, such as the biopsychosocial model (Geneen et al., 2017; Guzmán et al., 2001; Waterschoot et al., 2014). This heterogeneity can be explained by different policies, cultures, resources, and research traditions that have been influencing these treatment programmes over time (Kaiser et al., 2018; Kaiser et al., 2017). However, to our knowledge the current study is the first attempt to extract and categorize the individual treatment modalities of IMPT programmes to assess the treatment content heterogeneity in 
these programmes. Despite a common heritage, the results of this assessment indicate that the included interventions do not share an equal underlying effect. Rather, interventions generally consist of a unique collection of multiple different action mechanisms that are generally not explicitly described.

\section{Strengths and Limitations}

We encountered several problems regarding interpretation of the study data. First, for the majority of the cohorts, we identified a risk of bias, which negatively influences the validity or our results. Especially the study attrition rates, indicating that a substantial minority dropped out of the programme or discontinued participation, introduce significant nonresponse bias. Furthermore, incomplete reporting of the intervention and its outcomes remains an issue. To increase accuracy of reporting as well as improved understanding of how a particular IMPT programme may benefit patients, we restate the recommendation by Williams and colleagues (2012) to provide a clear rationale for that particular set of treatment components and to test this by including process measures (e.g. Nicholas et al., 2014), instead of generally referring to a biopsychosocial approach. A practical tool that supports clear reporting is the TIDieR checklist, which includes clear guidance for reporting study rationale and action mechanisms (Hoffmann et al., 2014). Beyond the investigation of treatment benefit and harms of IMPT a standardized level of comparison, e.g. a core outcome set (Williamson et al., 2017), is required for harmonization of outcome assessment and supporting detailed meta analyses. Heterogeneous outcome assessment in the context of IMPT has been consistently reported (Deckert et al., 2016; Kamper et al., 2014; Waterschoot et al., 2014). Involving the patient perspective in defining helpful treatment approaches is generally recommended for such actions (Williamson et al., 2017). For IMPT an international initiative has developed a core outcome set for a domain set of outcomes, comprising the biopsychosocial impact of chronic pain in patients undergoing IMPT (Kaiser et al., 2018). Implementing such recommendations would help also to reduce reporting bias and enhance reporting quality of clinical trials (Williamson et al., 2017). On a smaller scale, successful implementation of similar initiatives has resulted in improved collaboration between healthcare services and a homogeneous dataset (Tardif et al., 2017). It should be noted that such initiatives are either shaped by national requirements and resources, commonly organized in national registries, or aim for international application in clinical trials (Kaiser et al., 2018). An important future challenge is to harmonize these approaches in order to achieve results in both objectives. Second, the categorization of treatment modalities is likely to contain erroneous interpretations, either due to incomplete reporting or to misinterpretation during the data extraction. Moreover, it is important to realize that the categories for the treatment modalities, the classification of tailoring and what constitutes as an IMPT programme remain arbitrary and leave room for discussion 
and further refinement in future studies. To provide transparency regarding our procedures and choices, we published all data extraction forms in the online multimedia appendix. Third, the calculation of pre-post effect sizes in meta-analyses is under debate (Cuijpers et al., 2017; Kösters, 2017). We realize that pre-post effects should not be considered as a valid method for demonstrating a treatment effect. However, the current analysis does provide an indication of the change over time of patients' wellbeing on several key outcomes, which is considered particularly useful from a clinician's perspective (Kösters, 2017). Of notice is that the suggested overestimation of effect size has not been observed in large comparison studies (Anglemyer et al., 2014; Benson \& Hartz, 2000; Concato et al., 2000). On the contrary, less heterogeneity was found of observational studies compared to RCTs. A possible explanation was that cohorts within case series potentially better represent the population at risk and tailor treatment to specific patients, compared to RCTs with specific inclusion criteria and standardized protocols.

\section{Future Directions}

An opportunity to increase the lifespan and relevance of this systematic review is to develop this study into a living systematic review. The main characteristic of this type of review is that it will be continuously updated when new evidence becomes available (Elliott et al., 2017). In addition, living systematic reviews often include an online platform where datasets and data analysis syntaxes are publicly available, which may decrease duplicate work (Thomas et al., 2017). This helps to decrease the evidence to practice gap, but also to stimulate collaboration and data-sharing (Elliott et al., 2014). Data validation by authors of the included studies, improved analyses techniques as well as semi-automated search and data extraction procedures are among the possibilities of such an initiative (BannachBrown et al., 2019; Thomas et al., 2017). The current multimedia appendix has been developed to accommodate future updates, which will facilitate this transition.

\section{Conclusions}

In the past five decades, pain management programmes evolved from attempts to coordinate various disciplines in managing chronic pain to comprehensive interdisciplinary multimodal interventions that help patients to optimize their daily life functioning and their overall wellbeing. This study shows that participation in an IMPT programme is associated with considerable improvements in physical and psychological wellbeing that are generally maintained at follow-up. The current study also revealed that despite common roots these programmes show substantial heterogeneity with respect to dose and treatment content, which suggests different viewpoints on how to optimally design an IMPT intervention. To discuss these differences and learn from this variability, we recommend to improve the precision of describing the intervention rationale and to test the proposed mechanisms by 
which the intervention is expected to benefit the patient. Finally, we believe that regular updates of this review may support the critical monitoring of future developments of IMPT programmes, the possibility to correct for data extraction errors and the comparison of different treatment approaches. A living systematic review approach provides the potential to accommodate this.

\section{Acknowledgments}

We greatly appreciate the help that we received from Myrthe Konijnenburg with the data extraction and the expertise of Dr. Marc Teunis, Julien Groenenboom, Mees Fröberg, and Britte Heijink for during the development and deployment of the online multimedia appendix.

\section{Author Contributions}

SE, UK, HW and RS conceived of the presented idea. JK and SE developed the search strategy. SE, SK, UK and JP screened the studies and extracted the data. HW and RS supervised the study. SE and SK developed the online multimedia appendix. All authors discussed the results and contributed to the final version of the manuscript. 


\section{REFERENCES}

Abbasi, M., Dehghani, M., Keefe, F. J., Jafari, H., Behtash, H., \& Shams, J. (2012). Spouse-assisted training in pain coping skills and the outcome of multidisciplinary pain management for chronic low back pain treatment: a 1-year randomized controlled trial. Eur J Pain, 16(7), 1033-1043. https://doi.org/10.1002/j.1532-2149.2011.00097.x

Anglemyer, A., Horvath, H. T., \& Bero, L. (2014). Healthcare outcomes assessed with observational study designs compared with those assessed in randomized trials. Cochrane Database Syst Rev(4), Mr000034. https://doi. org/10.1002/14651858.MR000034.pub2

Balk, E. M., Earley, A., Patel, K., Trikalinos, T. A., \& Dahabreh, I. J. (2012). AHRQ Methods for Effective Health Care. In Empirical Assessment of Within-Arm Correlation Imputation in Trials of Continuous Outcomes. Agency for Healthcare Research and Quality (US).

Bannach-Brown, A., Przybyła, P., Thomas, J., Rice, A. S. C., Ananiadou, S., Liao, J., \& Macleod, M. R. (2019). Machine learning algorithms for systematic review: reducing workload in a preclinical review of animal studies and reducing human screening error. Syst Rev, 8(1), 23. https://doi.org/10.1186/s13643-019-0942-7

Beaudreuil, J., Kone, H., Lasbleiz, S., Vicaut, E., Richette, P., Cohen-Solal, M., Lioté, F., de Vernejoul, M. C., Nizard, R., Yelnik, A., Bardin, T., \& Orcel, P. (2010). Efficacy of a functional restoration program for chronic low back pain: prospective 1-year study. Joint Bone Spine, 77(5), 435-439. https://doi.org/10.1016/j.jbspin.2010.03.003

Becker, B. J. (1988). Synthesizing standardized mean-change measures. Br J Math Stat Psychol, 41.2, 257-278.

Bendix, A. F., Bendix, T., Labriola, M., \& Boekgaard, P. (1998). Functional restoration for chronic low back pain. Two-year follow-up of two randomized clinical trials. Spine (Phila Pa 1976), 23(6), 717-725. https://doi. org/10.1097/00007632-199803150-00013

Bendix, T., Bendix, A., Labriola, M., Haestrup, C., \& Ebbehøj, N. (2000). Functional restoration versus outpatient physical training in chronic low back pain: a randomized comparative study. Spine (Phila Pa 1976), 25(19), 2494-2500. https://doi.org/10.1097/00007632-200010010-00012

Benson, K., \& Hartz, A. J. (2000). A comparison of observational studies and randomized, controlled trials. $N$ Engl J Med, 342(25), 1878-1886. https://doi.org/10.1056/nejm200006223422506

Bergström, G., Jensen, I. B., Bodin, L., Linton, S. J., \& Nygren, A. L. (2001). The impact of psychologically different patient groups on outcome after a vocational rehabilitation program for long-term spinal pain patients. Pain, 93(3), 229-237. https://doi.org/10.1016/s0304-3959(01)00320-7

Bergström, M., Ejelöv, M., Mattsson, M., \& Stålnacke, B. (2014). One-year follow-up of body awareness and perceived health after participating in a multimodal pain rehabilitation programme-a pilot study. Eur J Phys Ther, 16(4), 246-254.

Bileviciute-Ljungar, I., \& Norrefalk, J. R. (2014). Beneficial long-term effects of multiprofessional assessment vs. rehabilitation program in patients with musculoskeletal pain. Pain Pract, 14(3), 228-236. https://doi. org/10.1111/papr.12053

Borenstein, M., Higgins, J. P., Hedges, L. V., \& Rothstein, H. R. (2017). Basics of meta-analysis: I(2) is not an absolute measure of heterogeneity. Res Synth Methods, 8(1), 5-18. https://doi.org/10.1002/jrsm.1230

Borys, C., Lutz, J., Strauss, B., \& Altmann, U. (2015). Effectiveness of a Multimodal Therapy for Patients with Chronic Low Back Pain Regarding Pre-Admission Healthcare Utilization. PloS One, 10(11), e0143139. https://doi. org/10.1371/journal.pone.0143139

Brendbekken, R., Harris, A., Ursin, H., Eriksen, H. R., \& Tangen, T. (2016). Multidisciplinary Intervention in Patients with Musculoskeletal Pain: a Randomized Clinical Trial. Int J Behav Med, 23(1), 1-11. https://doi.org/10.1007/ s12529-015-9486-y 
Brouwer, M. E., Williams, A. D., Kennis, M., Fu, Z., Klein, N. S., Cuijpers, P., \& Bockting, C. L. H. (2019). Psychological theories of depressive relapse and recurrence: A systematic review and meta-analysis of prospective studies. Clin Psychol Rev, 74, 101773. https://doi.org/10.1016/j.cpr.2019.101773

Cardosa, M., Osman, Z. J., Nicholas, M., Tonkin, L., Williams, A., Abd Aziz, K., Mohd Ali, R., \& Dahari, N. M. (2012). Self-management of chronic pain in Malaysian patients: effectiveness trial with 1-year follow-up. Transl Behav Med, 2(1), 30-37. https://doi.org/10.1007/s13142-011-0095-2

Carver, C. S., \& Scheier, M. F. (2017). Self-regulatory functions supporting motivated action, Advan Mot Sci, 4, 1-37. https://doi.org/10.1016/bs.adms.2017.02.002

Clond, M. (2016). Emotional freedom techniques for anxiety: A systematic review with meta-analysis. J Nerv Ment Dis, 204(5), 388-395. https://doi.org/10.1097/nmd.0000000000000483

Concato, J., Shah, N., \& Horwitz, R. I. (2000). Randomized, controlled trials, observational studies, and the hierarchy of research designs. N Engl J Med, 342(25), 1887-1892. https://doi.org/10.1056/nejm200006223422507

Cuijpers, P., Weitz, E., Cristea, I. A., \& Twisk, J. (2017). Pre-post effect sizes should be avoided in meta-analyses. Epidemiol Psychiatr Sci, 26(4), 364-368. https://doi.org/10.1017/s2045796016000809

de Rooij, A., de Boer, M. R., van der Leeden, M., Roorda, L. D., Steultjens, M. P., \& Dekker, J. (2014). Cognitive mechanisms of change in multidisciplinary treatment of patients with chronic widespread pain: a prospective cohort study. J Rehabil Med, 46(2), 173-180. https://doi.org/10.2340/16501977-1252

Deckert, S., Kaiser, U., Kopkow, C., Trautmann, F., Sabatowski, R., \& Schmitt, J. (2016). A systematic review of the outcomes reported in multimodal pain therapy for chronic pain. Eur J Pain, 20(1), 51-63. https://doi. org/10.1002/ejp.721

Dworkin, R. H., Turk, D. C., Farrar, J. T., Haythornthwaite, J. A., Jensen, M. P., Katz, N. P., Kerns, R. D., Stucki, G., Allen, R. R., Bellamy, N., Carr, D. B., Chandler, J., Cowan, P., Dionne, R., Galer, B. S., Hertz, S., Jadad, A. R., Kramer, L. D., Manning, D. C., Martin, S., McCormick, C. G., McDermott, M. P., McGrath, P., Quessy, S., Rappaport, B. A., Robbins, W., Robinson, J. P., Rothman, M., Royal, M. A., Simon, L., Stauffer, J. W., Stein, W., Tollett, J., Wernicke, J., \& Witter, J. (2005). Core outcome measures for chronic pain clinical trials: IMMPACT recommendations. Pain, 113(1-2), 9-19. https://doi.org/10.1016/j.pain.2004.09.012

Dysvik, E., Kvaløy, J. T., \& Furnes, B. (2013). Evaluating physical functioning as part of a Cognitive Behavioural Therapy approach in treatment of people suffering from chronic pain. J Clin Nurs, 22(5-6), 806-816. https:// doi.org/10.1111/j.1365-2702.2011.04040.x

Elliott, J. H., Synnot, A., Turner, T., Simmonds, M., Akl, E. A., McDonald, S., Salanti, G., Meerpohl, J., MacLehose, H., Hilton, J., Tovey, D., Shemilt, I., \& Thomas, J. (2017). Living systematic review: 1. Introduction-the why, what, when, and how. J Clin Epidemiol, 91, 23-30. https://doi.org/10.1016/j.jclinepi.2017.08.010

Elliott, J. H., Turner, T., Clavisi, O., Thomas, J., Higgins, J. P., Mavergames, C., \& Gruen, R. L. (2014). Living systematic reviews: an emerging opportunity to narrow the evidence-practice gap. PLoS Med, 11(2), e1001603. https://doi. org/10.1371/journal.pmed.1001603

Frost, H., Lamb, S., \& Shackleton, C. J. P. (2000). A functional restoration programme for chronic low back pain: a prospective outcome study. Physiotherapy, 86(6), 285-293.

Gantschnig, B. E., Heigl, F., Widmer Leu, C., Bütikofer, L., Reichenbach, S., \& Villiger, P. M. (2017). Effectiveness of the Bern Ambulatory Interprofessional Rehabilitation (BAI-Reha) programme for patients with chronic musculoskeletal pain: a cohort study. Swiss Med Wkly, 147, w14433. https://doi.org/10.4414/smw.2017.14433

Gatchel, R. J., McGeary, D. D., McGeary, C. A., \& Lippe, B. (2014). Interdisciplinary chronic pain management: past, present, and future. Am Psychol, 69(2), 119-130. https://doi.org/10.1037/a0035514

Gatzounis, R., Schrooten, M. G., Crombez, G., \& Vlaeyen, J. W. (2012). Operant learning theory in pain and chronic pain rehabilitation. Curr Pain Headache Rep, 16(2), 117-126. https://doi.org/10.1007/s11916-012-0247-1 
Geneen, L. J., Moore, R. A., Clarke, C., Martin, D., Colvin, L. A., \& Smith, B. H. (2017). Physical activity and exercise for chronic pain in adults: an overview of Cochrane Reviews. Cochrane Database Syst Rev, 4(4), Cd011279. https:// doi.org/10.1002/14651858.CD011279.pub3

Gerdle, B., Molander, P., Stenberg, G., Stalnacke, B. M., \& Enthoven, P. (2016). Weak outcome predictors of multimodal rehabilitation at one-year follow-up in patients with chronic pain-a practice based evidence study from two SQRP centres. BMC Musculoskeletal Disord 17(1), 490. https://doi.org/10.1186/s12891-016-1346-7

Grahn, B., Ekdahl, C., \& Borgquist, L. (2000). Motivation as a predictor of changes in quality of life and working ability in multidisciplinary rehabilitation. A two-year follow-up of a prospective controlled study in patients with prolonged musculoskeletal disorders. Disabil Rehabil, 22(15), 639-654. https://doi. org/10.1080/096382800445443

Gustafsson, M., Ekholm, J., \& Broman, L. (2002). Effects of a multiprofessional rehabilitation programme for patients with fibromyalgia syndrome. J Rehabil Med, 34(3), 119-127. https://doi.org/10.1080/165019702753714147

Guzmán, J., Esmail, R., Karjalainen, K., Malmivaara, A., Irvin, E., \& Bombardier, C. (2001). Multidisciplinary rehabilitation for chronic low back pain: systematic review. BMJ, 322(7301), 1511-1516. https://doi.org/10.1136/ bmj.322.7301.1511

Hafenbrack, K., Heinrich, M., Müller, G., Marnitz, U., Mallwitz, J., \& Klinger, R. (2013). [Effects of interdisciplinary functional restoration treatment with cognitive behavior therapy in patients with chronic back pain: healthcare research in the context of selective contracts]. Schmerz, 27(6), 566-576. https://doi.org/10.1007/ s00482-013-1377-z (Effekte eines interdisziplinären Functional-restoration-Behandlungsprogramms mit kognitiv-behavioraler Therapie beim chronischen Rückenschmerz : Versorgungsforschung im Kontext von Selektivverträgen.)

Haiduk, P., Benz, T., Lehmann, S., Gysi-Klaus, F., Aeschlimann, A., Michel, B. A., \& Angst, F. (2017). Interdisciplinary rehabilitation after whiplash injury: An observational prospective 5 years outcome study. Medicine, 96(9), e6113. https://doi.org/10.1097/md.0000000000006113

Hållstam, A., Löfgren, M., Svensén, C., \& Stålnacke, B. M. (2016). Patients with chronic pain: One-year follow-up of a multimodal rehabilitation programme at a pain clinic. Scand J Pain, 10, 36-42. https://doi.org/10.1016/j. sjpain.2015.08.008

Hazard, R. G., Fenwick, J. W., Kalisch, S. M., Redmond, J., Reeves, V., Reid, S., \& Frymoyer, J. W. (1989). Functional restoration with behavioral support. A one-year prospective study of patients with chronic low-back pain. Spine (Phila Pa 1976), 14(2), 157-161.

Higgins, J. P., \& Thompson, S. G. (2002). Quantifying heterogeneity in a meta-analysis. Stat Med, 21(11), 1539-1558. https://doi.org/10.1002/sim.1186

Higgins, J. P. T., Chandler, J., Cumpston, M., Li, T., Page, M. J., \& Welch, V. A. (2019). Cochrane Handbook for Systematic Reviews of Interventions version 6.0 (updated July 2019). Cochrane. Available from: www.training.cochrane.org/ handbook.

Hildebrandt, J., Pfingsten, M., Franz, C., Saur, P., \& Seeger, D. (1996). [Multidisciplinary treatment program for chronic low back pain, part 1. Overview]. Schmerz, 10(4), 190-203. https://doi.org/10.1007/s004829600018 (Das Göttinger Rücken Intensiv Programm (GRIP)--ein multimodales Behandlungsprogramm für Patienten mit chronischen Rückenschmerzen, Teil 1 Ergebnisse im Uberblick.)

Hoffmann, T. C., Glasziou, P. P., Boutron, I., Milne, R., Perera, R., Moher, D., Altman, D. G., Barbour, V., Macdonald, H., Johnston, M., Lamb, S. E., Dixon-Woods, M., McCulloch, P., Wyatt, J. C., Chan, A. W., \& Michie, S. (2014). Better reporting of interventions: template for intervention description and replication (TIDieR) checklist and guide. BMJ, 348, g1687. https://doi.org/10.1136/bmj.g1687

Hozo, S. P., Djulbegovic, B., \& Hozo, I. (2005). Estimating the mean and variance from the median, range, and the size of a sample. BMC Med Res Methodol, 5, 13. https://doi.org/10.1186/1471-2288-5-13 
Huedo-Medina, T. B., Sánchez-Meca, J., Marín-Martínez, F., \& Botella, J. (2006). Assessing heterogeneity in metaanalysis: Q statistic or I2 index? Psychol Methods, 11(2), 193-206. https://doi.org/10.1037/1082-989x.11.2.193

Huffman, K. L., Mandell, D., Lehmann, J. K., Jimenez, X. F., \& Lapin, B. R. (2019). Clinical and Demographic Predictors of Interdisciplinary Chronic Pain Rehabilitation Program Treatment Response. J Pain, 20(12), 1470-1485. https://doi.org/10.1016/j.jpain.2019.05.014

Ibrahim, M. E., Weber, K., Courvoisier, D. S., \& Genevay, S. (2019). Recovering the capability to work among patients with chronic low Back pain after a four-week, multidisciplinary biopsychosocial rehabilitation program: 18-month follow-up study. BMC Musculoskelet Disord, 20(1), 439. https://doi.org/10.1186/s12891-019-2831-6

Jeffery MM, Butler M, Stark A, Kane RL. Multidisciplinary Pain Programs for Chronic Noncancer Pain. In: Multidisciplinary Pain Programs for Chronic Noncancer Pain. Agency for Healthcare Research and Quality (US), Rockville (MD); 2011. Available from https://www.ncbi.nlm.nih.gov/books/NBK82511 PMID: 22191120.

Jensen, I. B., Dahlquist, C., Nygren, Å., Royen, E., \& Stenberg, M. J. J. (1997). Treatment for “helpless” women suffering from chronic spinal pain: a randomized controlled 18-month follow-up study. J Occup Rehabil, 7(4), 225-238.

Kääpä, E. H., Frantsi, K., Sarna, S., \& Malmivaara, A. (2006). Multidisciplinary group rehabilitation versus individual physiotherapy for chronic nonspecific low back pain: a randomized trial. Spine (Phila Pa 1976), 31(4), 371-376. https://doi.org/10.1097/01.brs.0000200104.90759.8c

Kaiser, U., Kopkow, C., Deckert, S., Neustadt, K., Jacobi, L., Cameron, P., De Angelis, V., Apfelbacher, C., Arnold, B., Birch, J., Bjarnegård, A., Christiansen, S., A, C. d. C. W., Gossrau, G., Heinks, A., Hüppe, M., Kiers, H., Kleinert, U., Martelletti, P., McCracken, L., de Meij, N., Nagel, B., Nijs, J., Norda, H., Singh, J. A., Spengler, E., Terwee, C. B., Tugwell, P., Vlaeyen, J. W. S., Wandrey, H., Neugebauer, E., Sabatowski, R., \& Schmitt, J. (2018). Developing a core outcome domain set to assessing effectiveness of interdisciplinary multimodal pain therapy: the VAPAIN consensus statement on core outcome domains. Pain, 159(4), 673-683. https://doi.org/10.1097/j. pain.0000000000001129

Kaiser, U., Treede, R. D., \& Sabatowski, R. (2017). Multimodal pain therapy in chronic noncancer pain-gold standard or need for further clarification? Pain, 158(10), 1853-1859. https://doi.org/10.1097/j.pain.0000000000000902

Kamper, S. J., Apeldoorn, A. T., Chiarotto, A., Smeets, R. J., Ostelo, R. W., Guzman, J., \& van Tulder, M. W. (2014). Multidisciplinary biopsychosocial rehabilitation for chronic low back pain. Cochrane Database Syst Rev(9), Cd000963. https://doi.org/10.1002/14651858.CD000963.pub3

Koopman, F. S., Edelaar, M., Slikker, R., Reynders, K., van der Woude, L. H., \& Hoozemans, M. J. (2004). Effectiveness of a multidisciplinary occupational training program for chronic low back pain: a prospective cohort study. $A m$ J Phys Med Rehabil, 83(2), 94-103. https://doi.org/10.1097/01.Phm.0000107482.35803.11

Kösters, M. (2017). Every effect size has its place: a commentary on the avoidance of pre-post effect sizes. Epidemiol Psychiatr Sci, 26(4), 369-370. https://doi.org/10.1017/s204579601700004x

Lemstra, M., \& Olszynski, W. P. (2005). The effectiveness of multidisciplinary rehabilitation in the treatment of fibromyalgia: a randomized controlled trial. Clin J Pain, 21(2), 166-174. https://doi.org/10.1097/00002508200503000-00008

Letzel, J., Angst, F., \& Weigl, M. B. (2019). Multidisciplinary biopsychosocial rehabilitation in chronic neck pain: a naturalistic prospective cohort study with intraindividual control of effects and 12-month follow-up. Eur JPhys Rehabil Med, 55(5), 665-675. https://doi.org/10.23736/s1973-9087.18.05348-0

Lipsey, M. W., \& Wilson, D. B. (2001). Practical meta-analysis. Sage Publications.

Mangels, M., Schwarz, S., Worringen, U., Holme, M., \& Rief, W. (2009). Evaluation of a behavioral-medical inpatient rehabilitation treatment including booster sessions: a randomized controlled study. Clin J Pain, 25(5), 356-364. https://doi.org/10.1097/AJP.0b013e3181925791 
Martín, J., Torre, F., Padierna, A., Aguirre, U., González, N., García, S., Matellanes, B., \& Quintana, J. M. (2012). Six-and 12-month follow-up of an interdisciplinary fibromyalgia treatment programme: results of a randomised trial. Clin Exp Rheumatol, 30(6 Suppl 74), 103-111.

McAllister, M. J., McKenzie, K. E., Schultz, D. M., \& Epshteyn, M. G. (2005). Effectiveness of a multidisciplinary chronic pain program for treatment of refractory patients with complicated chronic pain syndromes. Pain Physician, 8(4), 369-373.

Merrick, D., \& Sjölund, B. H. (2009). Patients' pretreatment beliefs about recovery influence outcome of a pain rehabilitation program. Eur J Phys Rehabil Med, 45(3), 391-401.

Merrick, D., Sundelin, G., \& Stalnacke, B. M. (2012). One-year follow-up of two different rehabilitation strategies for patients with chronic pain. J Rehabil Med, 44(9), 764-773.

Meng, K., Seekatz, B., Roband, H., Worringen, U., Vogel, H., \& Faller, H. (2011). Intermediate and long-term effects of a standardized back school for inpatient orthopedic rehabilitation on illness knowledge and self-management behaviors: a randomized controlled trial \%J Clinical Journal of Pain. Clin J Pain, 27(3), 248-257.

Moher, D., Liberati, A., Tetzlaff, J., \& Altman, D. G. (2009). Preferred reporting items for systematic reviews and metaanalyses: the PRISMA statement. PLoS Med, 6(7), e1000097. https://doi.org/10.1371/journal.pmed.1000097

Moher, D., Shamseer, L., Clarke, M., Ghersi, D., Liberati, A., Petticrew, M., Shekelle, P., \& Stewart, L. A. (2015). Preferred reporting items for systematic review and meta-analysis protocols (PRISMA-P) 2015 statement. Syst Rev, 4(1), 1. https://doi.org/10.1186/2046-4053-4-1

Monticone, M., Ambrosini, E., Rocca, B., Cazzaniga, D., Liquori, V., \& Foti, C. (2016). Group-based task-oriented exercises aimed at managing kinesiophobia improved disability in chronic low back pain. Eur J Pain, 20(4), 541-551. https://doi.org/10.1002/ejp.756

Monticone, M., Ferrante, S., Rocca, B., Baiardi, P., Farra, F. D., \& Foti, C. (2013). Effect of a long-lasting multidisciplinary program on disability and fear-avoidance behaviors in patients with chronic low back pain: results of a randomized controlled trial. Clin J Pain, 29(11), 929-938. https://doi.org/10.1097/AJP.0b013e31827fef7e

Moola, S., Munn, Z., Tufanaru, C., Aromataris, E., Sears, K., Sfetcu, R., Currie, M., Qureshi, R., Mattis, P., Lisy, K., \& Mu, P.-F. (2017). Chapter 7: Systematic reviews of etiology and risk. In E. Aromataris \& Z. Munn (Eds.), Joanna Briggs Institute Reviewer's Manual. The Joanna Briggs Institute.

Morley, S. (2008). Relapse prevention: still neglected after all these years. Pain, 134(3), 239-240. https://doi. org/10.1016/j.pain.2007.12.004

Morris, S. B. (2000). Distribution of the standardized mean change effect size for meta-analysis on repeated measures. Br J Math Stat Psychol, 53 ( Pt 1), 17-29. https://doi.org/10.1348/000711000159150

Nagel, B., \& Korb, J. J.D. O. (2009). Multimodale therapie des rückenschmerzes: Nachhaltig wirksam und kosteneffectiv. Orthopäde, 38(10), 907-912. https://doi.org/1 0.1007/s00132-009-1482-y

Nicholas, M. K., Asghari, A., Sharpe, L., Beeston, L., Brooker, C., Glare, P., Martin, R., Molloy, A., \& Wrigley, P. J. (2020). Reducing the use of opioids by patients with chronic pain: an effectiveness study with long-term follow-up. Pain, 161(3), 509-519. https://doi.org/10.1097/j.pain.0000000000001763

Nicholas, M. K., Asghari, A., Sharpe, L., Brnabic, A., Wood, B. M., Overton, S., Tonkin, L., de Sousa, M., Finniss, D., Beeston, L., Sutherland, A., Corbett, M., \& Brooker, C. (2014). Cognitive exposure versus avoidance in patients with chronic pain: adherence matters. Eur J Pain, 18(3), 424-437. https://doi.org/10.1002/j.15322149.2013.00383.x

Olason, M. (2004). Outcome of an interdisciplinary pain management program in a rehabilitation clinic. Work, 22(1), 9-15.

Opozda, M., Chur-Hansen, A., \& Wittert, G. (2016). Changes in problematic and disordered eating after gastric bypass, adjustable gastric banding and vertical sleeve gastrectomy: a systematic review of pre-post studies. Obes Rev, 17(8), 770-792. https://doi.org/10.1111/obr.12425 
Oslund, S., Robinson, R. C., Clark, T. C., Garofalo, J. P., Behnk, P., Walker, B., Walker, K. E., Gatchel, R. J., Mahaney, M., \& Noe, C. E. (2009). Long-term effectiveness of a comprehensive pain management program: strengthening the case for interdisciplinary care. Proc (Bayl Univ Med Cent), 22(3), 211-214. https://doi.org/10.1080/0899828 0.2009 .11928516

Ouzzani, M., Hammady, H., Fedorowicz, Z., \& Elmagarmid, A. (2016). Rayyan-a web and mobile app for systematic reviews. Syst Rev, 5(1), 210. https://doi.org/10.1186/s13643-016-0384-4

Penney, L. S., \& Haro, E. (2019). Qualitative evaluation of an interdisciplinary chronic pain intervention: outcomes and barriers and facilitators to ongoing pain management. J Pain Res, 12, 865-878. https://doi.org/10.2147/jpr. S185652

Persson, E., Lexell, J., Eklund, M., \& Rivano-Fischer, M. (2012). Positive effects of a musculoskeletal pain rehabilitation program regardless of pain duration or diagnosis. $P M R, 4(5), 355-366$. https://doi.org/10.1016/j. pmrj.2011.11.007

Pietilä-Holmner, E., Enthoven, P., Gerdle, B., Molander, P., \& Stålnacke, B. M. (2020). Long-term outcomes of multimodal rehabilitation in primary care for patients with chronic pain. J Rehabil Med, 52(2), jrm00023. https://doi.org/10.2340/16501977-2649

R Core Team. (2013). R: A language and environment for statistical computing. R Foundation for Statistical Computing. http://www.R-project.org/

Reck, T., Dumat, W., Krebs, J., \& Ljutow, A. (2017). [Outpatient multimodal pain therapy : Results of a 1-week intensive outpatient multimodal group program for patients with chronic unspecific back pain - retrospective evaluation after 3 and 12 months]. Schmerz, 31(5), 508-515. https://doi.org/10.1007/s00482-017-0211-4 (Ambulante multimodale Schmerztherapie : Ergebnisse eines 1-wöchigen ambulanten intensiven multimodalen Gruppenprogramms für Patienten mit chronischen unspezifischen Rückenschmerzen - retrospektive Evaluation nach 3 und 12 Monaten.)

Richardson, I. H., Richardson, P. H., Williams, A. C., Featherstone, J., \& Harding, V. R. (1994). The effects of a cognitivebehavioral pain management programme on the quality of work and employment status of severely impaired chronic pain patients. Disabil Rehabil, 16(1), 26-34. https://doi.org/10.3109/09638289409166433

Roberts, B. W., Luo, J., Briley, D. A., Chow, P. I., Su, R., \& Hill, P. L. (2017). A systematic review of personality trait change through intervention. Psychol Bull, 143(2), 117-141. https://doi.org/10.1037/bul0000088

Roche-Leboucher, G., Petit-Lemanac'h, A., Bontoux, L., Dubus-Bausière, V., Parot-Shinkel, E., Fanello, S., PenneauFontbonne, D., Fouquet, N., Legrand, E., Roquelaure, Y., \& Richard, I. (2011). Multidisciplinary intensive functional restoration versus outpatient active physiotherapy in chronic low back pain: a randomized controlled trial. Spine (Phila Pa 1976), 36(26), 2235-2242. https://doi.org/10.1097/BRS.0b013e3182191e13

RStudio Team. (2020). RStudio: Integrated Development for R. In RStudio, PBC. http://www.rstudio.com/

Scascighini, L., Toma, V., Dober-Spielmann, S., \& Sprott, H. (2008). Multidisciplinary treatment for chronic pain: a systematic review of interventions and outcomes. Rheumatology (Oxford), 47(5), 670-678. https://doi. org/10.1093/rheumatology/ken021

Semrau, J., Hentschke, C., Buchmann, J., Meng, K., Vogel, H., Faller, H., Bork, H., \& Pfeifer, K. (2015). Long-term effects of interprofessional biopsychosocial rehabilitation for adults with chronic non-specific low back pain: a multicentre, quasi-experimental study. PloS One, 10(3), e0118609. https://doi.org/10.1371/journal. pone.0118609

Silvemark, A., Källmén, H., \& Molander, C. (2014). Improved life satisfaction and pain reduction: follow-up of a 5-week multidisciplinary long-term pain rehabilitation programme. Ups J Med Sci, 119(3), 278-286. https://doi. org/10.3109/03009734.2014.908252 
Smeets, R. J., Vlaeyen, J. W., Hidding, A., Kester, A. D., van der Heijden, G. J., \& Knottnerus, J. A. (2008). Chronic low back pain: physical training, graded activity with problem solving training, or both? The one-year post-treatment results of a randomized controlled trial. Pain, 134(3), 263-276. https://doi.org/10.1016/j.pain.2007.04.021

Spinhoven, P., Ter Kuile, M., Kole-Snijders, A. M., Hutten Mansfeld, M., Den Ouden, D. J., \& Vlaeyen, J. W. (2004). Catastrophizing and internal pain control as mediators of outcome in the multidisciplinary treatment of chronic low back pain. Eur J Pain, 8(3), 211-219. https://doi.org/10.1016/j.ejpain.2003.08.003

Stein, K. F., \& Miclescu, A. (2013). Effectiveness of multidisciplinary rehabilitation treatment for patients with chronic pain in a primary health care unit. Scand J Pain, 4(4), 190-197. https://doi.org/10.1016/j.sjpain.2013.06.003

Steinmetz, A., Psczolla, M., Seidel, W., Niemier, K., Derlien, S., \& Nisser, J. (2019). Effect of subgroup-specific multimodal therapy on chronic spinal back pain and function-a prospective inpatient multicentre clinical trial in Germany. Medicine, 98(1), e13825. https://doi.org/10.1097/md.0000000000013825

Strobel, E. S., Wild, J., \& Müller, W. (1998). [Interdisciplinary group therapy for fibromyalgia]. Z Rheumatol, 57(2), 8994. https://doi.org/10.1007/s003930050065 (Interdisziplinäre Gruppentherapie für die Fibromyalgie.)

Tardif, H., Arnold, C., Hayes, C., \& Eagar, K. (2017). Establishment of the Australasian electronic persistent pain outcomes collaboration. Pain Med, 18(6), 1007-1018.

Tavafian, S. S., Jamshidi, A. R., \& Mohammad, K. (2011). Treatment of chronic low back pain: a randomized clinical trial comparing multidisciplinary group-based rehabilitation program and oral drug treatment with oral drug treatment alone. Clin J Pain, 27(9), 811-818. https://doi.org/10.1097/AJP.0b013e31821e7930

Thieme, K., Gromnica-Ihle, E., \& Flor, H. (2003). Operant behavioral treatment of fibromyalgia: a controlled study. Arthritis Rheum, 49(3), 314-320. https://doi.org/10.1002/art.11124

Thomas, J., Noel-Storr, A., Marshall, I., Wallace, B., McDonald, S., Mavergames, C., Glasziou, P., Shemilt, I., Synnot, A., Turner, T., \& Elliott, J. (2017). Living systematic reviews: 2. Combining human and machine effort. J Clin Epidemiol, 91, 31-37. https://doi.org/10.1016/j.jclinepi.2017.08.011

Treede, R. D., Rief, W., Barke, A., Aziz, Q., Bennett, M. I., Benoliel, R., Cohen, M., Evers, S., Finnerup, N. B., First, M. B., Giamberardino, M. A., Kaasa, S., Kosek, E., Lavand'homme, P., Nicholas, M., Perrot, S., Scholz, J., Schug, S., Smith, B. H., Svensson, P., Vlaeyen, J. W., \& Wang, S. J. (2015). A classification of chronic pain for ICD-11. Pain, 156(6), 1003-1007. https://doi.org/10.1097/j.pain.0000000000000160

Turk, D. C. (2003). Cognitive-behavioral approach to the treatment of chronic pain patients. Reg Anesth Pain Med, 28(6), 573-579. https://doi.org/10.1016/s1098-7339(03)00392-4

Turk, D. C., Dworkin, R. H., Allen, R. R., Bellamy, N., Brandenburg, N., Carr, D. B., Cleeland, C., Dionne, R., Farrar, J. T., Galer, B. S., Hewitt, D. J., Jadad, A. R., Katz, N. P., Kramer, L. D., Manning, D. C., McCormick, C. G., McDermott, M. P., McGrath, P., Quessy, S., Rappaport, B. A., Robinson, J. P., Royal, M. A., Simon, L., Stauffer, J. W., Stein, W., Tollett, J., \& Witter, J. (2003). Core outcome domains for chronic pain clinical trials: IMMPACT recommendations. Pain, 106(3), 337-345. https://doi.org/10.1016/j.pain.2003.08.001

Turk, D. C., \& Rudy, T. E. (1991). Neglected topics in the treatment of chronic pain patients--relapse, noncompliance, and adherence enhancement. Pain, 44(1), 5-28. https://doi.org/10.1016/0304-3959(91)90142-k

Van der Maas, L. C., Köke, A., Pont, M., Bosscher, R. J., Twisk, J. W., Janssen, T. W., \& Peters, M. L. (2015). Improving the Multidisciplinary Treatment of Chronic Pain by Stimulating Body Awareness: A Cluster-randomized Trial. Clin J Pain, 31(7), 660-669. https://doi.org/10.1097/ajp.0000000000000138

van Hooff, M. L., van der Merwe, J. D., O'Dowd, J., Pavlov, P. W., Spruit, M., de Kleuver, M., \& van Limbeek, J. (2010). Daily functioning and self-management in patients with chronic low back pain after an intensive cognitive behavioral programme for pain management. Eur Spine J, 19(9), 1517-1526. https://doi.org/10.1007/s00586010-1435-5 
van Wilgen, C. P., Dijkstra, P. U., Versteegen, G. J., Fleuren, M. J., Stewart, R., \& van Wijhe, M. (2009). Chronic pain and severe disuse syndrome: long-term outcome of an inpatient multidisciplinary cognitive behavioural programme. J Rehabil Med, 41(3), 122-128. https://doi.org/10.2340/16501977-0292

Vendrig, A. A., Hoofs, M. H., van Akkerveeken, P. F., \& Lamberts-Hopkes, K. J. (2000). [Multidisplinary approach to chronic back pain: postrehabilitation resumption of work the same 3-4 years later as after 6 months]. Ned Tijdschr Geneeskd, 144(46), 2207-2209. (Multidisciplinaire aanpak van chronische rugpijn: werkhervatting na 3-4 jaar hetzelfde als na 6 maanden.)

Verkerk, K., Luijsterburg, P. A., Ronchetti, I., Miedema, H. S., Pool-Goudzwaard, A., van Wingerden, J. P., \& Koes, B. W. (2011). Course and prognosis of recovery for chronic non-specific low back pain: design, therapy program and baseline data of a prospective cohort study. BMC Musculoskeletal Disord, 12, 252. https://doi.org/10.1186/14712474-12-252

Viechtbauer. (2010). Conducting meta-analyses in $\{\mathrm{R}\}$ with the \{metafor $\}$ package. J Statis Software, 36(3), 1-48. https://doi.org/10.18637/jss.v036.i03

Vlaeyen, J. W., Crombez, G., \& Linton, S. J. (2016). The fear-avoidance model of pain. Pain, 157(8), 1588-1589. https:// doi.org/10.1097/j.pain.0000000000000574

Volker, G., van Vree, F., Wolterbeek, R., van Gestel, M., Smeets, R., Köke, A., \& Vlieland, T. V. (2017). Long-Term Outcomes of Multidisciplinary Rehabilitation for Chronic Musculoskeletal Pain. Musculoskeletal Care, 15(1), 59-68. https://doi.org/10.1002/msc.1141

Vowles, K. E., McCracken, L. M., \& O’Brien, J. Z. (2011). Acceptance and values-based action in chronic pain: a threeyear follow-up analysis of treatment effectiveness and process. Behav Res Ther, 49(11), 748-755.

Wagner, F., Janzen, B., Tkachuk, G., Laverty, W., \& Woods, M. (2011). An evaluation of the long-term treatment outcomes of an interdisciplinary chronic pain centre program. J Musculoskeletal Pain, 19(3), 134-143. http:// dx.doi.org/10.3109/10582452.2011.582985

Waterschoot, F. P., Dijkstra, P. U., Hollak, N., de Vries, H. J., Geertzen, J. H., \& Reneman, M. F. (2014). Dose or content? Effectiveness of pain rehabilitation programs for patients with chronic low back pain: a systematic review. Pain, 155(1), 179-189. https://doi.org/10.1016/j.pain.2013.10.006

Westman, A., Linton, S. J., Theorell, T., Ohrvik, J., Wahlén, P., \& Leppert, J. (2006). Quality of life and maintenance of improvements after early multimodal rehabilitation: a 5-year follow-up. Disabil Rehabil, 28(7), 437-446. https://doi.org/10.1080/09638280500192694

Williams, A. C., Eccleston, C., \& Morley, S. (2012). Psychological therapies for the management of chronic pain (excluding headache) in adults. Cochrane Database SystRev, 11(11),Cd007407.https://doi.org/10.1002/14651858. CD007407.pub3

Williamson, P. R., Altman, D. G., Bagley, H., Barnes, K. L., Blazeby, J. M., Brookes, S. T., Clarke, M., Gargon, E., Gorst, S., Harman, N., Kirkham, J. J., McNair, A., Prinsen, C. A. C., Schmitt, J., Terwee, C. B., \& Young, B. (2017). The COMET Handbook: version 1.0. Trials, 18(Suppl 3), 280. https://doi.org/10.1186/s13063-017-1978-4

Wilson, G. T. (2010). Eating disorders, obesity and addiction. Eur Eat Disord Rev, 18(5), 341-351. https://doi. org/10.1002/erv.1048

Wood, W., \& Neal, D. T. (2016). Healthy through habit: Interventions for initiating \& maintaining health behavior change. Behav Sci Pol, 2(1), 71-83.

Zhuk, A., Schiltenwolf, M., \& Neubauer, E. (2018). [Long-term efficacy of multimodal pain therapy for chronic back pain]. Nervenarzt, 89(5), 546-551. https://doi.org/10.1007/s00115-017-0391-2 (Langfristige Wirksamkeit einer multimodalen Schmerztherapie bei chronischen Rückenschmerzen.) 
Appendix 1. Risk of Bias Assessment

\begin{tabular}{|c|c|c|c|c|c|c|c|c|c|c|c|}
\hline Author (year) & 1 & 2 & 3 & 4 & 5 & 6 & 7 & 8 & 9 & 10 & Decision \\
\hline Abbasi (2012) & + & + & + & + & - & - & + & - & + & ? & high \\
\hline Beaudreuil (2010) & + & + & + & - & - & + & + & + & + & ? & high \\
\hline Bendix (1998) & + & + & + & $?$ & + & + & - & + & + & - & high \\
\hline Bendix (2000) & - & - & + & $?$ & + & + & - & + & + & - & high \\
\hline Bergström (2001) & - & + & + & + & + & + & + & + & + & $?$ & high \\
\hline Bergström (2014) & + & + & + & - & + & + & - & + & + & - & high \\
\hline Bileviciute-Ljungar (2014) & - & + & + & + & - & + & + & + & + & - & high \\
\hline Borys (2015) & + & + & + & + & - & - & + & + & + & - & high \\
\hline Brendbekken (2016) & - & - & + & $?$ & - & + & - & + & + & - & high \\
\hline Cardosa (2012) & - & + & + & $?$ & + & + & + & + & + & - & high \\
\hline De Rooij (2014) & + & + & + & + & - & + & + & + & + & + & high \\
\hline Dysvik (2013) & + & + & + & + & + & + & + & + & + & - & high \\
\hline Frost (2000) & + & + & + & - & $?$ & - & - & + & + & - & high \\
\hline Gantschnig (2017) & - & - & + & + & + & + & - & + & + & + & high \\
\hline Gerdle (2016) & - & - & + & + & $?$ & + & + & + & + & - & high \\
\hline Grahn (2000) & + & - & + & + & + & + & + & + & + & - & low \\
\hline Gustafsson (2002) & + & + & + & - & + & + & + & + & + & - & high \\
\hline Hafenbrack (2013) & - & - & + & + & + & + & - & + & + & - & high \\
\hline Haiduk (2017) & + & + & + & + & + & + & $?$ & + & + & $?$ & high \\
\hline Hållstam (2016) & - & + & + & + & $?$ & + & + & + & + & - & high \\
\hline Hazard (1989) & + & + & + & + & - & + & - & + & + & - & high \\
\hline Hildebrandt (1996) & - & + & + & $?$ & $?$ & + & - & + & + & - & high \\
\hline Huffman (2019) & - & - & + & + & + & + & - & + & + & - & high \\
\hline Ibrahim (2019) & - & - & + & + & $?$ & + & - & + & + & - & high \\
\hline Jensen (1997) & - & + & + & - & - & + & + & + & + & + & high \\
\hline Kääpä (2006) & + & + & + & - & + & - & + & + & + & $?$ & high \\
\hline Koopman (2004) & + & + & + & $?$ & $?$ & + & + & + & + & - & high \\
\hline Lemstra (2005) & + & + & + & + & + & + & + & + & + & - & low \\
\hline Letzel (2019) & + & + & + & + & $?$ & + & - & + & + & - & high \\
\hline Mangels (2009) & - & - & + & - & - & + & - & + & + & $?$ & high \\
\hline Martín (2012) & + & + & + & + & + & + & + & - & + & - & high \\
\hline McAllister (2005) & + & + & + & + & $?$ & + & - & + & - & - & high \\
\hline Meng (2011) & - & - & + & $?$ & - & + & - & + & + & - & high \\
\hline Merrick (2009) & + & + & + & + & + & + & + & + & + & - & high \\
\hline Merrick (2012) & + & - & + & + & + & + & + & + & + & + & high \\
\hline Monticone (2013) & + & + & + & + & - & + & + & + & + & + & high \\
\hline
\end{tabular}


Appendix 1. Continued

\begin{tabular}{|c|c|c|c|c|c|c|c|c|c|c|c|}
\hline Author (year) & 1 & 2 & 3 & 4 & 5 & 6 & 7 & 8 & 9 & 10 & Decision \\
\hline Monticone (2016) & + & + & + & + & - & - & + & + & + & - & high \\
\hline Nagel (2009) & - & + & + & + & ? & - & + & + & + & - & high \\
\hline Nicholas (2020) & + & + & + & + & + & + & + & + & + & - & high \\
\hline Olason (2004) & - & + & + & - & - & + & + & + & + & - & high \\
\hline Oslund (2009) & - & + & - & - & ? & + & + & + & + & $?$ & high \\
\hline Persson (2012) & - & + & + & $?$ & $?$ & + & + & + & + & - & high \\
\hline Pietilä-Holmner (2020) & - & $?$ & + & + & + & + & + & + & + & - & high \\
\hline Reck (2017) & - & + & + & + & + & + & + & + & + & - & high \\
\hline Richardson (1994) & - & - & + & $?$ & $?$ & + & - & + & + & - & high \\
\hline Roche-Leboucher (2011) & + & + & - & + & $?$ & - & - & + & + & - & high \\
\hline Semrau (2015) & + & + & + & + & + & + & + & + & + & + & low \\
\hline Silvemark (2014) & - & + & + & + & + & + & + & + & + & - & high \\
\hline Smeets (2008) & + & + & + & + & - & + & + & + & + & + & high \\
\hline Spinhoven (2004) & + & + & + & - & - & + & + & + & + & $?$ & high \\
\hline Stein (2013) & + & + & + & - & - & + & + & - & + & - & high \\
\hline Steinmetz (2019) & + & + & + & $?$ & $?$ & - & - & + & + & - & high \\
\hline Strobel (1998) & - & + & + & $?$ & $?$ & + & + & - & + & - & high \\
\hline Tavafian (2011) & + & + & + & + & + & + & + & + & + & - & low \\
\hline Thieme (2003) & + & + & + & - & - & + & + & + & + & - & high \\
\hline Van der Maas (2015) & - & + & + & + & - & + & + & + & + & + & high \\
\hline van Hooff (2010) & + & + & + & + & - & + & + & + & + & + & high \\
\hline van Wilgen (2009) & + & + & + & - & $?$ & + & + & + & + & - & high \\
\hline Vendrig (2000) & - & + & + & + & + & + & + & + & + & - & high \\
\hline Verkerk (2011) & + & + & + & - & - & + & + & + & + & $?$ & high \\
\hline Volker (2017) & + & + & + & + & + & + & + & + & + & + & low \\
\hline Vowles (2011) & + & + & + & + & + & + & + & + & + & + & low \\
\hline Wagner (2011) & - & - & + & + & - & + & - & + & + & $?$ & high \\
\hline Westman (2006) & - & - & + & + & $?$ & + & - & - & + & - & high \\
\hline Zhuk (2018) & + & + & + & $?$ & $?$ & + & - & + & + & - & high \\
\hline
\end{tabular}




\section{SUPPLEMENTARY MATERIALS}

This chapter contains the following supplementary materials

- The multimedia appendix

- $\quad$ Supplement 1: Search string

- Supplement 2: Data extraction form

- $\quad$ Supplement 3: R code (version 202001101)

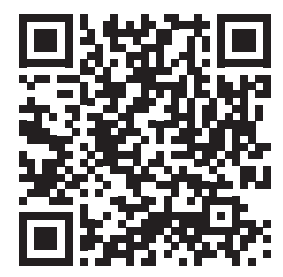

The multimedia appendix can be accessed via this QR code or link https:/datascience.hu.nl/rsconnect/impt-cohorts/

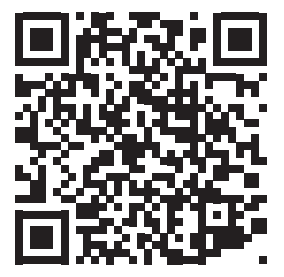

All other supplementary materials can be accessed via this QR code or link https://github.com/stefanelbers/doctoral_thesis/ 


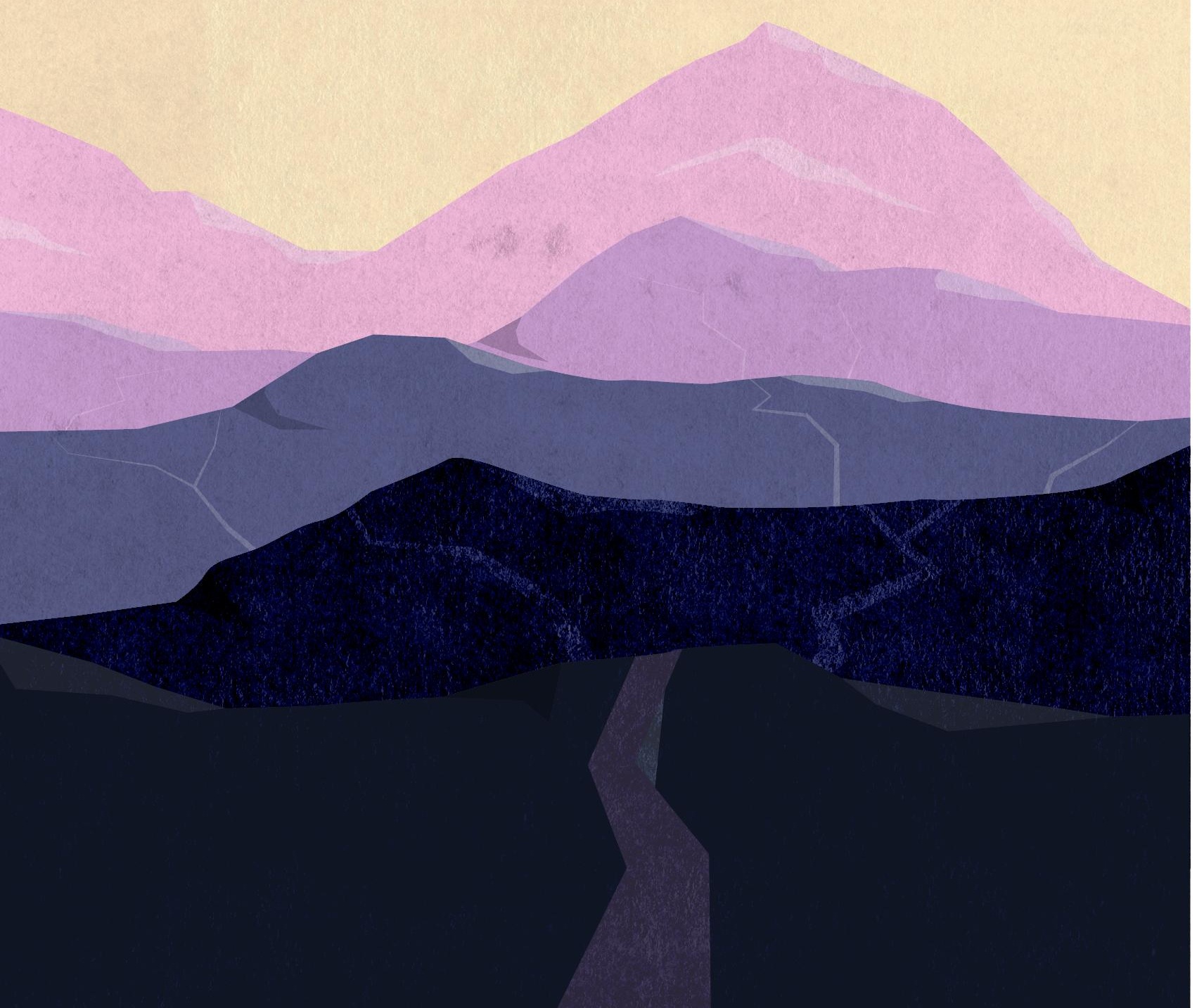




\title{
03
}

\section{Living Systematic Reviews in Rehabilitation Science can Improve Evidence-Based Healthcare}

\author{
S. Elbers ${ }^{1,2}$, H. Wittink ${ }^{1}$, U. Kaiser ${ }^{3},{ }^{4}$, J. Kleijnen ${ }^{5}$, J. Pool ${ }^{1}$, A. Köke ${ }^{2,6,7}$, R. Smeets ${ }^{2,8}$
}

\section{Submitted}

1. Research group Lifestyle \& Health, Research Centre Healthy and Sustainable Living, University of Applied Sciences Utrecht, The Netherlands.

2. Department of Rehabilitation Medicine, Research School CAPHRI, Faculty of Health, Life Sciences and Medicine, Maastricht University, The Netherlands.

3. Comprehensive Pain Center, Medical Faculty Technical University Dresden, Germany.

4. University Hospital Carl Gustav Carus Dresden, Germany.

5. Department of Family Medicine, Research School CAPHRI, Faculty of Health, Life Sciences and Medicine, Maastricht University, The Netherlands.

6. Centre of Expertise in Pain and Rehabilitation, Adelante, Hoensbroek, The Netherlands.

7. South University of Applied Sciences Heerlen, The Netherlands.

8. CIR Revalidatie, location Eindhoven, The Netherlands. 


\section{ABSTRACT}

Although systematic reviews are considered as central components in evidence-based practice, they currently face an important challenge to keep up with the exponential publication rate of clinical trials. After initial publication, only a minority of the systematic reviews are updated and it often takes multiple years before these results become accessible. Consequently, many evidence syntheses in systematic reviews are not up to date, thereby increasing the time-gap between research findings and clinical practice. A potential solution is offered by a living systematic reviews approach. These types of studies are characterized by a workflow of continuous updates which decreases the time it takes to disseminate new findings. Although living systematic reviews are specifically designed to continuously synthesize new evidence in rapidly emerging topics, they have also considerable potential in slower developing domains, such as rehabilitation science. In this commentary we outline the rationale and required steps to transition a regular systematic review into a living systematic review. We also propose a workflow that is designed for rehabilitation science.

Keywords: Systematic review; Living systematic review; Rehabilitation; Chronic pain; Methods; Meta-analysis. 


\section{BACKGROUND}

Systematic reviews are considered to be the foundations of evidence-based practice (Chandler \& Hopewell, 2013; Shojania et al., 2007; Stevens, 2001). The structured method of identifying, appraising, evaluating and synthesizing primary research findings facilitates clinical decision making based on the available evidence at a given time (Boutron et al., 2020; Simmonds et al., 2017). Despite its widespread success, researchers have identified several problems in the current conduct of systematic reviews. First, the average duration of performing a systematic review is almost a year from initial search to publication (Chandler \& Hopewell, 2013). Furthermore, only a minority of the published reviews are updated and the median estimated time to update is approximately 3 years for Cochrane reviews and 5 years for non-Cochrane reviews (Bashir et al., 2018; Rombey et al., 2020). Given the continuously increasing flow of new trials, many systematic reviews become rapidly out of date (Bastian et al., 2010; Boutron et al., 2020). Second, poorly prepared systematic reviews are susceptible to bias, such as incomplete search strategies (Salvador-Oliván et al., 2019), errors in data extraction (Horton et al., 2010), and inter-rater disagreement in risk of bias assessments (Hartling et al., 2009). Although the effect on outcomes may be limited (Jones et al., 2005), the current system does not easily allow for corrections or updated practices after publication of the initial results.

Living systematic reviews (LSRs) are proposed as an alternative method to traditional systematic reviews that may offer a solution to these challenges. A systematic review is considered "living" when it includes a system by which it continuously incorporates newly available evidence into the analysis (Elliott et al., 2017). Generally, this system consists of a predefined cyclic workflow with planned updates at intervals ranging from days to months. Assisted by the availability of recent software developments that provide (semi) assisted solutions for the review process, these updates take only a fraction of the time of conducting a base review (Créquit et al., 2020; Thomas et al., 2017). The chosen timeframe is at the discretion of the researchers and generally depends on the speed at which a topic of interest is expected to develop. Although LSRs have specifically been designed for rapid updating cycles of emerging topics, such as mapping the COVID-19 trials (COVID-NMA), protocols have been published that include updating cycles of 12 months (De Zeeuw et al., 2020; Pollock \& van Wijck, 2019; Winters et al., 2018).

The aim of this commentary is to illustrate the process of transitioning a regular review into a LSR and to propose a workflow for updating LSRs over time. We will use a recently performed research project where we transitioned a regular systematic review into a LSR as an example to discuss the required steps and key issues. 


\section{MAIN TEXT}

\section{Characteristics of the base review}

We performed the initial review in September 2020 (the protocol was registered in PROSPERO under CRD42018076093) to identify the change in outcomes over time and the betweenstudy heterogeneity for interdisciplinary pain treatment programs for patients with chronic pain. All longitudinal study designs such as case series and RCTs were considered. Our main reason for including more study types than RCTs was that carefully controlled inclusion criteria and treatment procedures do often not reflect the heterogeneous reality of clinical practice in the domain of rehabilitation (Morley et al., 2013; Rowbotham et al., 2013; Smeets, 2021; Wade et al., 2010). In addition, many treatment programs disseminate their results through case series, which contain important information regarding the change over time. The abstract screening, full-text selection, data extraction, and risk of bias assessment was performed in duplicate. Primary studies were eligible if they included IMPT programs as intervention for patients with chronic primary pain that was primarily perceived in musculoskeletal structures. Furthermore, studies had to include a baseline and a follow-up assessment of at minimum 12 months post treatment for at least one of the outcomes of interest: Physical function, pain interference, self-efficacy, depression, anxiety, anger, general emotional function, social role functioning and pain intensity. We assessed Risk of Bias with the JBI critical appraisal checklist for case series. Our data analysis included the change over time per outcome, by calculating standardized mean differences between pre, post and final follow-up timepoints.

\section{LSR protocol}

A LSR starts with a baseline systematic review that is in line with PRISMA guidelines (Counotte et al., 2018; Elliott et al., 2017). In addition, there should be a study protocol that includes a rationale for maintaining a LSR as well as planned methodological and statistical approaches that are in line with multiple iterations over time (Elliott et al., 2017). For example, repeating meta-analyses at each update may inflate type I error and should be taken into account (Simmonds et al., 2017).

When the decision was made for the transition of this systematic review into a living systematic review, we submitted a new study protocol in PROSPERO (CRD42021247142). We chose to create a new record instead of updating the record of the base review, because the workflow and iterative nature of the living review are substantially different. The main rationale for this living systematic review was to provide and maintain an up-todate overview of all interdisciplinary pain treatment programs that include one of our outcomes of interest for at least 12 months. Other reasons included a faster inclusion of 
primary studies in a meta-analysis, the possibility of accommodating new methodological developments into the study, and to update data extraction forms at a later stage, to include follow-up studies or correct omissions or errors.

\section{Workflow}

LSRs require long-term commitment of a research team, which can lead to a substantial ongoing workload (Millard et al., 2019). Detailed planning of a feasible workflow that allows for updates of the procedures over time is therefore recommended (e.g. Counotte et al., 2018; Schmidt et al., 2020). Researchers should anticipate that the research team is likely to change over time, and include training programs and calibration procedures. This is also true for leaving the possibility open to integrate new digital developments to (semi) assisted approaches within the review process at a later stage (Elliott et al., 2017; Thomas et al., 2017).

Figure 1 depicts the workflow diagram for the current project. Each year, we will rerun the search in all databases. New records will be imported into Endnote and deduplicated using the procedure of Bramer and colleagues (2016). The set of unique records will then be imported into web application Rayyan, for masked screening in duplicate (Ouzzani et al., 2016). Potential eligible studies will be imported into Endnote to help find the full text versions for these references. We will use Google Forms to perform the second-round selection, data extraction and risk of bias assessment. The advantage of these digital forms is that they are stored in an online spreadsheet that is connected to our digital application. Hence, new studies are automatically integrated into the online tables and calculations. The results of the update will be evaluated by the steering committee during the yearly meeting.

The involved researchers are divided over two teams. Members of the steering committee will meet on a yearly base and decide whether new results justify a peer review publication, based on the number of new cohorts, changes in outcomes or conclusions. At minimum, we will publish an update every three years because we believe that an absence of any change also contains valuable information. The steering committee will also discuss potential methodology improvements or amendments to the procedures, including the opportunity to integrate large scale routinely collected data (Créquit et al., 2020), the refinement of the data extraction form base on a newly developed checklist (Negrini et al., 2020), and initiatives to increase participation of the research community (e.g. Probst et al., 2019). Finally, the steering committee will evaluate the need for continuation of this LSR project based on the replication checklist by Tugwell and colleagues (2020). The meeting notes will be added as appendix to the online repository. Members of the review team will be responsible for screening, study selection, data extraction and risk of bias assessment. They 
will receive training in all software applications and review procedures. A test dataset that is derived from the base review of 20 studies that includes 3 included studies will be used to calibrate the procedures. As recommended by Gagnier and colleagues (2013), we aim to always include members with expertise in pain rehabilitation.

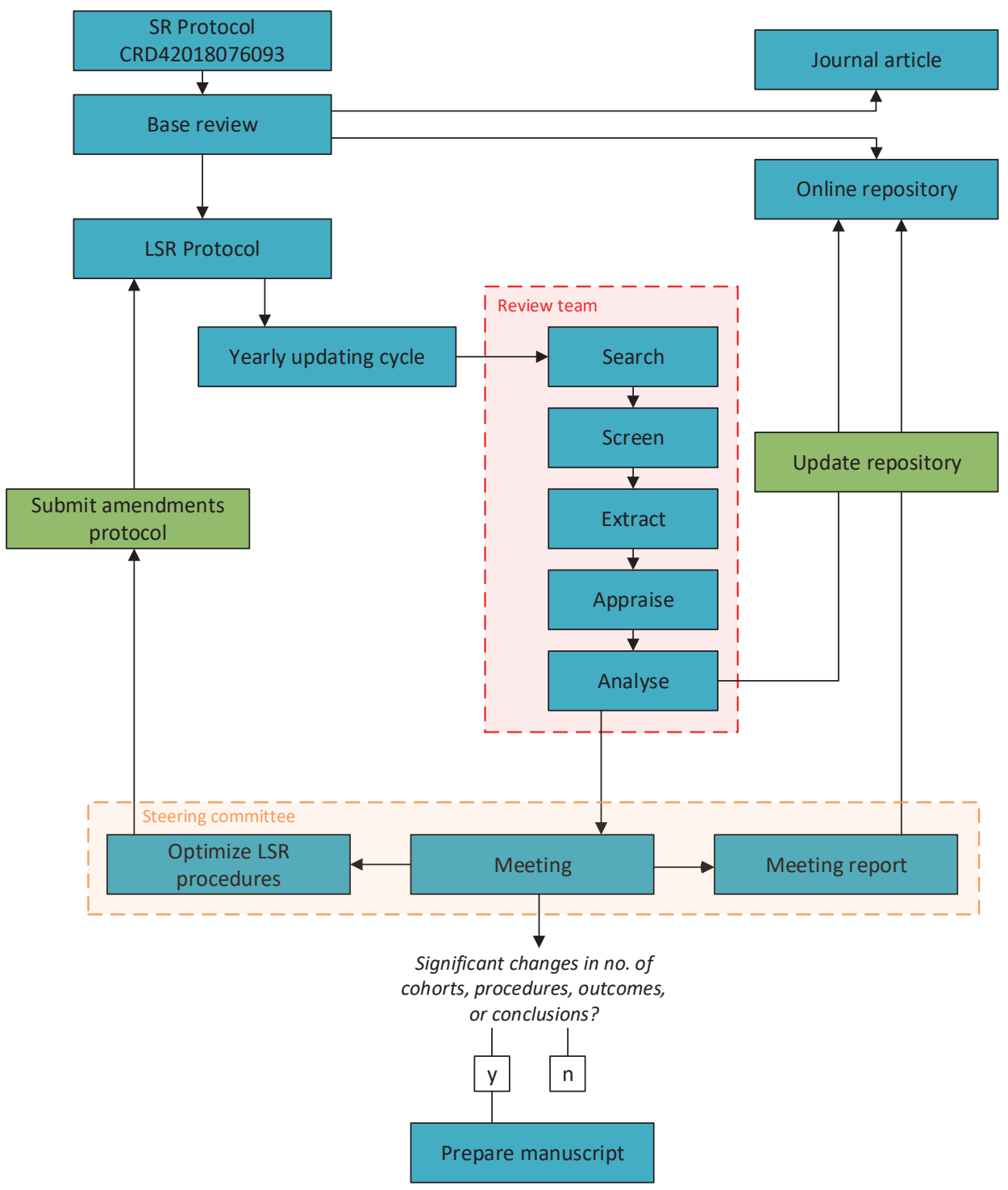

Figure 1. Workflow Diagram of the Living Systematic Review. 


\section{Dissemination}

In addition to journal articles, LSR results are often disseminated through online applications (e.g. Matl et al., 2017; Probst et al., 2019). These digital platforms include all extracted data as well as interactive functions to visualize or summarize (a subset of) the data that is specifically relevant to the end-user (Boutron et al., 2020; Matl et al., 2017; Probst et al., 2019). Consequently, these interactive functions provide the opportunity to supply information to various stakeholders, including researchers, clinicians, policy makers and patients.

To disseminate the results an in interactive way, we developed an R Shiny application that provides relevant and most up to date information of this review project (Elbers, 2020). The online platform contains key tables, time series and forest plots. To optimize transparency, the raw data extraction forms and risk of bias assessments are also made available. Using functionalities of Shiny software, all plots and figures include interactive functions, including tables with search, filter and sort functions, forest plots with adjustable corrections for within-subject correlations and time series, with cohort selection and export functions. These functions help users to navigate to the available data, to explore differences and similarities between cohorts and to perform sensitivity analyses. The source code is available through GitHub, which also registers the amendments that will be made over time (Elbers, 2021).

\section{CONCLUSIONS}

Innovations in (semi-)assisted tools for conducting a systematic review have streamlined the overall process and have substantially reduced the efforts to update results over time. LSRs provide a method to adopt these new methods to speed up progress and to improve procedures over time, while simultaneously uphold the scientific rigor of systematic reviews. Consequently, these up-to-date overviews, communicated through peer reviewed articles and online applications, provide the opportunity to increase the dissemination and implementation of scientific findings into clinical practice. 


\section{REFERENCES}

Bashir, R., Surian, D., \& Dunn, A. G. (2018). Time-to-update of systematic reviews relative to the availability of new evidence. Syst Rev, 7(1), 195. https://doi.org/10.1186/s13643-018-0856-9

Bastian, H., Glasziou, P., \& Chalmers, I. (2010). Seventy-five trials and eleven systematic reviews a day: how will we ever keep up? PLoS med, 7(9), e1000326. https://doi.org/10.1371/journal.pmed.1000326

Boutron, I., Créquit, P., Williams, H., Meerpohl, J., Craig, J. C., \& Ravaud, P. (2020). Future of evidence ecosystem series: 1. Introduction-Evidence synthesis ecosystem needs dramatic change. J Clin Epidem. https://doi. org/10.1016/j.jclinepi.2020.01.024

Bramer, W. M., Giustini, D., de Jonge, G. B., Holland, L., \& Bekhuis, T. (2016). De-duplication of database search results for systematic reviews in EndNote. J Med Lib Ass, 104(3), 240. https://doi.org/10.5195/jmla.2016.24

Chandler, J., \& Hopewell, S. (2013). Cochrane methods-twenty years experience in developing systematic review methods. Syst Rev, 2, 76. https://doi.org/10.1186/2046-4053-2-76

Counotte, M. J., Egli-Gany, D., Riesen, M., Abraha, M., Porgo, T. V., Wang, J., \& Low, N. (2018). Zika virus infection as a cause of congenital brain abnormalities and Guillain-Barré syndrome: From systematic review to living systematic review. F1000Res, 7. https://doi.org/10.12688/f1000research.13704.1

Créquit, P., Boutron, I., Meerpohl, J., Williams, H., Craig, J., \& Ravaud, P. (2020). Future of evidence ecosystem series: 2. Current opportunities and need for better tools and methods. J clin epidim. https://doi.org/10.1016/j. jclinepi.2020.01.023

De Zeeuw, T. I., Peters, G., Baan, A., de Regt, L., Bolman, C., \& Crutzen, R. (2020). Extending the Earcheck intervention: behavior change to prevent hearing damage, with a focus on young people with low social-economic status. Retrieved 7-1-2020 from https://osf.io/7xjhg/?branch=master

Elbers. (2020). Interactive dashboard: IMPT cohorts. Retrieved 10-03-2021 from https://datascience.hu.nl/rsconnect/ impt-cohorts/

Elbers, S. (2021). impt meta_analysis, Github repository. https://github.com/stefanelbers/impt.meta_analysis

Elliott, J. H., Synnot, A., Turner, T., Simmonds, M., Akl, E. A., McDonald, S., Salanti, G., Meerpohl, J., MacLehose, H., \& Hilton, J. (2017). Living systematic review: 1. Introduction-the why, what, when, and how. J Clin Epidem, 91, 23-30. https://doi.org/ 10.1016/j.jclinepi.2017.08.010

Gagnier, J. J., Morgenstern, H., Altman, D. G., Berlin, J., Chang, S., McCulloch, P., Sun, X., \& Moher, D. (2013). Consensus-based recommendations for investigating clinical heterogeneity in systematic reviews. BMC med red methodol, 13(1), 106. hhttps://doi.org/10.1186/1471-2288-13-106

Hartling, L., Ospina, M., Liang, Y., Dryden, D. M., Hooton, N., Seida, J. K., \& Klassen, T. P. (2009). Risk of bias versus quality assessment of randomised controlled trials: cross sectional study. Bmj, 339, b4012. https://doi-org. ezproxy.ub.unimaas.nl/10.1136/bmj.b4012

Horton, J., Vandermeer, B., Hartling, L., Tjosvold, L., Klassen, T. P., \& Buscemi, N. (2010). Systematic review data extraction: cross-sectional study showed that experience did not increase accuracy. J Clin Epidem, 63(3), 289298. https://10.1016/j.jclinepi.2009.04.007

Jones, A. P., Remmington, T., Williamson, P. R., Ashby, D., \& Smyth, R. L. (2005). High prevalence but low impact of data extraction and reporting errors were found in Cochrane systematic reviews. J Clin Epidem, 58(7), 741-742. https://10.1016/j.jclinepi.2004.11.024

Matl, S., Brosig, R., Baust, M., Navab, N., \& Demirci, S. (2017). Vascular image registration techniques: A living review. Med Image Anal, 35, 1-17. https://doi.org/10.1016/j.media.2016.05.005 
Millard, T., Synnot, A., Elliott, J., Green, S., McDonald, S., \& Turner, T. (2019). Feasibility and acceptability of living systematic reviews: results from a mixed-methods evaluation. Syst Rev, 8(1), 325. https://doi.org/10.1186/ s13643-019-1248-5

Morley, S., Williams, A., \& Eccleston, C. (2013). Examining the evidence about psychological treatments for chronic pain: Time for a paradigm shift? Pain, 154(10), 1929-1931. https://doi.org/10.1016/j.pain.2013.05.049

Negrini, S., Armijo-Olivo, S., Patrini, M., Frontera, W. R., Heinemann, A. W., Machalicek, W., Whyte, J., \& Arienti, C. (2020). The randomized controlled trials rehabilitation checklist: Methodology of development of a reporting guideline specific to rehabilitation. Am J Phys Med Rehabil, 99(3), 210-215. https://doi.org/10.1097/ PHM.0000000000001370

Ouzzani, M., Hammady, H., Fedorowicz, Z., \& Elmagarmid, A. (2016). Rayyan-a web and mobile app for systematic reviews. Syst Rev, 5(1), 210. https://doi.org/10.1186/s13643-016-0384-4

Pollock, A., \& van Wijck, F. (2019). Cochrane overviews: how can we optimize their impact on evidence-based rehabilitation? Eur J Phys Rehabil Med, 55, 395-410. https://10.23736/S1973-9087.19.05780-0

Probst, P., Hüttner, F. J., Meydan, Ö., Kalkum, E., Kretschmer, R., Jensen, K., Kenngott, H. G., Mihaljevic, A. L., Thilo, H., \& Büchler, M. W. (2019). Evidence map of pancreatic surgery: protocol for a living systematic review and meta-analysis. BMJ open, 9(9), e032353. https://doi.org/10.1136/bmjopen-2019-032353

Rombey, T., Lochner, V., Puljak, L., Könsgen, N., Mathes, T., \& Pieper, D. (2020). Epidemiology and reporting characteristics of non-Cochrane updates of systematic reviews: A cross-sectional study. Res Synt Meth, 11(3), 471-483. https://doi-org.ezproxy.ub.unimaas.nl/10.1002/jrsm.1409

Rowbotham, M. C., Gilron, I., Glazer, C., Rice, A. S., Smith, B. H., Stewart, W. F., \& Wasan, A. D. (2013). Can pragmatic trials help us better understand chronic pain and improve treatment? Pain, 154(5), 643-646. https://doi. org/10.1016/j.pain.2013.02.034

Salvador-Oliván, J. A., Marco-Cuenca, G., \& Arquero-Avilés, R. (2019). Errors in search strategies used in systematic reviews and their effects on information retrieval. J Med Lib Ass, 107(2), 210. https://10.5195/jmla.2019.567

Schmidt, L., Olorisade, B. K., McGuinness, L. A., Thomas, J., \& Higgins, J. P. (2020). Data extraction methods for systematic review (semi) automation: A living review protocol. F1000Res, 9. https://10.12688/ f1000research.22781.2

Shojania, K. G., Sampson, M., Ansari, M. T., Ji, J., Doucette, S., \& Moher, D. (2007). How quickly do systematic reviews go out of date? A survival analysis. Ann Int Med, 147(4), 224-233. https://doi.org/10.7326/0003-4819-147-4200708210-00179

Simmonds, M., Salanti, G., McKenzie, J., Elliott, J., Agoritsas, T., Hilton, J., Perron, C., Akl, E., Hodder, R., \& Pestridge, C. (2017). Living systematic reviews: 3. Statistical methods for updating meta-analyses. Journal Clin Epidem, 91, 38-46. https://10.1016/j.jclinepi.2017.08.008

Smeets, R. J. (2021). How to sustain and improve client centred (matched) care in chronic musculoskeletal pain? Start by changing the way policy makers select and judge the large amount of available data, and get rid of the dogma of stepped care. Eur J Phys, 23(2), 66-67. https://doi.org/10.1080/21679169.2021.1888469

Stevens, K. R. (2001). Systematic reviews: the heart of evidence-based practice. AACN Adv Crit Care, 12(4), 529-538. https://doi.org/10.1097/00044067-200111000-00009

Thomas, J., Noel-Storr, A., Marshall, I., Wallace, B., McDonald, S., Mavergames, C., Glasziou, P., Shemilt, I., Synnot, A., \& Turner, T. (2017). Living systematic reviews: 2. Combining human and machine effort. J Clin Epidem, 91, 31-37. https://doi.org/ 10.1016/j.jclinepi.2017.08.011

Tugwell, P., Welch, V. A., Karunananthan, S., Maxwell, L. J., Akl, E. A., Avey, M. T., Bhutta, Z. A., Brouwers, M. C., Clark, J. P., \& Cook, S. (2020). When to replicate systematic reviews of interventions: consensus checklist. Bmj, 370. https://doi.org/ 10.1136/bmj.m2864 
Wade, D. T., Smeets, R. J., \& Verbunt, J. A. (2010). Research in rehabilitation medicine: methodological challenges. J Clin Epidem, 63(7), 699-704. https://10.1016/j.jclinepi.2009.07.010

Winters, M., Holden, S., Vicenzino, B., Welton, N. J., Caldwell, D. M., Lura, C. B., Weir, A., \& Rathleff, M. S. (2018). Which treatment is most effective for patients with patellofemoral pain? A protocol for a living systematic review including network meta-analysis. BMJ open, 8(11). https://doi.org/10.1136/bmjopen-2018-022920 



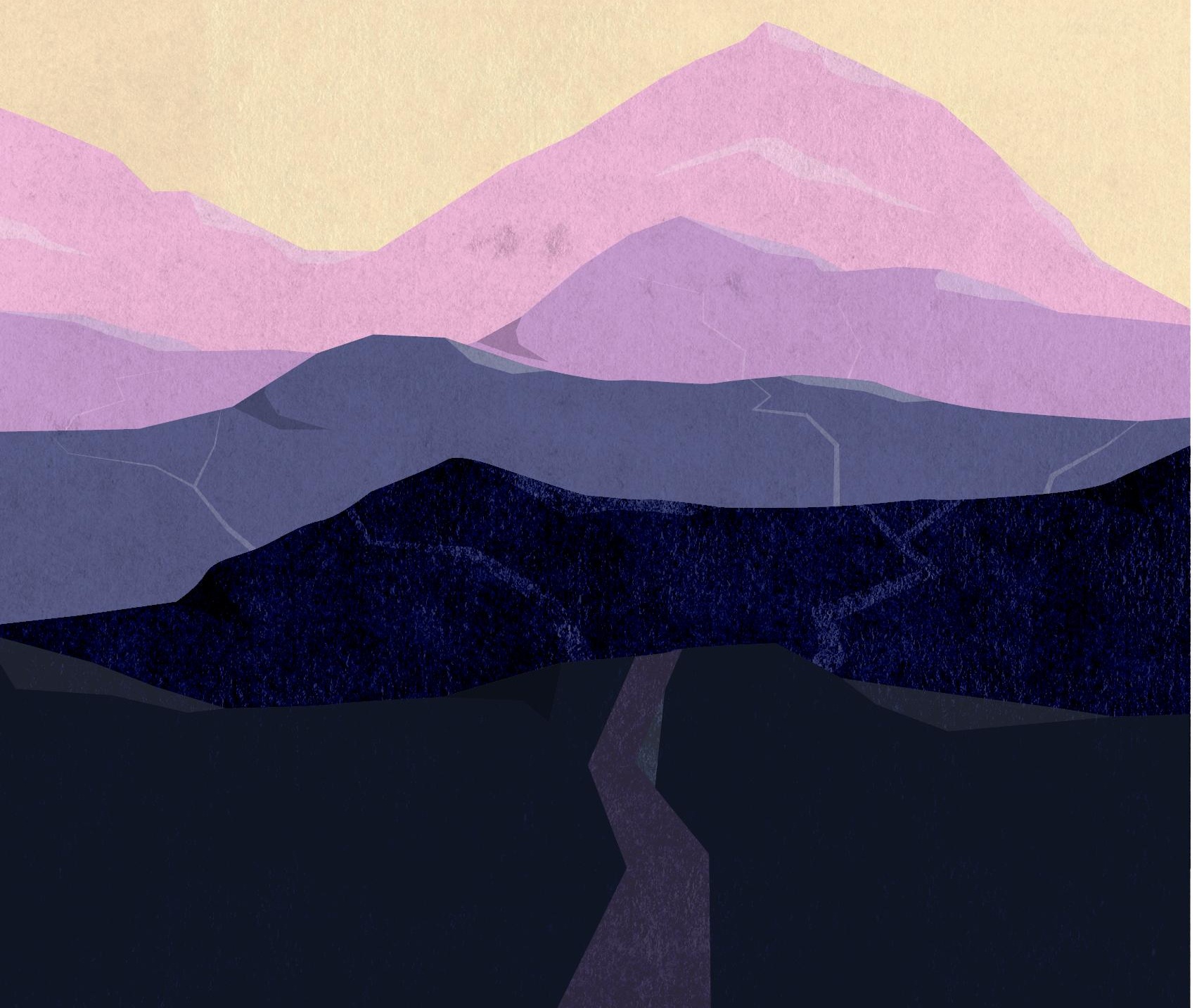




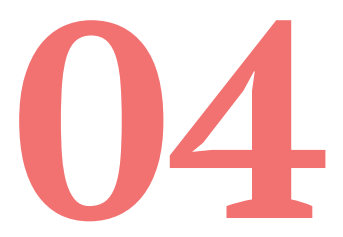

\section{The Effectiveness of Generic Self- Management Interventions for Patients with Chronic Musculoskeletal Pain on Physical Function, Self-Efficacy, Pain Intensity and Physical Activity:}

\section{A Systematic Review and Meta-Analysis}

S. Elbers ${ }^{1,2}$, H. Wittink ${ }^{1}$, J. Pool ${ }^{1}$, R. Smeets ${ }^{2,3}$

European Journal of Pain (2018), 22(9), 1577-1596

1. Research group Lifestyle \& Health, Research Centre Healthy and Sustainable Living, University of Applied Sciences Utrecht, The Netherlands.

2. Department of Rehabilitation Medicine, Research School CAPHRI, Faculty of Health, Life, Sciences and Medicine, Maastricht University, The Netherlands.

3. CIR Revalidatie, location Eindhoven, The Netherlands. 


\section{ABSTRACT}

Generic self-management programs aim to facilitate behavioural adjustment and therefore have considerable potential for patients with chronic musculoskeletal pain. Our main objective was to collect and synthesize all data on the effectiveness of generic selfmanagement interventions for patients with chronic musculoskeletal pain in terms of physical function, self-efficacy, pain intensity and physical activity. Our secondary objective was to describe the content of these interventions, by means of classification according to the Behaviour Change Technique Taxonomy. We searched PubMed, CENTRAL, Embase and Psycinfo for eligible studies. Study selection, data extraction and risk of bias were assessed by two researchers independently. Meta-analyses were only performed if the studies were sufficiently homogeneous and GRADE was used to determine the quality of evidence. We identified 20 randomized controlled trials that compared a self-management intervention to any type of control group.

For post-intervention results, there was moderate quality evidence of a statistically significant but clinically unimportant effect for physical function and pain intensity, both favouring the self-management group. At follow-up, there was moderate quality evidence of a small clinically insignificant effect for self-efficacy, favouring the self-management group. All other comparisons did not indicate an effect. Classification of the behaviour change techniques showed large heterogeneity across studies. These results indicate that generic self-management interventions have a marginal benefit for patients with chronic musculoskeletal pain in the short-term for physical function and pain intensity and for self-efficacy in the long-term, and vary considerably with respect to intervention content.

Significance: This study contributes to a growing body of evidence that generic self-management interventions have limited effectiveness for patients with chronic musculoskeletal pain. Furthermore, this study has identified substantial differences in both content and delivery mode across self-management interventions. 


\section{INTRODUCTION}

Chronic musculoskeletal pain negatively influences daily life functioning, emotional well-being and social participation (Turk et al., 2011). Low back pain and neck pain alone contribute to 1694 years lost to disability (YLD) per 100,000 persons annually, placing these conditions, respectively, first and fourth in the ranking of diseases on global years lived with disability (Vos et al., 2012).

The experience of pain interrupts individuals' ongoing activities, forcing them to choose between pursuit of their intended action or activity, disengagement or avoidance behaviours. Motivational conflicts such as these constantly interfere with daily life activities and are assumed to have a negative effect on an individual's well-being and identity (Vlaeyen et al., 2016). In order to maintain sufficient quality of life, successful self-management - the ability to manage symptoms, treatment, physical, psychological and social consequences, and lifestyle changes related to one's chronic condition - is essential (Barlow et al., 2002; Lorig and Holman, 2003). To facilitate this process, generic self-management interventions are designed to teach persons how to self-regulate their chronic condition. Rather than providing unilateral solutions to disease-specific problems, self-management interventions provide a generic set of skills and competencies (e.g. problem solving, decision making, etc.) in order to facilitate living a meaningful life despite chronic pain.

The definition of self-management does not specify how this behavioural adjustment should be achieved. This allows for a large variety of content and delivery modes in selfmanagement interventions. In order to provide more clarity in comparing interventions, Michie and colleagues have developed a taxonomy of behaviour change techniques (BCTs) that enables more precise reporting (Michie et al., 2013). Moreover, classification of components according to this taxonomy facilitates comparison of intervention content and is expected to provide insight into the various intended mechanisms of action.

As self-management programmes aim to facilitate behavioural adjustment, they have considerable potential for positive long-term effects on outcomes of importance to patients. However, as newly learned behaviours in the context of physical activity (Sullum et al., 2000) or pain rehabilitation (Turk and Rudy, 1991) are difficult to maintain, it is important to study the long-term outcomes of these interventions.

\section{The Present Study}

Our primary aim was to collect and synthesize all available data on the immediate and long-term (more than six months) effectiveness of generic self-management interventions for patients with chronic musculoskeletal pain in terms of physical function, self-efficacy, 
pain intensity and physical activity. We hypothesized that self-management interventions would improve self-efficacy, enabling patients with chronic pain to increase their physical activity, consequently reducing their perceived limitations in physical function, at least in the short-term. Attributable to a shift in attention from disease-related problems to engagement in daily life activities, patients might even perceive less pain after the intervention.

Our secondary aim was to describe the intervention content, by means of classification according to the Behaviour Change Technique Taxonomy (v1). This aim builds on recent efforts to acquire more insight into theory and techniques behind self-management interventions (e.g. Keogh et al., 2015).

\section{METHOD}

\section{Protocol and Registration}

The review protocol has been registered in the Prospero database (CRD42015024417).

\section{Information Sources}

We searched MEDLINE via PubMed, CENTRAL, Embase and Psycinfo databases for eligible studies from inception up to May 2017. The search strategy was designed in collaboration with a medical informatics specialist and contained a combination of thesaurus terms and free text words. The PubMed search string (see Supplement 1) was constructed first and was used as a template for the other databases. The database search was extended in the following ways: first, reference lists of included articles were screened by one of the researchers (SE) and eligible studies underwent the same reviewing process (i.e. backward citation tracking). Second, when a study was included in the analysis, PubMed was used to search for eligible studies that cited this study (i.e. forward citation tracking). Third, to minimize publication bias, we also searched for unpublished studies and grey literature in DART-Europe E-thesis portal, the Open Access Thesis and Dissertations database (OATD), the WHO International Clinical Trials Registry Platform (WHO-ICTRP), and the Networked Digital Library of Theses and Dissertations (NDLTD) with the combined terms 'chronic pain' and 'self-management' as entry terms.

\section{Eligibility Criteria}

We included randomized controlled trials that met the following eligibility criteria: The study sample had to consist of adult patients with chronic musculoskeletal pain, defined as pain that persists for longer than 3 months and that is perceived in the musculoskeletal 
system (i.e. bones, joints, tendons or muscles). Although self-management principles have been incorporated in multicomponent treatment programmes (e.g. Meng et al., 2011; Du et al., 2017), and self-management skill training can overlap with other types of interventions with different underlying theoretical approaches (e.g. action planning in the Health Action Process Approach, (Schwarzer, 2017), we were only interested in generic interventions that focused on improving behavioural adjustment by training self-management skills. Therefore, the intervention had to address at least one of the following five self-management skills: problem-solving, decision making, resource utilization, forming a partnership with a health care provider and taking action (Lorig and Holman, 2003). In addition, the intervention had to include both an element of information transfer on self-management principles (e.g. education session or lecture) and a training component where self-management skills were actually rehearsed or performed. The intervention had to be focused on improving generic self-management skills, rather than on training disease-specific skills (e.g. joint protection techniques). The study had to include a control intervention that was not a selfmanagement intervention. Lastly, the study had to include at least one of the following outcome measures: physical function, self-efficacy, pain intensity, or physical activity. For physical function, we included self-report instruments that measured the degree of interference that chronic pain had on daily life activities and social participation. For self-efficacy, we included self-report instruments that measured the level of confidence in patients' capabilities to perform daily life tasks or activities. Pain intensity measures were included if they solely measured the degree of pain experienced on a scale from low to high intensity. Composite scores of various moments of pain intensity were also included (e.g. Von Korff scales), as well as sum scores of pain intensity for each tender point. For physical activity, we included both self-report instruments and activity trackers that provided an indication of how often certain types of physical activities were performed.

We excluded studies with samples that solely consisted of patients with osteoarthritis, because Kroon et al. (2014) had recently published a systematic review of self-management interventions for this subgroup. When a composite sample included patients with osteoarthritis, at least $50 \%$ of the sample had to consist of patients with other forms of chronic musculoskeletal pain. In addition, interventions that were designed to improve self-management in the context of pre-operative training, post-operative rehabilitation or palliative care were excluded, as we expected that this would lead to substantial heterogeneity regarding disease management and coping. To avoid heterogeneity, studies were also excluded if they only included patients on the basis of a specific comorbidity (e.g. psychiatric or obese patients), or if they combined the self-management intervention with other chronic pain treatment modalities (e.g. graded activity, exposure in vivo, Acceptance and Commitment Therapy, interdisciplinary pain management programmes). We also excluded e-health interventions that did not include any form of face-to-face contact 
during treatment, because a recent systematic review had been conducted on this topic (Eccleston et al., 2014). Only studies that were published in Dutch or English languages were included. We used the online application software 'Rayyan' to screen the abstracts (Ouzzani et al., 2016).

\section{Study Selection, Data Collection and Risk of Bias}

Two researchers independently (HW and SE) performed the study selection, data collection and assessment of risk of bias in five stages. For each stage (abstract screening; full text inclusion; BCT data extraction; patient, intervention, comparison, outcome and study design data extraction; risk of bias assessment), we held pilot test sessions where we calibrated our procedures. At regular intervals within each stage, meetings were held to compare results and to reach consensus. If differences in scoring remained, a third researcher (JP) made the final decision. In the first stage, all abstracts were screened on eligibility criteria with respect to study design and patients. In the second stage, full text articles were read and checked on all eligibility criteria. Data collection started in the third stage and involved (1) copying all information regarding the intervention that was provided in the study or in the protocol; (2) extracting all individual intervention components from this information; and (3) classifying these components according to the BCT taxonomy v1 (Michie et al., 2013). In the fourth stage, we extracted all relevant data with respect to our analysis, including patient characteristics, means and standard deviations for all outcome measures of interest. For each study, we selected the measures that best fitted our definition for the primary outcomes. In accordance with the Cochrane Handbook, we considered studies as our primary source of interest (Higgins and Green, 2011). As a consequence, we also extracted data from study protocols and articles with follow-up data, when they were available. In the fifth stage, we determined risk of bias using the Cochrane's Collaboration's tool for assessing risk of bias. The following types of bias were assessed: random sequence generation (selection bias); allocation concealment (selection bias); blinding of outcome assessment (detection bias); incomplete outcome data (attrition bias), selective reporting (reporting bias) and other sources of bias. Blinding of participants and personnel was not included in the bias assessment, as the characteristics of self-management interventions do not allow for appropriate blinding. Due to the nature of the studies, we scored the default blinding of outcome assessment as high risk of bias, but upgraded to unclear or low if attempts to blind the outcome assessment for patients or assessors were described (e.g. blinding of patients to former assessment). All other types of bias were assessed according to the guidelines in the Cochrane Handbook (Higgins and Green, 2011). The risk of bias was used as input for the assessment of the quality of evidence for each outcome measure. Studies were considered high risk of bias when three or more items were scored unclear or high, or when two items were scored high. 


\section{Outcome Reporting and Data Synthesis}

Between-group comparisons for post-intervention (within one month of the end of the intervention) and follow-up (at minimum six months post-intervention) were calculated per study for each of the outcomes of interest, using RevMan 5.3 software (Cochrane, 2011). In case of more than one follow-up measurement, we included the last time point in our analysis. If more than one self-management group was included within a study, we used only the intervention group that best fitted our definition of self-management interventions. In the situation of more than one control group within a study, we included only the most active control group in our comparisons. Results were presented for each outcome separately. If the GRADE analyses revealed both directness and consistency as a serious risk of bias, we concluded that the data were too heterogeneous to perform a metaanalysis and presented the results narratively. Each outcome was expected to be measured with differing varying questionnaires. Therefore, standardized mean differences (SMD) with $95 \%$ confidence intervals were used. A priori, we decided to select random effects models because we assumed differences in the true outcomes across studies, based on between-study variation in duration, intensity and patient characteristics. If the pooled SMD was significant, we re-expressed this effect on one of the outcome measures to examine the clinical importance. This was performed by multiplying the SMD with the standard deviation of the control group of one of the included studies that adopted this measure. Subsequently, we compared this effect with available estimates of the minimal important change to assess the clinical importance. When it was not possible to obtain measures of central tendency or dispersion, the results were narratively presented and compared to the results of the meta-analysis.

BCTs were graphically visualized in a table. Relative differences between studies and between domains of the taxonomy were calculated and presented narratively.

\section{Assessment of the Quality of Evidence}

For each comparison in the meta-analysis, we used the GRADEpro Guideline Development Tool (Evidence Prime I, 2015) to determine the quality of evidence. As only randomized controlled trials were included, the initial quality of evidence started as 'high' and was downgraded as a result of limitations with respect to risk of bias, inconsistency, indirectness, imprecision or publication bias.

For each comparison, we downgraded the level of evidence when (1) more than 25\% of the sample came from studies with high risk of bias; (2) the $I^{2}$ was more than $60 \%$ combined with a limited overlap of confidence intervals (inconsistency); (3) substantial differences were present in study population, intervention protocol, control group or 
outcome measures (indirectness); or (4) when the total sample size of all included studies was less than the optimal information size of $n=400$ (imprecision). We determined the optimal information size with a sample size calculation with $\alpha=0.05, \beta=0.8, \mathrm{SD}=0.2$ as parameters (Schünneman et al., 2013). To assess publication bias, funnel plot symmetry and distribution of effect sizes were inspected. We based our quality of evidence criteria on the Grade Handbook (Schünneman et al., 2013) and the Cochrane Handbook (Higgins and Green, 2011).

\section{RESULTS}

\section{Study Selection}

The search yielded 7843 hits. After removal of duplicates and the screening of abstracts, 102 full-text articles were assessed for eligibility. Eighty-two studies were excluded and 20 studies were selected for data extraction and analysis (see Figure 1).

\section{Patient and Study Characteristics}

The total study population consisted of 3557 patients. Seventy-five percent of the study population was female. All studies were performed in Western Europe, Australia or the United States. Average pain duration characteristics were only reported in eight studies and the means ranged from 2.3 to 20 years with a median of 8.4 years. Patient eligibility criteria varied across studies and were based on localization (e.g. back pain), specific diagnosis group (e.g. fibromyalgia syndrome), or duration of pain. Table 1 provides an overview of all participant characteristics within each study.

The included studies show substantial variation regarding intervention content, delivery and measurement instruments (table 2). For example, the median number of face-toface sessions was 6 (range $=3-15$ ), and the median duration was $15 \mathrm{~h}$ (range: $2.8-45 \mathrm{~h}$ ). Furthermore, fifteen studies included a follow-up measurement of at least six months postintervention, with a mean of $10.53(S D=2.59)$ months. The mean number of BCTs was 12.6 (range $=5-26$ ). Forty-three of the 93 available BCTs in the taxonomy were identified in the studies and we identified BCTs in all domains of the taxonomy, except for scheduled consequences and covert learning. The domains with the highest numbers of BCTs were goals and planning (accounting for $27.8 \%$ of the total BCTs), and social support (10.6\%). Six BCTs were frequently used in the interventions: 'Social support (unspecified) provided by group interventions', 'credible source provided by an experienced health care provider or patient', and 'goal setting (behaviour)' were present in at least $90 \%$ of the interventions. 
'Problem-solving', 'instruction on how to perform the behaviour' and 'information about health consequences (education)' were present in 80-90\% of the interventions. Supplement 2 provides a full overview of the BCT profiles per study.

\section{Studies identified through database screening:}

Central: 1643

Embase: 2316

Psyclnfo: 2466

PubMed: 2466
Forward citation tracking: 142

Backward citation tracking: 22 Grey Literature: 1

Total: 7669

Total: 165

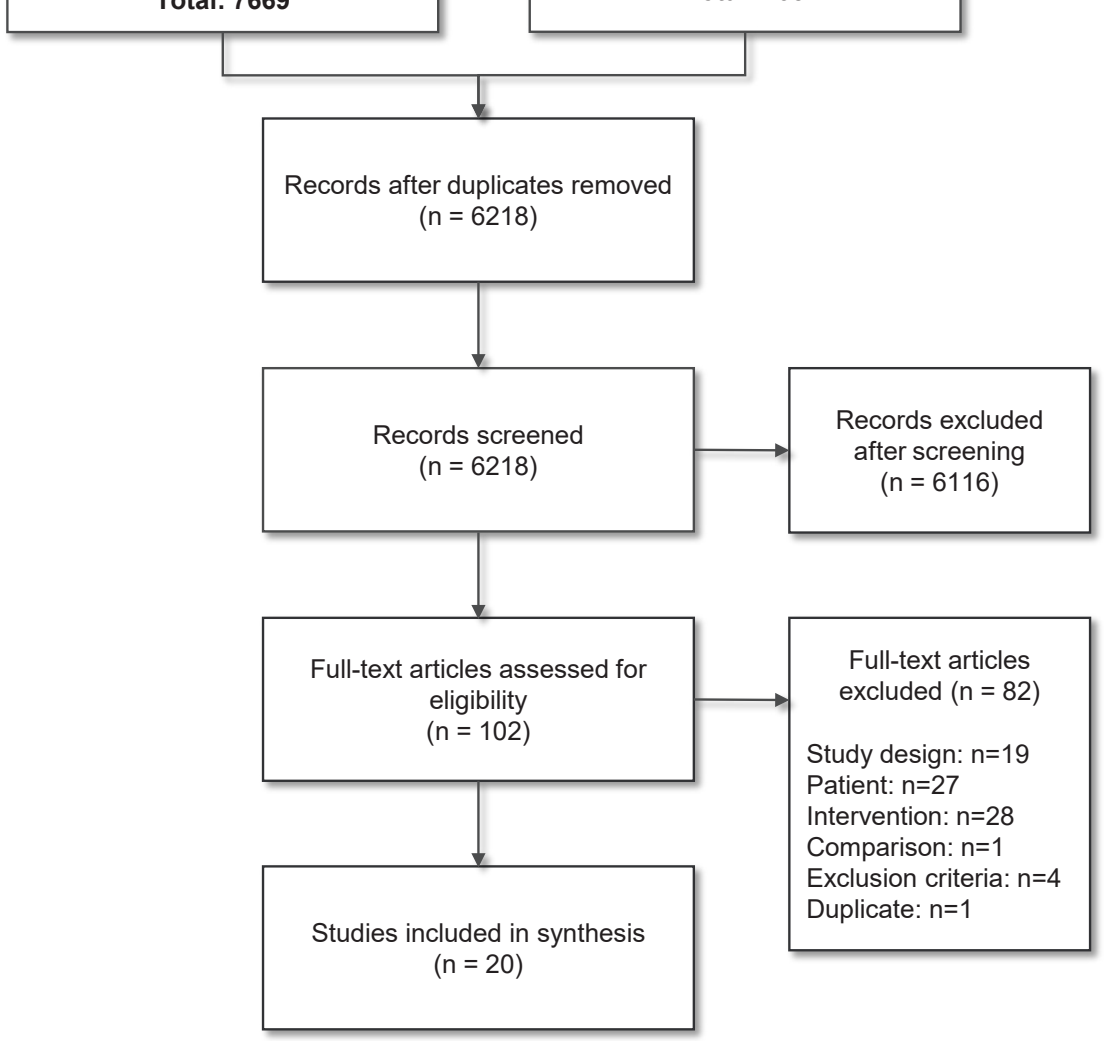

Figure 1. Flowchart of the Literature Search and Study Selection.

Notes: $\mathrm{n}$ is the number of randomized controlled trials. FCT, forward citation tracking; BCT, backward citation tracking; Grey Lit, grey literature. 
Table 1. Characteristics of Participants Within the Included Studies

\begin{tabular}{|c|c|c|c|c|c|c|}
\hline \multirow[b]{2}{*}{ Study } & \multicolumn{3}{|c|}{ Age } & \multicolumn{3}{|c|}{ Pain duration } \\
\hline & $(N)$ & $M(S D)$ & $\%$ Female & Country & $M(S D)$ & Diagnosis group \\
\hline $\begin{array}{l}\text { Andersen } \\
(2015)\end{array}$ & 141 & $\begin{array}{c}45.23 \\
(10.49)\end{array}$ & 55.71 & Denmark & NR & $\begin{array}{c}\text { Back pain or upper body } \\
\text { pain }\end{array}$ \\
\hline $\begin{array}{l}\text { Arvidson } \\
\text { (2013) }\end{array}$ & 202 & $\begin{array}{c}55.48 \\
(12.05)\end{array}$ & 73 & Sweden & NR & Rheumatic diseases \\
\hline $\begin{array}{l}\text { Asenlof } \\
(2005)\end{array}$ & 122 & 42,56 & 77.3 & Sweden & $M d n=2.3^{\mathrm{a}}$ & Musculoskeletal pain \\
\hline $\begin{array}{l}\text { Burckhardt } \\
\text { (1994) }\end{array}$ & 99 & $\begin{array}{l}46.5 \\
(8.3)\end{array}$ & 100 & Sweden & $7.5(5.5)$ & Fibromyalgia syndrome \\
\hline $\begin{array}{l}\text { Dworkin } \\
\text { (2002) }\end{array}$ & 124 & $\begin{array}{c}37.7 \\
(30.61)\end{array}$ & 84.68 & United States & NR & $\begin{array}{c}\text { Temporo-mandibular } \\
\text { disorders }\end{array}$ \\
\hline $\begin{array}{l}\text { Ersek } \\
(2008)\end{array}$ & 256 & $\begin{array}{l}81.85 \\
(6.48)\end{array}$ & 84.75 & United States & NR & Chronic pain (> 3 months) \\
\hline $\begin{array}{l}\text { Grønning } \\
(2012)\end{array}$ & 141 & $\begin{array}{c}58 \\
(11)\end{array}$ & 69 & Norway & $12(13)$ & Polyarthritis \\
\hline $\begin{array}{l}\text { Haas } \\
(2005)\end{array}$ & 120 & $\begin{array}{l}77.2 \\
(7.7)\end{array}$ & 84.4 & United States & NR & Low back pain \\
\hline $\begin{array}{l}\text { Hutting } \\
\text { (2015) }\end{array}$ & 123 & $\begin{array}{c}46.24 \\
(10.89)\end{array}$ & 75.9 & Netherlands & NR & $\begin{array}{c}\text { chronic non-specific } \\
\text { CANS }\end{array}$ \\
\hline $\begin{array}{l}\text { King } \\
(2002)\end{array}$ & 196 & $\begin{array}{l}46.07 \\
(9.05)\end{array}$ & 100 & Canada & $9.33(9.22)$ & Fibromyalgia syndrome \\
\hline $\begin{array}{l}\text { Knittle } \\
(2015)\end{array}$ & 78 & $\begin{array}{c}62.75 \\
(11.79)\end{array}$ & 66.69 & Netherlands & NR & Rheumatoid arthritis \\
\hline $\begin{array}{l}\text { Lefort } \\
\text { (1998) }\end{array}$ & 110 & $\begin{array}{c}39.48 \\
(25-60)^{\mathrm{b}}\end{array}$ & 75 & Canada & $\begin{array}{c}6.07 \\
(1-28)^{\mathrm{b}}\end{array}$ & $\begin{array}{l}\text { Non-malignant chronic } \\
\text { pain (> 3-6 months) }\end{array}$ \\
\hline $\begin{array}{l}\text { Linton } \\
\text { (1997) }\end{array}$ & 103 & $\begin{array}{l}50.73 \\
(9.71)\end{array}$ & 73.68 & Sweden & NR & Musculoskeletal pain \\
\hline $\begin{array}{l}\text { Manning } \\
\text { (2014) }\end{array}$ & 108 & $\begin{array}{c}55.07 \\
(15.55)\end{array}$ & 75.93 & United Kingdom & $20(18.52)$ & Rheumatoid arthritis \\
\hline $\begin{array}{l}\text { Moore } \\
(2000)\end{array}$ & 226 & $\begin{array}{c}49.5 \\
(10.6)\end{array}$ & 54 & United States & NR & Back pain \\
\hline $\begin{array}{l}\text { Nicholas } \\
(2013)\end{array}$ & 141 & $\begin{array}{l}73.9 \\
(6.5)\end{array}$ & 63 & Australia & $14.83(17.3)$ & Chronic pain (> 6 months) \\
\hline $\begin{array}{l}\text { Stuifbergen } \\
\text { (2010) }\end{array}$ & 234 & $\begin{array}{l}53.09 \\
(9.86)\end{array}$ & 100 & United States & NR & Fibromyalgia syndrome \\
\hline $\begin{array}{l}\text { Taal } \\
(1993)\end{array}$ & 75 & $\begin{array}{c}44.94 \\
(24-64)^{\mathrm{b}}\end{array}$ & 73.68 & Netherlands & $\begin{array}{c}4.3 \\
(1-29)^{\mathrm{b}}\end{array}$ & Rheumatoid Arthritis \\
\hline $\begin{array}{l}\text { Taylor } \\
(2016)\end{array}$ & 703 & $\begin{array}{c}59.78 \\
(13.67)\end{array}$ & 67 & United Kingdom & NR & $\begin{array}{c}\text { Chronic Musculoskeletal } \\
\text { Pain }\end{array}$ \\
\hline $\begin{array}{l}\text { Von Korff } \\
\text { (1998) }\end{array}$ & 255 & $\begin{array}{c}49.8 \\
(11.3)\end{array}$ & 62.37 & United States & NR & Back Pain \\
\hline
\end{tabular}

Notes: $a=$ median; $b=$ range. $\mathrm{NR}=$ not reported. 


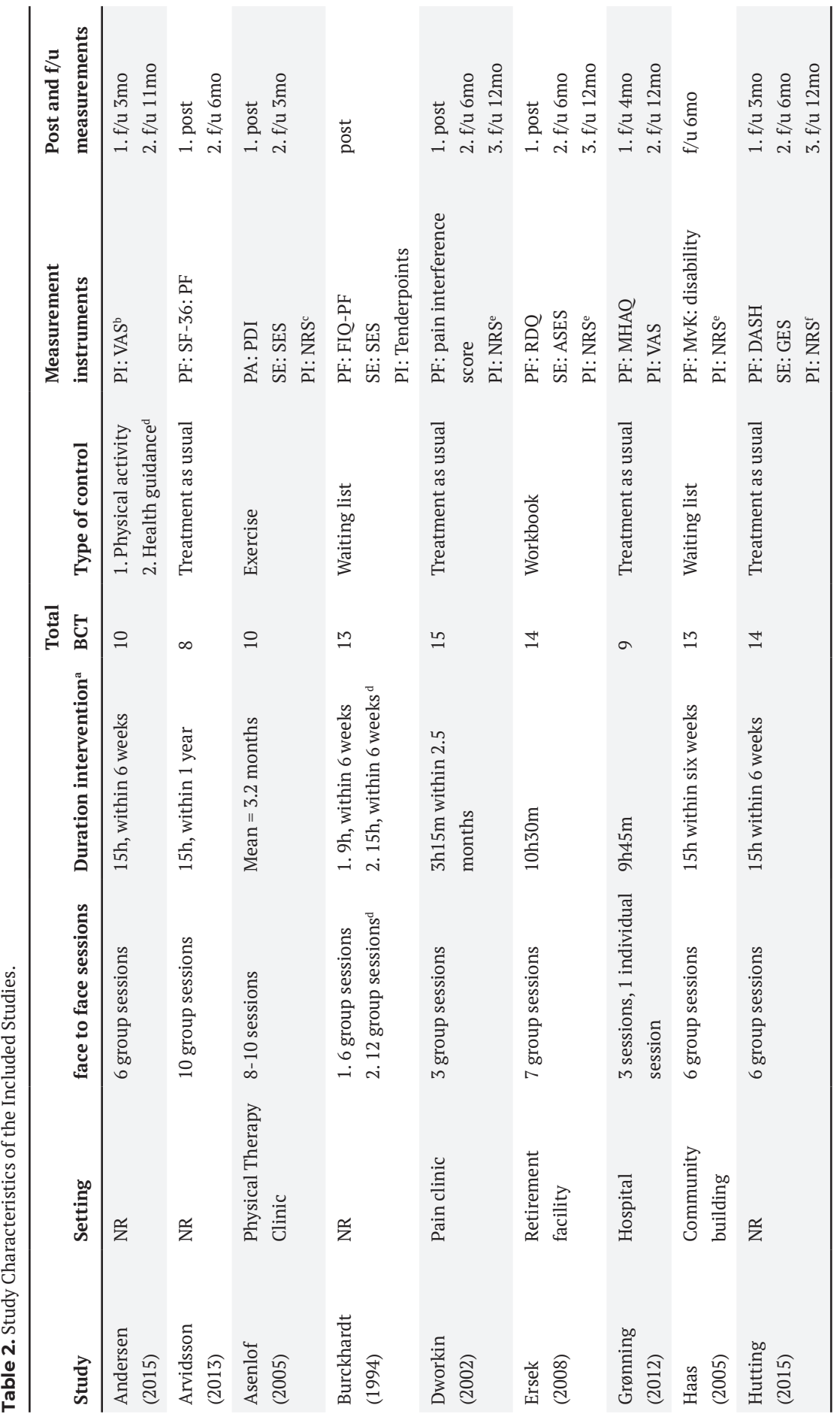




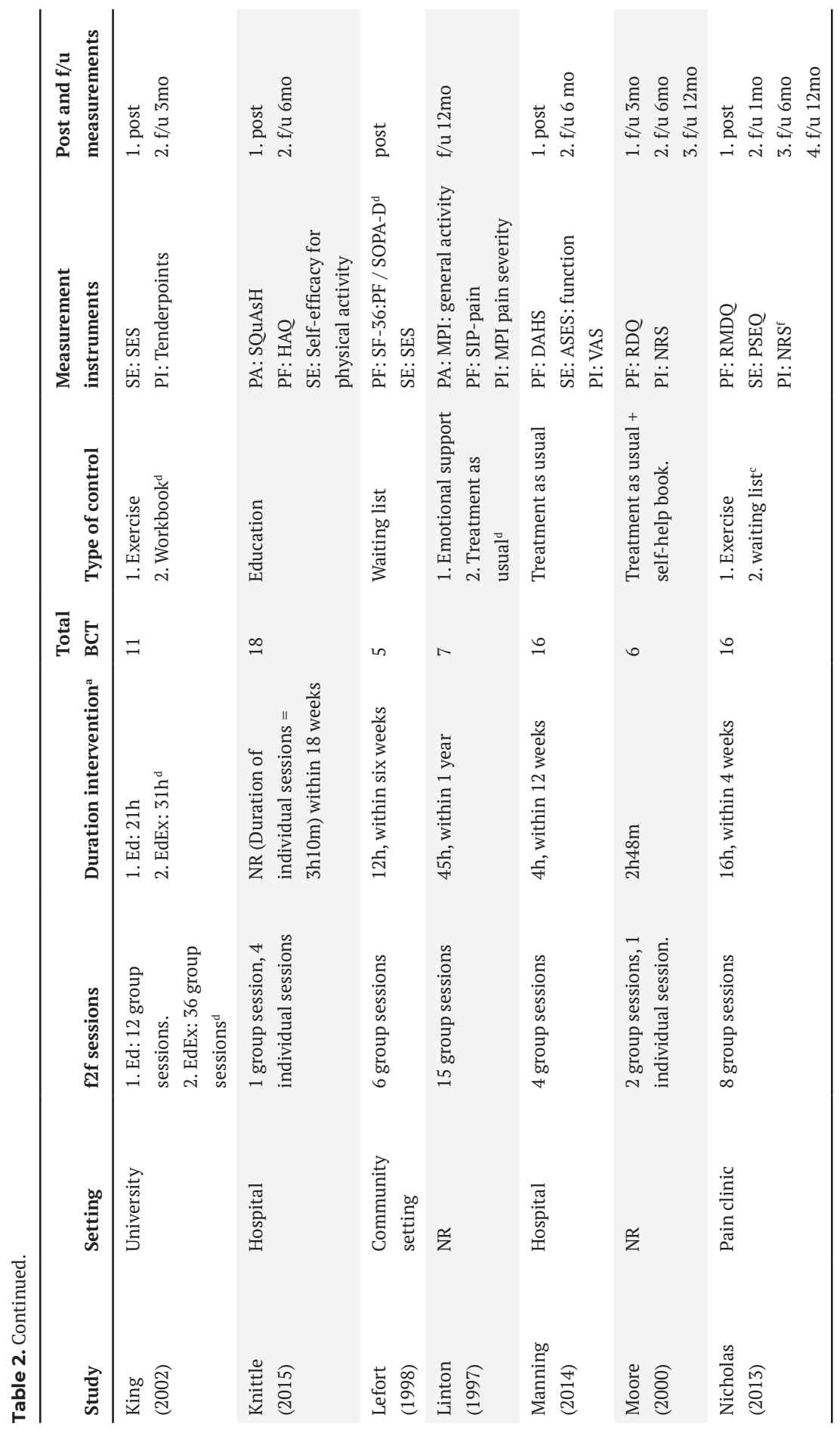




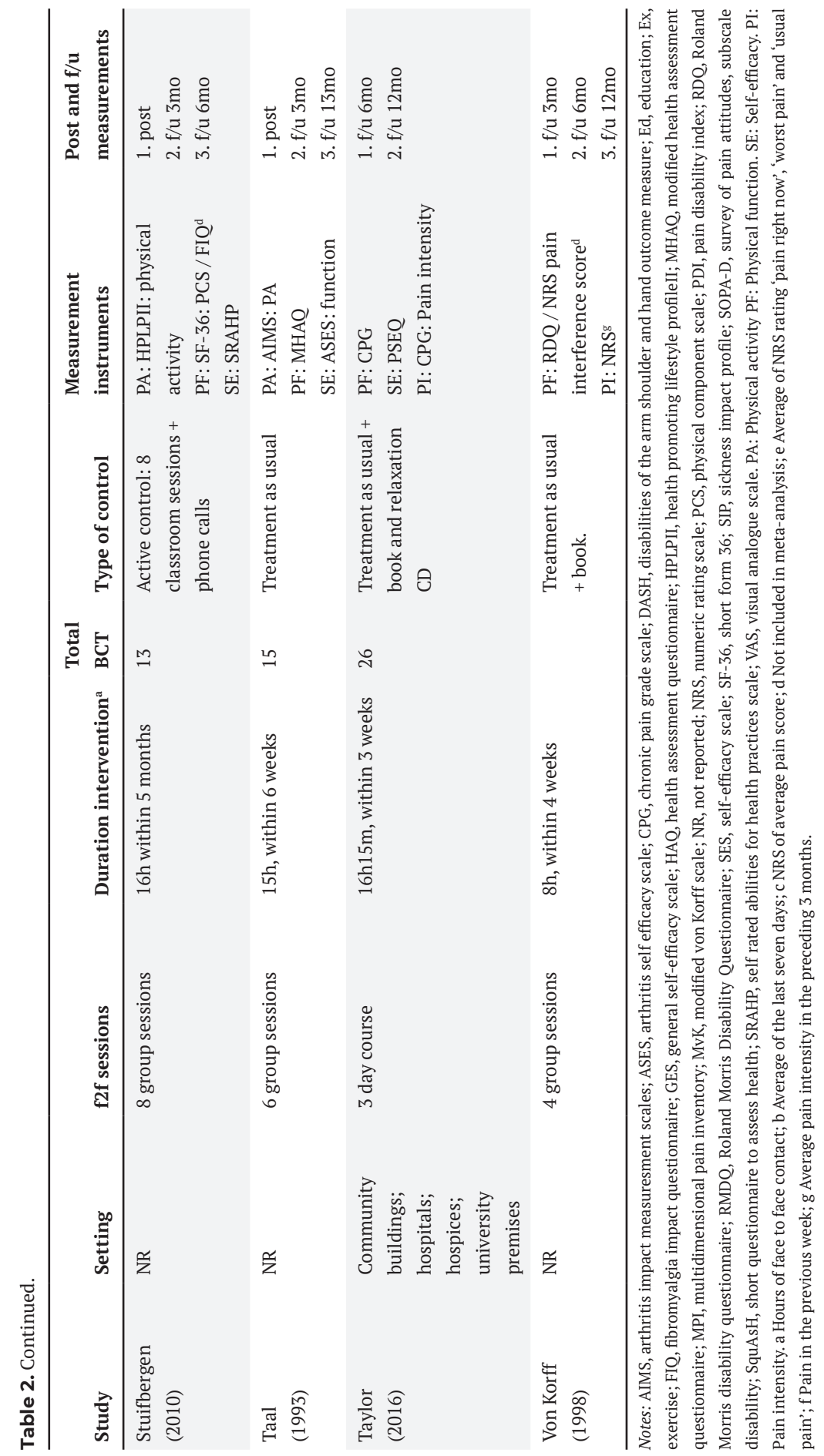


Sixteen studies provided sufficient data to perform meta-analyses. One of these studies used change scores to control for baseline differences (Manning et al., 2014), whereas the other studies used final value scores of their outcome measures. As both type of scores are not compatible within one calculation of a standardized mean difference, we analysed the comparisons of Manning et al. (2014) separately. The four studies that could not be included in the meta-analyses were presented narratively (Taal et al., 1993; Burckhardt et al., 1994; Dworkin et al., 2002; Hutting et al., 2015).

\section{Risk of Bias}

The risk of bias was low for all studies, except for Asenlof et al. (2005), Taal et al. (1993), Dworkin et al. (2002), Burckhardt et al. (1994), and Von Korff et al. (1998). Table 3 shows all risk of bias assessments.

Table 3. Risk of Bias Assessment of Included Studies.

\begin{tabular}{|c|c|c|c|c|c|c|}
\hline Study & 1 & 2 & 3 & 4 & 5 & 6 \\
\hline Andersen (2015) & + & + & + & + & + & + \\
\hline Arvidsson (2013) & + & + & $?$ & - & + & + \\
\hline Asenlof (2005) & + & $?$ & - & - & + & + \\
\hline Burckhardt (1994) & $?$ & $?$ & $?$ & - & $?$ & + \\
\hline Dworkin (2002) & $?$ & $?$ & ? & - & - & + \\
\hline Ersek (2008) & + & + & - & ? & + & + \\
\hline Grønning (2012) & + & + & - & + & + & ? \\
\hline Haas (2005) & + & + & $?$ & + & $?$ & + \\
\hline Hutting (2015) & + & + & + & + & ? & ? \\
\hline King (2002) & + & + & + & $?$ & + & + \\
\hline Knittle (2015) & + & + & - & + & + & ? \\
\hline Lefort (1998) & + & + & + & + & + & + \\
\hline Linton (1997) & + & + & $?$ & + & + & + \\
\hline Manning (2014) & + & + & + & + & + & + \\
\hline Moore (2000) & $?$ & $?$ & + & + & + & + \\
\hline Nicholas (2013) & + & + & + & $?$ & + & + \\
\hline Stuifbergen (2010) & + & + & + & + & + & + \\
\hline Taal (1993) & $?$ & $?$ & $?$ & - & + & - \\
\hline Taylor (2016) & + & + & - & + & + & + \\
\hline Von Korff (1998) & $?$ & ? & + & + & + & ? \\
\hline
\end{tabular}

1: Random sequence allocation; (selection bias) 2: allocation concealment (selection bias); 3: blinding of outcome assessment (detection bias); 4: Incomplete outcome data (attrition bias); 5: Selective reporting (reporting bias); 6: Other bias. 


\section{Immediate Post-Intervention Comparisons}

Physical Function. For eight of the 11 studies that reported outcomes on physical function we were able to calculate standardized mean differences. Statistical pooling was considered appropriate despite a high $I^{2}$, as a sensitivity analysis revealed that the heterogeneity was mainly attributable to one study (Asenlof et al., 2005), and that the confidence intervals showed substantial overlap. The pooled effect was calculated with Hedges' (adjusted) $g$ and was significant; $\mathrm{SMD}=-0.28$ [ $-0.52,-0.03], \mathrm{z}=2.23, \mathrm{p}=0.03$ (see Figure 2 ). When this effect is re-expressed on a Pain Disability Index (PDI), using the baseline standard deviation ( $S D$ = 14.7) of the control group of Asenlof et al. (2005), this effect corresponds to a betweengroup difference of 4.12 points on a PDI favouring the self-management group. This is lower than the minimal clinically important change of 8.5 points that was calculated by Soer et al. (2012). The between-group comparison of post minus pre scores of Manning et al. (2014) was not significant; SMD $=-0.40[-0.82,0.02], \mathrm{z}=1.88, \mathrm{p}=0.06$. Burckhardt et al. (1994) and Dworkin et al. (2002) reported no between-group differences post-intervention. Taal et al. (1993) found a statistically significant difference on the Modified Health Assessment Questionnaire (M-HAQ), with the experimental group showing a mean decrease of 0.01 points and the control group showing a mean increase of 0.16 points compared to baseline scores $(\mathrm{p}<0.05)$.

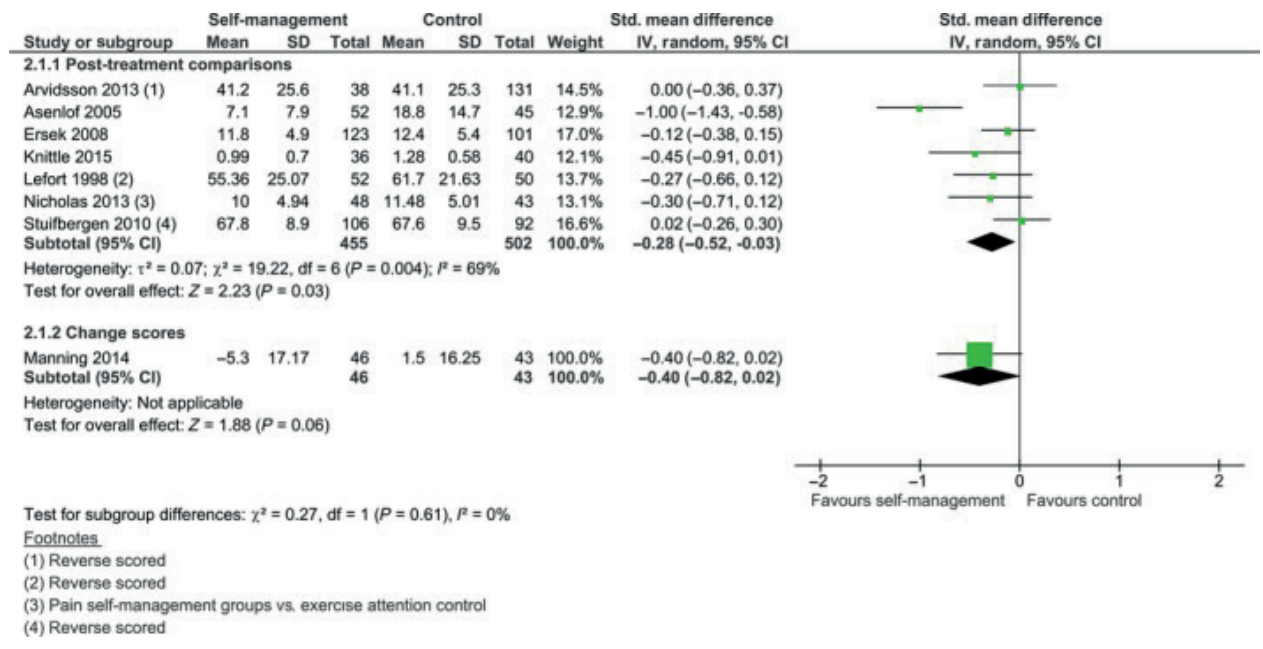

Figure 2. Post Treatment Comparison of Self-Management Intervention Versus Control on Physical Function. 
Self-Efficacy. Ten studies reported post-intervention comparisons for self-efficacy. However, due to the statistical heterogeneity $\left(I^{2}=65 \%, \chi 2(7965)=17.39, \mathrm{p}=0.02\right)$, an overall effect was not calculated. Four comparisons showed statistically significant differences, favouring the experimental group (Lefort et al., 1998; Asenlof et al., 2005; Ersek et al., 2008; Nicholas et al., 2013) with SMD ranging from -0.74 to -0.32 . Furthermore, Taal et al. (1993) reported an effect favouring the self-management intervention, with a change score of the experimental group (0.17) that significantly differed from the change score of the control group (-0.13), $\mathrm{p}<0.05$. Burckhardt et al. (1994) also found a statistically significant difference between both groups, with the self-management group reporting higher scores on the function subscale of the self-efficacy scale (620.7), than the control group (467.5). Four comparisons did not show an effect, (King et al., 2002; Stuifbergen et al., 2010; Manning et al., 2014; Knittle et al., 2015). The unpooled comparisons, indicating a trend favouring self-management, are shown in Figure 3.

\begin{tabular}{|c|c|c|c|c|c|c|c|c|c|c|}
\hline \multirow[b]{2}{*}{ Study or subgroup } & \multicolumn{3}{|c|}{ Self-management } & \multicolumn{2}{|c|}{ Control } & \multicolumn{2}{|c|}{ Std. mean difference } & \multirow{2}{*}{\multicolumn{3}{|c|}{$\begin{array}{r}\text { Std. mean difference } \\
\mathrm{IV} \text {, random, } 95 \% \mathrm{CI}\end{array}$}} \\
\hline & Mean & SD & Total & Mean & SD & Total Weight & IV, random, $95 \% \mathrm{Cl}$ & & & \\
\hline Ersek 2008 & 5.7 & 1.9 & 101 & 6.3 & 1.8 & 123 & $-0.32(-0.59,-0.06)$ & & & \\
\hline Knittle 2015 & 79.8 & 40.4 & 40 & 92.8 & 37.7 & 36 & $-0.33(-0.78,0.12)$ & & & \\
\hline Lefort 1998 & 46.49 & 17.17 & 50 & 59.66 & 18.12 & 52 & $-0.74(-1.14,-0.34)$ & & & \\
\hline Manning 2014 & -4.7 & 21.01 & 48 & 2.6 & 22.39 & 46 & $-0.33(-0.74,0.07)$ & & & \\
\hline Nicholas 2013 & 37.01 & 8.5 & 43 & 41.88 & 8.7 & 48 & $-0.56(-0.98,-0.14)$ & 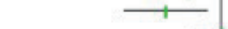 & & \\
\hline
\end{tabular}

Footnotes

(1) Education group vs, exercise control

Figure 3. Post Treatment Comparison of Self-Management Intervention Versus Control on Self-Efficacy.

Pain Intensity. Eight studies reported comparisons for pain intensity. Although the four studies that reported endpoint data showed substantial overlap of their confidence intervals, the $I^{2}$ was $55 \%$. As a sensitivity analysis showed that the heterogeneity was contributable to only one study, a meta-analysis was performed. The results indicate a statistically significant difference favouring the self-management group, SMD $=-0.28$ $[-0.56,-0.01], \mathrm{z}=2.03, \mathrm{p}=0.04$ (see Figure 4). We re-calculated this effect on an 11-point NRS scale (0-10), using the baseline standard deviation of the control group in Nicholas et al. (2013). This effect corresponds to a 0.48 difference in pain intensity, measured on a $0-10$ NRS, which is lower than the minimal clinically important difference (MCID) of 2.0 (Salaffi et al., 2004). Manning et al. (2014) reported a similar result: $\mathrm{SMD}=-0.44[-0.86,-0.02], \mathrm{z}=$ 2.05, $\mathrm{p}=$ 0.04. Burckhardt et al. (1994), Dworkin et al. (2002) and Taal et al. (1993) reported no statistically significant post-intervention differences for pain intensity between the self-management and control groups. 


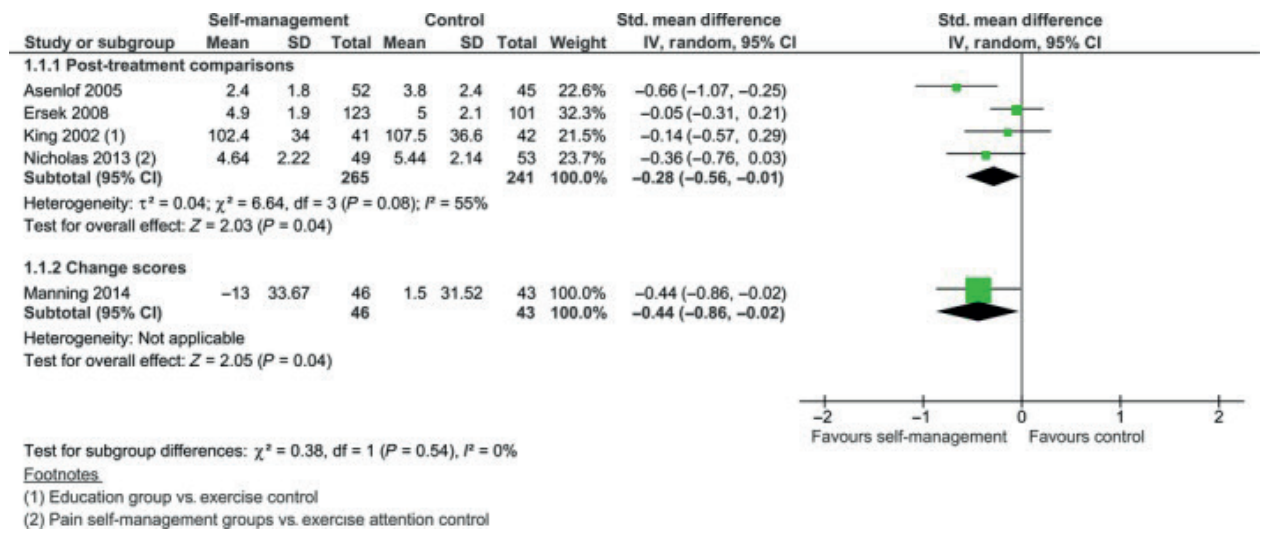

Figure 4. Post treatment comparison of self-management intervention versus control on pain intensity.

Physical Activity. Only three studies compared differences in changes of physical activity immediately post-treatment. Two studies provided sufficient information for a metaanalysis (Stuifbergen et al., 2010; Knittle et al., 2015). We pooled these study outcomes as the $I^{2}$ was $0 \%$ and there was substantial overlap in the two confidence intervals. There was no significant difference between the intervention group and the control group, $\mathrm{SMD}=0.14$ $[0.38,-0.14], \mathrm{z}=1.18, \mathrm{p}=0.24$ (see Figure 5). This is in line with Taal et al. (1993), who also did not find a difference between self-management and control groups on physical activity.

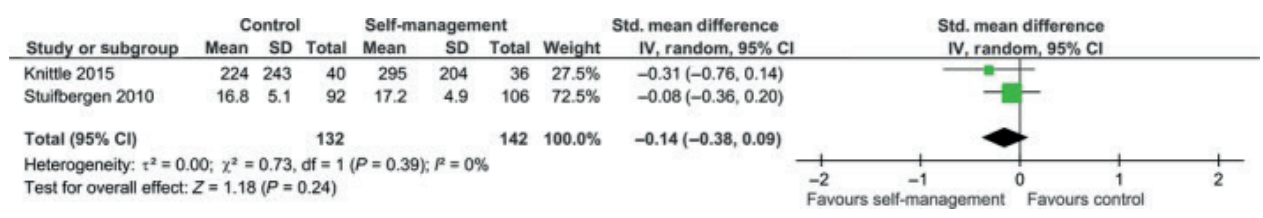

Figure 5. Post treatment comparison of self-management intervention versus control on physical activity.

Evaluation of the Evidence. The GRADE evidence plot (table 4) shows the post-intervention comparisons combined with the quality of evidence. For each outcome measure, fewer than $25 \%$ of the participants were from high risk of bias studies. The inconsistency was high for self-efficacy, due to high statistical heterogeneity compared with low overlap in confidence intervals. For the other outcome measures, the statistical heterogeneity was either limited or mainly contributable to one study. As a result of substantial variations in intervention content and outcome measures, all comparisons were downgraded for indirectness. Physical activity was the only comparison downgraded for imprecision, because the combined sample size was smaller than the optimal information size. Visual inspection of the funnel 


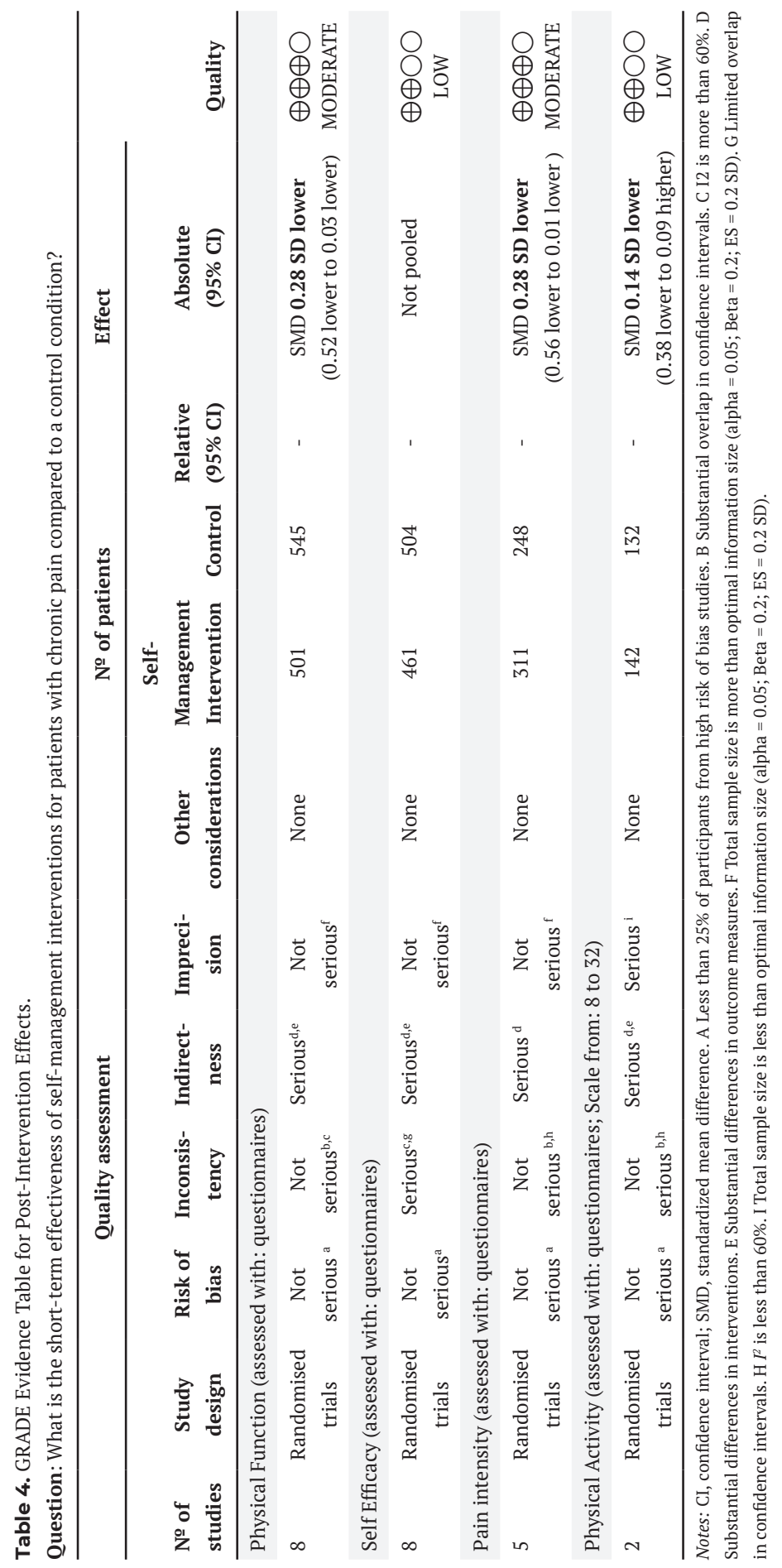


plots (see Supplement 3) did not indicate any publication bias. This resulted in the following evidence statements: For physical function and pain intensity, there is moderate quality evidence for a small but clinically insignificant effect favouring self-management. For physical activity, there is low quality evidence for no effect of self-management compared to a control group. Although we did not calculate standardized mean differences for selfefficacy, based on the range of effects, we conclude that there is low quality evidence for a trend favouring the self-management intervention. The studies that were not included in the meta-analysis showed similar results and support these conclusions.

\section{Follow-up results}

Limitations in Physical Function. Twelve out of 15 studies with follow-up data were eligible for pooling (see Figure 6). The median follow-up time of all 15 studies was 12 months. As the statistical heterogeneity was low $\left(I^{2}=0 \%\right)$, we performed a meta-analysis. The pooled effect of 11 studies with endpoint data was not statistically significant, SMD = $-0.07[-0.16,0.02], \mathrm{z}=1.60, \mathrm{p}=0.11$, and this was also the case for Manning et al. (2014), SMD $=-0.06$ [-0.47, 0.36], $\mathrm{z}=0.27, \mathrm{p}=0.78$. In addition, Hutting et al. (2015) and Taal et al. (1993) also reported no effects at follow-up. The only study that reported a long-term positive effect on physical function was Dworkin et al. (2002); at 12 months follow-up, the self-management group showed less limitation in physical function compared to control, $\mathrm{p}=0.01$.

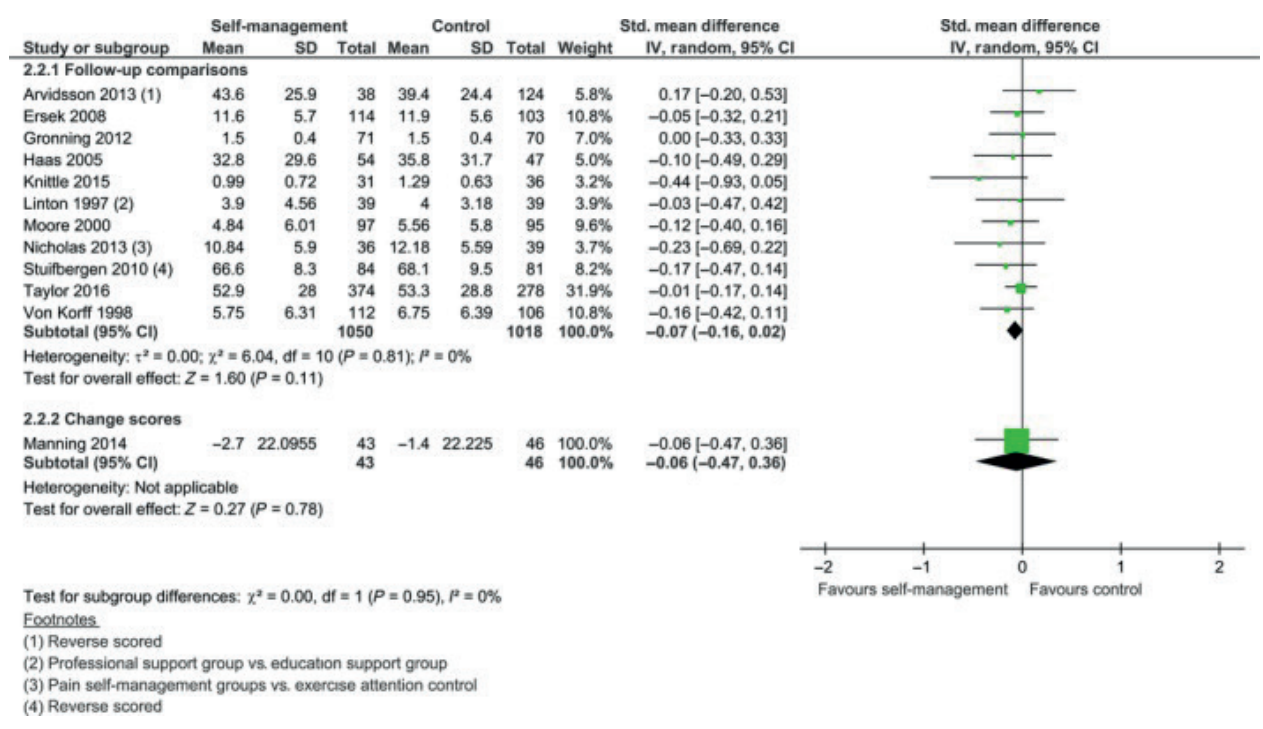

Figure 6. Follow-up Comparison of Self-Management Intervention Versus Control on Physical Function. 
Self-Efficacy. For self-efficacy, the median follow-up time was 12 months. Six of eight studies were included in the meta-analysis (see Figure 7). We found an $I^{2}$ of $0 \%$, indicating homogeneous results across studies. For five studies with endpoint data, there was a significant effect for self-efficacy at follow-up favouring the self-management group, SMD $=-0.13[-0.25,-0.02], \mathrm{z}=2.23, \mathrm{p}=0.03$. This corresponds to a difference of 1.72 points on the PSEQ (0-60), using the standard deviation of the baseline control group of Nicholas et al. (2013) as reference. As the minimal important change of the PSEQ is estimated at 5.5 points (Chiarotto et al., 2016), this mean difference is clinically insignificant. Manning et al. (2014) did not find a difference at follow-up, SMD $=-0.26[-0.68,0.16], z=1.22, p=$ 0.22 . Taal et al. (1993) reported a significant difference for self-efficacy favouring the selfmanagement group $(\mathrm{p}<0.05)$ with a positive change score of 0.17 for the self-management group and a change score of -0.06 for the control group at 13 months follow-up, whereas Hutting et al. (2015) did not find a difference at follow-up.

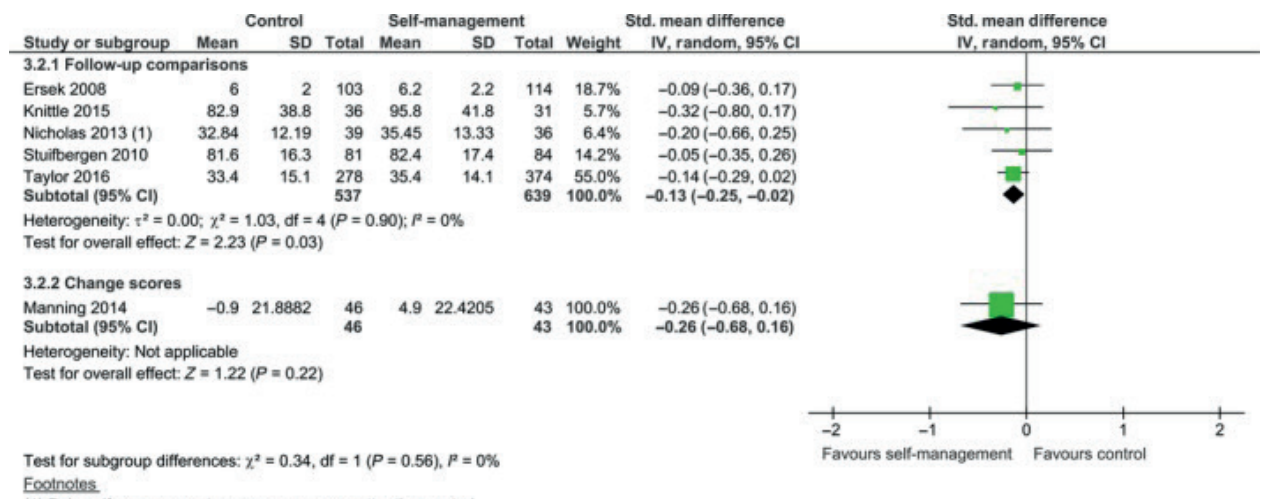

(1) Pain self-management groups vs, exercise attention control

Figure 7. Follow-up Comparison of Self-Management Intervention Versus Control on Self-Efficacy.

Pain Intensity. Ten of the 13 studies were included in the meta-analysis (see Figure 8). The median follow-up time for all 13 studies was 12 months. We decided to pool the results as the $I^{2}$ was $40 \%$ and the overlap of confidence intervals was sufficient. The pooled standardized mean difference of the nine studies with endpoint data was not statistically significant, $\mathrm{SMD}=-0.04[-0.17,0.09], \mathrm{z}=0.61, \mathrm{p}=0.54$. This was similar for Manning et al. (2014): SMD $=-0.35[-0.77,0.07], \mathrm{z}=1.62, \mathrm{p}=0.11$. Taal et al. (1993) and Hutting et al. (2015) also did not report a significant difference at follow-up, but Dworkin et al. (2002) indicated that, at 12 months follow-up, pain intensity was lower for the experimental group, compared to the control group $(\mathrm{p}=0.036)$. 


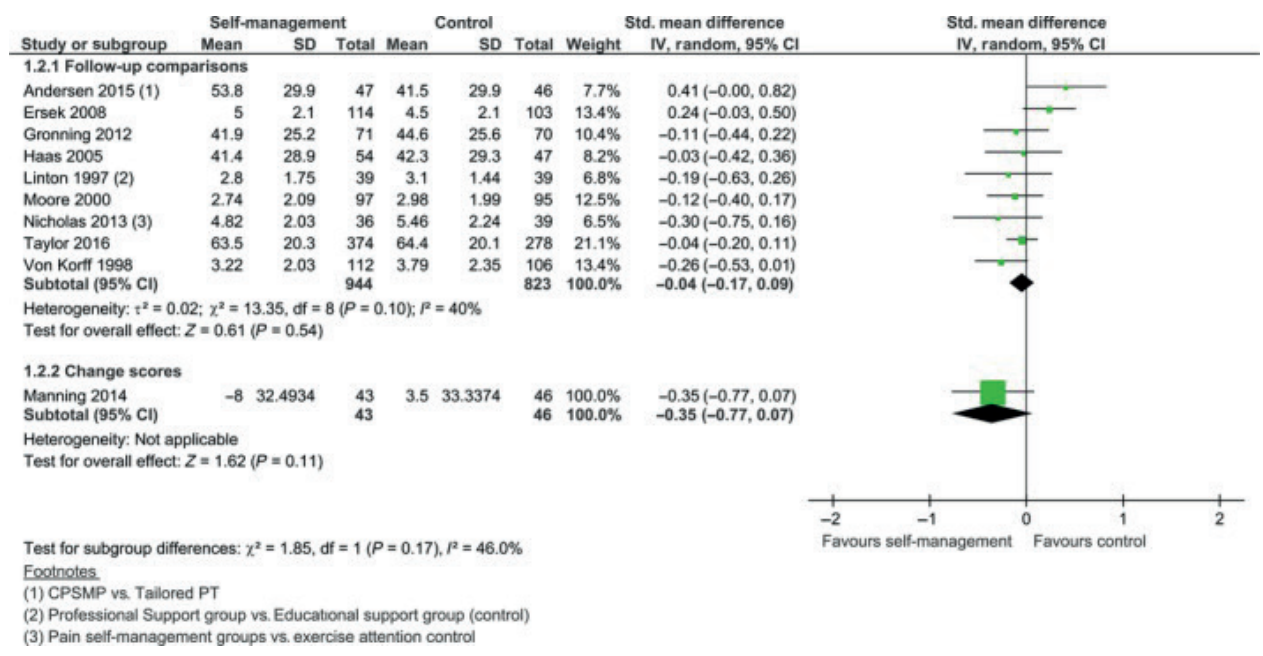

Figure 8. Follow-up Comparison of Self-Management Intervention Versus Control on Pain Intensity.

Physical Activity. Four studies provided information on follow-up time for physical activity, with a median follow-up time of 9 months. Three studies were eligible for pooling. We performed a meta-analysis as the confidence intervals largely overlapped and the $I^{2}$ was $0 \%$ (see Figure 9). There was no overall group-effect on physical activity, SMD $=0.15[-0.07$, $0.38], \mathrm{z}=1.34, \mathrm{p}=0.18$. Taal et al. (1993) also reported no differences between both groups at follow-up.

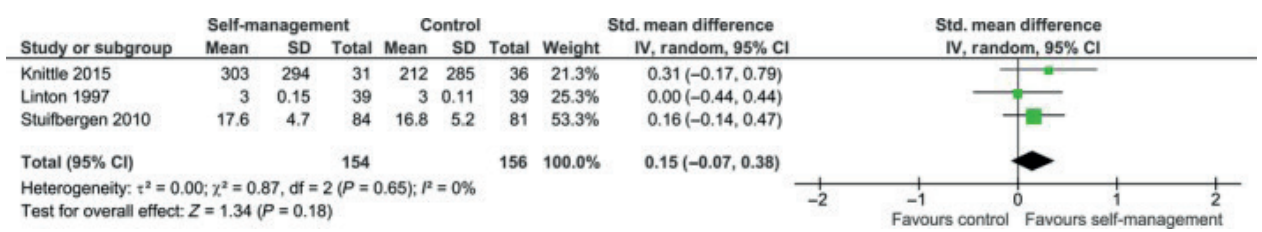

Figure 9. Follow-up Comparison of Self-Management Intervention Versus Control on Physical Activity.

Evaluation of the Evidence. The GRADE evidence plot (Table 5) shows the standardized mean differences in combination with the quality of evidence ratings. The outcomes were evaluated similarly to the post-intervention results, with the exception of a high consistency score for self-efficacy. This resulted in the following evidence statements: At six to thirteen months follow-up, there is moderate quality evidence that self-management interventions have a statistically significant, but clinically unimportant effect on selfefficacy. For pain and physical function, there is moderate quality evidence that self- 


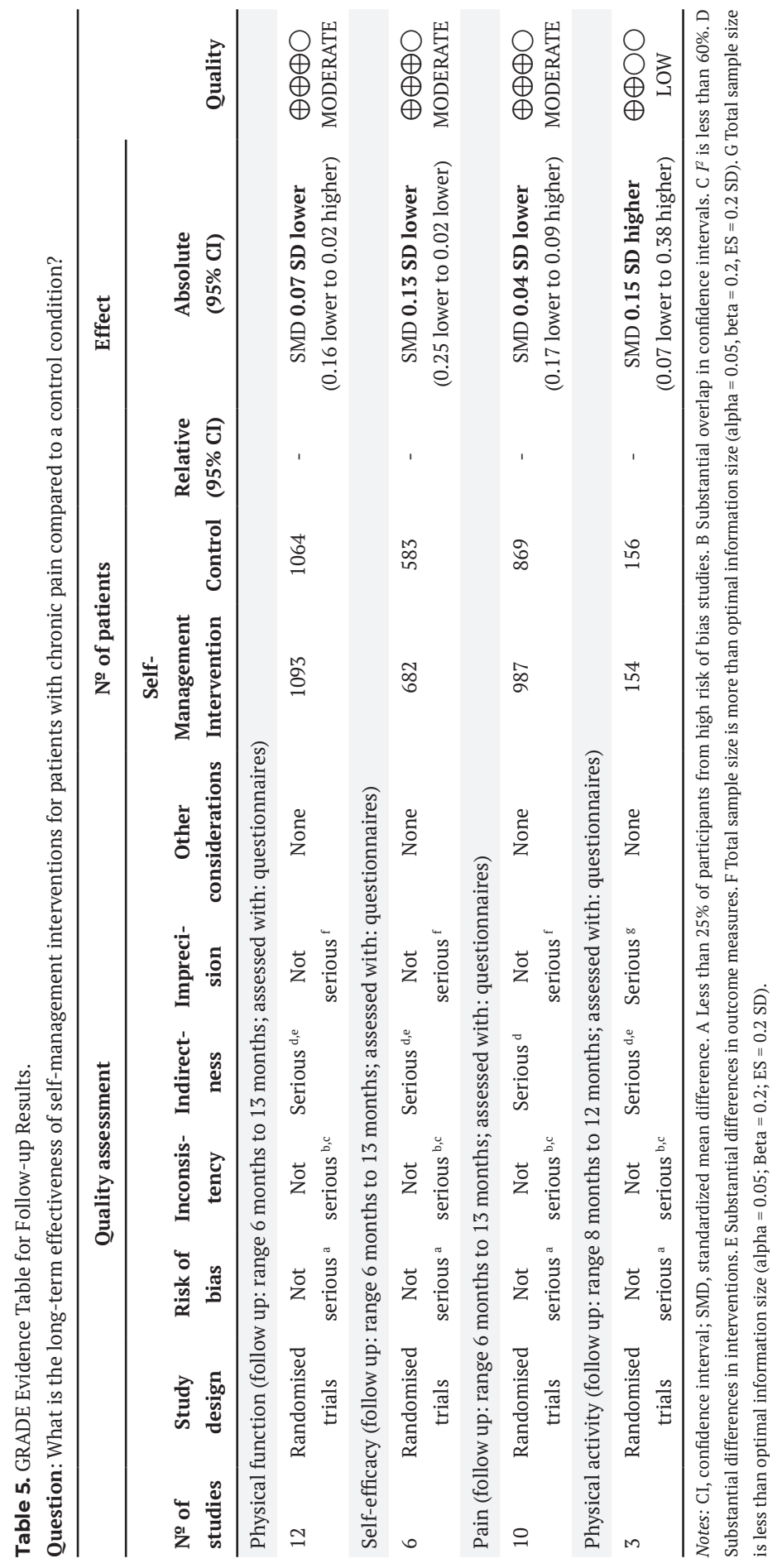


management intervention groups are not more effective than control groups. For physical activity, there is low quality evidence that self-management interventions are not more effective than control groups. The studies that could not be included in the GRADE analysis showed similar trends and corroborated these conclusions.

\section{DISCUSSION}

\section{Summary of Main Results}

The primary aim of this study was to investigate the effectiveness of self-management interventions on physical function, self-efficacy, pain intensity and physical activity for patients with chronic musculoskeletal pain. We identified 20 randomized controlled trials that compared a self-management intervention to a control group. For postintervention results, we found moderate quality evidence for a statistically significant, but clinically unimportant effect on physical function and pain intensity, both favouring the self-management group. We also found low quality evidence for a trend favouring selfmanagement interventions on self-efficacy and for no effect on physical activity. There was moderate quality evidence for a small, clinically insignificant, effect on self-efficacy at follow-up. We found moderate quality evidence for no between-group differences at follow-up for the remaining outcome measures. The results from the meta-analyses were corroborated by the studies that could not be included in the pooling. These findings indicate that self-management interventions have an only marginal benefit for patients with chronic musculoskeletal pain both in the short and long-term. Furthermore, we found a large variety in BCTs used, indicating substantial differences between interventions in how to teach self-management skills.

\section{Similarities to and Differences With Other Systematic Reviews}

We identified four related systematic reviews that show similar trends in effectiveness. Jordan et al. (2010) identified one subgroup of self-management interventions that specifically targeted patients with osteoarthritis. For both short- and long-term comparisons with control groups, the effects on clinical outcomes were inconclusive: Three studies of seven showed improvement on pain intensity; and for functional disability and quality of life, one out of five studies reported better results for the self-management group than the control group. In addition, Nolte and Osborne (2013) evaluated the outcomes of 18 self-management interventions that adopted the Stanford criteria and concluded that these interventions were only marginally effective for pain, disability and depression. Only the median effect sizes for self-efficacy, $d=0.30$ (Range: 0.05-0.72), and for knowledge, $\mathrm{d}=0.78$ (range: -0.05 to 1.11 ), were medium to large at post-intervention. Warsi et al. 
(2004) did not find a significant improvement on pain and disability associated with selfmanagement interventions for patients with arthritis. This also holds for Kroon et al. (2014), who performed a systematic review and meta-analysis to assess the effectiveness of self-management programmes in patients with osteoarthritis. They concluded that selfmanagement interventions caused small to no benefits, which is in line with the current findings. We also found two systematic reviews with contrasting findings. Du et al. (2011) studied self-management interventions for patients with chronic musculoskeletal pain and concluded that these were effective on pain intensity and disability. However, the pooled results only showed a trend in favour of self-management for patients with chronic low back pain and a statistically significant but small change in disability and pain intensity for patients with arthritis. For Du et al. (2017), the pooled comparisons (intervention vs. control) were statistically significant at all time points for patients with chronic low back pain, but the effect sizes were small (ranging from -0.20 to -0.29 for pain intensity and -0.19 to -0.28 for disability). Differences in inclusion criteria concerning the interventions could further explain the variations in outcomes.

\section{Future Directions}

Although the results of this study may not be surprising in light of the previous findings from systematic reviews, there is a large body of evidence that shows how psychological adjustment in the situation of a chronic disease may lead to favourable outcomes, such as improved well-being and adaptive lifestyle changes (Stanton et al., 2007; de Ridder et al., 2008; Kamper et al., 2015). Below we will discuss three ideas that may explain why generic self-management interventions are not as effective as expected and that could direct future research and intervention design. First, lasting behaviour change is a daunting challenge, which involves not only motivational factors such as self-efficacy and intention, but also automatic processes such as habit formation (Webb and Sheeran, 2006; Strack and Deutsch, 2004; Papies, 2016). For patients with enduring pain, these automatic factors may be of particular importance as they have coped with their pain often for several years, thereby allowing habitual routines to develop in response to pain perception. This could explain the marginal long-term effects because habits are difficult to modify, especially when interventions do not take these automatic behavioural processes into account (Papies, 2016). In order to successfully counter these habitual behaviours in interventions, Papies (2016) proposes a different approach with more emphasis on analysing and modifying these specific routines. This personalized approach differs from generic self-management interventions that provide one set of skills expected to benefit all patients. In order to capture the individual tailoring that is required in these interventions, we endorse the recommendation of Morley et al. (2013) to further explore the potential of single-case methodology. For example, experience sampling technology - where multiple (near) real- 
time self-reports of thoughts, feelings or activities can be obtained - could provide a more detailed insight in longitudinal individual response patterns to treatment (Vlaeyen et al., 2001; Maes et al., 2015).

Second, Keogh et al. (2015) attribute the limited effectiveness and large variety in content and delivery of self-management interventions to limited and inconsistent application of behaviour change theory throughout the intervention. Increased self-efficacy is often mentioned as an explanatory (mediating) factor, but it remains unclear how more confidence in the capability to live a meaningful life with pain would explain all postintervention results, including pain intensity. In particular, as we only identified a postintervention trend for self-efficacy favouring self-management interventions, other mechanisms that have not yet been identified could be responsible for the small shortterm effects on pain intensity and physical function. We believe that future research on moderators and mediators of the relationship between self-management interventions and outcome measures could provide insight in how to optimize the effectiveness of this type of intervention.

Third, despite the limited effectiveness of stand-alone generic interventions, selfmanagement skills such as problem-solving, action-planning and decision making have the potential to reinforce existing pain management treatments. Indeed, self-management is regarded as a common component in interdisciplinary pain management programmes and is expected to facilitate more active and resilient coping (McCracken and Turk, 2002; Turk et al., 2011). Future studies should investigate the interaction between selfmanagement skill training and disease-specific treatment components. This would lead to more insights on the contribution of self-management skill training to long-term effects of pain management programmes.

\section{Strengths and Limitations}

Although all included studies focused on enhancing generic self-management skills in order to improve clinical outcomes, there was a large variation on how to achieve and measure this. As a consequence, the methodological heterogeneity of the included studies negatively influenced the robustness of the outcomes. Therefore, the overall quality of evidence was downgraded for each comparison on indirectness. This also caused us to select a random-effects model, which made the pooled results difficult to interpret (Higgins and Green, 2011), even when the effect was re-expressed on the measurement scale of interest. Although this method provides an indication of the clinical importance of the effect, it cannot be regarded as a conclusive result. This is mainly because MCIDs are concerned with the effect at individual patient level rather than on mean scores at group level. However, an advantage of statistical pooling over qualitative forms of synthesis is that sample 
weights are included in the calculation of the overall effect. Visual inspection of the forest plots showed that only few individual studies reported small but statistically-significant effects, indicating that other forms of synthesis probably would have yielded similar interpretations. A second limitation is that our conclusions relate to average group effects and do not provide more detailed information on the proportion of patients that respond well to self-management interventions. Although a responder analysis is recommended (Henschke et al., 2014), very few studies provided such details. The consequence is that we were unable to explore beyond an average effect at study level.

Furthermore, we aimed to expose the various mechanisms of self-management interventions by identifying and classifying the behaviour change strategies as much as possible. This method revealed commonly used strategies (e.g. a focus on goals and planning) as well as variation in the selection of techniques to support adaptive behaviour change for patients with chronic pain. This approach opened the black box of self-management interventions to a certain extent. Although it seemed a logical next step to investigate whether specific combinations of BCTs influence the outcomes (e.g. Michie et al., 2009), we refrained from doing these analyses. Due to the generally small standardized mean differences throughout the comparisons (range SMD between studies $=-1$ to 0.41 ), we hypothesized that further exploration would not yield meaningful information.

\section{CONCLUSION}

There is moderate quality evidence that generic self-management interventions have a small clinically unimportant post-intervention effect on physical function and pain intensity. For physical activity, there is low quality evidence for no post-intervention effect and for selfefficacy, though we identified a trend favouring self-management interventions. At followup, there is moderate quality evidence for no effect of self-management interventions on physical function and pain, and low quality evidence for no effect on physical activity. In addition, we found a small but clinically unimportant long-term effect for self-management interventions on self-efficacy. Overall, these findings indicate that self-management interventions only have a marginal benefit on self-efficacy, pain intensity, physical function, and physical activity for patients with chronic musculoskeletal pain.

\section{Acknowledgements}

We acknowledge the support of Jurgen Mollema MSc from the University of Applied Sciences Utrecht with constructing the search string and performing the literature search. Issues concerning statistical analyses were discussed with Cas Kruitwagen, MSc from the Julius Center for Health Sciences and Primary Care, University Medical Center Utrecht. We 
also thank Prof. Jos Kleinen for his advice on the final draft. This research was funded by a SIA RAAK grant (2014-01-23P) and by a personal PhD grant from the University of Applied Sciences Utrecht. Proofreadingwas done by Les Hearn (les_hearn@yahoo.co.uk). 


\section{REFERENCES}

Andersen, L.N., Juul-Kristensen, B., Sorensen, T.L., Herborg, L.G., Roessler, K.K., Sogaard, K. (2015). Efficacy of tailored physical activity or chronic pain self-management programme on return to work for sicklisted citizens: A 3-month randomised controlled trial. Scand J Public Health, 43, 694-703. https://doi.org/ $10.1177 / 1403494815591687$

Andersen, L.N., Juul-Kristensen, B., Sorensen, T.L., Herborg, L.G., Roessler, K.K., Sogaard, K. (2016). Longer term follow-up on effects of tailored physical activity or chronic pain self-management programme on return-towork: A randomized controlled trial. J Rehab Med, 48, 887-892. https://doi.org/10.2340/16501977-2159

Arvidsson, S., Bergman, S., Arvidsson, B., Fridlund, B., Tingström, P. (2013). Effects of a self- care promoting problembased learning programme in people with rheumatic diseases: A randomized controlled study. J Adv Nurs, 69, 1500-1514. https://doi.org/10.1111/jan.12008

Asenlof, P., Denison, E., Lindberg, P. (2005). Individually tailored treatment targeting activity, motor behavior, and cognition reduces pain-related disability: A randomized controlled trial in patients with musculoskeletal pain. J Pain, 6, 588-603. https://doi.org/10.1016/j.jpain.2005.03.008

Barlow, J., Wright, C., Sheasby, J., Turner, A., Hainsworth, J. (2002). Self-management approaches for people with chronic conditions: A review. Patient Educ Couns, 48, 177- 187. https://doi.org/10.1016/s0738-3991(02)00032-0

Burckhardt, C.S., Mannerkorpi, K., Hedenberg, L., Bjelle, A. (1994). A randomized, controlled clinical trial of education and physical training for women with fibromyalgia. J Rheumatol, 21, 714-720.

Chiarotto, A., Vanti, C., Cedraschi, C., Ferrari, S., de Lima e Sa Resende, F., Ostelo, R.W., Pillastrini, P. (2016). Responsiveness and minimal important change of the pain self- efficacy questionnaire and short forms in patients with chronic low back pain. J Pain, 17, 707-718. https://doi.org/10.1016/j.jpain.2016.02.012

Cochrane. (2014). Review Manager (RevMan) [Computer program] (Version 5.3). Copenhagen: The Nordic Cochrane Centre.

Du, S., Yuan, C., Xiao, X., Chu, J., Qiu, Y., Qian, H. (2011). Self-management programs for chronic musculoskeletal pain conditions: A systematic review and meta-analysis. Patient Educ Couns, 85, e299-e310. https://doi. org/10.1016/j.pec.2011.02.021

Du, S., Hu, L., Dong, J., Xu, G., Chen, X. et al. (2017). Self-management program for chronic low back pain: A systematic review and meta-analysis. Patient Educ Couns, 100, 37-49. https://doi.org/10.1016/j.pec.2016.07.029

Dworkin, S.F., Huggins, K.H., Wilson, L., Mancl, L., Turner, J., Massoth, D., Leresche, L., Truelove, E. (2002). A randomized clinical trial using research diagnostic criteria for temporomandibular disorders-axis II to target clinic cases for a tailored self-care TMD treatment program. J Orofac Pain, 16, 48-63.

Eccleston, C., Fisher, E., Craig, L., Duggan, G.B., Rosser, B.A., Keogh, E. (2014). Psychological therapies (internetdelivered) for the management of chronic pain in adults. Cochrane Database of Syst Rev, 1-58. https://doi. org/10.1002/14651858.CD010152.pub2

Ersek, M., Turner, J.A., Cain, K.C., Kemp, C.A. (2008). Results of a randomized controlled trial to examine the efficacy of a chronic pain self-management group for older adults. Pain, 138, 29-40. https://doi.org/10.1016/j. pain.2007.11.003

Evidence Prime I. (2015). GRADEpro GDT: GRADEpro guideline development tool [Software]: McMaster University.

Grønnning, K., Skomsvoll, J.F., Rannestad, T., Steinsbekk, A. (2012). The effect of an educational programme consisting of group and individual arthritis education for patients with polyarthritis - a randomised controlled trial. Patient Educ Couns, 88, 113-120. https://doi.org/10.1016/j.pec.2011.12.011 
Grønning, K., Rannestad, T., Skomsvoll, J.F., Rygg, L.O., Steinsbekk, A. (2013). Long-term effects of a nurse-led group and individual patient education programme for patients with chronic inflammatory polyarthritis - a randomized controlled trial. J Clin Nurs, 23, 1005-1017. https://doi.org/10.1111/jocn.12353

Haas, M., Groupp, E., Muench, J., Kraemer, D., Brummel-Smith, K. et al. (2005). Chronic disease self-management program for low back pain in the elderly. J Manipulative Physiol Ther, 28, 228-237. https://doi.org/10.1016/j. jmpt.2005.03.010

Henschke, N., Van Enst, A., Froud, R., Ostelo, R.W.G. (2014). Responder analyses in randomised controlled trials for chronic low back pain: An overview of currently used methods. Eur Spine J, 23, 772-778. https://doi. org/10.1007/s00586-013-3155-0

Higgins, J.P.T., Green, S. (2011). Cochrane Handbook for Systematic Reviews of Interventions Version 5.1.0 The Cochrane Collaboration.

Hutting, N., Staal, J.B., Engels, J.A., Heerkens, Y.F., Detaille, S.I., Nijhuis-van der Sanden, M.W. (2015). Effect evaluation of a self-management programme for employees with complaints of the arm, neck or shoulder: A randomised controlled trial. Occup Environ Med, 72, 852-861. https://doi.org/10.1136/oemed-2015-103089

Jordan, J.L., Holden, M.A., Mason, E.E., Foster, N.E. (2010). Interventions to improve adherence to exercise for chronic musculoskeletal pain in adults (review). Cochrane Database Syst Rev, 1-64. https://doi.org/10.1002/14651858. CD005956.pub2

Kamper, S.J., Apeldoorn, A.T., Chiarotto, A., Smeets, R.J., Ostelo, R.W., Guzman, J., van Tulder, M.W. (2015). Multidisciplinary biopsychosocial rehabilitation for chronic low back pain: Cochrane systematic review and meta-analysis. BMJ, 350, h444. https://doi.org/10.1136/bmj.h444

Keogh, A., Tully, M.A., Matthews, J., Hurley, D.A. (2015). A review of behaviour change theories and techniques used in group based self-management programmes for chronic low back pain and arthritis. Man Ther, 20, 727-735. https://doi.org/10.1016/j.math.2015.03.014

King, S.J., Wessel, J., Bhambhani, Y., Sholter, D., Maksymowych, W. (2002). The effects of exercise and education, individually or combined, in women with fibromyalgia. J Rheumatol, 29, 2620-2627.

Knittle, K., De Gucht, V., Hurkmans, E., Peeters, A., Ronday, K., Maes, S., Vlieland, T.V. (2015). Targeting motivation and self-regulation to increase physical activity among patients with rheumatoid arthritis: A randomised controlled trial. Clin Rheumatol, 34, 231-238. https://doi.org/10.1007/s10067-013-2425-x

Kroon, F.P., der Van Burg, L.R., Buchbinder, R., Osborne, R.H., Johnston, R.V., Pitt, V. (2014). Self-management education programmes for osteoarthritis. Cochrane Database Syst Rev. https://doi.org/10.1002/14651858. CD008963.pub2

Lefort, S.M., Gray-Donald, K., Rowat, K.M., Jeans, M.E. (1998). Randomized controlled trial of a community-based psychoeducation program for the self-management of chronic pain. Pain, 74, 297-306. https://doi.org/10.1016/ s0304-3959(97)00190-5

Linton, S.J., Hellsing, A.L., Larsson, I. (1997). Bridging the gap: Support groups do not enhance long-term outcome in chronic back pain. Clin J Pain, 13, 221-228. https://doi.org/ 10.1097/00002508-199709000-00007

Lorig, K.R., Holman, H.R. (2003). Self-management education: History, definition, outcomes and mechanisms. Ann Behav Med, 26, 1-7. https://doi.org/ 10.1207/S15324796ABM2601_01

Maes, I.H.L., Delespaul, P.A.E.G., Peters, M.L., White, M.P., van Horn, Y., Schruers, K., Anteunis, L., Joore, M. (2015). Measuring health-related quality of life by experiences: The experience sampling method. Value Health, 18, 44-51. https://doi.org/ 10.1016/j.jval.2014.10.003

Manning, V.L., Hurley, M.V., Scott, D.L., Coker, B., Choy, E., Bearne, L.M. (2014). Education, self-management, and upper extremity exercise training in people with rheumatoid arthritis: A randomized controlled trial. Arthr Care Res, 66, 217-227. https://doi.org/10.1002/acr.22102 
McCracken, L.M., Turk, D.C. (2002). Behavioral and cognitive behavioral treatment for chronic pain. Spine (Phila Pa 1976), 27, 2564-2573. https://doi.org/10.1097/00007632-200211150-00033

Meng, K., Seekatz, B., Roband, H., Worringen, U., Vogel, H., Faller, H. (2011). Intermediate and long-term effects of a standardized back school for inpatient orthopedic rehabilitation on illness knowledge and self-management behaviors: a randomized controlled trial. Clin J Pain, 27, 248-257.https://doi.org/10.1097/AJP.0b013e3181ffbfaf

Michie, S., Abraham, C., Whittington, C., McAteer, J., Gupta, S. (2009). Effective techniques in healthy eating and physical activity interventions: A meta-regression. Health Psychol, 28, 690-701. https://doi.org/10.1037/ a0016136

Michie, S., Richardson, M., Johnston, M., Abraham, C., Francis, J. et al. (2013). The behavior change technique taxonomy (v1) of 93 hierarchically clustered techniques: Building an international consensus for the reporting of behavior change interventions. Ann Behav Med, 46, 81-95. https://doi.org/10.1007/s12160-013-9486-6

Moore, J.E., Von Korff, M., Cherkin, D., Saunders, K., Lorig, K. (2000). A randomized trial of a cognitive-behavioral program for enhancing back pain self care in a primary care setting. Pain, 88, 145-153. https://doi.org/10.1016/ S0304-3959(00)00314-6

Morley, S., Williams, A., Ecclestone, C. (2013). Examining the evidence about psychological treatments for chronic pain: Time for a paradigm shift? Pain, 154, 1929-1931. https://doi.org/10.1016/j.pain.2013.05.049

Nicholas, M.K., Asghari, A., Blyth, F.M., Wood, B.M., Murray, R. et al. (2013). Self-management intervention for chronic pain in older adults: A randomised controlled trial. Pain, 154, 824-835. https://doi.org/10.1016/j. pain.2013.02.009

Nicholas, M.K., Asghari, A., Blyth, F.M., Wood, B.M., Murray, R. et al. (2017). Long-term outcomes from training in self-management ofchronic pain in an elderly population: A randomized controlled trial. Pain, 158, 86-95. https://doi.org/ 10.1097/j.pain.0000000000000729

Nolte, S., Osborne, R.H. (2013). A systematic review of outcomes of chronic disease self- management interventions. Qual Life Res, 22, 1805-1816. https://doi.org/ 10.1007/s11136-012-0302-8

Ouzzani, M., Hammady, H., Fedorowicz, Z., Elmagarmed, A. (2016). Rayyan - a web and mobile app for systematic reviews. Syst Rev, 5, 210. https://doi.org/10.1186/s13643-016-0384-4

Papies, E.K.(2016). Health goal priming as a situated intervention tool: how to benefit from nonconscious motivational routes to health behaviour. Health Psychol Rev, 10, 408-424. https://doi.org/10.1080/17437199.2016.1183506

de Ridder, D.T.D., Geenen, R., Kuijer, R., van Middendorp, H. (2008). Psychological adjustment to chronic disease. Lancet, 372, 246-255. https://doi.org/10.1016/S0140-6736(08)61078-8

Salaffi, F., Stancati, A., Silvestri, C.A., Ciapetti, A., Grassi, W. (2004). Minimal clinically important changes in chronic musculoskeletal pain intensity measured on a numerical rating scale. Eur J Pain, 8, 283-291. https://doi. org/10.1016/j.ejpain.2003.09.004

Schünneman, H., Brozek, J., Guyatt, G., Oxman, A. (2013). GRADE Handbook for grading quality of evidence and strength of recommendations.

Schwarzer, R. (2008). Modeling health behavior change: How to predict and modify the adoption and maintenance of health behaviors. Appl Psychol, 57, 1-29. http://doi.org/10.1111/j.1464-0597.2007.00325.x

Soer, R., Reneman, M.F., Vroomen, P.C.A.J., Stegeman, P., Coppes, M.H. (2012). Responsiveness and minimal clinically important change of the pain disability index in patients with chronic back pain. Spine (Phila Pa 1976), 37, 711-715. https://doi.org/ 10.1097/BRS.0b013e31822c8a7a

Stanton, A.L., Revenson, T.A., Tennen, H. (2007). Health psychology: Psychological adjustment to chronic disease. Ann Rev Psychol, 58, 565-592. https://doi.org/ 10.1146/annurev.psych.58.110405.085615

Strack, F., Deutsch, R. (2004). Reflective and impulsive determinants of social behavior. Pers Soc Psychol Rev, 8, 220247. https://doi.org/10.1207/s15327957pspr0803_1 
Stuifbergen, A.K., Blozis, S.A., Becker, H., Phillips, L., Timmerman, G., Kullberg, V., Morrison, J. (2010). A randomized controlled trial of a wellness intervention for women with fibromyalgia syndrome. Clin Rehabil, 24, 305-318. https://doi.org/ 10.1177/0269215509343247

Sullum, J., Clark, M.M., King, T.K. (2000). Predictors of exercise relapse in a college population. J Am Coll Health, 48, 175-180. https://doi.org/ 10.1080/07448480009595693

Taal, E., Riemsma, R.P., Brus, H.L., Seydel, E.R., Rasker, J.J., Wiegman, O. (1993). Group education for patients with rheumatoid arthritis. Patient Educ Couns, 20, 177-187. https://doi.org/10.1016/0738-3991(93)90131-f

Taylor, S.J.C., Carnes, D., Homer, K., Pincus, T., Kahan, B.C. et al. (2016). Improving the self- management of chronic pain: Coping with persistent Pain, Effectiveness Research in Self-management (COPERS). NIHR Journals Library. https://doi.org/10.3310/pgfar04140

Turk, D.C., Rudy, T.E. (1991). Neglected topics in the treatment of chronic pain patients - relapse, noncompliance, and adherence enhancement. Pain, 44, 5-28. https://doi.org/ 10.1016/0304-3959(91)90142-K

Turk, D.C., Wilson, H.D., Cahana, A. (2011). Treatment of chronic noncancer pain. Lancet, 377, 2226-2235. https://doi. org/10.1016/S0140-6736(11)60402-9

Vlaeyen, J.W., de Jong, J., Geilen, M., Heuts, P.H.T.G., van Breukelen, G. (2001). Graded exposure in vivo in the treatment of pain-related fear: A replicated single-case experimental design in four patients with chronic low back pain. Behav Res Ther, 39, 151-166. https://doi.org/10.1016/s0005-7967(99)00174-6

Vlaeyen, J.W., Morley, S., Crombez, G. (2016). The experimental analysis of the interruptive, interfering, and identitydistorting effects of chronic pain. Behav Res Ther, 86, 23-34. https://doi.org/10.1016/j.brat.2016.08.016

Von Korff, M., Moore, J.E., Lorig, K.R., Cherkin, D., Saunders, K. et al. (1998). A randomized trial of a lay person-led self-management group intervention for back pain patients in primary care. Spine (Phila Pa 1976), 23, 26082615. https://doi.org/10.1097/00007632-199812010-00016

Vos, T., Flaxman, A.D., Naghavi, M., Lozano, R., Michaud, C. et al. (2012). Years lived with disability (YLDs) for 1160 sequelae of 289 diseases and injuries 1990-2010: A systematic analysis for the Global Burden of Disease Study 2010. Lancet, 380, 2163-2196. https://doi.org/10.1016/S0140-6736(12)61729-2

Warsi, A., Wang, P.S., LaValley, M.P., Avorn, J., Solomon, D. (2004). Self-management education programs in chronic disease. Arch Intern Med, 164, 1641-1649. https://doi.org/10.1001/archinte.164.15.1641

Webb, T.L., Sheeran, P. (2006). Does changing behavioral intentions engender behavior change? A meta-analysis of the experimental evidence. Psychol Bull, 132, 249-268. https://doi.org/10.1037/0033-2909.132.2.249 


\section{SUPPLEMENTARY MATERIALS}

This chapter contains the following supplementary materials

- $\quad$ Supplement 1: Search string

- $\quad$ Supplement 2: BCT profiles

- $\quad$ Supplement 3: Funnel plots

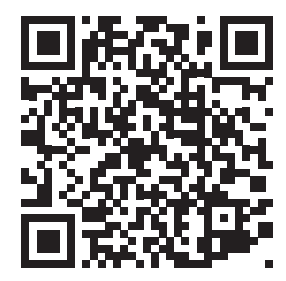

The supplementary materials can be accessed via this QR code or link https://github.com/stefanelbers/doctoral_thesis/ 



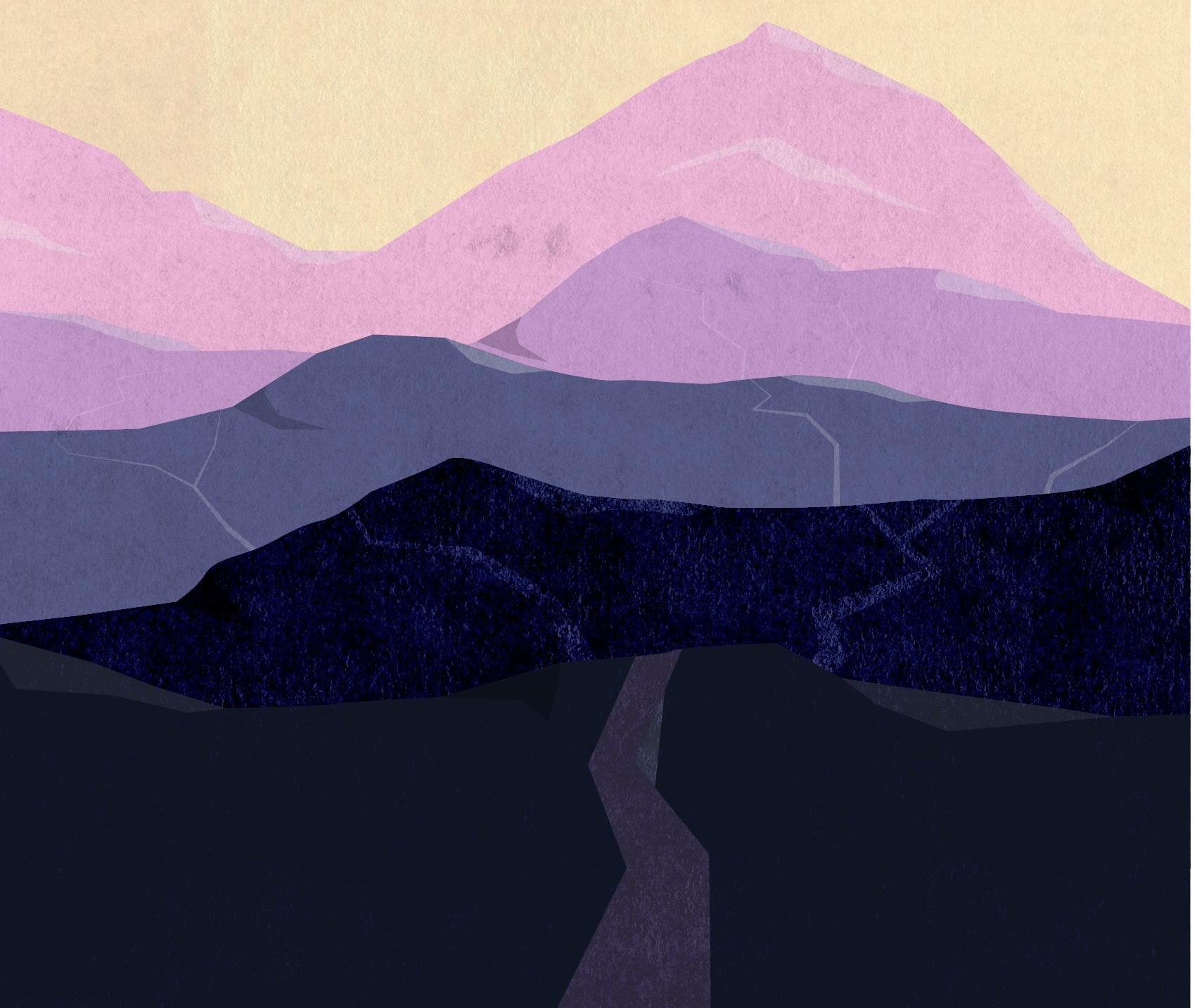




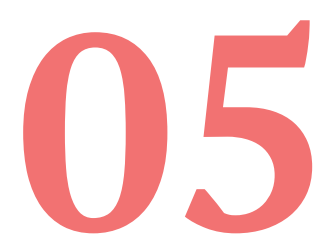

\title{
Innovation in Pain Rehabilitation Using Co-Design Methods During the Development of a Relapse Prevention Intervention:
}

\section{Case Study}

\author{
S. Elbers ${ }^{1,2}$, C. van Gessel ${ }^{3}$, R. Renes ${ }^{4}$, R. van der Lugt ${ }^{3}$, H. Wittink ${ }^{1}$, S. Hermsen ${ }^{1,5}$ \\ Journal of Medical Internet Research (2021), 23(1), e18462.
}

1. Research Group Lifestyle and Health, Utrecht University of Applied Sciences, Utrecht, The Netherlands.

2. Department of Rehabilitation Medicine, Faculty of Health, Life Sciences and Medicine, Maastricht University, Maastricht, The Netherlands.

3. Co-design Research Group, Utrecht University of Applied Sciences, Utrecht, The Netherlands.

4. Research Group Psychology of Sustainable Cities, Amsterdam University of Applied Sciences, Amsterdam, The Netherlands.

5. OnePlanet Research Center, imec The Netherlands, Wageningen, The Netherlands. 


\section{ABSTRACT}

Background: Many intervention development projects fail to bridge the gap from basic research to clinical practice. Instead of theory-based approaches to intervention development, co-design prioritizes the end users' perspective as well as continuous collaboration between stakeholders, designers, and researchers throughout the project. This alternative approach to the development of interventions is expected to promote the adaptation to existing treatment activities and to be responsive to the requirements of end users. Objective: The first objective was to provide an overview of all activities that were employed during the course of a research project to develop a relapse prevention intervention for interdisciplinary pain treatment programs. The second objective was to examine how co-design may contribute to stakeholder involvement, generation of relevant insights and ideas, and incorporation of stakeholder input into the intervention design.

Methods: We performed an embedded single case study and used the double diamond model to describe the process of intervention development. Using all available data sources, we also performed deductive content analysis to reflect on this process. Results: By critically reviewing the value and function of a co-design project with respect to idea generation, stakeholder involvement, and incorporation of stakeholder input into the intervention design, we demonstrated how co-design shaped the transition from ideas, via concepts, to a prototype for a relapse prevention intervention. Conclusions: Structural use of co-design throughout the project resulted in many different participating stakeholders and stimulating design activities. As a consequence, the majority of the components of the final prototype can be traced back to the information that stakeholders provided during the project. Although this illustrates how co-design facilitates the integration of contextual information into the intervention design, further experimental testing is required to evaluate to what extent this approach ultimately leads to improved usability as well as patient outcomes in the context of clinical practice.

Keywords: behavior change; chronic pain; co-design; intervention development; participatory design; prevention; rehabilitation; relapse. 


\section{INTRODUCTION}

Only a fraction of intervention development projects is able to bridge the translational gap from scientific research to clinical practice (Bero et al., 1998; Chambers, 2018; Czajkowski et al., 2015; de la Vega \& Miró, 2014). An important factor for this limited uptake may be that contextual factors, such as stakeholder acceptability or implementation in existing practices, receive little attention during earlier development stages (Bowen et al., 2009). For example, many intervention development guidelines emphasize the formulation of an underlying theoretical construct and subsequent experimental testing to validate each assumed causal step, but only address implementation and feasibility after the intervention development phase has been completed (Craig et al., 2008; Kok et al., 2004; Sheeran et al., 2017). Consequently, theoretically sound interventions may be discarded due to insufficient attention to crucial translational factors such as low perceived utility by patients or health care providers (HCPs), inconvenient navigation, or a discrepancy between the intervention requirements and patients' preferences (Bishop et al., 2015; Damschroder et al., 2009; Hermsen et al., 2017; Murray et al., 2010; Robert \& Macdonald, 2017; Tarquinio et al., 2015).

An opportunity to increase the emphasis on these factors is to incorporate co-design methods. Co-design not only is characterized by an incremental knowledge over multiple development cycles (Stappers \& Giaccardi, 2017) but also specifically emphasizes empathizing with each stakeholder, integrating conflicting requirements, and quickly transitioning ideas to testable prototypes. Co-design differs from other design methodologies in that it involves a range of tools and exercises to optimize collaboration between professional designers and people who are not trained in the design process, such as patients and therapists (Steen, 2008). Done rightly, co-design brings together different views, input, and competencies of people with a variety of perspectives to address a specific problem (Bradwell \& Marr, 2017; Donetto et al., 2015). As a result, this approach should increase the acceptability and integration of the intervention in existing clinical practice by accommodating relevant contextual factors that have been identified by stakeholders in the development process.

Although co-design is increasingly adopted in the development of health care interventions (e.g. Boyd et al., 2012; Donetto et al., 2015; Iedema et al., 2010; Jamin et al., 2018; Revenäs et al., 2015; Visser et al., 2005), prior studies have indicated that effective co-design is not without its challenges. For example, the process of engaging all stakeholders can be time-consuming and intensive. This can be particularly difficult in the context of health care because HCPs generally have a high workload (i.e.dema et al., 2010), and participating patients often do not directly benefit from the development projects, which could negatively 
influence their motivation and engagement. Moreover, patients, policy makers, and HCPs can experience conflicting interests during intervention development projects, because the assumed best possible care is generally limited by finite resources or specific treatment guidelines within a particular health care environment (Greger \& Hatami, 2013). Factors such as these could endanger the main principles of co-design and should be further examined in the context of health care (Boyd et al., 2012; Donetto et al., 2015).

\section{Co-Design in the Context of Chronic Pain}

In the present project, called the SOLACE project (grant number: SIA RAAK 2014-01-23), we developed a relapse prevention intervention for patients with chronic musculoskeletal pain who participate in an interdisciplinary, multimodal pain treatment program. The primary reason for adopting a co-design approach was that, despite high prevalence rates of relapse after successful treatment, there is a paucity of available research to explain relapse for this particular population (Morley, 2008; Turk \& Rudy, 1991). In these situations, a design-based approach may be particularly appropriate, because it allows for new insights to be recursively fed into future development cycles, thereby gradually increasing the knowledge base over time (Barab, 2014; Stappers \& Giaccardi, 2017). Because patients with chronic pain often experience distrust from their personal and medical environment (Toye et al., 2017), co-design may also prove effective in empowering patients to participate in the development process and to actively share their opinions and ideas (Eyles et al., 2016; Verbiest et al., 2018).

\section{Objectives}

To increase understanding of how co-design can be successfully applied in the development of interventions in the health care domain, more examples of good practice are needed (Brett et al., 2014; Donetto et al., 2015; Robert \& Macdonald, 2017). Therefore, our research question was to what extent co-design practices facilitate the translation of meaningful stakeholder experiences into the design of a health care intervention. Our first aim was to provide a detailed overview of all co-design activities that were employed during the course of an example project. Our second aim was to reflect on this overview and examine how co-design may contribute to stakeholder involvement, generation of relevant insights and ideas, and incorporation of stakeholder input into the intervention design. We hypothesized that co-design activities would facilitate the generation of relevant experiences and insights from stakeholders and stimulate their active participation during this project. Consequently, we expected that this would yield prototypes that were aligned with clinical practice and would resonate with end users. 


\section{METHODS}

\section{Design}

We performed an embedded single case study (Yin, 2003), in which we analysed and evaluated all co-design activities that were related to the development process of the SOLACE project. Throughout the study, the researchers followed a participatory action research (PAR) approach, which is characterized by active collaboration with the people of interest, rather than only researching them. PAR also emphasizes respectful cooperation between stakeholders and researchers including collective decision making and a bidirectional transfer of knowledge over multiple iterative development cycles (Foth \& Axup, 2006; Seekins \& White, 2013; White et al., 2004), which is in accordance with co-design methods (Howard \& Somerville, 2014). During each cycle, insight is acquired through action (e.g., by letting patients interact with preliminary prototypes), empowerment of stakeholders (e.g., by patient involvement in co-design sessions), and subsequent reflection (Foth \& Axup, 2006).

\section{Participants}

The SOLACE project consortium consisted of 2 interdisciplinary multimodal pain treatment centers, the Royal Dutch Society for Physical Therapy, The Dutch National Pain Patient Advocacy Organisation, and 4 research groups with a respective interest in chronic pain treatment (2 groups), co-design, and behavior change. All consortium partners assisted with the recruitment of participants when this was required at specific co-design activities, including patients and their spouses, HCPs, designers, researchers, and students. The core team was composed of 3 researchers, each from a different research group. This team was responsible for the planning and preparation of the co-design activities. To monitor overall progress, a steering committee was formed, which included representatives of all consortium partners. Ethical approval for this study was granted by the local ethics committee (Medical Research Ethics Committee Atrium 15-N-120).

\section{Materials}

Co-Design Methods. In interviews and co-creation sessions, the core team adopted various co-design methods, including generative techniques, contextual interviews, system mapping, and prototyping. These methods were adopted to facilitate stakeholder participation during key moments in the design process: generative techniques to elicit tacit knowledge and latent needs, contextual interviews to increase empathy, system mapping to develop a comprehensive overview of the acquired data, and prototyping to 
make ideas tangible and possible to experience. Co-creation sessions included multiple co-design methods and were specifically employed to empower a variety of stakeholders to participate in the design process.

Semistructured Contextual Interviews. At various time points in the project, we interviewed patients and HCPs. The interviews were performed by 2 researchers and were conducted in the everyday context of the HCPs (treatment facility) and patients (at home). To activate prior knowledge and experiences, all participants received "sensitizers"assignments that stimulated thinking about relevant topics-before the interview (see page 4 in Appendix 1) (Visser et al., 2005). During the interview, the primary interviewer explored participants' beliefs, needs, and experiences using open questions and various generative techniques. The second interviewer took notes and asked additional questions to ensure that all topics that the research team identified during preparatory sessions were covered. Data were collected by audio recording and note taking. Directly after the interview, both interviewers discussed their impressions and updated their notes.

Generative Techniques. To explore participants' ideas, needs, and values beyond their first response, various generative techniques were employed during interview and design sessions. These techniques aim to bring up "tacit knowledge" by addressing social, emotional, and functional elements related to a topic of interest (Visser et al., 2005). For example, to promote a more personal acquaintance during the interview sessions, participants were asked to introduce themselves by selecting 3 pictures from a deck of cards illustrated with ambiguous images that symbolized their personal values. The core team also used journey mapping during interviews (see page 4 in Appendix 1). This technique enabled all attendees to collaboratively construct a graphic visualization or a timeline that illustrated their experiences with interdisciplinary multimodal pain treatment (Howard, 2014; Trebble et al., 2010).

Prototyping and Provotyping. A key element of PAR is to increase insight by reflecting on actual interactions with prototypes. As Step 1.3 on page 5 of Appendix 1 illustrates, the interaction with these objects stimulated the individual to envision future possibilities or to visualize concept ideas. The process of prototyping allows participants to actively engage with objects that were based on preliminary outcomes and encompass operationalizations of the concept of interest (Sanders \& Stappers, 2014). Provotyping takes place with objects that are not directly related to the final result but are specifically designed to test a specific hypothesis or elicit a particular response (Boer \& Donovan, 2012).

System Maps and Personas. System mapping is a method for creating a visual representation of interacting variables that facilitates the understanding of complex systems 
(Jones, 2013). System maps typically include a framework of interrelated components, as well as clarifying examples of quotes and pictures. These maps can be presented in posters or cards and are useful to share data to stakeholders in an accessible way (see pages 7-9 in Appendix 1). Moreover, it provides participants with the opportunity to jointly reflect on the data and update ideas during co-creation sessions.

A specific way to represent the data as a coherent "whole" for usage throughout co-design activities is by creating personas: fictitious archetypes of users, each reflecting a distinct pattern in goals, attitudes, and behaviors based on empirical research among potential users. With personas, it is possible to highlight certain areas of tension or to facilitate discussion of important patient characteristics (Adlin et al., 2006).

Co-Creation Sessions. We used co-creation sessions at key moments during the project to discuss and reflect on the collected data, to generate new ideas, and to make decisions regarding future development directions (see page 10 in Appendix 1). A typical session would take 4 hours and involved 10-20 stakeholders. The core team prepared the sessions by formulating desired outcomes and setting up system maps to present the data. During the sessions, 2 designers operated as workshop facilitators and used various assignments (e.g., prioritizing ideas for prototype concepts) to work towards the desired outcomes in an open atmosphere where everyone was invited to actively participate. All written session data (e.g., posters, drawings, notes) were collected and discussed during core team evaluation meetings directly after the session. To maintain involvement and commitment between the sessions, the core team sent bimonthly newsletters and posted updates on the project websites.

\section{Measurements and Analysis}

Dataset. The dataset for this case study consisted of 4 different sources. To capture the results of the design methods, researchers documented each design and research activity, using observation notes, pictures, audio files, or video clips. In addition to the session documentation, researchers also organized reflective sessions directly after a codesign activity to summarize the output of co-creation sessions (e.g., notes or post-its) into system maps. These maps served both as a descriptive analysis of the data as well as for input during subsequent co-design sessions. The dataset also consisted of minutiae of steering committee meetings and a retrospective project journey. This journey was the result of a reviewing session, where researchers and members of the steering committee chronologically described and discussed critical incidents.

Data Analysis. We used a deductive content approach to identify information within the dataset that relates to our main themes: stakeholder involvement, generation of 
insights, and incorporation of stakeholder input. We defined stakeholder involvement as the commitment to participate in the development project, to collaborate with other stakeholders during design activities, and to actively participate during these sessions. Generation of insights referred to the extent by which co-design activities resulted in an increased understanding of the problem of interest that could inform subsequent development activities. Incorporation of stakeholder needs was defined as the extent by which prototypes were based on stakeholder perceptions, judgments, and evaluations from co-design activities. Furthermore, we adopted the Double Diamond model to describe the design process along 4 development stages (Design Council, 2015). The Double Diamond model contains 2 sequences of diverging and converging (Figure 1). In diverging phases, choice options and discrepancies are created; in the converging phases, these ideas are refined and considered to make design choices with respect to the prototype. In the first diamond, the "Discover" (diverging) and "Define" (converging) phases relate to acquiring insights on what to design. In the second diamond, the "Develop" and "Deliver" relate to further exploring the ideas on how to optimally design the final concepts. To illustrate how co-design contributed to the intervention development at each phase, we combined all data sources to provide both a descriptive overview and an in-depth reflection with respect to our main themes. In addition, Appendix 1 provides a chronological overview of the development process and includes examples of co-design methods, data segments, and pictures of co-design activities.

\section{RESULTS}

\section{Phase 1: "Discover"”}

Description. In the "Discover" phase, we aimed to generate a deeper understanding of factors influencing relapse after successful rehabilitation. The primary activities took place over a period of 11 months and consisted of 3 kick-off sessions, 20 stakeholder interviews (12 HCPs, 8 patients), and a student design project. In the first kick-off session, representatives from all consortium partners were present to discuss the project planning and to decide how co-design would be implemented throughout the project. Representatives also participated in co-creation by using their professional and personal experiences to formulate initial ideas on relapse (see page 3 of Appendix 1). These activities were repeated during introductory visits by the core team at the 2 participating pain treatment centers. During these visits, the core team also observed multiple treatment sessions and were given a detailed explanation about dose and content of the included treatment modalities. Subsequently, 20 semistructured interviews of approximately 1 hour were conducted and transcribed (see page 4 in Appendix 1). During the final activity of 


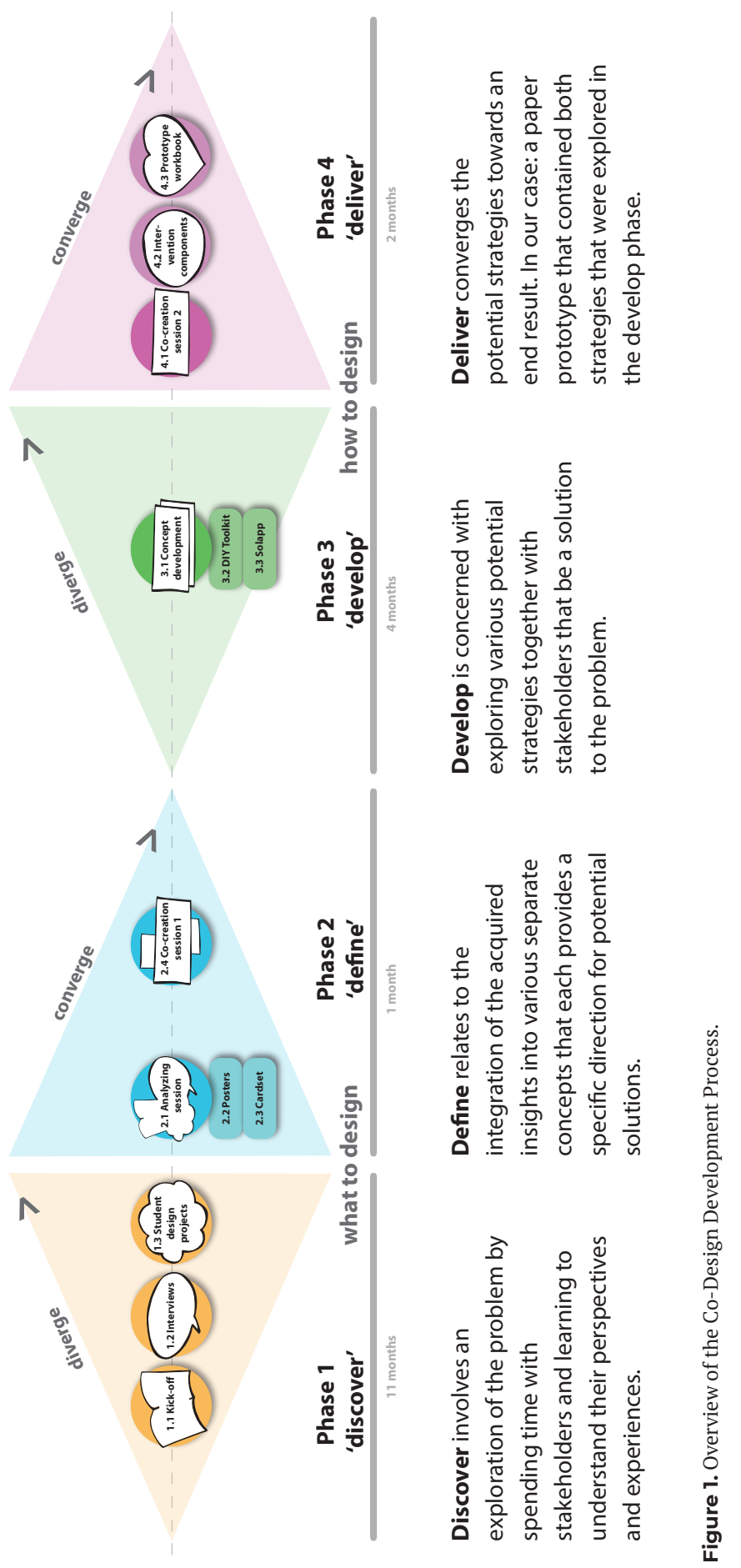


phase 1, 60 students divided over 16 teams formulated hypotheses based on the previously collected data and designed provotypes to test their ideas with both healthy participants and patients with chronic pain (see page 5 of Appendix 1). At the start of each week, they updated their provotypes based on the received feedback. During the final project session, all teams presented their final provotypes as well as their collected insights to members of the consortium.

Reflection. In phase 1, we were able to create a large qualitative dataset. This dataset not only contained experiences and ideas of stakeholders but also included specific feedback in response to multiple provotypes on a wide array of topics. The consecutive planning of the 3 key activities enabled us to iteratively expand our insights on relapse after pain treatment: Interviews were prepared by using the insights from the kick-off sessions, and the student teams could build upon the preliminary analysis of the available interview data. The participating stakeholders responded positively to the co-design approach and cooperated actively during the sessions and interviews. Despite their inexperience with co-design, the sessions were considered accessible, pleasant, and relevant. However, medical ethical screening procedures and personnel deployment planning limited the possibility for last-minute requests or invitations for including HCPs and patients. The obtained dataset of patient and HCP responses also contributed to a deeper understanding of relevant factors related to relapse, which provided a solid base for further intervention development. For example, the interviews revealed important contextual information such as a "feelings of emptiness after treatment," "difficulties sharing treatment experiences with friends and family," and "the different context between the rehabilitation center and the personal environment."

\section{Phase 2: "Define"}

Description. The "Define" phase lasted for 1 month and started with thematically organizing the interviews by means of open coding by the core team (see page 6 of Appendix 1) (Braun \& Clarke, 2006; Gale et al., 2013). This resulted in 8 main themes and 45 subthemes of factors associated with relapse after successful treatment (see page 8 in Appendix 1). To facilitate subsequent co-design activities, the themes were rephrased as questions, plotted on posters, and illustrated with exemplary quotes and figures (see page 8 in Appendix 1 for an example). In addition, the core team developed a set of 74 stimulus cards that were designed to facilitate the discussion of specific insights or principles (Coughlan et al., 2007): 36 cards contained insights from the student project, 15 cards contained relevant theory on behavior regulation, and 23 cards contained theory related to chronic pain treatment (see page 9 in Appendix 1). Subsequently, patients (4), HCPs (4), researchers (9), designers (6), and students (3) were invited for a co-creation session (see page 10 in Appendix 1). During the first assignment, participants were asked to examine 
the posters and extend them with their own ideas or with stimulus cards. This resulted in 121 notes and 42 cards that were added to the posters. In the second assignment, subgroups were made of participants with varying backgrounds. Each group was instructed to select 1 theme and use the available information to develop an intervention concept. A professional draftsman supported the session by immediately visualizing intervention ideas. The final part of the session consisted of a plenary session where all 5 concepts were presented. During the subsequent discussion, the concepts were compared, and various overarching topics emerged, including "maintaining the positive development after treatment" and "reflection and self-monitoring." In a subsequent meeting, the steering committee merged these overarching topics into 2 concept ideas: positive reinforcement and direct feedback. The "Define" phase concluded with a design briefing, where the core team commissioned 3 students to develop these ideas into tangible rudimentary prototypes as part of their graduation project.

Reflection. The final system map that included both posters and the card set provided a complete overview of the collected data. This presentation form stimulated participants to combine various insights to develop concept interventions. With respect to stakeholder involvement, the number of patients and HCPs was lower than originally planned. The duration of the session and traveling distance required participants to block a full day, which turned out to be difficult to organize. In line with our findings in phase 1 , the codesign methods successfully engaged nonexperts in the design process. The assignment to create concept intervention ideas was concrete and tangible. The resulting 5 concepts were associated with earlier identified patient needs, were grounded in contextual information, and contained relevant insights on relapse prevention. For example, one concept idea focused on monitoring and recognizing early signals of relapse, which was based on stimulus cards (e.g., a research insight related to difficulties in unbiased self-monitoring of behavior), interview data (e.g., a quote from HCP on the possibility of daily feedback via eHealth), and newly added notes (e.g., patient feedback should always be related to patientspecific goals). However, only a fraction of the possible combinations of cards and system maps was explored during this session. Limited time and resources prevented organizing additional sessions to cross-validate the results and achieve saturation.

\section{Phase 3: "Develop"}

Description. During the 4 months of the "Develop" phase, students held 5 focus groups to regularly test their ideas with patients and HCPs (see page 11 in Appendix 1). For example, by discussing the role of personal values within the treatment program, the students found supporting evidence that these values were strongly related to treatment goals, which subsequently guided the operationalization of the valued-based action plan in one of the rudimentary prototypes. Based on stakeholder feedback and weekly evaluation 
sessions with the core team, the students worked towards a final rudimentary prototype. One student focused on the transfer of important treatment insights to each patients' personal context. She developed a toolbox that contained various methods to capture and store therapy insights in order to facilitate retrieval in a relevant personal context (e.g., using a personal picture as memory cue for an important moment during treatment). The other 2 students focused on the generation of valued-based goals and the formulation of action plans for each consecutive step towards the goal. Their final rudimentary prototype consisted of a mockup mobile app, allowing participants to browse through all steps that were required to formulate and plan a valued-based goal. In Appendix 1, pages 12-13 show the final versions of these rudimentary prototypes.

Reflection. This phase was characterized by a shift from "what" to "how" to design. Accordingly, presentation form, usability, and implementation into existing treatment practice became increasingly relevant. To engage stakeholders, the students visited the treatment centers on multiple occasions. In contrast to other phases, the patients and HCPs could provide feedback on ideas, but were not involved in the decision-making process regarding the final design of the rudimentary prototype, which potentially influenced their commitment. Moreover, their reduced involvement in this phase resulted in limited information regarding the applicability of the rudimentary prototypes in clinical practice.

\section{Phase 4: "Deliver"}

Description. In the final phase, the core team merged both rudimentary prototypes into one final prototype intervention over a period of 2 months. To do so, the core team organized a final co-creation session, where the students presented their concepts. The aim of this session was to receive final feedback on the potential value and function of both rudimentary prototypes and to formulate a recommendation to the steering committee with respect to the final prototype design. To facilitate this process, stakeholders $(n=14)$ were instructed to reflect on the concepts by taking various patient perspectives into account. For this purpose, 4 personas were created with variation on 2 characteristics that were often discussed during previous patient interviews. Each persona had either a high or low level of social support and a high or low tendency to protect personal boundaries. In Appendix 1, page 14 depicts the discussions between stakeholders as well as the poster that explained the 4 personas. The final conclusion was that both rudimentary prototypes had potential as supportive treatment modalities to prevent relapse after successful treatment. Furthermore, future testing and development should primarily focus on optimizing the active treatment components and calibrating the intervention to the treatment program.

Based on this advice, the steering committee decided to merge both rudimentary prototypes into 1 prototype workbook. The core team composed a list of individual intervention 
components from each rudimentary prototype (e.g., a prompt to set calendar reminders after a goal-setting procedure) and coded these according to the Behaviour Change Technique Taxonomy V1 (see page 15 in Appendix 1) (Michie et al., 2013). Subsequently, they determined how to transfer the components to a workbook version and performed literature searches to find ideas for optimizing the effectiveness of each component. For example, to assist the formulation of personal values, various value generation procedures were found (e.g. Chase et al., 2013) and integrated into the prototype. In addition, the core team checked to which extent the list of intervention components corresponded with the themes of the interview dataset. Of the 19 intervention components, 17 components were related to one or more themes from the dataset, and 27 of the 45 themes were related to one or more intervention components. For example, 4 components in the goal-setting intervention, including specific probing questions to help formulate meaningful values, were associated with the theme "remembering important goals and values after treatment." A designer, a text editor, and 3 HCPs provided feedback with the conversion to a paper workbook intervention and respectively focused on the design, readability, and appropriate terminology. In Appendix 1, page 16 shows examples of the 2 included strategies: the value-based goal forms (b and c) and the Insight Cards (d).

Reflection. Previous difficulties with recruiting sufficient patients for co-creation sessions caused us to search for alternative ways to include their viewpoint. The personas proved a useful method to incorporate various patient perspectives by proxy during the evaluation of the rudimentary prototypes. Furthermore, the validation check indicated that the majority of the intervention components could be traced back to the original stakeholder themes from the interventions in the "Discover" phase and vice versa. This illustrates that stakeholder input has been incorporated in the design. However, the decision to combine both prototype ideas into one intervention was unexpected, which resulted in last-minute planning and consequently in limited stakeholder involvement during the design of the workbook. This may threaten the usability of this prototype in clinical practice.

\section{DISCUSSION}

\section{Principal Findings}

The primary aim of this study was to reflect on the value and function of co-design methodology during the development of an intervention that prevents relapse after successful pain treatment. In the analysis, we focused on idea generation, stakeholder involvement, and the incorporation of stakeholder input within the development process. Overall, the generative techniques that were employed supported patients and HCPs with sharing their perspectives on pain treatment and relapse, which was in line with 
our hypothesis. Moreover, the techniques steered the conversations beyond stakeholders' primary responses, often resulting in a detailed account of their personal experiences with the treatment program and of their attempts to integrate treatment insights into their personal environment. In addition, system maps, personas, and prototypes enabled nonexperts to actively participate in design activities. A possible explanation for the successful engagement of stakeholders during the project is that experienced co-designers constantly translated hypotheses and abstract ideas into provotypes or prototypes. This method is particularly useful to provoke user reactions or to rapidly visualize an idea, which evokes interactions with an actual object rather than reflections on past experiences of hypothetical situations (Sanders \& Stappers, 2014). In addition, the used co-design materials helped to transform each location where co-design activities took place (e.g., treatment facility or patient home) into a workshop environment that stimulated active participating and emphasized equality between all participants. This is especially important for health care settings, where conventional power relationships between patients and HCPs threaten effective cooperation during design activities (Boyd et al., 2012; Donetto et al., 2015).

With respect to stakeholder involvement, many different patients, HCPs, researchers, students, and designers participated during the study, which was also in line with our hypothesis. The stakeholder interactions mostly consisted of independent design activities that required low commitment and little effort. In contrast, the members of the core team remained active throughout the project, which increasingly created an imbalance in knowledge and involvement between the core team and other participants in co-design activities. This may explain why the role of the stakeholders gradually shifted from "user as partner"-where all participants within the sessions contributed as equals in the design activities-towards "users as subject"-where participants mainly provided expert opinions or performed delimited tasks (e.g., usability testing) (Sanders \& Stappers, 2008). Consequently, the concepts underlying the intervention have been thoroughly grounded in stakeholder input and expertise, but the applicability of the current workbook operationalization within the treatment programs requires further testing to examine whether the current strategies fit patient preferences and can be integrated in treatment programs in the form of the current prototype.

This project shows similarities to the experience-based co-design (EBCD) approach, which aims to improve health care services by actively involving stakeholders to collect knowledge and experiences, to set priorities, and to develop solutions (Donetto et al., 2015; Robert et al., 2015). Although this project did not follow the 6 stages of EBCD, the overall objectives as well as the systematic partnership with patients, HCPs, designers, and researchers are alike. A notable difference was the focus within this project on actual prototype development 
throughout all phases, which promoted a solution-focused orientation for the participants. Alternatively, in EBCD, more emphasis is placed on ensuring that the collected patient experiences are received and understood by other stakeholders (e.g., by showing a film of patient interview segments that reflect key themes), before continuing to developing improvements (Donetto et al., 2015). These differences illustrate the versatility of codesign and its potential to adapt to different design environments.

\section{Strengths and Limitations}

The extensive documentation of the co-design activities allowed for a detailed reconstruction of the development process. Furthermore, during co-creation sessions, steering committee meetings, and the construction of the retrospective journey, representatives from all research groups were present, which resulted in a continuous integration of various perspectives during the project. However, we did not film or record any of the co-creation sessions. Although analyzing audio or video would have been time consuming, it would have provided further possibilities to observe stakeholder discussions during design activities and to include additional insights that we did not record.

During the project, we experienced a tradeoff between validating the outcomes of co-design activities and analyzing the results for the next iteration. For example, an additional cocreation session during the "Define" phase with different stakeholders could have crossvalidated the outcomes of the initial session. However, given limited resources, this would have resulted in fewer development iterations in the remaining period. A key argument in favor of more iterations is that quickly integrating stakeholder input into subsequent sessions directly visualizes the value of their input (Hinchcliff et al., 2014). However, a tendency towards more iterations increases the uncertainty to what extent the outcomes of this project can be generalized to the population (Seekins \& White, 2013).

\section{Future Recommendations}

This study adds to the increasing number of initiatives that use co-design to structurally integrate contextual factors into the development of health care interventions (e.g. Jamin et al., 2018; O’Brien et al., 2016; Raynor et al., 2020; Revenäs et al., 2015), which help bridge the gap from development to actual implementation (Murray et al., 2010; Robert \& Macdonald, 2017). When using co-design, it is important to relate the findings of the process to existing theories and treatments, for instance by using the behavior change technique taxonomy (Michie et al., 2013; Verbiest et al., 2019). This strengthens the codesign approach by combining stakeholder evaluations with existing theory. Importantly, further integration between co-design and theory-driven approach only becomes possible when using rigorous testing to evaluate the outcomes of the co-design process (Robert \& 
Macdonald, 2017) . Consequently, an important next step in answering the question about whether co-design helps inform the development of health interventions will involve more examples of development projects. In these examples, co-design-based interventions are ideally subjected to experimental testing. Furthermore, we believe that future co-design projects in the health care domain should include detailed planning of activities and take lengthy medical ethical approval procedures into account (Hinchcliff et al., 2014).

\section{Conclusions}

To acquire a better understanding of how co-design may benefit the development of interventions in the health care domain, examples of good practice are necessary. In this article, we presented such an example. By critically reviewing the value and function of a co-design project with respect to idea generation, stakeholder involvement, and incorporation of stakeholder input into the development process, we demonstrated how co-design contributed to the transition from ideas, via concepts, to prototypes.

\section{Acknowledgments}

We thank the researchers Jan Pool, Manon Beetstra-Huszar, and Roos Tigchelaar for their continuous support with collecting and analyzing the data. We also thank Rianne Schaekens, Deloryan Hommers, and Yasmijn Baas for their assistance in the development phase during their graduation projects.

\section{Conflicts of Interest}

None declared.

\section{Abbreviations}

EBCD: experience-based co-design; HCP: health care provider; PAR: participatory action research. 


\section{REFERENCES}

Adlin, T., Pruitt, J., Goodwin, K., Hynes, C., McGrane, K., Rosenstein, A., \& Muller, M. J. (2006). Putting personas to work. In: CHI'06 Extended Abstracts on Human Factors in Computing Systems (pp 13-16). New York: Association for Computing Machinery.

Barab, S. A. (2014). Design-based research: A methodological toolkit for engineering change. In The Cambridge Handbook of the Learning Sciences, Second Edition (pp. 151-170). Cambridge University Press.

Bero, L. A., Grilli, R., Grimshaw, J. M., Harvey, E., Oxman, A. D., \& Thomson, M. A. (1998). Closing the gap between research and practice: an overview of systematic reviews of interventions to promote the implementation of research findings. BMJ, 317(7156), 465-468. https://doi.org/10.1136/bmj.317.7156.465

Bishop, F. L., Fenge-Davies, A. L., Kirby, S., Geraghty, A. W. J. P., \& health. (2015). Context effects and behaviour change techniques in randomised trials: a systematic review using the example of trials to increase adherence to physical activity in musculoskeletal pain. Psychol Health, 30(1), 104-121. https://doi.org/10.1080/08870446 .2014 .953529

Boer, L., \& Donovan, J. (2012). Provotypes for participatory innovation. In: DIS'12: Proceedings of the designing interactive systems conference ( $p p$ 388-397). New York: Association for Computing Machinery.

Bowen, D. J., Kreuter, M., Spring, B., Cofta-Woerpel, L., Linnan, L., Weiner, D., Bakken, S., Kaplan, C. P., Squiers, L., \& Fabrizio, C. J. A. j. o. p. m. (2009). How we design feasibility studies. Am J Prev Med, 36(5), 452-457. https://doi. org/ 10.1016/j.amepre.2009.02.002

Boyd, H., McKernon, S., Mullin, B., \& Old, A. (2012). Improving healthcare through the use of co-design. $N Z$ Med J, 125(1357), 4-15.

Bradwell, P., \& Marr, S. (2017). Making the most of collaboration: an international survey of public service co-design. Annual Review of Policy Design, 5(1), 1-27. https://www.demos.co.uk/files/CollabWeb.pdf [accessed 2020-12-15]

Braun, V., \& Clarke, V. (2006). Using thematic analysis in psychology. Qual Res Psychol, 3(2), 77-101. https://doi. org/10.1191/1478088706qp063oa

Brett, J., Staniszewska, S., Mockford, C., Herron-Marx, S., Hughes, J., Tysall, C., \& Suleman, R. (2014). Mapping the impact of patient and public involvement on health and social care research: a systematic review. Health Expect, 17(5), 637-650. https://doi.org/10.1111/j.1369-7625.2012.00795.x.

Chambers, C. T. (2018). From evidence to influence: Dissemination and implementation of scientific knowledge for improved pain research and management.Pain, 159, S56-S64.https://doi.org/10.1097/j.pain.0000000000001327

Chase, J. A., Houmanfar, R., Hayes, S. C., Ward, T. A., Vilardaga, J. P., \& Follette, V. (2013). Values are not just goals: Online ACT-based values training adds to goal setting in improving undergraduate college student performance. J Contextual Behav Sci, 2(3-4), 79-84. https://doi.org/10.1016/j.jcbs.2013.08.002

Coughlan, P., Suri, J. F., \& Canales, K. (2007). Prototypes as (design) tools for behavioral and organizational change: A design-based approach to help organizations change work behaviors. J appl behav sci, 43(1), 122-134. https:// doi.org/10.1177/0021886306297722

Craig, P., Dieppe, P., Macintyre, S., Michie, S., Nazareth, I., \& Petticrew, M. (2008). Developing and evaluating complex interventions: the new Medical Research Council guidance. BMJ, 337, 979-983. https://doi.org/10.1136/bmj. a1655.

Czajkowski, S. M., Powell, L. H., Adler, N., Naar-King, S., Reynolds, K. D., Hunter, C. M., Laraia, B., Olster, D. H., Perna, F. M., \& Peterson, J. C. (2015). From ideas to efficacy: The ORBIT model for developing behavioral treatments for chronic diseases. Health Psychol, 34(10), 971-982. https://doi.org/10.1037/hea0000161 
Damschroder, L. J., Aron, D. C., Keith, R. E., Kirsh, S. R., Alexander, J. A., \& Lowery, J. C. (2009). Fostering implementation of health services research findings into practice: a consolidated framework for advancing implementation science. Implement Sci, 4(1), 50. https://doi.org/10.1186/1748-5908-4-50

de la Vega, R., \& Miró, J. (2014). mHealth: a strategic field without a solid scientific soul. a systematic review of painrelated apps. PloS one, 9(7), e101312. https://doi.org/ 10.1371/journal.pone.0101312

Design Council. (2015). The Design Process: What is the Double Diamond? https://www.designcouncil.org.uk/ news-opinion/design-process-what-double-diamond. (Archived by WebCite at http://www.webcitation. org/74d5rk9mE)

Donetto, S., Pierri, P., Tsianakas, V., \& Robert, G. (2015). Experience-based co-design and healthcare improvement: realizing participatory design in the public sector. Design J, 18(2), 227-248. https://doi.org/10.2752/17563061 $5 \times 14212498964312$

Eyles, H., Jull, A., Dobson, R., Firestone, R., Whittaker, R., Te Morenga, L., Goodwin, D., \& Mhurchu, C. N. (2016). Codesign of mHealth delivered interventions: a systematic review to assess key methods and processes. Curr Nutr Rep, 5(3), 160-167. https://doi.org/10.1007/s13668-016-0165-7

Foth, M. \& Axup, J. (2006) Participatory Design and Action Research: Identical Twins or Synergetic Pair?. In Jacucci, G., Kensing, F., Wagner, I., \& Blomberg, J. (Eds.) Proceedings Participatory Design Conference 2006: Expanding Boundaries in Design (pp. 93-96), Trento, Italy.

Gale, N. K., Heath, G., Cameron, E., Rashid, S., \& Redwood, S. (2013). Using the framework method for the analysis of qualitative data in multi-disciplinary health research. BMC Med Res Methodol, 13(1), 117. https://doi. org/10.1186/1471-2288-13-117

Greger, S., \& Hatami, Z. (2013). Reducing social distance through design. In Keinonen, T., Vaajakallio, K., \& Honkonen, J. (Eds.), Designing for wellbeing (pp. 125-143). Aalto: Arts Books.

Hermsen, S., Moons, J., Kerkhof, P., Wiekens, C., \& De Groot, M. J. J. (2017). Determinants for sustained use of an activity tracker: observational study. JMIR Mhealth Uhealth, 5(10), e164. https://doi.org/10.2196/mhealth.7311

Hinchcliff, R., Greenfield, D., \& Braithwaite, J. (2014). Is it worth engaging in multi-stakeholder health services research collaborations? Reflections on key benefits, challenges and enabling mechanisms. International Journal for Quality in Health Care, 26(2), 124-128. https://doi.org/10.1093/intqhc/mzu009

Howard, T. (2014). Journey mapping: A brief overview. Commun Des Q Rev, 2(3), 10-13. https://doi. org/10.1145/2644448.2644451

Howard, Z., \& Somerville, M. M. (2014). A comparative study of two design charrettes: implications for codesign and participatory action research. CoDesign, 10(1), 46-62. https://doi.org/10.1080/15710882.2014.881883

Iedema, R., Merrick, E., Piper, D., Britton, K., Gray, J., Verma, R., \& Manning, N. (2010). Codesigning as a discursive practice in emergency health services: the architecture of deliberation. J appl behav sci, 46(1), 73-91. https:// doi.org/10.1177/0021886309357544

Jamin, G., Luyten, T., Delsing, R., \& Braun, S. (2018). The process of co-creating the interface for VENSTER, an interactive artwork for nursing home residents with dementia. Disabil Rehabil Assist Technol, 13(8), 809-818. https://doi.org/10.1080/17483107.2017.1385102

Jones, P. (2013). Design for care: Innovating healthcare experience. New York: Rosenfeld Media.

Kok, G., Schaalma, H., Ruiter, R. A., Van Empelen, P., \& Brug, J. (2004). Intervention mapping: protocol for applying health psychology theory to prevention programmes. J Health Psychol, 9(1), 85-98. https://doi. org/10.1177/1359105304038379

Michie, S., Richardson, M., Johnston, M., Abraham, C., Francis, J., Hardeman, W., Eccles, M. P., Cane, J., \& Wood, C. E. (2013). The behavior change technique taxonomy (v1) of 93 hierarchically clustered techniques: building an international consensus for the reporting of behavior change interventions. Ann Behav Med, 46(1), 81-95. https://doi.org/10.1007/s12160-013-9486-6 
Morley, S. (2008). Relapse prevention: still neglected after all these years. Pain, 134(3), 239-240. https://doi. org/10.1016/j.pain.2007.12.004

Murray, E., Treweek, S., Pope, C., MacFarlane, A., Ballini, L., Dowrick, C., Finch, T., Kennedy, A., Mair, F., \& O'Donnell, C. (2010). Normalisation process theory: a framework for developing, evaluating and implementing complex interventions. BMC Med, 8(1), 1-11. https://doi.org/10.1186/1741-7015-8-63

O’Brien, N., Heaven, B., Teal, G., Evans, E. H., Cleland, C., Moffatt, S., Sniehotta, F. F., White, M., Mathers, J. C., \& Moynihan, P. (2016). Integrating evidence from systematic reviews, qualitative research, and expert knowledge using co-design techniques to develop a web-based intervention for people in the retirement transition. J Med Int Res, 18(8). https://doi.org/10.2196/jmir.5790

Raynor, D. K., Ismail, H., Blenkinsopp, A., Fylan, B., Armitage, G., \& Silcock, J. (2020). Experience-based co-designAdapting the method for a researcher-initiated study in a multi-site setting. Health Expect, 23(3), 562-570. https://doi.org/10.1111/hex.13028

Revenäs, Å., Martin, C., Opava, C. H., Brusewitz, M., Keller, C., \& Åsenlöf, P. (2015). A mobile Internet service for selfmanagement of physical activity in people with rheumatoid arthritis: challenges in advancing the co-design process during the requirements specification phase. JMIR Res Protoc, 4(3), e111. https://doi.org/10.2196/ resprot. 4824

Robert, G., \& Macdonald, A. S. (2017). Co-design, organizational creativity and quality improvement in the healthcare sector:'Designerly’or ‘design-like’. In D. Sangiorgi \& A. Prendville (Eds.), Designing for service: Key issues new directions (pp. 117-128). London: Bloomsbury academic.

Robert, G., Cornwell, J., Locock, L., Purushotham, A., Sturmey, G., \& Gager, M. (2015). Patients and staff as codesigners of healthcare services. BMJ (Online), 350. https://doi.org/10.1136/bmj.g7714

Sanders, E. B. N., \& Stappers, P. J. (2008). Co-creation and the new landscapes of design. CoDesign, 4(1), 5-18. https:// doi.org/10.1080/15710880701875068

Sanders, E. B. N., \& Stappers, P. J. (2014). Probes, toolkits and prototypes: three approaches to making in codesigning. CoDesign, 10(1), 5-14. https://doi.org/10.1080/15710882.2014.888183

Seekins, T., \& White, G. W. (2013). Participatory action research designs in applied disability and rehabilitation science: protecting against threats to social validity. Arch Phys Med Rehab, 94(1), S20-S29. https:/doi. org/10.1016/j.apmr.2012.07.033

Sheeran, P., Klein, W. M., \& Rothman, A. (2017). Health behavior change: Moving from observation to intervention. Ann Rev Psychol, 68, 573-600. https://doi.org/10.1146/annurev-psych-010416-044007

Stappers, P., \& Giaccardi, E. (2017). Research through design. In The Interaction Design Foundation. (Ed.), The encyclopedia of human-computer interaction (2 ${ }^{\text {nd }}$ edition). https://www.interaction-design.org/literature/book/ the-encyclopedia-of-human-computer-interaction-2nd-ed/research-through-design\#chapter_start

Steen, M. G. D. (2008). The fragility of human-centred design (Doctoral Dissertation) Delft University of technology. http://resolver.tudelft.nl/uuid:2b930c09-0ad3-41f8-93f0-503eaea04ff0

Tarquinio, C., Kivits, J., Minary, L., Coste, J., \& Alla, F. (2015). Evaluating complex interventions: Perspectives and issues for health behaviour change interventions. Psychol Health, 30(1), 35-51. https://doi.org/10.1080/08870 446.2014.953530

Toye, F., Seers, K., Hannink, E., \& Barker, K. (2017). A mega-ethnography of eleven qualitative evidence syntheses exploring the experience of living with chronic non-malignant pain. BMC Med Res Methodol, 17(1), 116. https:// doi.org/10.1186/s12874-017-0392-7

Trebble, T. M., Hansi, N., Hydes, T., Smith, M. A., \& Baker, M. (2010). Process mapping the patient journey: An introduction. BMJ, 341, c4078. https://doi.org/10.1136/bmj.c4078

Turk, D. C., \& Rudy, T. E. (1991). Neglected topics in the treatment of chronic pain patients-relapse, noncompliance, and adherence enhancement. Pain, 44(1), 5-28. https://doi.org/10.1016/0304-3959(91)90142-k 
Verbiest, M. E., Corrigan, C., Dalhousie, S., Firestone, R., Funaki, T., Goodwin, D., Grey, J., Henry, A., Humphrey, G., \& Jull, A. (2018). Using codesign to develop a culturally tailored, behavior change mHealth intervention for indigenous and other priority communities: A case study in New Zealand. Transl Behav Med. https://doi. org/10.1093/tbm/iby093

Visser, F. S., Stappers, P. J., Van der Lugt, R., \& Sanders, E. B. (2005). Contextmapping: experiences from practice. CoDesign, 1(2), 119-149. https://doi.org/10.1080/15710880500135987

Verbiest, M. E. A., Corrigan, C., Dalhousie, S., Firestone, R., Funaki, T., Goodwin, D., Grey, J., Henry, A., Humphrey, G., Jull, A., Vano, M., Pekepo, C., Morenga, L. te, Whittaker, R., \& Mhurchu, C. N. (2019). Using codesign to develop a culturally tailored, behavior change mHealth intervention for indigenous and other priority communities: A case study in New Zealand. Trans Beh Med, 9(4), 720-736. https://doi.org/10.1093/tbm/iby093

White, G. W., Suchowierska, M., \& Campbell, M. (2004). Developing and systematically implementing participatory action research. Arch Phys Med Rehab, 85, S3-12. https://doi.org/10.1016/j.apmr.2003.08.109

Yin, R. K. (2003). Case study research design and methods, third edition. Thousand Oaks: SAGE publications. 


\section{Phase 1 "discover"}

\subsection{Kick off}

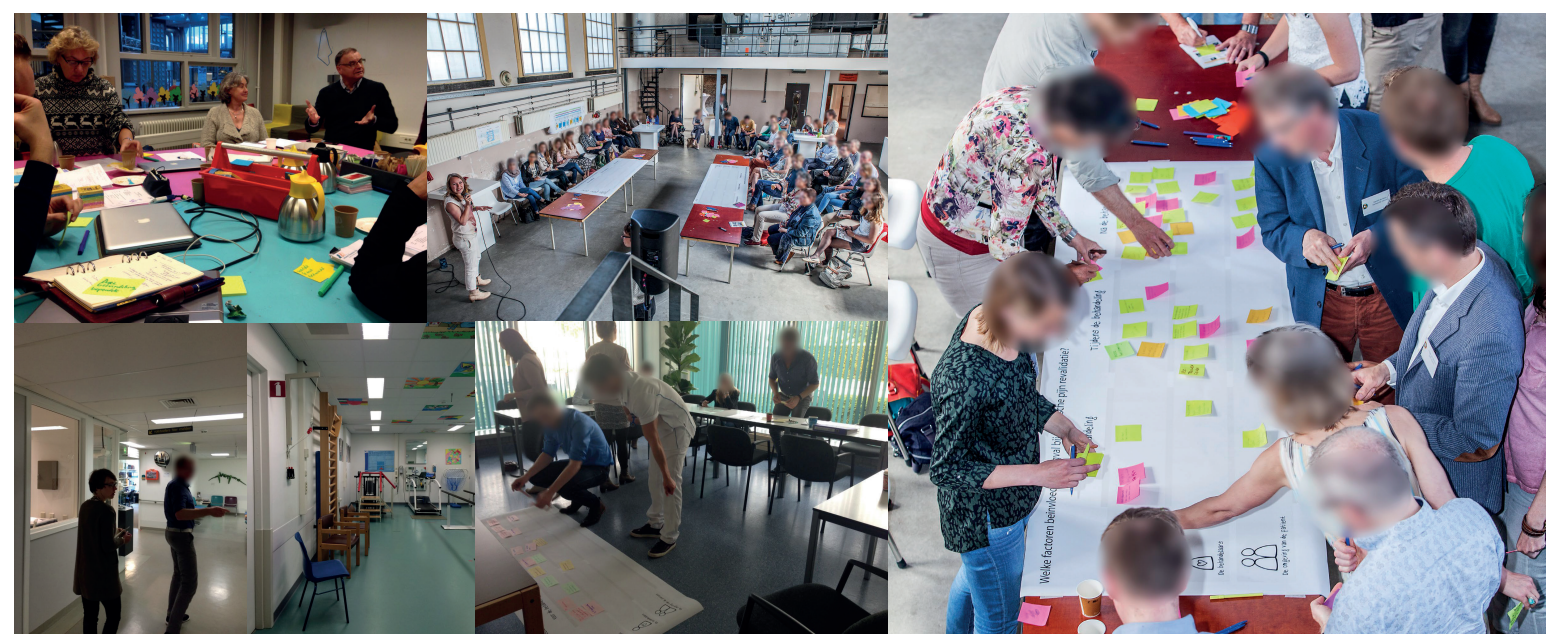

An illustration of the kick-off sessions, including location visits, the first consortium meeting, and the formulation of stakeholders' initial ideas on relapse. For this purpose, we used a patient journey, that not only facilitated the generation of insights, but also transformed the room into a design space.

Empty Journey map Example of a generative technique that facilitated participants to share their initial ideas and experiences that were related to relapse. Time (before, during and after treatment) is depicted on the $x$-axis, and important stakeholders (i.e. patient, healthcare providers and their immediate social system) are depicted on the $y$-axis.
Which factors may influence relapse after chronic pain treatment?

Prior to treatment

Durins treatment

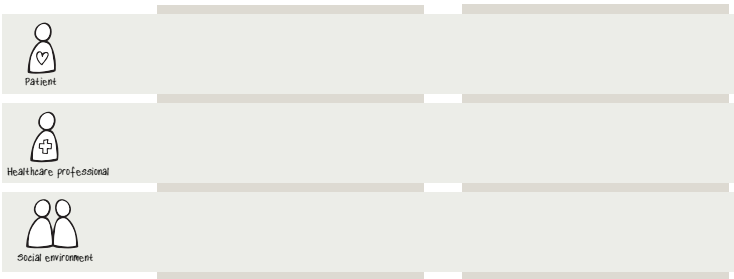

Post-treatment

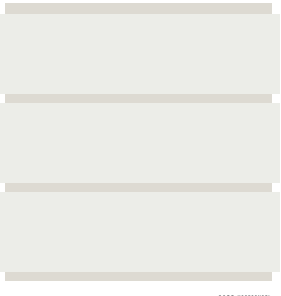




\section{Phase 1 "discover" \\ 1.2 Interviews}

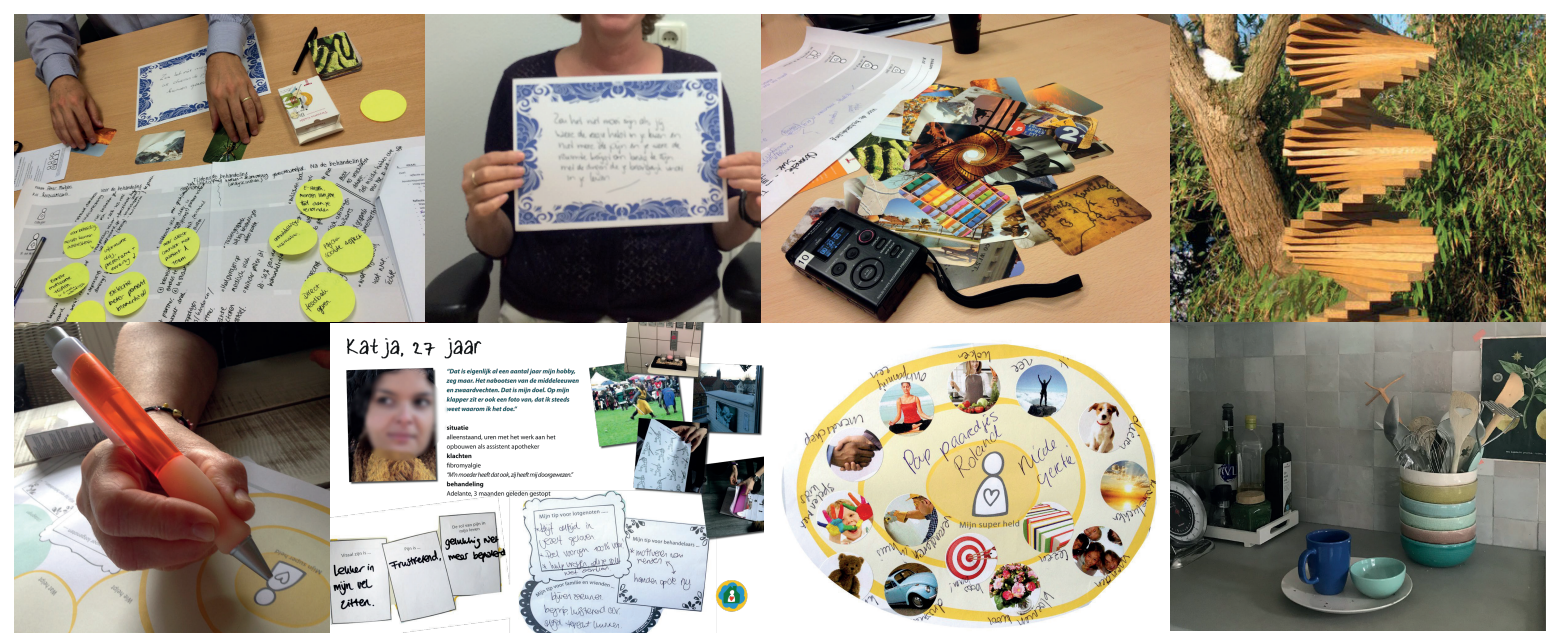

Illustration of stakeholder interviews, including the 'ambiguous images' that were used during the introduction, journey maps and a an assignment to write down a personal desire ("wouldn't it be nice if ...") related to improving the care for patients with chronic pain. Patient were interviewed in their personal context.

Generative techniques for patient interviews

Prior to the interview, a patient received a 'sensitizer' (left) to pre-activate thoughts and ideas related to their experience (e.g. description of a day were they felt particularly well or restricted by the pain). During the interview both the patient and interviewer collaborated to construct a patient journey map that laid on the table (right). The stickers aimed to elicit associations or to bring up tacit knowledge.

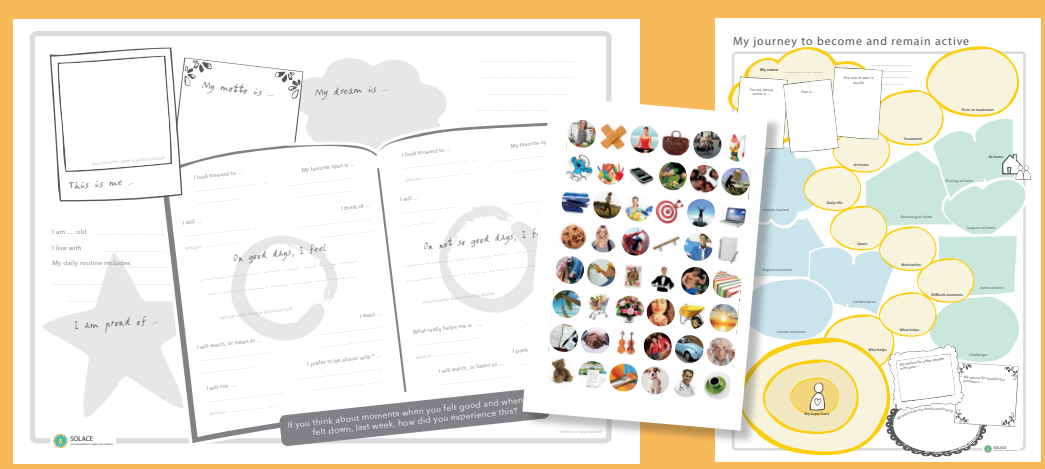




\section{Phase 1 "discover" \\ 1.3 Student design projects}

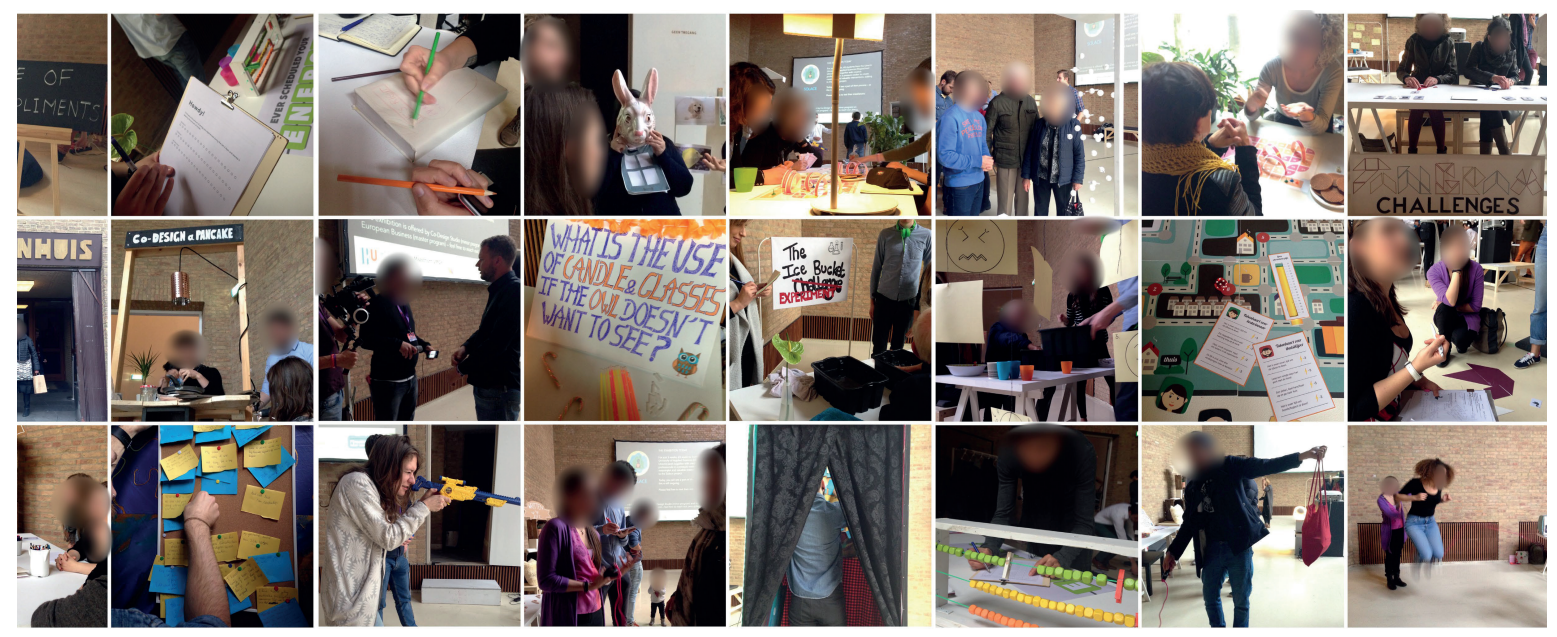

Example of various student teams that tested specific hypotheses related to relapse. Participants (e.g. bystanders or patients) interacted with provotypes to acquire insights.

\section{Provotypes}

Two examples of provotypes that were developed and used by the student teams. 'Spirit' was a board game were patients and their families had to cooperate to successfully complete a normal day, including work, school and all required tasks. Importantly, participants had to switch roles and play a different family member. This tested the idea that sharing details of coping with pain in daily life with family would reinforce empathy and mutual understanding. In 'Co-design a pancake', patients and significant others had to collaborate to bake a pancake, which was further stimulated by modifying the kitchenware (e.g. a ladle with two handles). This tested the assumption that pain could interfere with equality as partners.
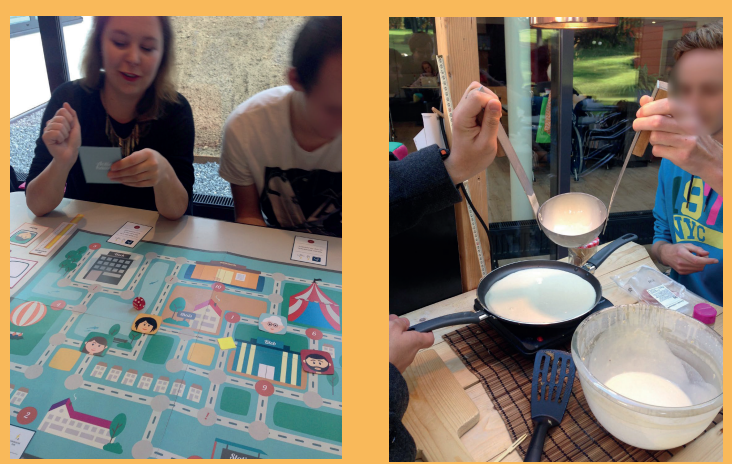


\section{Phase 2"define" \\ 2.1 Analyzing session}

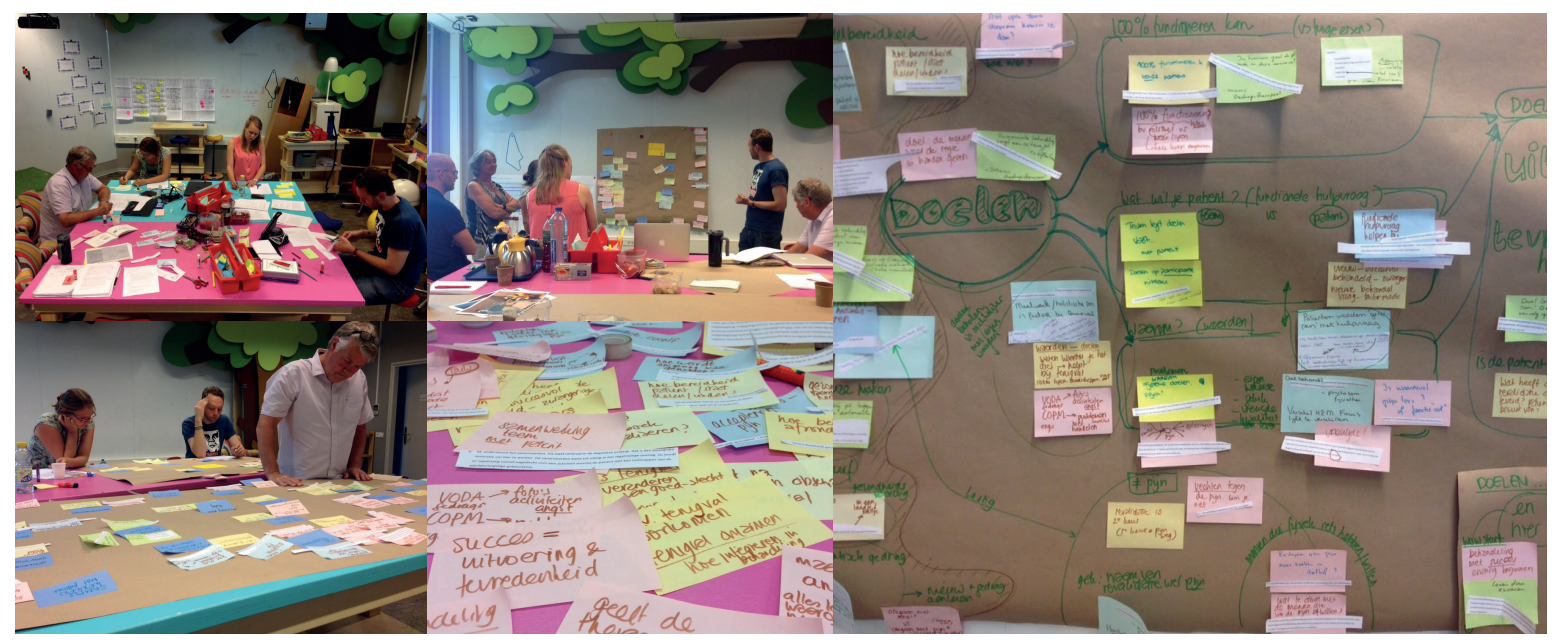

Overview of analysing sessions with the core teams to organize and cluster the interview and kick-off data. Relevant data segments were placed on post-its and organized around emerging insights and clustered in overarching themes, in shared within the team.

System map

The system map for the first co-creation session consisted of 8 posters that each contained a theme (rephrased as a question), subthemes and corresponding quotes and pictures. In addition, 74 stimulus cards were developed that each contained a specific insight or principle.
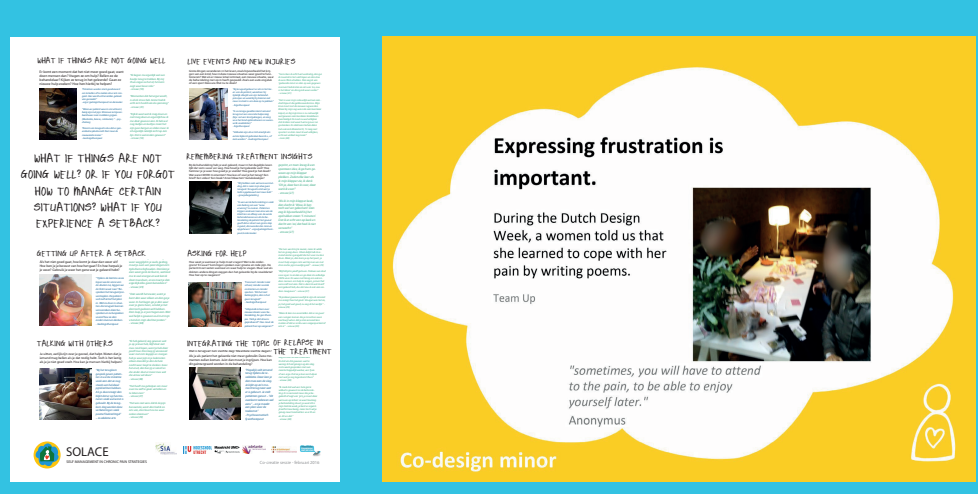

\section{SOLACE}




\section{Phase 2'define' \\ 2.2 Posters}

Overview of themes, subthemes and exemplary quotes of the data that was collected in the 'discover' phase.

\begin{tabular}{|c|c|c|}
\hline Themes & Subthemes & Quotes \\
\hline $\begin{array}{l}\text { 1. Emptiness after treatment } \\
\text { How will you cope with being on your own, } \\
\text { after treatment? How do you keep believing } \\
\text { in yourself and remain focused on important } \\
\text { treatment insights? }\end{array}$ & $\begin{array}{ll}\text { 1. } & \text { Emptiness } \\
\text { 2. Pride } \\
\text { 3. Responsibility } \\
\text { 4. Effective relationship with HCPs } \\
\text { 5. Confidence } \\
\text { 6. How to remain motivated to remain } \\
\text { focused on treatment insights by } \\
\text { yourself } \\
\text { 7. Post-treatment contact between patient } \\
\text { and HCP }\end{array}$ & $\begin{array}{l}\text { "I am proud of my children and grandchildren. And I am also proud of what I achieved during the } \\
\text { treatment program." } \\
\text { - patient, } 1.2 \\
\text { "Confidence is an important topic during treatment. It helps if you show that you are confident that a } \\
\text { patient is able to achieve his or her goals." } \\
-H C P, 1.5\end{array}$ \\
\hline $\begin{array}{l}\text { 2. Goals and values } \\
\text { How do you maintain focus on your goals and } \\
\text { values? How do you remain engaged? How do } \\
\text { you stay on track rather than being distracted by } \\
\text { the pain? }\end{array}$ & $\begin{array}{l}\text { 1. Remembering goals and values } \\
\text { 2. Differences between treatment centre } \\
\text { 3. } \quad \text { and personal environment } \\
\text { 4. Planning daily activities } \\
\text { 5. Motivation } \\
\text { 6. }\end{array}$ & $\begin{array}{l}\text { "Walking was my most important goal. Why? Because it gives me freedom." } \\
\text {-patient, } 2.1 \\
\text { "You will not win the battle against the pain.. wouldn't it be nice if to focus shifted from pain to general } \\
\text { enjoyment of life?" } \\
-H C P, 2.6\end{array}$ \\
\hline
\end{tabular}

3. Coping with a setback

What if things are not going well? Or if you forgot how to manage certain situations? What if you experience a setback?

\section{What if things are not going well}

2. Live events and new injuries Remembering treatment insights Getting up after a setback

Asking for help

Talking with others

Talking with others treatment
"She [therapist] taught me to use the [graded activity] schedule for a couple of days, in case that I fall back into old habits." -patient, 3.4

"I think it is a good thing if patients experience a setback during treatment. Than you can use this experience to make prevention plans in anticipation of future events."

$-H C P, 3.7$
4. Skills to prevent relapse

What are important skills to prevent relapse? How can you train or promote these skills? How do they become a part of you?
1. Creativity

2. Impulsivity and courage

Positivity, joy and humo

Resilience, acceptance, letting-go

Reflecting

Self-compabssion

Distraction, shifting focus
Creativity is an important trait. If a strategy does not work, patients will have to find new solutions - $H C P, 4.1$

"I learned to have self-compassion. Before treatment, I used to punish myself, but now, I don't do that anymore. If you like yourself, you are also prepared to take good care for yourself." - patient, 4.6
5. Involving significant others

How do you explain your treatment experiences to your significant others? How do you

communicate that you have changed and what your new goals are?

Sharing experiences
Support and understanding
Barriers within the family
Habits
Attention

5. Attention
"A patient can change, but if the social environment is not sufficiently supportive the chance of relapse will increase. That's why it is important to involve the family during treatment." - HCP 5.2

"My husband always wanted to care for me and to ensure that nothing bad happened. That was difficult for me, I had to ask him to stop interfering."

-patient, 5.3

\section{Personal boundaries, pacing and} monitoring energy

How do you remain true to yourself and

communicate your boundaries? How do you

manage your energy levels and remain relaxed?
1. Rest

2. Making decisions and setting personal boundaries

3. High demands

4. Saying 'no'

5. Energy management

6. Limited by pain

"The treatment is successful if a patient regained control over his life. Not the pain is central, but his aims and desires in life."

$-H C P, 6.4$

"Planning my daily schedule in advance was useful for me. I learned how to distribute my time and energy by adhering to the schedule."

Patient 6.5

\section{Improving after treatmen}

How can you continue to improve after

treatment?

End of treatment
Treatment successes?
Setting new goals
Back to the old personal environment

. End of treatment

4. Back to the old personal environment
"We used to celebrate the end of a treatment period with a party and a couple of beers. But we don't do that anymore. We do have a conversation about what we learned and how they experienced the program." - group coach, 7.1

"After fifteen years of pain, I immediately wanted to check if I was ok, after treatment. I started kickboxing, which actually went quite well." - patient, 7.3

\section{Insight into own behaviours}

How do you acquire insight into your own

behaviour and how things are going? How

do you monitor your current level and your

progress? How do you organize feedback and

how should you ideally respond to this?
"I learned to have more confidence in my body. That you actually can do whatever you want, but sometimes you need to do it slightly different than the rest.".

- patient, 8.

"Innovations and eHealth are opportunities to monitor patients after treatment and to signal relapse. That could be combined with online sessions with a psychologist."

HCP, 8.2 
Example of a poster that was created for each of the themes (headings translated).

WHAT IF THINGS ARE NOT GOING WELL

Er komt een moment dat het niet meer goed gaat, want doen mensen dan? Wragenze om hulp? Bellenze de nieuwe hulp zoeken? Hoe hen hierbij te helpen?
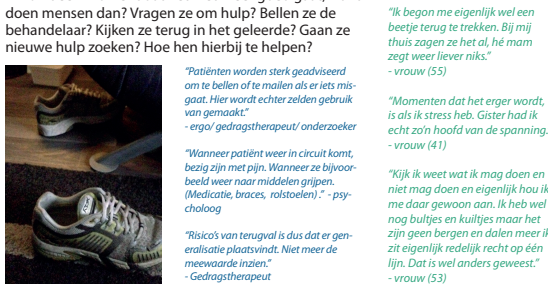

WHAT IF THINGS ARE NOT GOING WELL? OR IF YOU FORGOT HOW TO MANAGE CERTAIN SITUATIONS? WHAT IF YOU EXPERIENCE A SETBACK?

GETTING UP AFTER A SETBACK

Als het niet goed gaat, hoe komt je daar dan weer uit? Hoe benjejebewst vanhoehet gath Enhoe herpakj
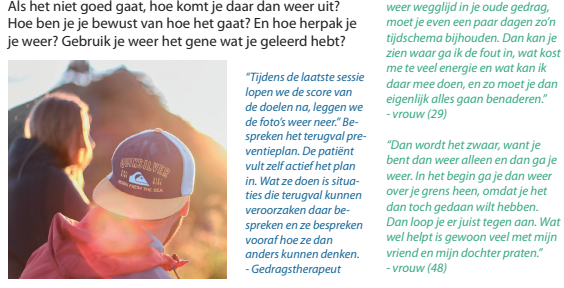

TALKING WITH OTHERS

Je uitten, eerlijk zijn over je gevoel, dat helpt. Weten dat je iemand mag bellen als je dat nodig hebt. Toch is het lastig
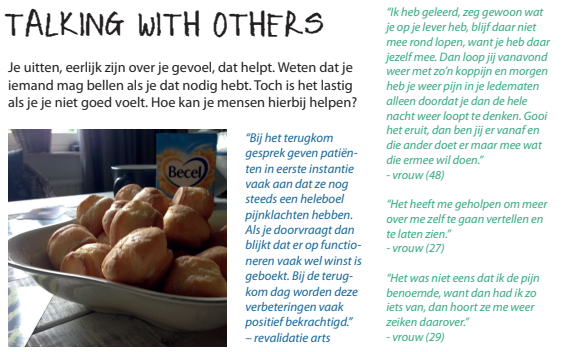

LIVE EVENTS AND NEW INJURIES
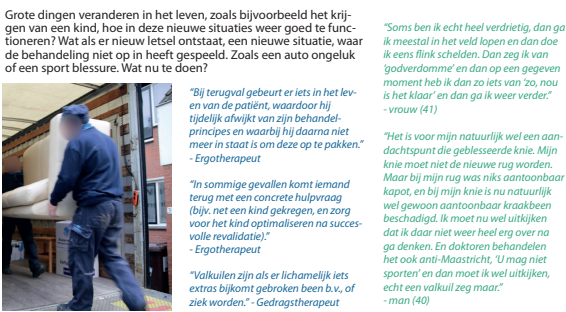

\section{REMEMBERING TREATMENT INSIGHTS}
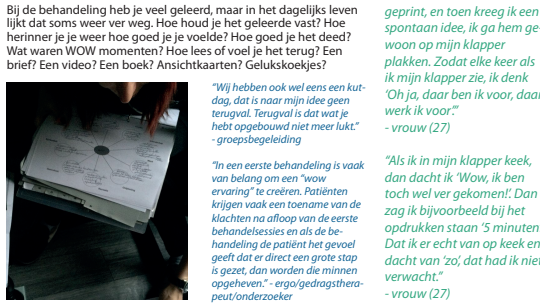

ASKING FOR HELP
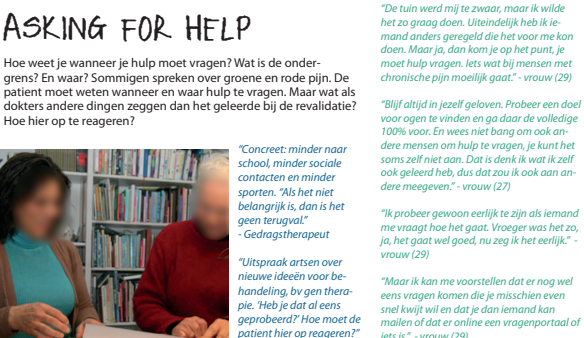

INTEGRATING THE TOPIC OF RELAPSE IN Watis terugval? Een slechte dag? Meerdere slechte dagen? THE TREATMENT Als je als patient het geleerde niet meer gebruikt. Dezemodit geintergreerd worden in de behandeling?
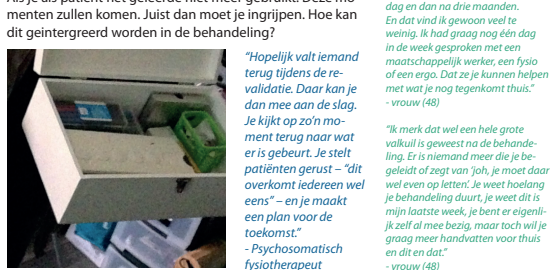

\section{SOLACE}




\section{Phase 2 'define'}

\subsection{Cardset}

Examples of four stimulus cards that where

developed for the first co-creation session.
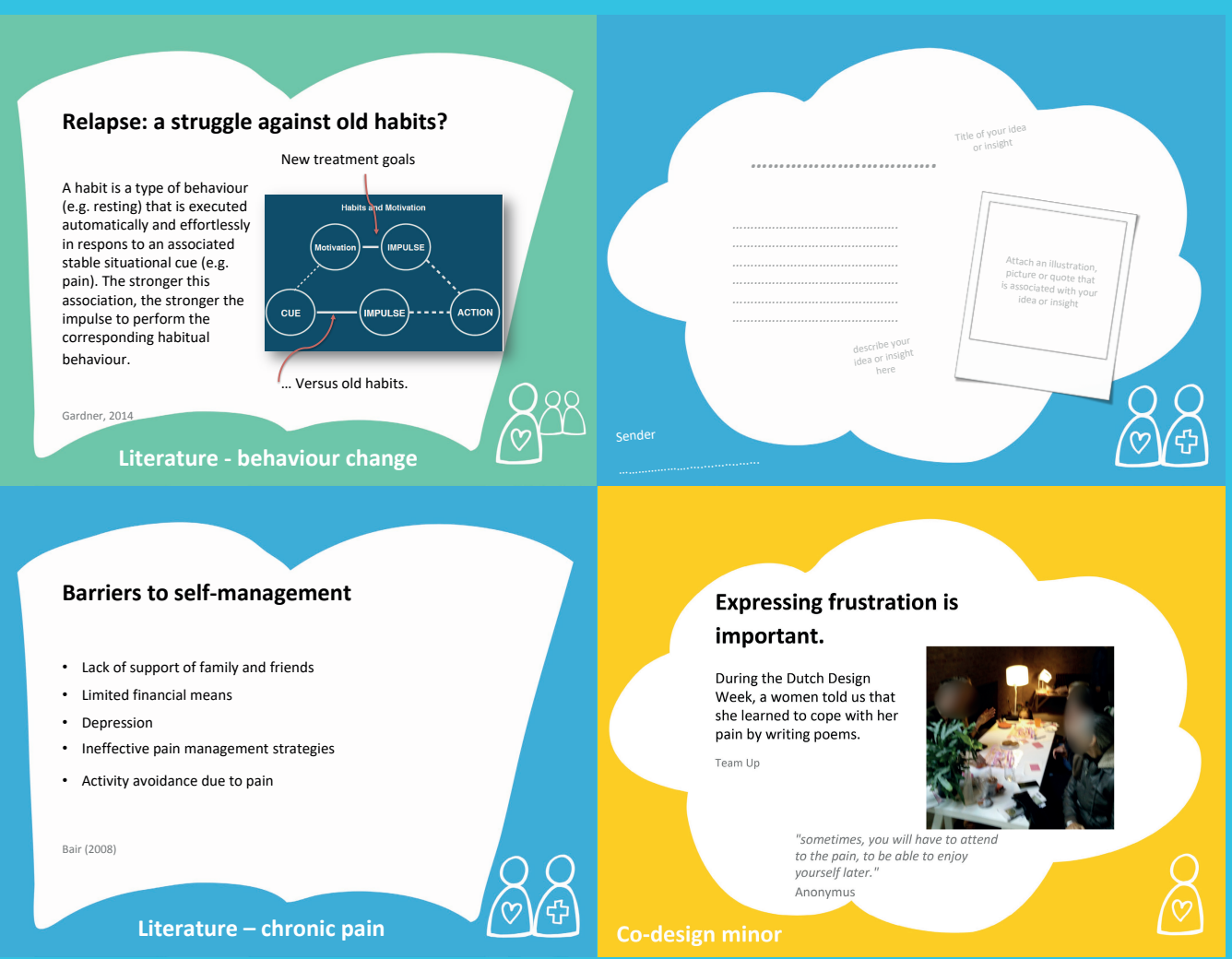

- Lack of support of family and friends

- Limited financial means

- Depression

- Ineffective pain management strategies

- Activity avoidance due to pain

Bair (2008)

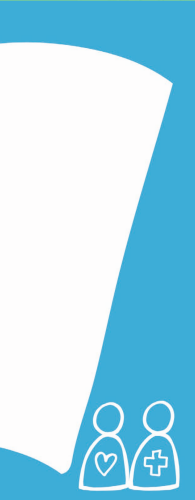




\section{Phase 2"define"}

\subsection{Co-creation session 1}

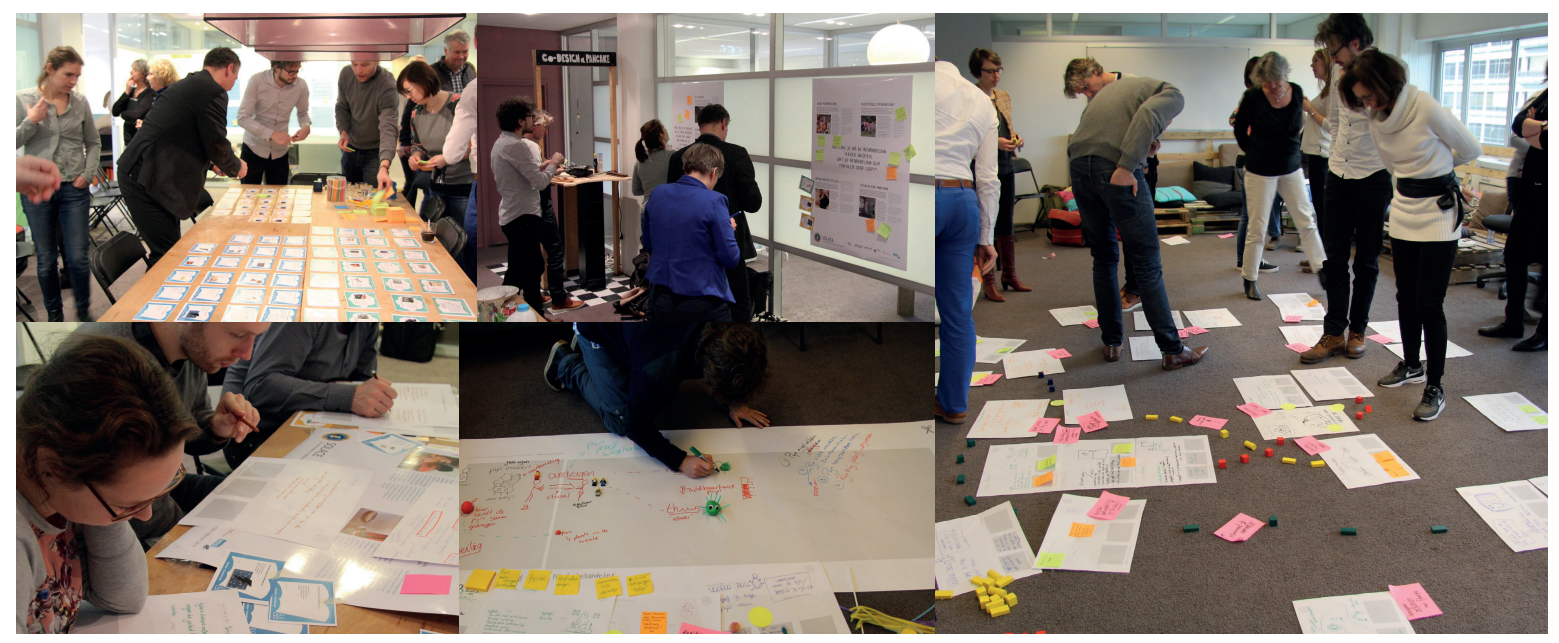

Overview of the co-creation session. By means of various assignments, stakeholders were encouraged to interact with each other and to use the data to develop intervention concepts.

Five concept ideas Throughout the session, a professional draftsman visualized the emerging concept ideas. For example, 'time machine' included making videos to register progress and to allow 'time travel' trough earlier treatment experiences.

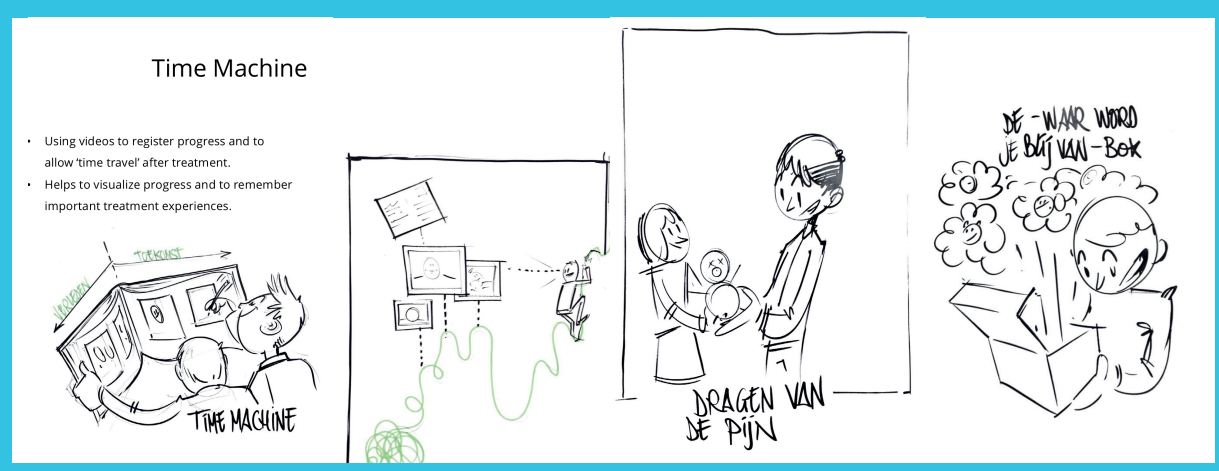




\section{Phase 3 "develop"}

\subsection{Concept development}

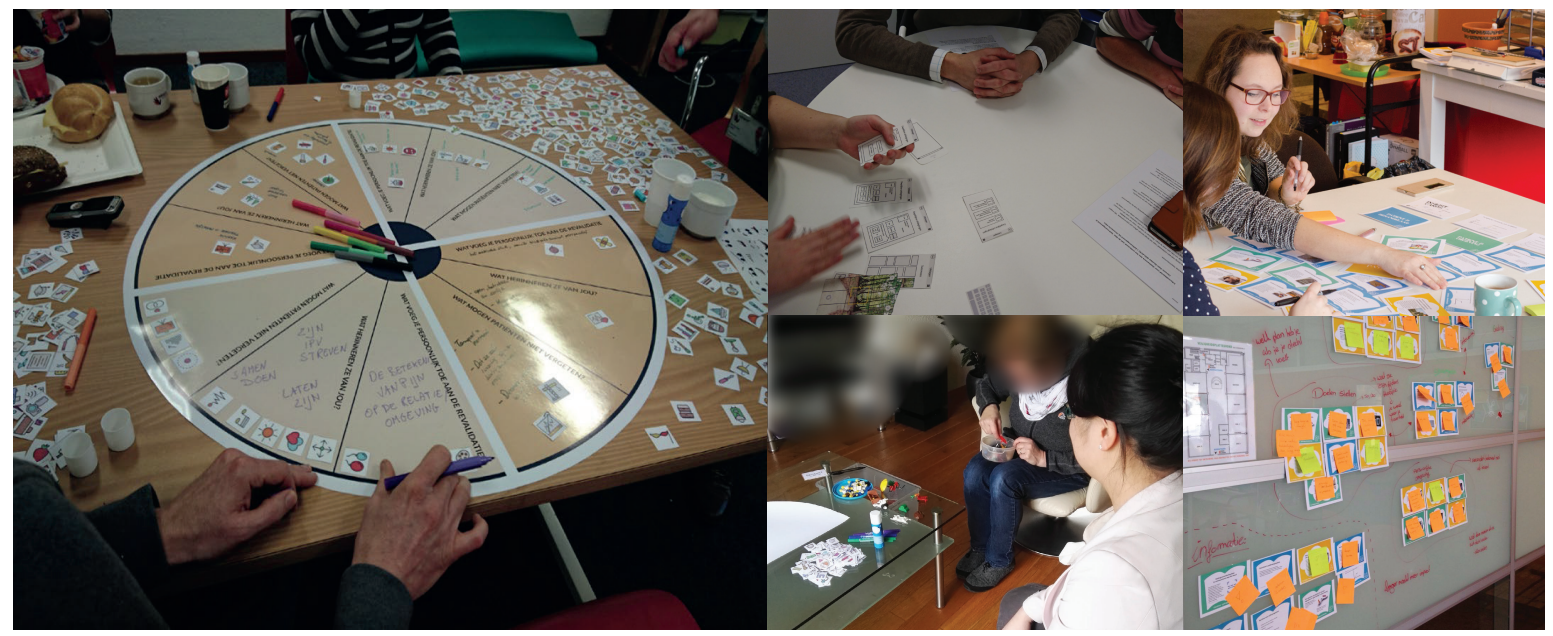

Pictures that were taken during various stakeholder and analysing sessions in the develop phase. Two student teams further developed the concepts into rudimentary prototypes. They regularly involved patients and health providers to test and refine their ideas.

Two rudimentary prototypes The end result of the develop session two rudimentary prototypes.
SOLAPP MOBIELE APPLICATIE

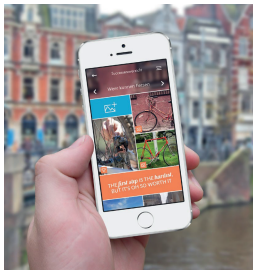




\section{Phase 3 'develop'}

\subsection{DIY Toolkit}

The DIY toolkit contains various means to capture and store relevant treatment insights. These means are grouped by the corresponding type of storage: visually (e.g. a picture); by text (e.g. an associated quote); or by sound (e.g. a voice recording). The toolkit also provides suggestions for how to store the insights after treatment and how to share this information with significant others or healthcare providers.

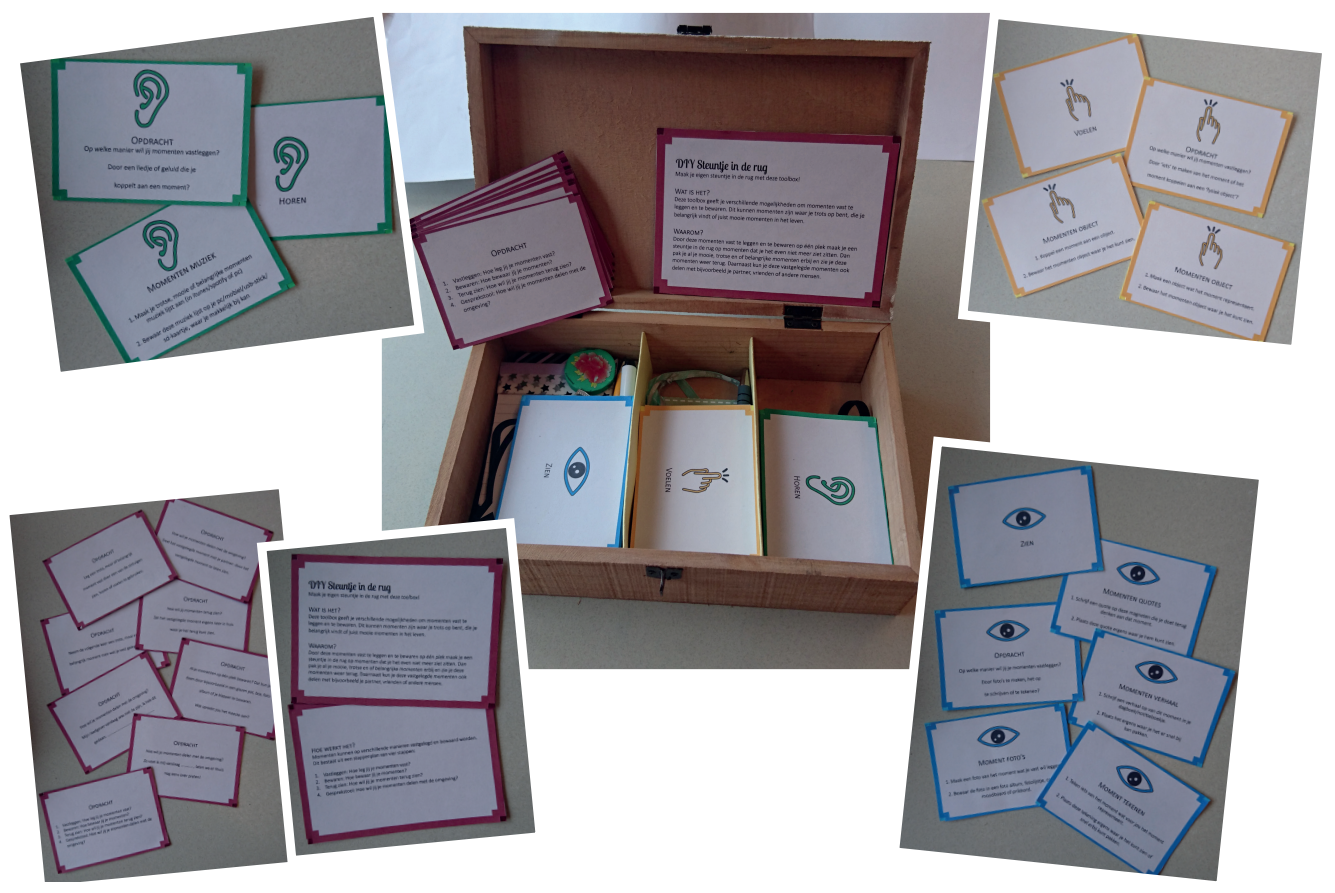




\section{Phase 3 'develop' \\ 3.3 Solapp}

The Solapp application provides patients with the opportunity to describe important personal values and subsequently formulate related personal goals. The application provides a structure where patients can gradually progress towards each goal by means of planning specific 'steps'.

\section{$S O L A P P$ \\ MOBIELE APPLICATIE}

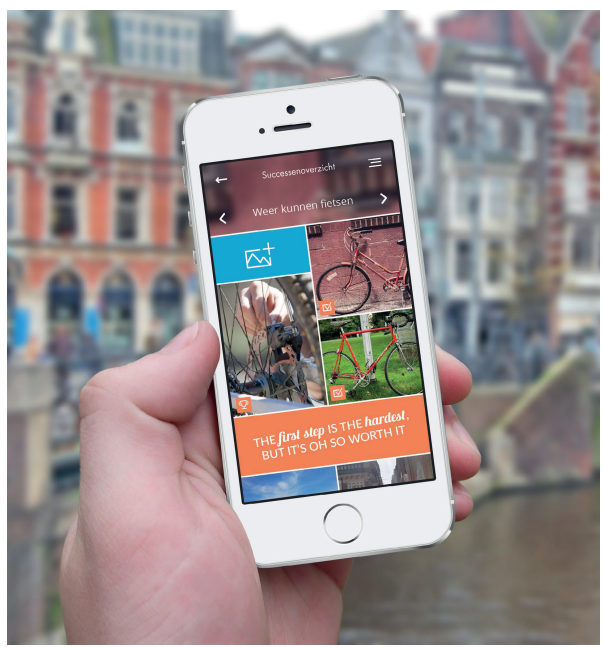

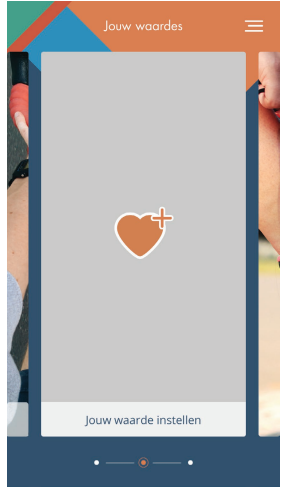

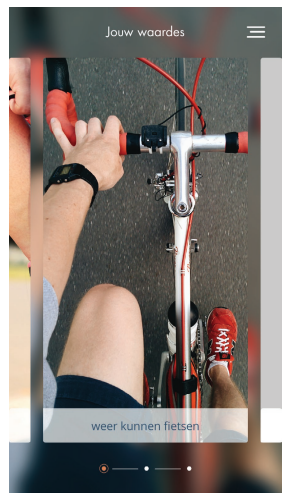

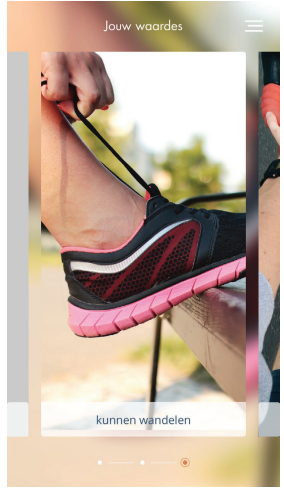

\section{OVERZICHT}

\section{WAARDES}

- Inzicht 7 - Visueel maken

- Inzicht 16

- Uitspraak 47

- Iteratie pag. 90

- Ontwerpcriteria 2

- Ontwerpcriteria 4

- Ontwerpcriteria 5

- Ontwerpcriteria 11

- Inzichtkaart 06

- Inzichtkaart 07

- Inzichtkaart 09

\section{SOLACE}




\section{Phase 4 "deliver"}

\subsection{Co-creation session 2}

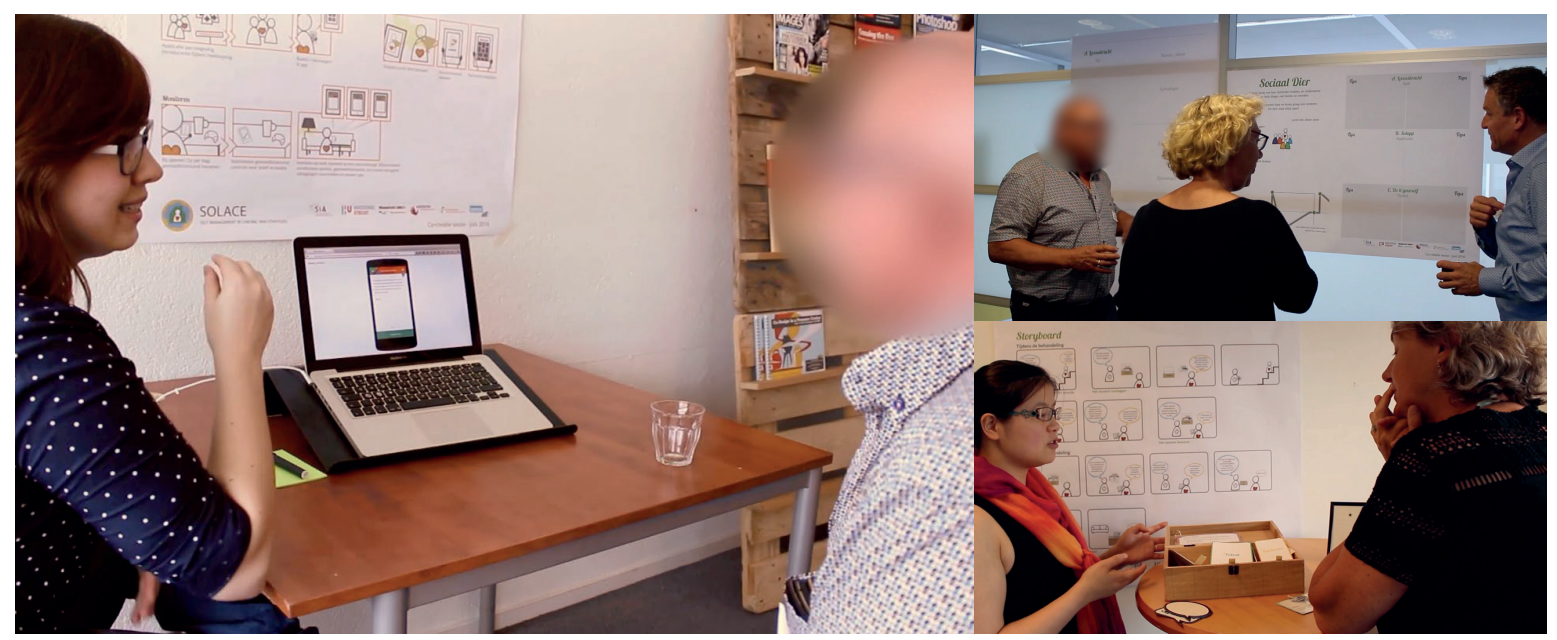

Overview of the co-creation session where students presented their prototypes and stakeholders discussed their use and function.

Personas

4 personas that were based on extremes two characteristics: extravert versus introvert and underuse versus overuse. This tool helped to simulate the adaptation of both prototypes for each of these four personas, providing an emphasis on the patient perspective.

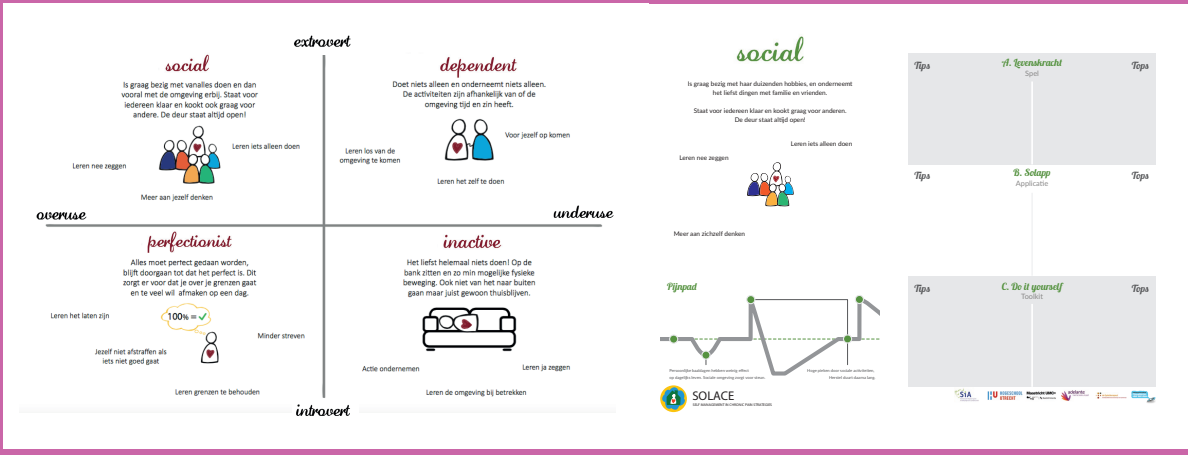




\section{Phase 4 'deliver'}

\subsection{Intervention components}

Both interventions were divided into multiple separate components. For each component we evaluated to what extent it corresponded with (sub)themes from the qualitative dataset. Furthermore, we performed literature searches to find ways to improve each component.

\section{For example:}

Component 'select personal values'

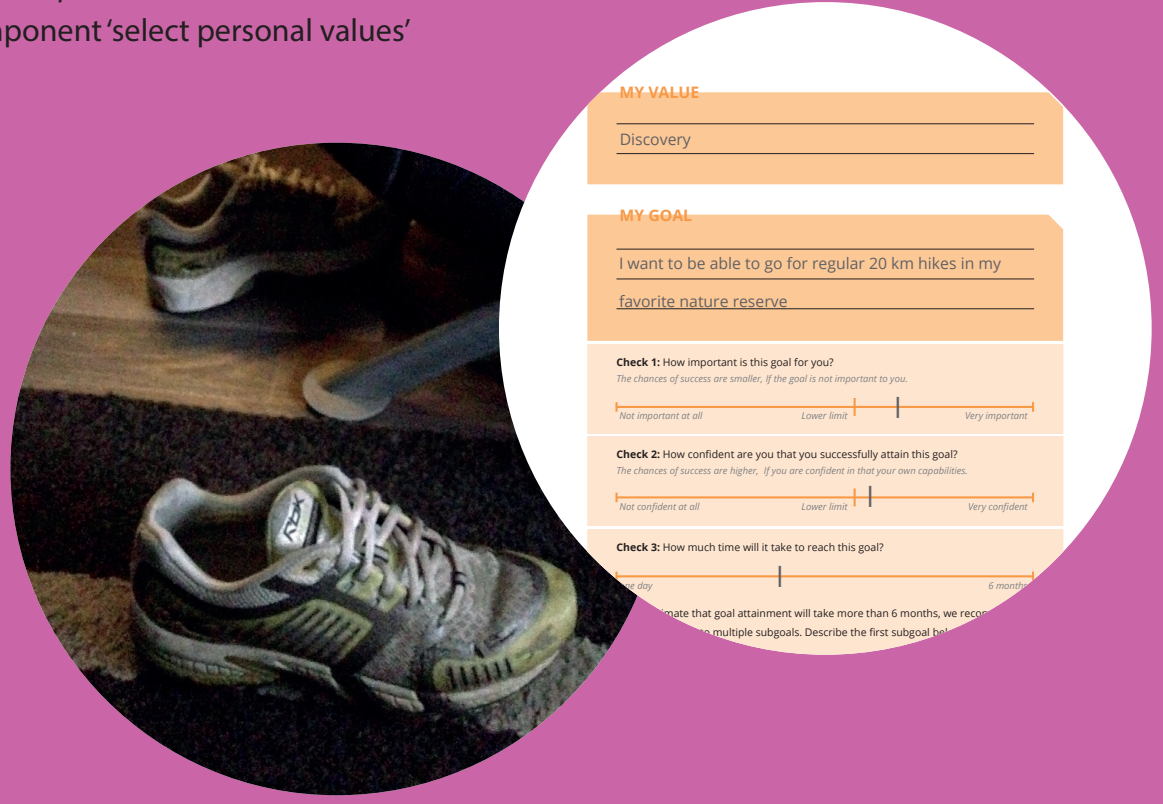

Theme 2_Goals and values

Subtheme 1_Remembering goals and values

"Walking was my most important goal. Why? Because it gives me freedom."

- patient, 2.1

\section{BCT 13.4_Valued self-identity}

Chase et al (2013) Values are not just goals

\section{SOLACE}

SELF MANAGEMENT IN CHRONIC PAIN STRATEGIES 


\section{Phase 4 'deliver'}

\subsection{Prototype workbook}

English translation of four pages of the workbook prototype:

a_front page

b/c_value based goalsetting procedure

d_example of two insight cards.

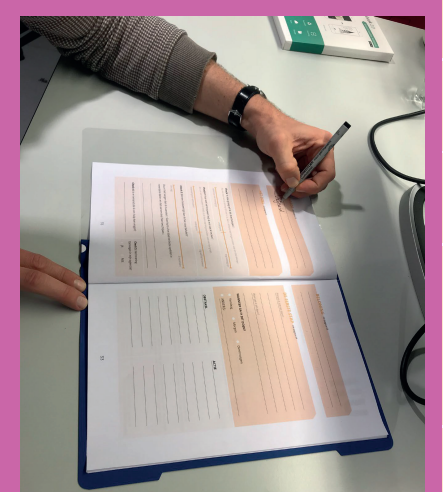

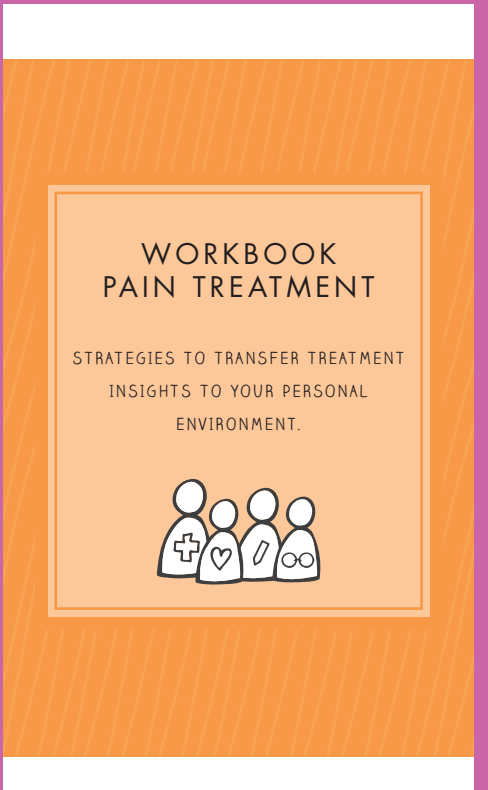

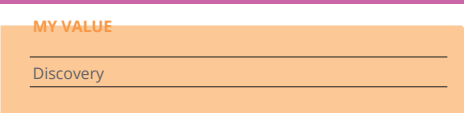

favorite nature reserve

mportant is this goal for yo

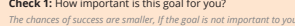

Not important of all

check 2. How confident are you that you successfully attain this goal?

Check 2: How confident are you that you successfully attain this goal?

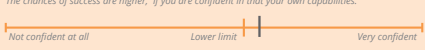

Check 3. How much time will it take to reach this goal

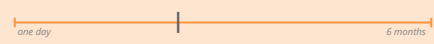

If you estimate that goal artainment will take more than 6 months, we recommend to divide the goal into multiple subgoals. Describe the first subroal below
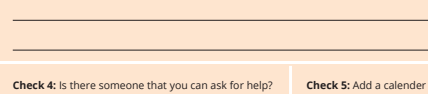

my friend Jane

Check 5: Add a calender

$\mathbf{X}$ YES NO

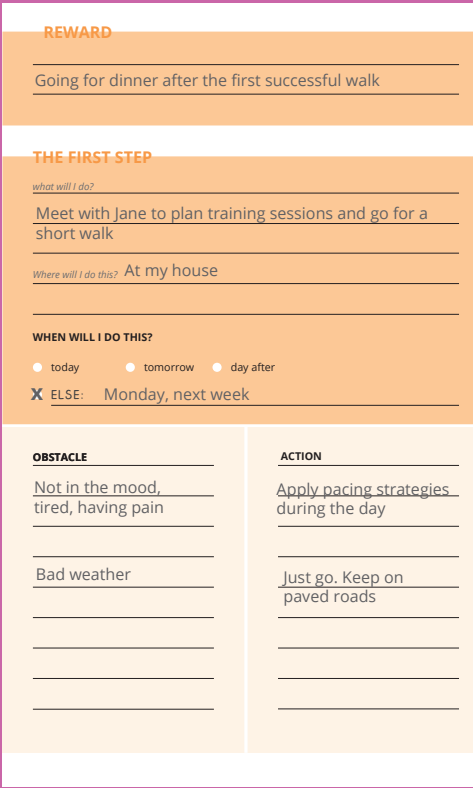

MOMENT

1 HAD A PLEASANT INTERACTION WITH A NEW

ENCOURAGED HIM TO CONTINUE EXERCISING. IRRESPECTIVE OF PAIN OR TIREDNESS.

MEMORY CUE

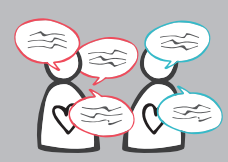

MOMENT IMPORTANT TOPIC DURING TODAY'S SESSION HURT DOES NOT EQUAL HARM.

MEMORY CUE

HURT $\neq$ HARM 



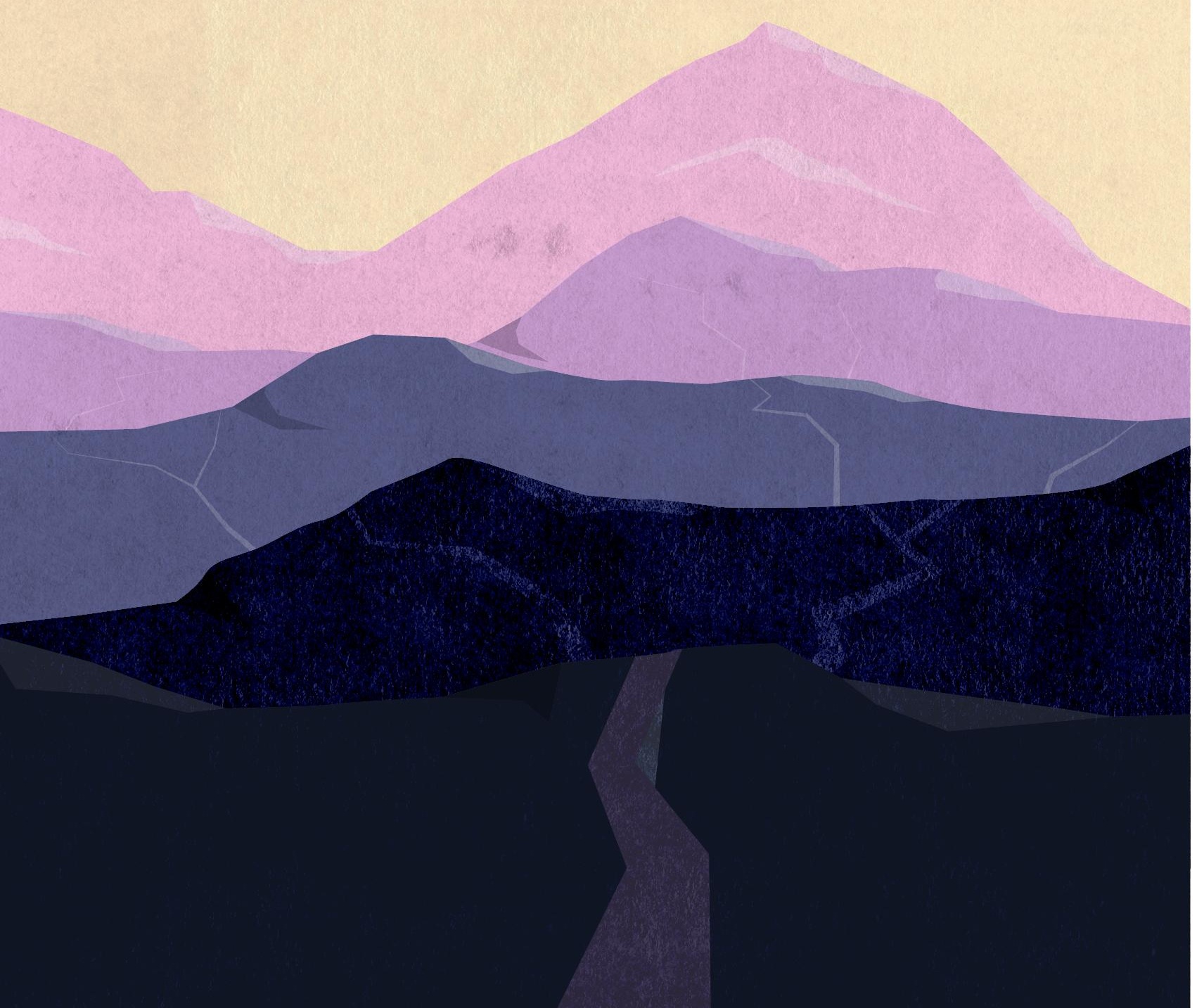




\section{6}

\section{Exploring the Feasibility of Relapse Prevention Strategies in Interdisciplinary Multimodal Pain Therapy Programs: Qualitative Study}

S. Elbers ${ }^{1,2}$, J. Pool ${ }^{1}$, H. Wittink ${ }^{1}$, A. Köke ${ }^{2,3,4}$, R. Smeets ${ }^{2,5}$

JMIR Human Factors (2020), 7(4), e21545

1. Research Group Lifestyle \& Health, Research Centre Healthy and Sustainable Living, University of Applied Sciences Utrecht, Utrecht, The Netherlands.

2. Department of Rehabilitation Medicine, Faculty of Health, Life Sciences and Medicine, Maastricht University, Maastricht, The Netherlands.

3. Centre of Expertise in Pain and Rehabilitation, Adelante, Hoensbroek, The Netherlands.

4. South University of Applied Sciences, Heerlen, The Netherlands.

5. Centrum voor Integrale Revalidatie, Eindhoven, The Netherlands. 


\section{ABSTRACT}

Background: Although interdisciplinary multimodal pain treatment (IMPT) programs are widely regarded as treatment of choice for patients with chronic pain, there are signs that many patients are unable to maintain their treatment gains in the long term. To facilitate the maintenance of positive treatment outcomes over time, we developed two relapse prevention strategies.

Objective: The main objective of this study was to explore the feasibility of these strategies within the context of IMPT programs.

Methods: We performed a feasibility study using 3 workbook prototypes containing either one or both strategies. For a period of 6 months, the workbooks were made available in two IMPT facilities. Qualitative data were collected through a focus group and semistructured interviews. We performed a thematic analysis using a deductive approach with (1) applicability to the treatment program, (2) acceptability of the workbook content, and (3) form, as predefined themes.

Results: The final dataset consisted of transcripts from a focus group with health care providers and 11 telephone interviews and 2 additional in-depth interviews with patients. In general, the intervention was perceived as useful, easy to use, and in line with the treatment program. The data also include suggestions to further improve the use of both strategies, including more specific implementation guidelines, revised goal-setting procedure, and development of a mobile health version. However, several factors, including a high dropout rate and small sample size, impact the external validity of our findings.

Conclusions: This study should be regarded as a first step in the process of transforming the prototype workbook into an effective intervention for clinical practice. Although these initial results indicate a favorable evaluation of both behavior regulation strategies within the workbook, this study encountered multiple barriers regarding implementation and data collection that limit the generalizability of these results. Future research efforts should specifically address the fidelity of HCPs and patients and should include clear procedures regarding recruitment and use of both relapse prevention strategies during treatment.

Key words: chronic pain; interdisciplinary treatment; rehabilitation; relapse; behavior change; goal setting; pain; behavior; feasibility; strategy; treatment. 


\section{INTRODUCTION}

Interdisciplinary multimodal pain therapy (IMPT) programs have been developed to address the complex multifaceted nature of chronic pain. Instead of directly treating the pain itself, IMPT programs offer a comprehensive approach to target mutually interacting cognitive, behavioral, emotional, biological, and social factors to improve daily life functioning and quality of life, irrespective of pain (Adams \& Turk, 2015; Gatchel et al., 2014; Morley \& Williams, 2015). Typically, these programs include an interdisciplinary team of at least three professionals from varying backgrounds that coordinate their therapeutic activities throughout the program in line with patient-centered goals and biopsychosocial treatment principles. Also, IMPT programs are generally provided within a single facility, and patients are actively engaged with their rehabilitation by means of exercises and tasks (Gatchel et al., 2014; Kaiser et al., 2017). Although IMPT programs are often considered treatment of choice for patients with chronic pain (Kamper et al., 2014), there are signs that a considerable proportion of patients are not able to maintain positive treatment outcomes over time (Morley, 2008; Morley et al., 2013; Turk \& Rudy, 1991). This problem of relapse is not limited to IMPT; other behavioral treatments show similar trends for various patient groups, including patients following orthopedic rehabilitation (Reuter et al., 2009) and patients with chronic diseases (DiMatteo, 2004; Lee et al., 2013). These results indicate that the problem of relapse may transcend disease-specific treatment.

One strategy that has been recommended to improve long-term effectiveness is to adjust the treatment program to specific individual patient characteristics, needs, and preferences (Miettinen et al., 2019; Noar et al., 2007). This tailoring is specifically relevant in the domain of IMPT because these types of treatment programs seldom target one type of behavior but a complex and patient-specific cluster of health behaviors, each associated with patientspecific personal and contextual factors (Steiner, 2012). Moreover, in the context of IMPT, the options to realistically simulate a patient's natural environment within the boundaries of a treatment facility are limited, which may threaten effective generalization of newly learned behaviors to patient-specific meaningful contexts (Hollander et al., 2010).

To provide patients and health care providers (HCPs) with tools that could prevent relapse after successful treatment, we initiated a research project to develop a relapse prevention intervention. In iterations over a period of 18 months, patients, HCPs, pain researchers, experts on behavior change. and designers participated in co-design activities to develop two relapse prevention strategies. These activities ranged from interviews with patients and HCPs to full-day cocreation sessions where ideas were developed into concrete prototypes. In the final phase of the project, the two most promising relapse prevention strategies were merged into a paper prototype intervention. This relapse prevention workbook 
was regarded as the optimal form to test both strategies within existing IMPT programs, given the available budget and development time. The strategies within the workbook were based on experience from practice (e.g., patient interviews, stakeholder discussions, HCP feedback) and general self-regulation principles that relate to maintenance of newly learned behaviors (e.g., habit formation and goal setting) (de Ridder et al., 2008; Elbers et al., 2021; Kwasnicka et al., 2016; Nicholas et al., 2011). The main goal of the workbook was to support and facilitate the transfer of individually meaningful insights and learned skills to the personal context of each patient. Treatment teams provided feedback on the workbook design to ensure that the form and content would fit existing treatment principles (Wood \& Rünger, 2016).

To determine if these strategies are eligible for further development and efficacy testing, it is important to investigate their potential in a real-world setting. According to Bowen and colleagues (Elbers et al., 2021), feasibility studies are important to select promising interventions for further development and obtain specific feedback regarding factors such as usability and implementation.

To provide insight into patient and HCP evaluations regarding the relapse prevention strategies, our primary study objective was to examine the level of acceptability to stakeholders of the current workbook prototype within the context of IMPT programs. To explore how well the workbook fits within the existing treatment program, we additionally investigated the degree of applicability.

\section{METHODS}

\section{Study Design}

To investigate the feasibility of the strategies, we conducted a qualitative study to assess patient and HCP evaluations related to acceptability and applicability of the prototype intervention within the existing treatment program. Ethical approval was granted by the local ethics committee (Medical Research Ethics Committee Zuyderland 16-N-46).

\section{Participants}

The study was performed in two locations of the Adelante Rehabilitation Centre: Hoensbroek and Maastricht. Patients with chronic musculoskeletal pain were referred to the program by general practitioners or specialists who determined that primary care treatment was insufficient to address all existing biopsychosocial factors. Patients were eligible for treatment when their pain and pain-related disability interfered with daily life functioning to a moderate or severe extent. Patients could not participate in the 
rehabilitation program if they had severe or dominant psychiatric conditions, were unable to speak Dutch, were involved in ongoing legal procedures, or insisted on obtaining additional somatic diagnostic procedures. An additional criterion for location Maastricht was that patients needed to experience pain-related fear of performing certain activities in daily life that could be challenged in an in vivo exposure procedure. In this study, all patients who participated in the IMPT were eligible for inclusion.

\section{Treatment}

The outpatient IMPT programs at both locations varied in dose and content but had a similar biopsychosocial perspective and included pain neuroscience education as well as cognitive behavioral approaches to improve physical functioning and health-related quality of life. At minimum, the treatment staff consisted of a physiatrist, physical therapist, and psychologist but could also include a social worker and occupational therapist. Both programs required active patient involvement and included regular interdisciplinary team meetings to discuss the patient's progress. The treatment program in Hoensbroek contained multiple intervention components, including but not limited to graded activity, acceptance and commitment therapy, learning to pace (work-related activities) and set realistic goals under supervision of an occupational therapist, and functional exercise therapy such as swimming or walking under supervision of a physiotherapist. After an initial treatment phase of 3 weeks ( 5 days per week), a patient-specific program was created that matched dose and content with individual needs. On average, the total program was 12 weeks. The center provided accommodations for patients who were unable to commute to the center on a daily base. Location Maastricht primarily provided exposure in vivo treatment (Bowen et al., 2009; den Hollander et al., 2016; Hollander et al., 2010). At minimum, the program was 2 weeks with 2 treatment sessions per week but could be extended up to 10 weeks depending on the complexity of the case. A typical treatment program contained 20 hours of treatment and consisted of medical education by a physiatrist (that no harm or additional injury could be inflicted by performing activities) and behavioral experiments led by a physical or occupational therapist and psychologist (half of the sessions were led by both HCPs).

\section{Materials}

We developed two strategies to prevent relapse after successful treatment: Insight Cards and Value-Based Goal Setting (VBG). Insight Cards consisted of a set of cards on which patients could write down their most meaningful rehabilitation experiences, ideas, and milestones (Supplement 1). The upper half of the card provided space for the insights, and the bottom half was reserved for a related environmental cue such as a picture or a quote. The collection of these cards allowed HCPs to ensure the intervention was received 
as intended (i.e. teach-back) and evaluate progress. For patients, the collection of Insight Cards provided lasting access to their most meaningful rehabilitation experiences in their personal environment.

VBG consisted of a worksheet that facilitated the formulation of meaningful goals. The first part of the worksheet prompted patients to identify important personal values. The second part consisted of a prespecified algorithm to formulate desirable and feasible goals that were related to one of the identified values and could be attained within 6 months. Patients were also encouraged to set up calendar reminders and organize social support. In the third part of the worksheet, patients could plan their goal-directed activities in multiple steps, which facilitated gradual progress toward the goal. For each step, patients indicated what, when, and where as they planned the activity. Next, patients were prompted to identify potential barriers and formulate adequate strategies to overcome these barriers. When the first step was completed, patients could plan consecutive steps until the goal was attained. By continuously using the same step-by-step sequence, patients learned to set desirable and feasible goals for themselves and progress toward attainment through achievable steps, while anticipating potential barriers (Supplement 2). Supplement 3 provides an overview of the prototype intervention components and behavior change techniques (BCTs), according to the BCT taxonomy V1 of Michie and colleagues (Michie et al., 2013).

Both strategies were presented in the prototype workbook. This end result of the co-design project was not only based on cocreation and user experience evaluations but also informed by behavior change theory (Hermsen et al., 2014; Michie et al., 2013). To optimize the fit between the prototype intervention and the needs of each patient, we developed three workbook versions. Two workbook versions contained either VBG or Insight Cards. The third workbook version contained both strategies.

\section{Procedure}

We wanted to minimize the impact of this study on existing treatment procedures to resemble a real-world situation as much as possible. Therefore, we did not prescribe when, how, or how much the workbook should be used. Rather, treatment teams were free to select the appropriate patient and time point for introducing any of the workbook versions. For each participating treatment team, a 1-hour training session was provided in which the content of the workbook and suggestions for integrating the strategies into the treatment program were explained (e.g., by encouraging patients to use an Insight Card to express a particular relevant treatment experience). Patient inclusion was permitted at any time during treatment, as long as the HCPs considered the workbook to be of potential additional 
value. We recommended HCPs discuss the workbook with patients on a weekly basis during treatment, but they were free to decide how and when to use it. For a period of 6 months, printed workbooks were made available to the teams.

When the treatment staff decided to introduce the workbook, patients were approached by their physician or therapist who explained the study purpose and provided an information letter that explained the purpose of the study. Patients who were willing to participate signed an informed consent form and were provided with instructions on how to use the workbook by a member of the treatment staff.

\section{Data Collection}

Patient Interviews. Approximately 1 month post-IMPT, participants were contacted for a semistructured telephone survey of approximately 20 minutes by SE (male, physical therapist psychologist and as researcher involved in the development of the prototype intervention). The researcher was not involved in the treatment program, and the telephone survey was the first contact with the participants. In the introduction, the researcher explained the study aim and his role in this project. He also encouraged patients to speak frankly and provide all information that could be relevant for future use or development. Patients were asked to describe (1) the frequency of using the workbook, (2) the effect of the workbook on treatment adherence, (3) their satisfaction with using the workbook, (4) the contribution of the workbook to positive behavior change, and (5) its overall usability. Each topic was introduced with an open question (e.g., "To what extent does the workbook facilitate the maintenance of treatment goals at this moment?") and followed up with one probing question (e.g., "Could you give an example of how the workbook facilitated the maintenance of treatment goals?"). After each answer, the interviewer repeated the notes to check if they accurately reflected the meaning of the patient's responses. In response to our request to participate in an in-depth follow-up interview of 60 to 90 minutes to provide information about using the workbook over a prolonged follow-up time (approximately 10 months posttreatment), two participants agreed. We used a general interview guide approach to ensure that each topic of interest would be covered while adopting a flexible and responsive attitude to the participant feedback (Hermsen et al., 2016). The topic list queried experiences with the workbook during and after treatment, suggestions for improvement with respect to layout and content, and ideas for integrating the workbook in the treatment program. We developed interview guides for both interviews, each containing a topic list with guiding questions and a list of procedures (e.g., testing the recorder). The topic lists contained introductory questions to build rapport and make participants comfortable with the topic, key questions that focused on obtaining the information of interest, and ending questions to check if anything of relevance was missed. 
Focus Group. Four members of the rehabilitation team in Maastricht who were experienced with using the workbook participated in a 90 minute focus group session. This was held 12 weeks after the experimental phase and moderated by two researchers (AK/ $\mathrm{JP}$, both male, $\mathrm{PhD}$, physical therapists and as researchers involved in the development of the workbook). Two analysts (SE/SB) were present to take notes and record the session. Similar to the interviews, we developed a focus group guide that included procedures, task assignments, and 9 open-ended questions (Turner, 2010) (Supplement 4). During the session, the moderator ensured that all participants had sufficient opportunity to express their thoughts and ask clarifying questions when necessary. Each question concluded with a short summary before the group moved on to the next question.

\section{Data Analysis}

The dataset for the qualitative analysis consisted of verbatim transcripts of the focus groups and patient interviews and the notes that were taken during the telephone surveys. All files were imported into Atlas.ti version 8.4 (Scientific Software Development $\mathrm{GmbH}$ ) for analysis. We adopted a deductive thematic approach to identify, analyse, organize, describe, and report the themes that we found within our data (Braun \& Clarke, 2006; Nowell et al., 2017; Vaismoradi et al., 2013). Importantly, thematic analysis enables researchers to summarize the most important topics of a dataset using a stepwise approach that involves coding all data segments relevant to the research question. We constructed a deductive framework a priori that consisted of 3 themes we believed to be essential for determining the feasibility of the prototype intervention. We considered a theme as a meaningful group of data segments representing a phenomenon of interest in relation to the study question (Braun \& Clarke, 2006; Nowell et al., 2017; Vaismoradi et al., 2016). Applicability (theme 1) refers to the extent and manner in which the workbook could be integrated in the existing treatment program (Burchett et al., 2011). Acceptability refers to the extent by which the workbook is evaluated as suitable, satisfying, or attractive (Elbers et al., 2021; Proctor et al., 2011). We were not only interested in how participants judged the acceptability of the workbook content (theme 2), but also they evaluated the presentation form (theme 3). We added this latter theme because the current presentation form was chosen for practical purposes and we remained interested in alternative ideas.

In the first step of the data analysis, researchers read the data several times. All potentially relevant segments were coded according to these broad themes, but we allowed the possibility of adding extra themes if that would lead to a more accurate insight into the feasibility of this prototype intervention. In the second step, we inductively organized the data segments into subthemes to accurately describe the content (Nowell et al., 2017). 
SE and AK independently analysed the patient data, and SE and JP independently analysed the HCP focus group transcripts. Each step contained several iterations where researchers discussed the meaning of the data as well as how to accurately describe the data in terms of themes and subthemes with respect to the study aims. At the end of this process, the researchers held a final consensus meeting that involved summarizing the data in themes, subthemes, related quotes, and interim conclusions.

Although the answers on key questions during the interviews and focus groups were summarized by the interviewer to confirm they sufficiently captured their experience or opinion, participants were not involved in checking the results of the data analysis.

\section{RESULTS}

\section{Demographics}

During the course of the study, a workbook was offered to 19 patients; 8 patients did not respond to our requests to participate in the telephone survey. Therefore, our final dataset came from a focus group with 4 HCPs, telephone interviews with 11 patients, and indepth interviews with 2 of these patients. The HCPs were a behavior therapist (male, 19 years' experience), physiotherapist (male, 5 years' experience), behavior therapist (female, 14 years' experience), and occupational therapist (female, 11 years' experience). Table 1 provides an overview of the patient participant characteristics.

Table 1. Overview of Participant Characteristics $(\mathrm{n}=11)$.

\begin{tabular}{lc}
\hline Characteristics & Value \\
\hline Female, $\mathrm{n}(\%)$ & $6(55)$ \\
Age in years, mean (SD) & $55.20(12.21)$ \\
Pretreatment level of disability (PDI $\left.{ }^{\mathrm{a}}\right)$, mean (SD) & $35.29(9.48)$ \\
Pretreatment level of anxiety (HADS $)$, mean (SD) & $8.83(3.55)$ \\
Pretreatment level of depression (HADS), mean (SD) & $8.00(4.52)$ \\
\hline
\end{tabular}

Notes: aPDI: Pain Disability Index; ${ }^{\mathrm{H} H A D S}$ : Hospital Anxiety and Depression Scale.

The majority of codes could be clustered within our predefined framework, but we added the theme adaptation for a better fit. This theme covered the extent to which the prototype intervention could be adapted to each patient or whether personal characteristics were required for effective use. Table 2 provides an overview of our final coding scheme. 
Table 2. Coding Scheme, Including Themes, Subthemes, and Representative Quotations.

\begin{tabular}{|c|c|}
\hline Themes and subthemes & Representative quotations \\
\hline \multicolumn{2}{|c|}{ 1. Applicability of the workbook in the existing treatment programs } \\
\hline $\begin{array}{l}1.1 \text { Introduction of the intervention (48 } \\
\text { quotations) }\end{array}$ & $\begin{array}{l}\text { I would have preferred the assistance of a therapist with the } \\
\text { formulation of my first value-based goal. Without help, it took } \\
\text { me some time to understand the logic behind the procedure. [P8 }\end{array}$ \\
\hline $\begin{array}{l}\text { 1.2 Interaction with the treatment program } \\
\text { ( } 35 \text { quotations) }\end{array}$ & The workbook fits the treatment program well. [P11] \\
\hline 1.3 Final phase of treatment (7 quotations) & $\begin{array}{l}\text { I liked to review the Insight Cards during the follow-up session. } \\
\text { One patient even attached photos to his cards. It was nice to } \\
\left.\text { browse through and to gain insights in his experiences. [T2 }{ }^{\mathrm{b}}\right]\end{array}$ \\
\hline $\begin{array}{l}1.4 \text { Role of health care provider ( } 28 \\
\text { quotations) }\end{array}$ & $\begin{array}{l}\text { I liked the support of the therapists. During the sessions, we } \\
\text { went through the workbook and discussed everything. They also } \\
\text { reminded me sometimes to write down experiences. [P3] }\end{array}$ \\
\hline
\end{tabular}

\section{Acceptability of the workbook content}

2.1 Usage (33 quotations)

2.2 Action mechanisms (47 quotations)

2.3 Reported outcomes (30 quotations)

2.4 New components (27 quotations)
I did not actively use the workbook posttreatment, but it is nice to have it as a work of reference. [P17]

The workbook was an essential element in the process of learning to understand my condition. [P2]

For me, the workbook functioned as an extension of the treatment. I could see the program evolve, and patterns change. I could read back my personal development. [P8]

Specific information on triggers that could cause a relapse is not provided in the current prototype. [T3]

\section{Acceptability of the workbook form}

3.1 Type of delivery (mobile health vs paper and pencil; 32 quotations)

A negative point for both is that the intervention content would better fit an eHealth intervention in the current time. [T2]

3.2 Written instructions (18 quotations) Related to Value-Based Goal Setting: I believe the concept [of values] is difficult. For example, one patient did not understand the idea behind "source of inspiration." People that you needed to look up to...she found that a scary idea. [T4]

3.3 Appearance (13 quotations) I had difficulty with the initial structure of the layout. How does that work? [P8]

\section{Adaptability}

4.1 Personal characteristics (45 quotations) To use the workbook appropriately, patients will need some sort of self-reflecting skills, as well as intrinsic motivation. [T1]

Notes: ${ }^{\mathrm{P}}$ : patient; ${ }^{\mathrm{b}} \mathrm{T}$ : health care provider. 


\section{Applicability of the Workbook in Existing Treatment Programs}

Based on their own experiences, patients and HCPs discussed how the workbook could be optimally implemented and adopted throughout the treatment program. Regarding the introduction of the workbook, there was no consensus among HCPs about when to introduce the workbook during treatment. An early introduction of VBG was believed to facilitate the formulation of treatment goals at the start of the program, and Insight Cards were considered useful to immediately capture important experiences at the start of the program (e.g., the initial experiment of exposure in vivo). On the other hand, HCPs were hesitant to add more instruction time to an already information-dense start of the program.

If you provide patients with the workbook at the start of the program, you will also have to educate them on how it should be used... Yes, this will add to an already long queue of things that require explanation. [T2]

From the patients' perspective, an early introduction did not seem crucial: patients who received the workbook in later stages still evaluated its use as relevant.

Although patients reported substantial variation on time spent on the workbook during treatment sessions ranging from discussing the workbook during each contact to no integration at all, HCPs found Insight Cards easy to integrate into their therapy sessions. The interaction with the treatment program was more difficult for VBG.

Sometimes, value-based goals relate to higher order goals than the goals we can work with. If you start discussing patients'values, you can fill plenty of sessions. However, this will be a different type of treatment than we are currently providing. [T1]

In general, HCPs commented that regular checks of the content were preferable within treatment contact time but that the total time spent should be limited. Both patients and HCPs specifically considered these checks important during the final phase of treatment. For example, a patient commented on whether it would be relevant to evaluate the workbook during the final phase of treatment.

Yes, absolutely. That makes sense to me. It is not necessary to discuss the workbook every week, but it would help to ask at certain moments how things are going. Then, patients can show how they are using the workbook. They might be using it improperly. That sometimes by evaluating and also discuss this during the final evaluation: what's the status? How far did you come? [P2] 
Patients reported mixed experiences relating to the role of the health care provider regarding the workbook. Active involvement was considered useful as it facilitated the transition from the workbook to treatment and vice versa. Patients who did not actively discuss the workbook with their HCPs believed that more involvement would have led to better outcomes. As a minimum, they recommended an HCP-led introduction where the use of the workbook would be explained.

\section{Acceptability of the Workbook Content}

Patients and HCPs reflected on what potential action mechanisms were involved and which behavioral outcomes were targeted. Participants reported that the use of Insight Cards helped to create a moment of reflection on important experiences. Also, rereading the experiences provided a better understanding of important treatment concepts. For VBG, patients indicated that it contributed to pacing of activities, planning meaningful goals, and anticipating the effort needed to attain the goals. One patient who used the VBG strategy to plan a long journey in advance commented.

A positive experience is ... Normally it is just persisting, no matter what. I will do this now. But here, if you aim for greater things, you will need to work towards it. I have seen that clearly now. [P8]

Patients reported a shift from active use during treatment to passive use (as a work of reference) posttreatment.

I have not actively used the workbook after treatment, but I am glad that it is available as a reference book. [P17]

During active use, VBG especially was considered time-consuming, which hindered regular use for some patients. In the posttreatment phase, one patient reported that reading the workbook content helped him counter an impending relapse. Participants made multiple suggestions for new components to the prototype intervention such as additional reading material on pain education, a specific section that describes the possibility of relapse, and the option of prioritizing the most important Insight Cards. Participants further suggested discussing the workbook with peers during group meetings and made specific suggestions should the workbook be developed into a digital app, such as adding informative video clips and a digital avatar that could interact with the patient.

Participants reported that the workbook contributed to facilitating the pursuit of meaningful goals, providing a structure to the treatment process, pacing activities, monitoring progress, and revealing the most important milestones during the program. 
A patient started with the Insight Cards at the final treatment phase. Nevertheless, during the refresher day his workbook was an exemplar of how they [the Insight Cards] should be used. Completely filled out and illustrated with photographs. He also mentioned that, in case of potential relapse, he could imagine himself using the workbook and browsing through his experiences. [T2]

These positive outcomes were not shared by all participants. Some patients reported that the intention of the prototype intervention was not clear or questioned its efficacy.

I wonder whether a workbook would be sufficient to ensure the transfer to the home situation. [P13]

Also, HCPs were cautious that too much emphasis on personal values could cause patients to focus on topics and goals that were beyond the scope of the treatment.

\section{Acceptability of the Workbook Form}

The general appearance and structure was appealing to most participants, but some patients provided suggestions for reordering the workbook and moving all instructions to the beginning. For VBG, the written instructions were considered too elaborate and complex, which caused confusion and problems in understanding.

It [the workbook instructions] should be easier to read. It may be due to my short attention span or because I am not a good reader, but I did like the underlying idea. [P9]

The instruction text for the Insight Cards was shorter and easier to understand. Participants indicated that the included examples were helpful for both relapse prevention strategies.

Overall, participants would prefer a mobile health (mHealth) app over a printed workbook as type of delivery. In particular, the possibility of combining the strategies with smartphone functionalities such as a calendar and camera could lead to more personalized experiences and goals. Moreover, a digital app would be accessible throughout the day, allowing patients to directly record experiences, browse through insights, or plan new goals.

I would prefer an app. An app can provide feedback and is able to alert you. For example, I will plan a $3 k$ walk for tomorrow. Then I will receive a reminder that I should go for a $3 k$ walk. If think "no! not tomorrow," then-it is quite simple-I will modify the calendar... this is the future.. I have not grown up with this thing [mobile phone]. But nowadays people only look on their phones throughout the day. They will benefit more from this [the app] than from this paper [the workbook]. So I would think that is very important. [P8] 


\section{Adaptability}

The theme adaptability was added in response to multiple comments relating to individual personal characteristics that were believed to either facilitate or hinder optimal use of the workbook. In particular, participants reported that the current version of the prototype intervention required a high level of commitment and an active mindset to autonomously explore the features of the workbook.

Because of my work, I am used to discover things on my own, but I expect that this method will not work for everyone if it is not clearly instructed. [P8]

Patients with low health literacy were expected to encounter problems with the current amount of instruction texts, reading level, writing down their own input, and analyzing which values would underlie their most important treatment goals. Characteristics that were reported to facilitate the use of the workbook included being organized, being able to reflect on experiences, and possessing adequate reading and writing skills.

\section{DISCUSSION}

\section{Principal Findings}

This study was conducted to assess the feasibility of two relapse prevention strategies specifically designed to enhance IMPT programs. Overall, the workbook was perceived by participants as useful, easy to use, and in line with the treatment program. However, there was a difference in how the individual relapse prevention strategies were perceived. Insight Cards were expected to benefit all patients and were relatively easy to learn and apply. VBG helped patients to plan meaningful goals, but these were more difficult to understand and did not always fit into the treatment program. However, it is important to note that the focus group consisted solely of HCPs who provided exposure in vivo treatment and were not experienced with VBG. Participants indicated a preference for a digital app over a paper-and-pencil workbook as a future delivery mode. Other suggestions for improvement included more specific implementation guidelines for the treatment staff, group sessions among patients to discuss their input, and more attention to the workbook during the final phase of the treatment. Overall, these findings indicate that the workbook is feasible within the context of IMPT and acceptable to both patients and HCPs.

Importantly, these initial results contain detailed feedback on how the strategies can be refined. First, the study protocol allowed for substantial variation in when and how the workbook was applied. This flexible approach maximized HCP autonomy with respect to dose and content but at times resulted in limited or even no interaction at all once the 
workbook was introduced. As a consequence, not all intervention components were used effectively by all participating patients. For example, HCPs could use the Insight Cards to check if the patient understood important treatment principles as intended, but the dataset includes no mention of such a teach-back occurrence. Therefore, we believe that, in line with other study findings, an extensive onboarding procedure with additional guidelines, examples, and training sessions would improve overall implementation and optimize the potential of the workbook (Chan et al., 2017; Kaipainen et al., 2017; Pope et al., 2018). Based on the evaluations in this study regarding patient characteristics and requirements for optimal use, this onboarding procedure could also contain a deliberate consideration whether either or both interventions may benefit a patient. Second, the VBG sequence needs revision to improve clarity for patients and ensure that the goal-setting procedure matches the treatment program. The sequence was based on the insight that value-based goal-setting procedures outperformed specific, measurable, achievable, relevant and time bound (i.e. SMART) goal setting (Chase et al., 2013; Höchli et al., 2018). However, patients reported difficulties in understanding the concept of values through written instructions in the workbook, particularly when the treatment program did not structurally include a values assessment. Altering the VBG procedure to shift the initial emphasis from values to goals may increase clarity; patients could begin formulating specific goals related to improved physical functioning instead of starting with personal values. Subsequently, assessing why this particular goal is relevant to the patient could direct attention toward associated values. Third, HCP responses concerning the amount of time spent with the prototype intervention suggests that they experienced a trade-off between focusing on the treatment program or preventing relapse with the workbook. This indicates that relapse prevention with the current version of the workbook is not yet perceived as an integral aspect of the treatment. Given that the workbook was introduced as an addition to the existing IMPT, this finding is not surprising. Nevertheless, future development should take time efficiency into account and focus on increased integration of the relapse prevention strategies into the existing treatment protocols. One possibility is to relate the identification of problems in daily life functioning during the assessment phase (e.g., by using instruments such as the Canadian Occupational Performance Measure) to the goalsetting procedure of VBG. Furthermore, integration of Insight Cards into clinical practice could be enhanced by routinely relating this to specific communication strategies, such as a teach-back approach (Feinberg et al., 2019; Klingbeil \& Gibson, 2018). Reflecting on Insight Cards during patient-therapist conversations could facilitate both shared decision making and teach-back and empower patients to actively participate.

One promising direction for the development of the prototype is to embed these strategies in an mHealth app (i.e. software apps designed for mobile devices to provide or support health care services) (Silva et al., 2015). This domain is becoming increasingly important 
in the assessment and treatment of chronic pain and is particularly suited for tailoring specific strategies to individual needs and preferences (Keogh et al., 2010). With machine learning approaches, it is even possible to automate the process of personalizing the strategies based on user-generated data (Rabbi et al., 2018). Another advantage of mHealth is the opportunity of letting both strategies interact. For example, if a patient used an Insight Card to highlight an effective strategy to overcome barriers to physical activity, this card could also be used as a future solution to anticipated problems within the planning procedure of VBG. Although the idea of a digital intervention had already been suggested by stakeholders in earlier development stages, we did not make any decisions on its final form prior to this feasibility study. Because our study findings are in accordance with these earlier suggestions, we believe there is potential in transferring this prototype workbook into an mHealth app.

\section{Limitations}

Limitations of this study include a small sample size and relatively high dropout rate. Because the study was designed, conducted, and analysed by the same three researchers, who were also involved in the development of the workbook, confirmation bias and socially desirable responses may have resulted. Furthermore, due to organizational reasons at location Hoensbroek directly after the inclusion period, we were only able to collect evaluations from HCPs from location Maastricht, where regular reflections on patients' values are beyond the scope of their treatment program. With these limitations in mind, it is important to reflect on the validity of the conclusions of this study. Concerning the adequacy of the sample, Malterud and colleagues (Malterud et al., 2016) have introduced the concept of information power, which is determined by five factors: narrow or broad study aim, sample specificity, established underlying theory, quality of dialogue, and type of analysis strategy. We believe that the specific focus in our study objective on 3 key factors for feasibility positively contributed to the information power of this dataset. Furthermore, all participants that we interviewed received instructions to use the workbook, participated in an IMPT program, and had-at minimum-made an effort to use the workbook in this setting, which not only resulted in high specificity but also to a high quality of dialogue. In addition, we included several established procedures to enhance the credibility of our findings and minimize bias, including member checks, triangulation of researchers and data sources, and including questions regarding negative experiences with the workbook to search for disconfirming evidence (Carter et al., 2014; Creswell \& Miller, 2000; Nowell et al., 2017). However, we conducted a cross-case analysis and the low sample size resulted in limited variation on personal characteristics and a low likelihood that potential problems in use did occur within the sample (Malterud et al., 2016; Viechtbauer et al., 2015), which limits the generalizability of our findings. 
Although multiple reasons could have contributed to these limitations, an important factor may have been our real-world approach toward the use of the prototype intervention within the inclusion period. We expected that the active participation of the treatment teams during previous development phases would contribute to high patient inclusion rates. However, it is likely that the limited guidance on when or how to explain the workbook and absence of fixed procedures regarding patient recruitment increased the required effort for HCPs to integrate this study into their treatment routine. Although this means that the extent to which this workbook can work in IMPT programs is inconclusive, we did obtain important insights for further developing the prototype intervention. From an intervention design perspective, the feedback from actual use within the intended environment is crucial to further refine the strategies and adapt them to that specific context ( $\mathrm{O}^{\prime} \mathrm{C}$ athain et al., 2015). Regarding the low response rate on the in-depth interviews, some patients indicated that they already provided a full evaluation of the workbook in the telephone interview. Other patients mentioned the traveling distance as main reason. In addition, we believe that a moment of direct contact with the researchers prior to the telephone interview could have helped to better explain the importance of the interview and establish a good rapport in advance. For these reasons, this study should be regarded as the first iteration in the overall process of transforming a prototype into an effective intervention for clinical practice. Czajkowski et al (2015) emphasize the need for initial prototyping before conducting more stringent tests in order to first align the behavioral strategies to the clinical context in which they will be implemented. In addition, experimental medicine highlights the need for a stepwise approach toward intervention development. This framework consists of multiple subsequent steps that should be undertaken to examine the relationships between the intervention and its effect on physical functioning and the modifiable behavioral factors that mediate this relationship (Sheeran et al., 2017). Consequently, further development and testing are required and should indicate whether these strategies lead to a change in specific health behaviors such as goal setting and problem-solving and to what extent this change causes clinically relevant long-term improvements for patients with chronic pain. In addition, these limitations provide valuable information in preparation of future trials, including more emphasis on training HCPs in how to use the strategies, more integration of study procedures within clinical practice, and improved patient fidelity procedures to decrease dropout.

\section{Conclusion}

This first test of the relapse prevention workbook in a real-world setting of IMPT programs resulted in important insights regarding form, content, and use, as well as its interaction with the treatment program and study design. Although these initial results indicate a favorable evaluation of behavior regulation strategies within the workbook, this study 
encountered multiple barriers regarding implementation and data collection that limit the generalizability of these results. Future studies should address the fidelity of HCPs and patients and should include clear procedures regarding recruitment and use of both relapse prevention strategies during treatment. Future development efforts should consider eHealth or mHealth options, extensive onboarding, and a modified value-based goalsetting procedure for the VBG strategy.

\section{Acknowledgments}

Proofreading by Les Hearn (Scientific Proofreading and Editing). This work was supported by a Regionale Aandacht en Actie voor Kenniscirculatie grant from the Regieorgaan Stichting Innovatie Alliantie (SIA RAAK 2014-01-23P).

\section{Conflicts of Interest}

None declared.

\section{Abbreviations}

BCT: behavior change technique; HCP: health care provider; IMPT: Interdisciplinary multimodal pain therapy; mHealth: mobile health; SMART: specific, measurable, achievable, relevant and time bound; VBG: Value-Based Goal Setting. 


\section{REFERENCES}

Adams, L., \& Turk, D. (2015). Psychosocial Factors and Central Sensitivity Syndromes. Current Rheumatol Rev, 11(2), 96-108. https://doi.org/10.2174/1573397111666150619095330

Bowen, D. J., Kreuter, M., Spring, B., Cofta-Woerpel, L., Linnan, L., Weiner, D., Bakken, S., Kaplan, C. P., Squiers, L., Fabrizio, C., \& Fernandez, M. (2009). How We Design Feasibility Studies. Am J Prev Med, 36, 452-457. https:// doi.org/10.1016/j.amepre.2009.02.002

Braun, V., \& Clarke, V. (2006). Using thematic analysis in psychology. Qual Res Psychol, 3(2), 77-101. https://doi. org/10.1191/1478088706qp063oa

Burchett, H., Umoquit, M., \& Dobrow, M. (2011). How do we know when research from one setting can be useful in another? A review of external validity, applicability and transferability frameworks. J Health Services Res Pol, 16(4), 238-244 https://doi.org/10.1258/jhsrp.2011.010124

Carter, N., Bryant-Lukosius, D., Dicenso, A., Blythe, J., \& Neville, A. J. (2014). The use of triangulation in qualitative research. In Onc Nur For, 41(5), 545-547. https://doi.org/10.1188/14.ONF.545-547

Chan, S., Godwin, H., Gonzalez, A., Yellowlees, P. M., \& Hilty, D. M. (2017). Review of Use and Integration of Mobile Apps Into Psychiatric Treatments. Curr Psych Rep, 19, 96. https://doi.org/10.1007/s11920-017-0848-9

Chase, J. A., Houmanfar, R., Hayes, S. C., Ward, T. A., Vilardaga, J. P., \& Follette, V. (2013). Values are not just goals: Online ACT-based values training adds to goal setting in improving undergraduate college student performance. J Context Behav Sci, 2(3-4), 79-84. https://doi.org/10.1016/j.jcbs.2013.08.002

Creswell, J. W., \& Miller, D. L. (2000). Determining validity in qualitative inquiry. Theory Pract, 39(3), 124-130. https:// doi.org/10.1207/s15430421tip3903_2

Czajkowski, S. M., Powell, L. H., Adler, N., Naar-King, S., Reynolds, K. D., Hunter, C. M., Laraia, B., Olster, D. H., Perna, F. M., Peterson, J. C., Epel, E., Boyington, J. E., \& Charlson, M. E. (2015). From ideas to efficacy: The ORBIT model for developing behavioral treatments for chronic diseases. Health Psychol, 34(10), 971-982. https://doi. org/10.1037/hea0000161

den Hollander, M., Goossens, M., de Jong, J., Ruijgrok, J., Oosterhof, J., Onghena, P., Smeets, R., \& Vlaeyen, J. W. S. (2016). Expose or protect? A randomized controlled trial of exposure in vivo vs pain-contingent treatment as usual in patients with complex regional pain syndrome type 1. Pain, 157(10), 2318-2329. https://doi. org/10.1097/j.pain.0000000000000651

de Ridder, D., Geenen, R., Kuijer, R., \& van Middendorp, H. (2008). Psychological adjustment to chronic disease. Lancet, 372(9634), 246-255. https://doi.org/10.1016/S0140-6736(08)61078-8

DiMatteo, M. R. (2004). Variations in patients' adherence to medical recommendations: A quantitative review of 50 years of research. Med Care, 42(3), 200-209, https://doi.org/10.1097/01.mlr.0000114908.90348.f9

Elbers, S., Gessel, C. van, Renes, R. J., Lugt, R. van der, Wittink, H., \& Hermsen, S. (2021). Innovation in pain rehabilitation using co-design methods during the development of a relapse prevention intervention: Case study. J Med Int Res, 23(1). https://doi.org/10.2196/18462

Feinberg, I., Ogrodnick, M. M., Hendrick, R. C., Bates, K., Johnson, K., \& Wang, B. (2019). Perception versus reality: The use of teach back by medical residents. Health Lit Res Pract, 3(2), e117-e126. https://doi.org/10.3928/2474830720190501-01

Gatchel, R. J., McGeary, D. D., McGeary, C. A., \& Lippe, B. (2014). Interdisciplinary chronic pain management. Am Psychol, 69(2), 119-130. https://doi.org/10.1037/a0035514

Hermsen, S., Renes, R. J., \& Frost, J. (2014). Persuasive by Design: a model and toolkit for designing evidencebased interventions. In J. van Leeuwen, P. J. Stappers, M. H. Lamers, \& M. J. M. R. Thissen (Eds.), Creating 
the Difference: Proceedings of the Chi Sparks 2014 Conference (pp. 74-77). The Hague University of Applied Sciences. http://proceedings.chi-sparks.nl

Hermsen, S., van der Lugt, R., Mulder, S., and Renes, R. (2016) How I learned to appreciate our tame social scientist: experiences in integrating design research and the behavioural sciences, in Lloyd, P. and Bohemia, E. (eds.), Future Focused Thinking - DRS International Conference 2016, 27 - 30 June, Brighton, United Kingdom. https://doi.org/10.21606/drs.2016.17

Höchli, B., Brügger, A., \& Messner, C. (2018). How focusing on superordinate goals motivates broad, long-term goal pursuit: A theoretical perspective. Front Psychol, 9, 1879. https://doi.org/10.3389/fpsyg.2018.01879

Hollander, M. den, de Jong, J. R., Volders, S., Goossens, M. E., Smeets, R. J., \& Vlaeyen, J. W. (2010). Fear reduction in patients with chronic pain: A learning theory perspective. Exp Rev Neurother, 10(11), 1733-1745. https://doi. org/10.1586/ern.10.115

Kaipainen, K., Välkkynen, P., \& Kilkku, N. (2017). Applicability of acceptance and commitment therapy-based mobile app in depression nursing. Trans Beh Med, 7(2), 242-253. https://doi.org/10.1007/s13142-016-0451-3

Kaiser, U., Treede, R. D., \& Sabatowski, R. (2017). Multimodal pain therapy in chronic noncancer pain-gold standard or need for further clarification? Pain, 158(10), 1853-1859. https://doi.org/10.1097/j.pain.0000000000000902

Kamper, S. J., Apeldoorn, A. T., Chiarotto, A., Smeets, R. J. E. M., Ostelo, R. W. J. G., Guzman, J., \& van Tulder, M. W. (2014). Multidisciplinary biopsychosocial rehabilitation for chronic low back pain. Cochrane Database of Syst Rev. https://doi.org/10.1002/14651858.CD000963.pub3

Keogh, E., Rosser, B. A., \& Eccleston, C. (2010). E-Health and chronic pain management: Current status and developments. Pain, 151(1), 18-21. https://doi.org/10.1016/j.pain.2010.07.014

Klingbeil, C., \& Gibson, C. (2018). The teach back project: A system-wide evidence based practice implementation. J Ped Nur, 42, 81-85. https://doi.org/10.1016/j.pedn.2018.06.002

Kwasnicka, D., Dombrowski, S. U., White, M., \& Sniehotta, F. (2016). Theoretical explanations for maintenance of behaviour change: a systematic review of behaviour theories. Health Psychol Rev, 10(3), 277-296. https://doi. org/10.1080/17437199.2016.1151372

Lee, W. L., Abdullah, K. L., Bulgiba, A. M., \& Zainal Abidin, I. (2013). Prevalence and predictors of patient adherence to health recommendations after acute coronary syndrome: Data for targeted interventions? Eur J Card Nur, 12(6), 512-520. https://doi.org/10.1177/1474515112470056

Malterud, K., Siersma, V. D., \& Guassora, A. D. (2016). Sample Size in Qualitative Interview Studies: Guided by Information Power. Qual Health Res, 26(13), 1753-1760. https://doi.org/10.1177/1049732315617444

Michie, S., Richardson, M., Johnston, M., Abraham, C., Francis, J., Hardeman, W., Eccles, M. P., Cane, J., \& Wood, C. E. (2013). The behavior change technique taxonomy (v1) of 93 hierarchically clustered techniques: Building an international consensus for the reporting of behavior change interventions. Ann Beh Med, 46(1), 81-95. https://doi.org/10.1007/s12160-013-9486-6

Miettinen, T., Kautiainen, H., Mäntyselkä, P., Linton, S. J., \& Kalso, E. (2019). Pain interference type and level guide the assessment process in chronic pain: Categorizing pain patients entering tertiary pain treatment with the Brief Pain Inventory. PLoS ONE, 14(8). https://doi.org/10.1371/journal.pone.0221437

Morley, S. (2008). Relapse prevention: Still neglected after all these years. Pain, 134(3), 239-240. https://doi. org/10.1016/j.pain.2007.12.004

Morley, S., \& Williams, A. (2015). New developments in the psychological management of chronic pain. Can $J$ Psychiatry, 60(4), 168-175. https://doi.org/10.1177/070674371506000403

Morley, S., Williams, A., \& Eccleston, C. (2013). Examining the evidence about psychological treatments for chronic pain: Time for a paradigm shift? Pain, 154(10), 1929-1931. https://doi.org/10.1016/j.pain.2013.05.049

Nicholas, M. K., Asghari, A., Corbett, M., Smeets, R. J. E. M., Wood, B. M., Overton, S., Perry, C., Tonkin, L. E., \& Beeston, L. (2011). Is adherence to pain self-management strategies associated with improved pain, depression 
and disability in those with disabling chronic pain? Eur J Pain, 16(1), 93-104. https://doi.org/10.1016/j. ejpain.2011.06.005

Noar, S. M., Benac, C. N., \& Harris, M. S. (2007). Does tailoring matter? Meta-analytic review of tailored print health behavior change interventions. Psychol Bull, 133(4), 673-693. https://doi.org/10.1037/0033-2909.133.4.673

Nowell, L. S., Norris, J. M., White, D. E., \& Moules, N. J. (2017). Thematic analysis: Striving to meet the trustworthiness criteria. Int J Qual Methods, 16(1). https://doi.org/10.1177/1609406917733847

O’Cathain, A., Hoddinott, P., Lewin, S., Thomas, K. J., Young, B., Adamson, J., Jansen, Y. J. F. M., Mills, N., Moore, G., \& Donovan, J. L. (2015). Maximising the impact of qualitative research in feasibility studies for randomised controlled trials: Guidance for researchers. Pilot Feasibility Stud, 1(32). https://doi.org/10.1186/s40814-0150026-y

Pope, L., Garnett, B., \& Dibble, M. (2018). Lessons learned through the implementation of an eHealth physical activity gaming intervention with high school youth. Games Health J, 7(2), 136-142. https://doi.org/10.1089/ g4h.2017.0164

Proctor, E., Silmere, H., Raghavan, R., Hovmand, P., Aarons, G., Bunger, A., Griffey, R., \& Hensley, M. (2011). Outcomes for implementation research: Conceptual distinctions, measurement challenges, and research agenda. Admin Pol Mental Health and Mental Health Serv Res, 38(2), 65-76. https://doi.org/10.1007/s10488-010-0319-7

Rabbi, M., Aung, M. S. H., Gay, G., Reid, M. C., \& Choudhury, T. (2018). Feasibility and acceptability of mobile phonebased auto-personalized physical activity recommendations for chronic pain self-management: Pilot study on adults. J med Int Res, 20(10). https://doi.org/10.2196/10147

Reuter, T., Ziegelmann, J. P., Lippke, S., \& Schwarzer, R. (2009). Long-term relations between intentions, planning, and exercise: A 3-year longitudinal study afer orthopedic rehabilitation. Rehabil Psychol, 54(4), 363-371. https://doi.org/10.1037/a0017830

Sheeran, P., Klein, W. M. P., \& Rothman, A. J. (2017). Health behavior change: Moving from observation to intervention. Ann Rev Psychol, 68, 573-600. https://doi.org/10.1146/annurev-psych-010416-044007

Silva, B. M. C., Rodrigues, J. J. P. C., de la Torre Díez, I., López-Coronado, M., \& Saleem, K. (2015). Mobile-health: A review of current state in 2015. J Biomed Informatics, 56, 265-272. https://doi.org/10.1016/j.jbi.2015.06.003

Steiner, J. F. (2012). Rethinking adherence. Ann Int Med, 157(8), 580-585. https://doi.org/10.7326/0003-4819-157-8201210160-00013

Turk, D. C., \& Rudy, T. E. (1991). Neglected topics in the treatment of chronic pain patients - relapse, noncompliance, and adherence enhancement. Pain, 44(1), 5-28. https://doi.org/10.1016/0304-3959(91)90142-K

Turner D. (2010) Qualitative interview design: a practical guide for novice investigators. Qual Rep, 15(3), 754-760.

Vaismoradi, M., Jones, J., Turunen, H., \& Snelgrove, S. (2016). Theme development in qualitative content analysis and thematic analysis. J Nurs Educ Pract, 6(5), 100-110. https://doi.org/10.5430/jnep.v6n5p100

Vaismoradi, M., Turunen, H., \& Bondas, T. (2013). Content analysis and thematic analysis: Implications for conducting a qualitative descriptive study. Nurs Health Sci, 15(3), 398-405. https://doi.org/10.1111/nhs.12048

Viechtbauer, W., Smits, L., Kotz, D., Budé, L., Spigt, M., Serroyen, J., \& Crutzen, R. (2015). A simple formula for the calculation of sample size in pilot studies. J Clin Epidem, 68(11), 1375-1379. https://doi.org/10.1016/j. jclinepi.2015.04.014

Wood, W., \& Rünger, D. (2016). Psychology of habit. Ann Rev Psychol, 67, 289-314. https://doi.org/10.1146/annurevpsych-122414-033417 


\section{SUPPLEMENTARY MATERIALS}

This chapter contains the following supplementary materials

- $\quad$ Supplement 1: Insight Cards

- Supplement 2:VBG

- Supplement 3 BCT overview

- $\quad$ Supplement 4: Focus group guide

- Supplement 5: Full workbook (Dutch Languague Version)

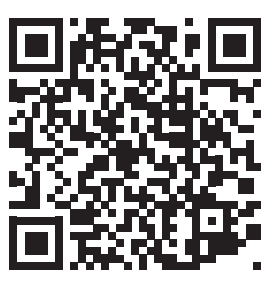

The supplementary materials can be accessed via this QR code or link https://github.com/stefanelbers/doctoral_thesis/ 



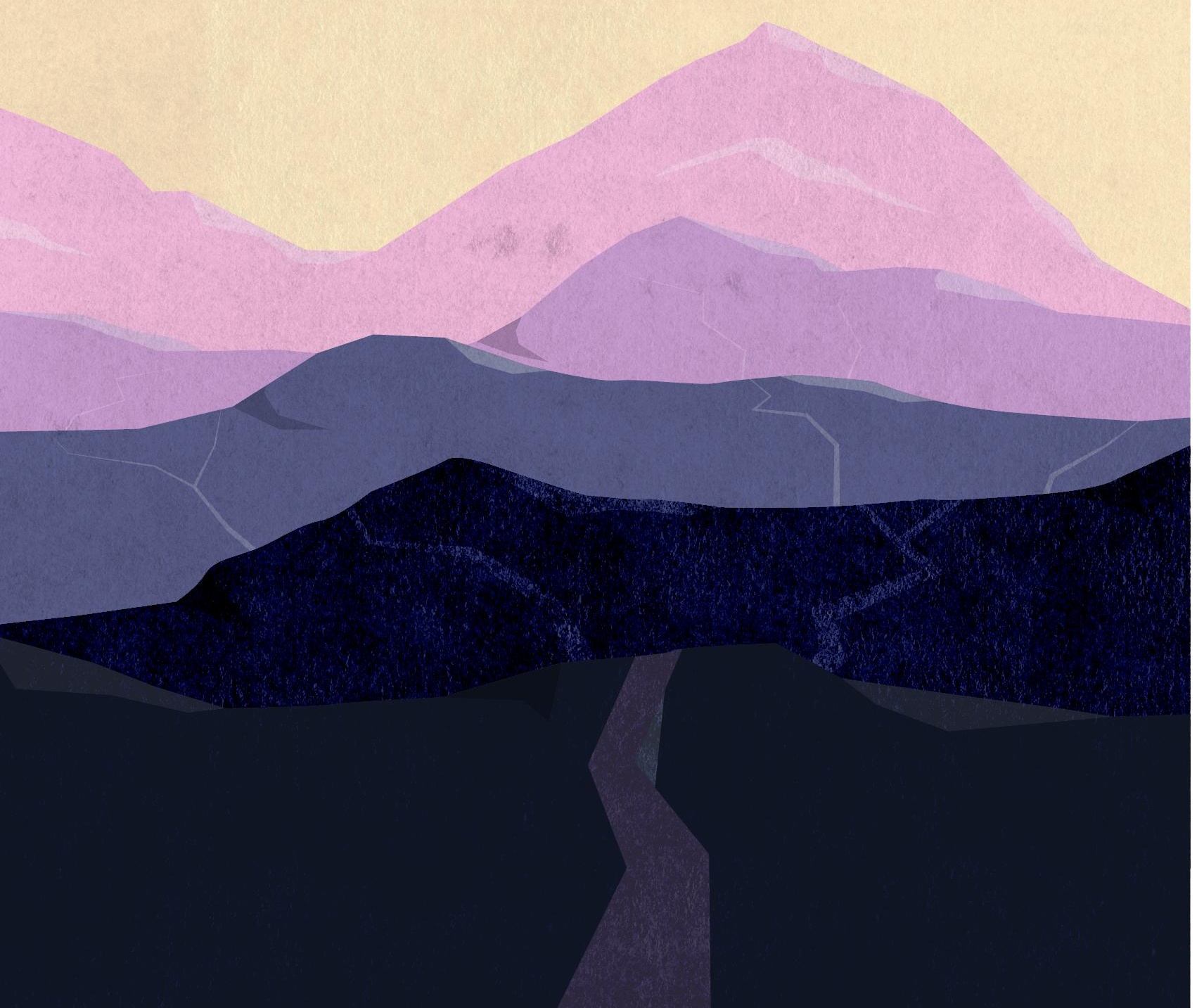




\title{
07
}

\section{Mobile Health App (AGRIPPA) to Prevent Relapse After Successful Interdisciplinary Treatment for Patients With Chronic Pain:} Protocol for a Randomized Controlled Trial

\author{
S. Elbers ${ }^{1,2}$, J. Pool ${ }^{1}$, H. Wittink ${ }^{1}$, A. Köke ${ }^{2,3,4}$, E. Scheffer ${ }^{1}$, R. Smeets ${ }^{2,5}$
}

JMIR Research Protocols (2020), 9(8), e18632

1. Lifestyle and Health Research Group, Healthy and Sustainable Living Research Centre, University of Applied Sciences Utrecht, Utrecht, The Netherlands.

2. Department of Rehabilitation Medicine, Faculty of Health, Life Sciences and Medicine, Maastricht University, Maastricht, The Netherlands.

3. Adelante Centre of Expertise in Rehabilitation and Audiology, Hoensbroek, The Netherlands.

4. South University of Applied Sciences, Heerlen, The Netherlands.

5. CIR Rehabilitation, Eindhoven, The Netherlands. 


\section{ABSTRACT}

Background: To facilitate adherence to adaptive pain management behaviours after interdisciplinary multimodal pain treatment, we developed a mobile health app (AGRIPPA app) that contains two behaviour regulation strategies.

Objective: The aims of this project are (1) to test the effectiveness of the AGRIPPA app on pain disability; (2) to determine the cost-effectiveness; and (3) to explore the levels of engagement and usability of app users.

Methods: We will perform a multicenter randomized controlled trial with two parallel groups. Within the 12-month inclusion period, we plan to recruit 158 adult patients with chronic pain during the initial stage of their interdisciplinary treatment progamme in one of the 6 participating centers. Participants will be randomly assigned to the standard treatment condition or to the enhanced treatment condition in which they will receive the AGRIPPA app. Patients will be monitored from the start of the treatment progamme until 12 months posttreatment.

In our primary analysis, we will evaluate the difference over time of pain-related disability between the two conditions. Other outcome measures will include health-related quality of life, illness perceptions, pain self-efficacy, app system usage data, productivity loss, and health care expenses.

Results: The study was approved by the local Medical Research Ethics Committee in October 2019. As of March 20, 2020, we have recruited 88 patients.

Conclusions: This study will be the first step in systematically evaluating the effectiveness and efficiency of the AGRIPPA app. After 3 years of development and feasibility testing, this formal evaluation will help determine to what extent the app will influence the maintenance of treatment gains over time. The outcomes of this trial will guide future decisions regarding uptake in clinical practice.

Trial Registration: Netherlands Trial Register NL8076; https:/www.trialregister.nl/ trial/8076

International Registered Report Identifier (IRRID): DERR1-10.2196/18632

Key words: telemedicine; chronic pain; recurrence; clinical trial protocol; rehabilitation; randomized controlled trial; cost-benefit analysis; treatment adherence and compliance; mobile apps; patient care team. 


\section{INTRODUCTION}

\section{Background and Rationale}

Chronic pain is a major contributor to worldwide disability, affecting approximately $20 \%$ of the global population (Breivik et al., 2006; Treede et al., 2015; Vos et al., 2012). For many patients, ongoing or recurrent pain severely impacts their physical, social, and mental health, as it interrupts ongoing activities and thereby continuously interferes with daily life functioning. Over time, this impacts patients' sense of self and quality of life (Vlaeyen et al., 2016).

In many cases, there is no monodisciplinary treatment available that can cure the persisting pain. Instead, the multifaceted nature of chronic pain, including biomedical as well as psychological and social factors, often requires a comprehensive treatment approach focusing on improving daily life functioning rather than reducing pain (Foster et al., 2018; Gatchel et al., 2014; Linton \& Shaw, 2011). To realize this, interdisciplinary multimodal pain therapy (IMPT) progammes have evolved that aim to support patients in learning to live a meaningful life irrespective of pain. These progammes share a biopsychosocial orientation toward chronic pain and often include both neuroscientific models of pain physiology as well as (cognitive) behavioural treatment principles (Gatchel et al., 2014; Morley \& Williams, 2015).

Although the effectiveness of IMPT progammes has been well established (Banerjee \& Argáez, 2017; Kamper et al., 2014; Morley et al., 2013; Schatman, 2012), maintaining the positive effect of the treatment on patients' daily lives over time remains a major challenge (Morley, 2008; Turk \& Rudy, 1991). The problem of relapse is not unique to the domain of pain treatment but has been observed across all health behaviour domains (e.g. Middleton et al., 2013; Miller, 2012). In response, many treatment progammes have added relapse prevention strategies that aim to preserve treatment gains over time (e.g. Fleig et al., 2013; Kwasnicka et al., 2016). In the context of chronic pain treatment, examples of such strategies include self-practice exercises (Silvemark et al., 2014; Smeets et al., 2008), booster sessions (Mangels et al., 2009; Tavafian et al., 2011), or encouragement of patients to take notes during treatment (Monticone et al., 2016; Persson et al., 2012). However, the integration of these particular strategies within the treatment progamme as well as an underlying theoretical rationale regarding how it may prevent relapse are often not described in clinical studies. Moreover, the effectiveness of these behaviour regulation strategies remains unknown because they are usually evaluated as a part of the full progamme or with a limited follow-up period. 


\section{Mobile Health}

The emergence of mobile health (mHealth) provides new opportunities to support behaviour regulation to maintain or enhance the long-term treatment effect of IMPT progammes. Despite substantial variations concerning study quality, interventions, and outcomes, mHealth apps are generally regarded as a promising strategy to facilitate adherence to treatment principles or to increase self-management skills (e.g. Anglada-Martinez et al., 2015; Rathbone \& Prescott, 2017). A specific advantage is that an app can include multiple interacting behaviour regulation strategies within its digital environment (e.g., automatically linking personalized goal setting regarding physical activity to accelerometer output). Moreover, mHealth strategies can integrate other smartphone functionalities such as digital calendars, instant messaging services, or a camera, thereby offering personalized behaviour regulation strategies that support the transfer of treatment insights into each patient's personal environment.

Despite the potential and current popularity of mHealth apps, the effectiveness on health-related outcome measures varies greatly (Byambasuren et al., 2018). Factors such as engagement-defined as the extent of app use as well as the corresponding subjective experience (Perski et al., 2018)-and usability-defined as the relative ease with which users can use an app to achieve a particular goal (Coursaris \& Kim, 2011; Zapata et al., 2015)-may account for this variability (Byambasuren et al., 2018; Zapata et al., 2015). For example, patients that use an app to change their health behaviours may use the app in a different way than intended or stop using the app after several days, which prevents facilitating the intended behaviour change (Perski et al., 2018). Therefore, evaluations concerning the effectiveness and clinical importance of mHealth apps on health outcomes should take evaluations on user engagement and perceived usability into account (Pham et al., 2019; Zapata et al., 2015).

\section{Previous Studies}

In 2015, we initiated the SOLACE research project to develop strategies to prevent relapse after IMPT progammes. Because there was little research available on this topic (Morley, 2008; Turk \& Rudy, 1991), we started with an 18-month co-design project, in which patients, health care providers, researchers, and designers shared their expertise and collaborated to develop ideas, concepts, and strategies to prevent relapse after successful treatment. This resulted in a prototype paper workbook that contained the two most promising strategies: a valued-based goal-setting procedure, and a method for storing and facilitating retrieval of meaningful treatment experiences. Subsequently, we performed a feasibility study in which the prototype workbook was tested at two different IMPT progammes for 6 months. Overall, patients and health care providers were willing and able to use the workbook and 
regarded the strategies to be in line with the IMPT treatment principles (Elbers et al., 2020). The evaluations also yielded specific suggestions for further improvements, including a preference for a mobile app instead of a paper workbook, along with more interaction between both strategies and a modified goal-setting procedure. In the ensuing research project (i.e. the AGRIPPA project), we used the insights of the feasibility study to improve both strategies and transferred the content of the workbook prototype to an mHealth app. Similar to the initial co-design project, patients, health care providers, designers, and researchers collaborated to optimize usability and intervention components of the app. For example, we explored direct user experience through think-aloud sessions with digital mockups, and we organized cocreation sessions to prioritize app features and to prepare a list of requirements.

\section{Study Objectives}

The present trial has three main objectives. Our first objective is to evaluate the effectiveness of the AGRIPPA intervention (i.e. enhanced treatment condition) on pain disability for patients with chronic musculoskeletal pain who participate in an IMPT progamme compared to a usual care control group with a follow-up period of 12 months. Our second objective is to determine the cost-effectiveness of the AGRIPPA intervention relative to usual care. Our third objective is to explore the level of engagement and perceived usability of patients that use the app. We have formulated three hypotheses: (1) the maintenance of improvement in pain-related disability over time after IMPT will be more favorable for the enhanced treatment condition compared to usual care; (2) the effect of the app on pain disability will translate to less health care utilization and less societal costs (e.g., less absenteeism), leading to a cost-effective intervention compared to treatment as usual; and (3) for participants in the enhanced treatment condition, the perceived usability, frequency of use, duration of use, and reported adherence to the AGRIPPA app will be positively associated with a favorable change over time of pain-related disability.

\section{METHODS}

\section{Design}

We will perform a randomized controlled multicenter superiority trial with two parallel groups in the Netherlands. Both groups will receive standard IMPT, but the experimental group will be provided with the AGRIPPA app that they can access both during and after the treatment progamme. The nature of the intervention does not allow for masking the condition for health care providers or patients. The allocation ratio will be 1:1. 


\section{Ethical Approval}

The study activities have been reviewed and approved by the Medical Research Ethics Committee Utrecht (19/406/D). Protocol modifications that will result in significant changes of study objectives, design, or procedures will require approval by the AGRIPPA steering committee and the Medical Research Ethics Committee.

The trial will be coordinated by a senior researcher of the Lifestyle \& Health Research Group of the University of Applied Sciences Utrecht. The AGRIPPA consortium that consists of all project partners (including the participating treatment centers) meets twice per year to discuss overall progress and topics such as dissemination. The AGRIPPA steering committee consists of researchers who meet four times per year to oversee the quality of the research and to decide on any substantial amendments to the initial project idea.

\section{Study Setting}

Six treatment facilities that provide IMPT progammes participate in this study (2 hospital units and 4 rehabilitation clinics). All locations provide an interdisciplinary biopsychosocial-oriented treatment progamme to patients within the region, leading to a mixed rural and urban population throughout the Netherlands (i.e. Arnhem, Eindhoven, Hoensbroek, Roermond, Maastricht, and Wijk aan Zee).

\section{Eligibility Criteria}

Patients. All patients who participate in one of the treatment progammes will be eligible to participate in this study. To be admitted to one of the treatment progammes, patients must be over 18 years of age and referred by a general practitioner or medical specialist for IMPT. Furthermore, patients must have received a diagnosis of chronic musculoskeletal pain (i.e. pain localized in the muscles, tendons, bones, and joints) that lasts or recurs for more than 3 months, and significantly interferes with physical, psychological, and social functioning. Patients have to consent to a biopsychosocial form of treatment and to participate actively throughout the treatment progamme. Patients with dominant psychiatric comorbidities (e.g., severe depression) and pending legal procedures that are thought to interfere with rehabilitative success will not be eligible for treatment. In all participating treatment facilities, this standard screening procedure is performed by a physician in rehabilitation medicine.

Health Care Providers. To qualify for participation, treatment teams will be required to attend a workshop where they will receive instruction on how to adhere to the study protocol and how to use the app. In addition, health care providers will be instructed to document notable or unexpected events and to participate in a focus group after the study 
period. Each treatment location is also required to provide a research assistant who is not involved with the treatment progamme. This research assistant will be responsible for performing the treatment allocation procedure.

\section{Recruitment}

All patients who will start an IMPT progamme during the study inclusion period will be contacted by their treating physician in rehabilitation medicine and will receive a patient information letter. The patient will then have 1 to 2 weeks to consider participation and to ask any additional questions before signing the informed consent form. Instruction of the app will follow when a signed informed consent form has been provided to the research assistant. An independent research counselor will be available to all participating patients for any questions and general support during the study. Her email address will be provided via the patient information letter.

\section{Randomization}

In participating treatment centers, treatment progammes either consist of group sessions, individual sessions, or their combination. To prevent contamination, we will perform group randomization when treatment is predominantly provided in groups. When treatment is provided in individual sessions, we will randomize each patient individually using a simple randomization procedure. The randomization will be performed using random allocation cards based on computer-generated random numbers. The randomization procedure will be executed by a research assistant who is not providing treatment, and will be concealed from health care providers and patients using a set of sequentially numbered opaque and sealed envelopes. The group randomization procedure will be performed in a similar manner for each group that contains at least one participating patient.

\section{Interventions}

Standard Treatment. Although there is variation in the content of each treatment progamme, they all adopt a similar biopsychosocial perspective on pain management (Dutch Pain Society \& Samenwerkingsverband Pijnpatiënten naar één stem, 2017; Gatchel et al., 2014). Furthermore, each progamme includes (cognitive) behavioural treatment modalities (e.g., graded activity, exposure in vivo, acceptance and commitment therapy) as well as pain neuroscience education, active patient involvement, and structured team meetings to coordinate treatment and evaluate each patient's progress. All progammes are supervised by a physician in rehabilitation medicine who is responsible for patient screening, assessment, and monitoring of overall progress. The treatment team always includes a psychologist and a physical therapist or occupational therapist. At each location, patients start with pain education, where a biopsychosocial orientation toward pain will 
be explained. Patients also receive a manual that provides general information about the treatment progamme. All therapies are delivered at the location of the treatment center, except for optional workplace visits. Relapse prevention is part of regular treatment and is addressed during group treatment, individual counseling, or through specific assignments (e.g., composing a relapse prevention plan).

Enhanced Treatment. In addition to the regular treatment condition, patients in the enhanced treatment condition will be provided access to the AGRIPPA app that is available on the Android and iOS operating systems for mobile devices. The app consists of three components: two behavioural strategies and an education module. "Insight Cards" and "Value-Based Goals" are specific behaviour regulation strategies that have been derived from the SOLACE study and aim to prevent relapse. In addition, an information and education module has been developed in response to patient and health care provider preferences that were expressed during the app design phase.

Component 1: Insight Cards. Patients can use "Insight Cards" to document any personally meaningful experience, thought, or idea that relates to their treatment or corresponding personal development. The main aim is that these "insights" remain accessible after treatment and thereby support the transfer of key treatment principles to each patient's personal context. When capturing an insight, patients first type a title and a short description. Subsequently, they have the possibility to assign a corresponding value and a related picture to the insight (see Figure 1). When patients routinely save their experiences in the app during their treatment period, this will result in a chronologic overview of key experiences over time. During treatment, health care providers will be able to discuss this content with patients to check whether the treatment was received as intended. For patients, the app provides a means to reflect on important experiences during and after treatment. The app also enables patients to mark specific experiences as "favorite" and to share their Insight Cards via email or WhatsApp with their relevant others. After treatment, patients have continuous access to their personal collection of Insight Cards to recover specific insights, to explain specific insights of their condition or treatment to other people, or to reflect on their experiences. Moreover, they can add new Insight Cards to their collection after treatment completion if they have experienced relevant events or insights.

Component 2: Value-Based Goals. The Value-Based Goals module facilitates the formulation of meaningful goals (e.g., going for a hike with friends on Saturday morning to be sociable) and subsequent action planning of each consecutive step toward this goal. The procedure is divided into four steps. First, patients formulate their overall goal. Second, patients reflect on desirability, self-efficacy, estimated time to achievement, and social support concerning this goal. Third, patients formulate a corresponding higher-order value (e.g., values such 
as loyalty, friendship, or adventurousness in relation to a goal of going hiking with old classmates), and, optionally, a reward once the goal has been achieved. In the final part, patients can plan multiple "steps," comprising single or recurrent activities that need to be performed to achieve the corresponding goal (e.g., buying hiking shoes or planning a weekly training session). For each step, patients plan where and when the specific activity will be performed. They can also add reminders or schedule each step in their mobile calendar. In the final part of the step-planning sequence, patients reflect on potential barriers and formulate coping strategies in anticipation of these barriers (e.g., preparing dinner in advance to avoid missing a training session). If a goal has been achieved, patients can directly create an Insight Card of this particular experience. Figure 1 (middle) shows an overview of steps within a specific goal.
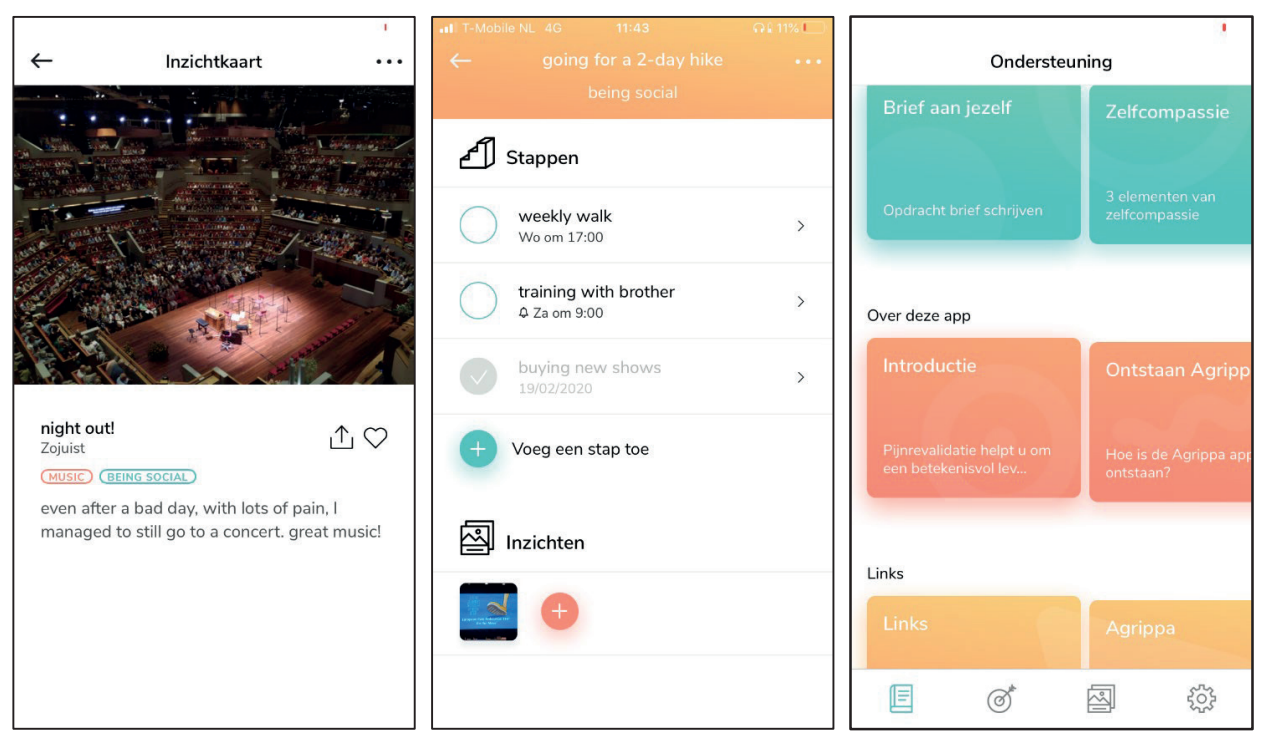

Figure 1. Three Screenshots From the AGRIPPA app.

Notes: Left: example of an insight card, including a related photo, title, two associated values, and description. Middle: Overview of a fictitious goal to go for a hike, including three steps and a related insight. Right: Overview screen of the education and information module. The first row includes treatment-specific exercises, the second row includes general information about the app, and the third row includes links to other support materials.

Component 3: Information and Education. By default, the Information and Education module includes general information about the AGRIPPA project and instructions on how to use both strategies. In addition, each treatment center can add specific content to this section, including information materials, website links, figures, embedded videos, and 
assignments. The main reason for including this module is to create a single environment for all supplementary materials of the treatment progamme. Figure 1 (right) depicts the main screen of this module.

Modifications. During the training session, we recommend health care providers to regularly discuss the app during treatment. However, due to expected variations in digital literacy and other urgent topics, health care providers are allowed to modify the intensity according to their clinical judgement.

Adherence to the Intervention Protocol. The app will be made available to the members of the treatment teams to become accustomed to the content. The training will be provided by two researchers (JP and ES) and include an overview of the purpose and rationale of the study, as well as detailed instructions on how to use the app within the context of the treatment progamme. This includes identifying an appropriate moment within the treatment progamme to introduce the app, determining which member of the team will be responsible for the introduction and encouragement to regularly evaluate the app content, and provide feedback to the participant. During the study, a researcher will have biweekly contact with the treatment team to obtain feedback and discuss progress. Furthermore, two audit sessions will be planned at each location. The researcher will schedule an appointment to discuss overall progress, protocol adherence, and to share examples of good practice with the treatment team. During these sessions, the researcher will also inquire about substantial deviations from the protocol or discontinuation of the app during treatment.

Concomitant Care. During the treatment progamme, patients are not allowed to be treated elsewhere for their chronic pain unless the treatment team decides to refer the patient for a specific reason.

\section{Outcomes}

All six treatment facilities routinely collect outcome data with an electronic survey to monitor their patients (Pollard, 1984). All demographics, baseline measurements, as well as the primary outcome will be obtained by this procedure. Any outcome measures that are not part of the routine assessment will be obtained through an additional electronic survey. Table 1 includes an overview of all outcome domains, measures, and planned analysis methods. The participant flow that is depicted in Figure 2 includes all time points of data collection in this study. A researcher who is not involved with the treatment progamme will monitor all incoming data and will promote study retention using email reminders after 7 days and telephone reminders at 15 and 21 days post measurement. 
Table 1. Planned Outcome Domains, Outcome Measures, and Statistical Methods.

\begin{tabular}{|c|c|c|c|}
\hline Outcome & Hypothesis/research question & Outcome measure & $\begin{array}{l}\text { Method of } \\
\text { analysis }\end{array}$ \\
\hline \multicolumn{4}{|l|}{ Primary } \\
\hline $\begin{array}{l}\text { Self-perceived pain } \\
\text { disability }\end{array}$ & $\begin{array}{l}\text { The development of pain disability over } \\
\text { time after IMPT }{ }^{\text {a }} \text { will be more favorable } \\
\text { for the enhanced treatment condition } \\
\text { compared to usual care. }\end{array}$ & $\mathrm{PDI}^{\mathrm{b}, \mathrm{c}}$ & $\begin{array}{l}\text { Multilevel } \\
\text { analysis }\end{array}$ \\
\hline $\begin{array}{l}\text { Planned subgroup } \\
\text { analysis on patients } \\
\text { that scored } 1 \text { ("very } \\
\text { much better") or } 2 \\
\text { ("much better") on the } \\
\text { GPE }^{\mathrm{d}} \text { scale at } t_{1} \text {. }\end{array}$ & $\begin{array}{l}\text { For patients that experienced } \\
\text { meaningful treatment success, the } \\
\text { development of pain disability over time } \\
\text { after IMPT will be more favorable for the } \\
\text { enhanced treatment condition compared } \\
\text { to usual care. }\end{array}$ & $\begin{array}{l}\text { 1. } \mathrm{PDI}^{\mathrm{c}} \\
\text { 2. } \mathrm{GPE}^{\mathrm{f}}\end{array}$ & $\begin{array}{l}\text { Multilevel } \\
\text { analysis }\end{array}$ \\
\hline \multicolumn{4}{|l|}{ Secondary (quality of life) } \\
\hline Health care costs & $\begin{array}{l}\text { The enhanced treatment condition will } \\
\text { be cost-effective compared with usual } \\
\text { care at } 3 \text { and } 6 \text { months posttreatment. }\end{array}$ & $\begin{array}{l}\text { 1. SF-12 } 2^{\mathrm{f}, \mathrm{g}} \\
\text { 2. } \mathrm{iPCQ}^{\mathrm{f}, \mathrm{h}}\end{array}$ & $\begin{array}{l}\text { Cost- } \\
\text { effectiveness } \\
\text { analysis }\end{array}$ \\
\hline Illness perception & $\begin{array}{l}\text { The development of illness perceptions } \\
\text { over time after IMPT will be more } \\
\text { favorable for the enhanced treatment } \\
\text { condition compared to usual care. }\end{array}$ & IPQ-K DLV ${ }^{c, i}$ & $\begin{array}{l}\text { Multilevel } \\
\text { analysis }\end{array}$ \\
\hline Pain self-efficacy & $\begin{array}{l}\text { The development of pain self-efficacy } \\
\text { over time after IMPT will be more } \\
\text { favorable for the enhanced treatment } \\
\text { condition compared to usual care. }\end{array}$ & PSEQ $^{\mathrm{c}, j}$ & $\begin{array}{l}\text { Multilevel } \\
\text { analysis }\end{array}$ \\
\hline Pain intensity & $\begin{array}{l}\text { The development of outcome measures } \\
\text { over time may be affected by the level of } \\
\text { pain intensity }\end{array}$ & $\mathrm{NRS}^{\mathrm{c}, \mathrm{k}}$ & $\begin{array}{l}\text { Multilevel } \\
\text { analysis }\end{array}$ \\
\hline \multicolumn{4}{|l|}{ Engagement and usability } \\
\hline Overall engagement & $\begin{array}{l}\text { The level of engagement and usability } \\
\text { will be positively associated with a } \\
\text { change in pain disability during follow } \\
\text { up }\left(t_{4}^{1}-t_{1}\right)\end{array}$ & $\begin{array}{l}\text { 1. System usability scale } \\
\text { 2. System usage data of } \\
\text { AGRIPPA app (see below) }\end{array}$ & $\begin{array}{l}\text { Multiple linear } \\
\text { regression }\end{array}$ \\
\hline $\begin{array}{l}\text { Frequency of } \\
\text { engagement }\end{array}$ & $\begin{array}{l}\text { How does the frequency of engagement } \\
\text { vary over time after treatment? }\end{array}$ & $\begin{array}{l}\text { Average number of logins } \\
\text { per week }\end{array}$ & $\begin{array}{l}\text { Descriptive } \\
\text { statistics }\end{array}$ \\
\hline Depth of engagement & $\begin{array}{l}\text { How does the average number of } \\
\text { features accessed per login vary after } \\
\text { treatment? }\end{array}$ & $\begin{array}{l}\text { Average number of } \\
\text { features accessed per } \\
\text { log-in }\end{array}$ & $\begin{array}{l}\text { Descriptive } \\
\text { statistics }\end{array}$ \\
\hline $\begin{array}{l}\text { Duration of } \\
\text { engagement }\end{array}$ & $\begin{array}{l}\text { How do the average minutes spent at } \\
\text { each login as well as the total time } \\
\text { spent with the app per week vary after } \\
\text { treatment? }\end{array}$ & $\begin{array}{l}\text { 1. Average minutes spent } \\
\text { at each login. } \\
2 \text {. Total time spent with } \\
\text { the app }\end{array}$ & $\begin{array}{l}\text { Descriptive } \\
\text { statistics }\end{array}$ \\
\hline
\end{tabular}


Table 1. Continued.

\begin{tabular}{llll}
\hline Outcome & Hypothesis/research question & Outcome measure & $\begin{array}{l}\text { Method of } \\
\text { analysis }\end{array}$ \\
\hline $\begin{array}{l}\text { Active engagement } \\
\text { with Insight Cards }\end{array}$ & $\begin{array}{l}\text { To what extent will the number of } \\
\text { created Insight Cards increase or } \\
\text { decrease after treatment? }\end{array}$ & $\begin{array}{l}\text { Number of Insight Cards } \\
\text { created }\end{array}$ & $\begin{array}{l}\text { Descriptive } \\
\text { statistics }\end{array}$ \\
$\begin{array}{l}\text { Active engagement } \\
\text { with } \mathrm{VBG}^{\mathrm{m}}\end{array}$ & $\begin{array}{l}\text { To what extent will the number of } \\
\text { created VBG cards increase or decrease } \\
\text { after treatment? }\end{array}$ & $\begin{array}{l}\text { 1. Number of VBGs } \\
\text { created. } \\
2 . \text { Number of steps } \\
\text { created }\end{array}$ & $\begin{array}{l}\text { Descriptive } \\
\text { statistics }\end{array}$ \\
\hline
\end{tabular}

Notes: aMPT: interdisciplinary multimodal pain therapy. ${ }^{\mathrm{P} P D I}$ : Pain Disability Index. ${ }^{\mathrm{c} O u t c o m e}$ measure is part of routine care. ${ }^{\mathrm{d} G P E}$ :

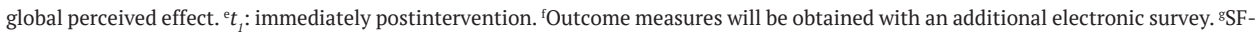
12: 12-item short-form health survey. hiPCQ: iMTA Productivity Cost Questionnaire. iPQ-K DLV: Dutch language version of the Illness Perception Questionnaire. ${ }^{\mathrm{P} S E Q}$ : Pain Self-Efficacy Questionnaire. ${ }^{\mathrm{k}} \mathrm{NRS}$ : numeric rating scale. ${ }^{1} t_{4}: 12$ months postintervention. ${ }^{\mathrm{m} V B G:}$ Value-Based Goals.

Primary Outcome. Self-perceived pain disability will be measured with the Pain Disability Index (PDI) (Soer et al., 2012). This questionnaire consists of 7 items ranging from 1 (no disability) to 10 (maximum disability). Each item relates to the self-reported disability in the context of family/home, recreation, social, occupation, sexual, self-care, and life support. The PDI score (0-70) is obtained by summing all individual items. The PDI score is evaluated as responsive, with a change of 8.5 to 9.5 points considered to be clinically important (Soer et al., 2012).

\section{Secondary Outcomes.}

iMTA Medical Cost Questionnaire. The iMTA Medical Cost Questionnaire (iMCQ) measures all health care costs that have been made during a specific period (Bouwmans et al., 2018a). The questionnaire contains 18 items that refer to 11 primary care components (e.g., medication use, general practitioner visits) and 5 secondary care components (e.g., hospital visits). Two optional questions will be added that relate to informal care by relatives and health care-related travel expenses. Furthermore, we will add two questions from the iMTA Productivity Cost Questionnaire (iPCQ) to acquire an indication of the productivity losses, as the number of hours work lost due to the disease (Bouwmans et al., 2018b; Bouwmans et al., 2015). 


\begin{tabular}{|c|c|c|c|c|c|c|}
\hline & \multicolumn{6}{|c|}{ STUDY PERIOD } \\
\hline \multirow[b]{2}{*}{ TIME POINT } & \multirow{2}{*}{$\begin{array}{c}\text { Enrolment } \\
-t_{1}\end{array}$} & \multirow{2}{*}{$\begin{array}{c}\text { Allocation } \\
0\end{array}$} & \multicolumn{3}{|c|}{ Post-allocation } & \multirow[b]{2}{*}{$t_{4}$} \\
\hline & & & $t_{1}$ & $t_{2}$ & $t_{3}$ & \\
\hline \multicolumn{7}{|l|}{ ENROLMENT: } \\
\hline \multirow{2}{*}{$\begin{array}{r}\text { Eligibility screen } \\
\text { Informed } \\
\text { consent }\end{array}$} & $\mathbf{x}$ & & & & & \\
\hline & $\mathbf{x}$ & & & & & \\
\hline Allocation & & $\mathbf{x}$ & & & & \\
\hline \multicolumn{7}{|l|}{ INTERVENTIONS: } \\
\hline \multicolumn{7}{|l|}{$\begin{array}{c}\text { Standard } \\
\text { treatment }\end{array}$} \\
\hline \multicolumn{7}{|l|}{$\begin{array}{c}\text { Enhanced } \\
\text { treatment }\end{array}$} \\
\hline \multicolumn{7}{|l|}{ ASSESSMENTS: } \\
\hline $\begin{array}{r}\text { Patient } \\
\text { characteristics }\end{array}$ & $\mathbf{x}$ & & & & & \\
\hline PDI & $\mathbf{X}$ & & $\mathbf{x}$ & $\mathbf{x}$ & $\mathbf{x}$ & $\mathbf{x}$ \\
\hline$I P Q-K(D L V)$ & $\mathbf{x}$ & & $\mathbf{x}$ & $\mathbf{x}$ & $\mathbf{x}$ & $\mathbf{x}$ \\
\hline$P S E Q$ & $\mathbf{X}$ & & $\mathbf{x}$ & $\mathbf{x}$ & $\mathbf{x}$ & $x$ \\
\hline $\begin{array}{r}\text { NRS Pain } \\
\text { intensity }\end{array}$ & $\mathbf{X}$ & & $\mathbf{x}$ & $\mathbf{x}$ & $x$ & $\mathbf{x}$ \\
\hline SF12 & & & & $\mathbf{x}$ & $\mathbf{x}$ & \\
\hline GPE & & & $\mathbf{X}$ & $\mathbf{x}$ & $\mathbf{x}$ & $\mathbf{x}$ \\
\hline \multicolumn{7}{|l|}{$\begin{array}{r}\text { System usage } \\
\text { data }\end{array}$} \\
\hline $\begin{array}{r}\text { System Usability } \\
\text { Scale }\end{array}$ & & & $x$ & & & \\
\hline $\begin{array}{r}\text { Cost- } \\
\text { effectiveness }^{*} \\
\text { (iMCQ) }\end{array}$ & & & & $x$ & $\mathbf{x}$ & \\
\hline
\end{tabular}

Figure 2. Schedule of Enrolment, Interventions, and Assessments.

Notes: Figure Adopted from SPIRIT (2013). -t1: prior to treatment; $\mathrm{t} 1$ : posttreatment; $\mathrm{t} 2: 3$ months posttreatment; $\mathrm{t} 3: 6$ months posttreatment; t4: 12 months posttreatment; PDI: pain disability index; IPQ-K (DLV): Dutch language version of the Illness Perception Questionnaire; PSEQ: Pain Self-Efficacy Questionnaire; NRS: numeric rating scale; SF12: 12-item short-form health survey; GPE: global perceived effect. 
Twelve-Item Short Form Health Survey. The 12-item short-form health survey (SF-12) contains 12 multiple choice items that cover 8 health status domains. Raw item scores are combined and transformed into two summary scores: a physical and a mental component score (Cunillera et al., 2010). Higher scores reflect better mental or physical functioning. The SF-12 is considered valid and reliable and has been previously used in patients with chronic pain (Aaronson et al., 1998; Hoffman \& Dukes, 2008; Luo et al., 2003; Picavet \& Hoeymans, 2004).

Pain Self-Efficacy Questionnaire. The Pain Self-Efficacy Questionnaire (PSEQ) assesses self-efficacy beliefs regarding daily life goals in the context of chronic pain (Nicholas, 1989; Nicholas, 2007). The questionnaire includes 10 items that ask a patient to indicate the degree of confidence to perform specific activities (e.g., socializing with friends) despite the pain. Responses are obtained using a Likert scale, ranging from 0 (not confident at all) to 6 (completely confident). The sum of individual scores indicates the total pain self-efficacy. A study on the psychometric properties in a Dutch population of patients with chronic pain demonstrated that the PSEQ is an internally consistent unidimensional instrument (Van Der Maas et al., 2012).

Brief Illness Perceptions Questionnaire Dutch Language Version. The Dutch language version of the Illness Perception Questionnaire (IPQ-K [DLV]) measures how patients evaluate their current health condition with respect to 8 areas of cognitive perception (Leysen et al., 2015). The questionnaire includes 9 items, with 8 items covering a different cognitive area (e.g., controllability) with a Likert-scale response option, ranging from 0 (absolutely no control) to 10 (extreme amount of control). The last item requires the patient to list the 3 most important causes for their current condition. A systematic review on the clinimetric properties of the IPQ-K (DLV) concluded that the questionnaire is appropriate to explore the illness beliefs of various patient groups, including acceptable test-retest reliability. However, the smallest detectable change of 42 (on a maximum of 80 points) implies that the use of an IPQ sum score to detect individual changes is not recommended (Leysen et al., 2015). Therefore, we will not use the sum score but instead evaluate each item separately (de Raaij et al., 2012).

Pain Intensity. We will measure current pain intensity on a numerical rating scale ranging from 0 (no pain) to 10 (worst pain imaginable).

Global Perceived Effect. The global perceived effect (GPE) evaluates to what extent the patient's current condition has improved or worsened compared to the period prior to the treatment progamme. Patients respond with a 5-point Likert scale, ranging from 
1 (very much improved) to 5 (very much worsened). The psychometric properties of this questionnaire have been tested in the context of various musculoskeletal disorders and are considered adequate (Kamper et al., 2010).

System Usability Scale. The system usability scale is a 10-item questionnaire that is frequently used for evaluation of the perceived usability of software apps (Brooke, 1996; Lewis, 2018). Each item is scored on a range from strongly disagree (1) to strongly agree (5). The total usability score is expressed on a 0-100 scale with higher scores indicating more usability.

System Usage Data. To obtain insight into the frequency, intensity, and duration of engagement, we will obtain the following system usage data for each time point: average number of logins per week, average number of features accessed per login, average minutes spent with the app at each login, total time spent with the app per week, number of Insight Cards created, number of value-based goals created, and number of steps created within the Value-Based Goals module.

Intervention Reporting. We will ask each center to provide a detailed overview of their intervention according to the Template for Intervention Description and Replication checklist (Hoffmann et al., 2014). This checklist aims to provide a set of items to describe an intervention for enhancing understanding and replication. Although the interdisciplinary interventions are not the main focus of this study, we will use this intervention to provide an indication of between-center heterogeneity and to assess to what extent the interventions will be modified during the study (item 10).

\section{Data Management}

All study data will be obtained via three electronic sources. The questionnaires will either be collected through routine monitoring procedures within the treatment centers or via additional electronic surveys. System usage data will be provided by the app developer. All study data will be stored within the firewall of the University of Applied Sciences Utrecht (UAS) in a folder on a network drive that is protected by permission rights and will only be available to researchers that are assigned by the project team to analyse the data. Data will be automatically backed up (daily) by the UAS Utrecht. To protect the identity of individual participants, we will perform the following procedures for personally identifiable information. During the data collection phase, we will pseudonymize all incoming data. In the main dataset, we will replace identity data with a unique number (i.e. identifier). Date of birth will be transformed to age in years and address information will not be included in the dataset, except for province and place of residence (rural/urban). To add additional measurements to the dataset and to delete data upon participant request, we will create a 
correspondence table that contains both the identifier and patient personal information. This table will be stored in a separate folder that only the principal investigator (JP) and the head of the Lifestyle \& Health Research Group (HW) can access. Any unforeseen data collection issues that may threaten to reveal an individual's identity will be solved according to the recommendations of Tessier and Bonnemains (2019).

\section{Harm}

We do not expect any serious adverse events as a result of using the app. However, we will monitor any negative consequence that results from usage. During the training of the treatment team, we will instruct the health care providers to report negative experiences of the app (e.g. frustration due to low digital literacy skills). During biweekly contact with treatment teams, the researcher will also actively ask for any adverse event. In the unlikely event of harm, patients can appeal to the liability insurance of the sponsor that covers any damage to research subjects caused by the study within 4 years after the end of the study.

\section{Data Analysis}

During all analyses, the treatment condition will be masked to the researchers. We will analyse the data according to the intention-to-treat principle and include patients in the analysis regardless of their adherence to the treatment protocol. We will perform no interim analysis. We will collect data at the following time points: $-t_{1}$ (baseline), $t_{1}$ (immediately postintervention), $t_{2}$ (3 months postintervention), $t_{3}$ ( 6 months postintervention), and $t_{4}$ (12 months postintervention). Prior to the main analysis, we will check the randomization by examining the distribution of baseline characteristics between both groups.

Sample Size Calculation. The sample size calculation is based on a 2-factor repeatedmeasures analysis of variance with within-factor time (5 levels) and between-factor treatment (2 levels) conditions on the outcome variable PDI. We have set the $\alpha$ to .05, the power $(1-\beta)$ to .95 , and assumed a moderate treatment effect $(f=0.2)$. To account for the expected dependencies of patients within each of the 5 participating treatment locations, we applied the Donner et al (2004) formula for the variance inflation factor, assuming an intracorrelation coefficient of 0.2 (Dickinson \& Basu, 2005; Twisk et al., 2013). Furthermore, we corrected the analysis for an expected attrition of $20 \%$, which is based on the average attrition of similar studies that used the PDI (Hållstam et al., 2017; Mangels et al., 2009; Volker et al., 2017). Based on these calculations, a minimum sample size of 157 participants, equally divided over 5 treatment locations, will be required.

Primary Analysis: Pain Disability. In our primary analysis, we will test the difference in the development of pain disability over time between patients in the enhanced condition and patients in the treatment condition. To account for the assumed dependence of the 
repeated observations and treatment locations, we will perform a multilevel analysis. In our hierarchical model, time points (level 1) will be nested in patients (level 2) and patients nested in treatment locations (level 3). Our main analysis will include the effects of time, treatment condition, treatment location, and the interaction between time and treatment condition, with a random intercept for patients. In addition, the model will be adjusted for sex, age, pain intensity, and pain duration. In the case of a significant 2-way interaction between time and treatment condition, posthoc contrasts between the treatment conditions at 3, 6, and 12 months will be calculated. We will also perform a subgroup analysis for patients that report a positive treatment effect at $t_{1}$ (i.e. a GPE score of 1 or 2).

\section{Analysis of Secondary Outcomes}

Perceived Usability and App Engagement. Based on the results of the feasibility study and the nature of the behaviour regulation strategies, we expect an engagement pattern in frequency, type, and depth of engagement that differs for each behaviour change strategy and changes over time. Specifically, for Insight Cards, we believe that patients will actively engage with this component during treatment (i.e. creating Insight Cards during use), but shift to more passive engagement (i.e. reading the input, but only creating new content at limited occasions) during follow up. For Value-Based Goals, we expect that the formulation of goals and steps will increase during the final part of treatment, together with a growing emphasis within the treatment progamme on integrating newly learned strategies into daily life routines. After treatment, we anticipate that patients will engage in a reflective (e.g., documenting progress) and active (i.e. formulating new goals) manner with this strategy. In general, we expect a decreasing trend of the number of logins over time, but an increase in the "depth" of use (i.e. the average number of features accessed per login) as well as an increase in the duration of a login. We will calculate descriptive statistics to explore patterns of engagement. Furthermore, to examine the extent to which user engagement and usability are negatively associated with the change of pain disability during follow up, we will perform a multiple regression analysis, with the change score of pain disability $\left(t_{4}\right.$ $-t_{1}$ ) as the outcome variable and engagement and usability measures as predictors. We will adjust for age, sex, pain intensity, and baseline PDI.

Cost Effectiveness. To investigate the efficiency of the intervention, we will perform a cost-effectiveness analysis at 3 and 6 months posttreatment according to the intention-totreat principle. We hypothesize that patients in the enhanced treatment condition will have more quality-adjusted life years (QALYs) relative to the health care expenses compared to the regular treatment condition within the 6-month study period. Expected health gain will be expressed in QALYs and calculated using the procedure of Brazier and colleagues (2004) to estimate the 6-dimensional health state form (SF-6D) using the SF-12 assessment at 3 and 6 months posttreatment (Brazier \& Roberts, 2004; Cunillera et al., 2010). 
Intervention costs will be determined by the standardized cost prices for rehabilitation treatment (Hakkaart-van Roijen et al., 2015). Other health expenses will be obtained using the $\mathrm{iMTQ}$ at 3 and 6 months posttreatment. This questionnaire includes visits to health care providers, prescribed and over-the-counter medication, and alternative health care. We will also calculate the productivity loss due to pain-related absence from work, adopting the gross human capital approach (Pike \& Grosse, 2018). Productivity loss will be obtained with two questions of the iPCQ at 3 and 6 months posttreatment. Total costs are calculated using the Dutch manual for cost analysis in health care research (Hakkaart-van Roijen et al., 2015; Kanters et al., 2017). Following the procedure of Den Hollander and colleagues (Den Hollander et al., 2018), a standardized cost price will be used for each hour of productivity loss. Total costs and total health gains for each condition will be used to calculate the incremental cost-effectiveness ratio (ICER). Furthermore, we will construct cost-effectiveness acceptability curves based on mean costs and using incremental costs and incremental effects, employing nonparametric bootstrapping with 5000 replications. This will result in a scatter plot over four quadrants, where each quadrant indicates a different implication for economic evaluation (i.e. a combination of positive or negative costs and effects) (Fenwick \& Byford, 2005).

According to the National Institute for Health Care and Excellence guidelines, all intervention costs in cost-effectiveness analyses should relate to health care or social services funding (NICE, 2013; Li et al., 2018). This excludes the development costs of the AGRIPPA app because this project has been funded with research grants. However, to account for future development and maintenance costs, we will perform a sensitivity analysis and explore various cost scenarios. We will calculate multiple ICERs, each with a different cost input value that corresponds to a possible future pricing scenario (e.g., subscription, pay to download).

Missing Data. Following recommendations of Twisk and colleagues (Twisk et al., 2013), we will perform the multilevel analysis on incomplete data, rather than using multiple imputation procedures. However, we will use the R MICE package to search for patterns of missing data across the included variables and to perform $t$ tests to explore the relationships between the amount of missingness of each variable and all other variables (Tabachnik \& Fidell, 2013).

\section{Participant Timeline}

Patient eligibility screening, informed consent, and treatment allocation procedures, as well as the baseline assessment, will be completed prior to the start of the treatment progamme. Patients in the enhanced treatment condition will receive instruction on how to download and use the app, and both strategies will be explained by a member of the treatment staff. 
To match the existing treatment content and procedures, the moment that the app will be introduced to patients can vary between locations. During the treatment phase, the app is considered a supplement to the main treatment activities and patients are free to decide how and when to use the app. Health care providers will be instructed to encourage and facilitate using the strategies when they expect it will reinforce the treatment process. However, both strategies can largely be used independently of any treatment activity and are expected to minimally affect direct treatment time. A member of the research team will be available to the treatment staff throughout the experimental phase for additional questions, support, and discussions regarding optimal use. Following treatment, there will be no additional monitoring in the enhanced treatment condition. Patients will continue to be able to use the app at their discretion. Posttreatment data will be obtained directly posttreatment (t1), and at 3 months (t2), 6 months (t3), and 12 months (t4) posttreatment.

\section{RESULTS}

The trial has been registered in the Netherlands Trial Register under the identifier NL8076. The study is ongoing. The patient inclusion period started in October 2019 and is expected to end in November 2020. As of March 20, 2020, we have recruited 88 patients. Results are expected to be released in the final quarter of 2021. In the last meeting of 2020, the steering committee will initiate the formation of writing teams that will be responsible for the final trial report.

\section{DISCUSSION}

\section{Study Goals}

This study will evaluate the AGRIPPA app in the context of interdisciplinary multimodal pain treatment progammes. Specifically, we will investigate the effect of app use on longterm pain disability and efficiency by means of a cost-effectiveness analysis. To discover how patients interact with the app, we will also explore usability and engagement and test the impact of these variables on pain disability. Together, these analyses will help to demonstrate to what extent the AGRIPPA app contributes to preventing relapse in painrelated disability.

In contrast to prevailing intervention development guidelines, the AGRIPPA development project adopted a co-design approach and started with collecting qualitative data from end users (e.g., patients and health care providers) rather than with formulating a theoretical framework. Although co-design is increasingly acknowledged in the health care domain 
as a method to integrate stakeholder input into the intervention design, more robust evaluations of co-design-based interventions are required to determine its additional value to existing development practices (Donetto et al., 2015; Robert \& Macdonald, 2017). A similar point can be made for the evaluation of mHealth apps. A recent systematic review revealed that health care apps to promote self-management in chronic conditions have seldom been evaluated by randomized controlled trials over a prolonged study period (Byambasuren et al., 2018). This study will help understand if an mHealth app that has been developed by co-design methods not only contributes to an acceptable and user-friendly intervention but also leads to maintenance of treatment gains for patients with chronic pain.

\section{Strengths and Limitations}

Because IMPT progammes often substantially vary in dose and content, the inclusion of multiple treatment centers will positively influence generalization. Furthermore, this study builds on a feasibility study where evaluations related to form, content, and integration within treatment progammes have been incorporated into the current app and study procedures.

The exploratory analysis of engagement variables in this study is expected to provide preliminary insight into patient adherence to the behaviour regulation strategies within the app. According to Sieverink and colleagues (Sieverink et al., 2017), insight into adherence to mHealth apps can be acquired by combining usage behaviour data with a description of intended use and a well-substantiated justification for this intended use. Although this may be difficult to quantify as the intended usage of the app depends on fluctuations of patients' functional status in the posttreatment phase, the comparison of system usage data with our expectations regarding the use of the strategies will at least provide an indication of adherence to the app. Possible follow-up studies that include qualitative evaluations of patient input may lead to a more sustained insight into adherence.

This study includes several challenges and compromises that can potentially bias the outcomes. First, including patients with both treatment conditions within one center increases the risk of contamination. Second, health care providers have a large influence on participant engagement. Our feasibility study indicated that healthcare provider involvement varied greatly between patients, and that patients with limited health care provider feedback did not always use the intervention as intended (Elbers et al., 2020). By scheduling regular contact moments to discuss progress, we aim to minimize the impact of this potential threat. Third, the limited project duration and funding resulted in a maximum follow-up period of 12 months. Although this first year may be crucial for integrating the newly learned management strategies into a daily life routine, the effect of the AGRIPPA app 
on late-onset occurrences of relapse will not be monitored in this study. Limited funding also prevented the development of an active (mHealth) control condition. Although opting for a treatment-as-usual condition as a comparator is a widely used method, this does not control for the potential placebo effect of receiving an mHealth app. Fourth, to minimize the impact for patients to participate in this study, we have selected a limited number of outcome measures and measurement time points in addition to routine assessments. This may result in an increased recall bias or limited insight in potential factors that could explain a possible effect of the app. Finally, patients will require sufficient digital literacy skills to effectively use the app, which may lead to self-selection during the recruitment phase. The selection bias may threaten generalization.

\section{Implications for Practice}

Maintaining behaviour change is notoriously difficult to achieve and every small step toward decreasing relapse or understanding the specific mechanisms by which relapse occurs or is prevented will be important to the field of pain rehabilitation. Implementation of this intervention in treatment progammes may also positively empower patients to take a more proactive role in their treatment progamme and increase sharing their experiences, thoughts, and beliefs with health care providers and significant others. This may not only lead to a better patient-health care provider relationship and improved mutual understanding but is also expected to positively influence adherence to newly learned pain management strategies (Butow \& Sharpe, 2013).

\section{Acknowledgments}

This project is funded by a SIA-RAAK publiek grant (RAAK.PUB05.002).

\section{Author Contributions}

SE prepared the manuscript; JP, HW, AK, ES, and RS provided feedback; JP and HW are grant holders; JP obtained medical ethical approval, is the principal investigator, and is responsible for the day-to-day management of the "AGRIPPA" research project; HW, AK, and RS are steering committee members of AGRIPPA and monitor overall progress; ES is the contact person for the treatment locations.

\section{Conflicts of Interest}

None declared.

\section{Abbreviations}

GPE: global perceived effort; ICER: incremental cost-effectiveness ratio; IMPT: interdisciplinary multimodal pain therapy; iMTQ: iMTA Medical Cost Questionnaire; iPCQ: 
iMTA Productivity Cost Questionnaire; IPQ-K (DLV): Illness Perception QuestionnaireDutch language version; mHealth: mobile health; PDI: Pain Disability Index; PSEQ: Pain Self-Efficacy Questionnaire; QALYs: quality-adjusted life years; SF-6D: 6-dimensional health state form; SF-12: 12-item short-form health survey; UAS: University of Applied Sciences Utrecht. 


\section{REFERENCES}

Aaronson, N. K., Muller, M., Cohen, P. D. A., Essink-Bot, M. L., Fekkes, M., Sanderman, R., Sprangers, M. A. G., Te Velde, A., \& Verrips, E. (1998). Translation, validation, and norming of the Dutch language version of the SF-36 Health Survey in community and chronic disease populations. J Clin Epidem, 51(11), 1055-1068. https://doi. org/10.1016/S0895-4356(98)00097-3

Anglada-Martinez, H., Riu-Viladoms, G., Martin-Conde, M., Rovira-Illamola, M., Sotoca-Momblona, J. M., \& CodinaJane, C. (2015). Does mHealth increase adherence to medication? Results of a systematic review. Int J Clin Pract, 69(1), 9-32. https://doi.org/10.1111/ijcp.12582

Banerjee, S., \& Argáez, C. (2017). Multidisciplinary Treatment Programs for Patients with Chronic Non-Malignant Pain: A Review of Clinical Effectiveness, Cost-Effectiveness, and Guidelines. In Multidisciplinary Treatment Programs for Patients with Chronic Non-Malignant Pain: A Review of Clinical Effectiveness, Cost-Effectiveness, and Guidelines. Canadian Agency for Drugs and Technologies in Health. http:/www.ncbi.nlm.nih.gov/ pubmed/30188646

Bouwmans, C., Hakkaart-van Roijen, L., Koopmanschap, M., Severens, H., \& Brouwer, W. (2018a). Manual iMTA Medical Cost Questionnaire (iMCQ). https://www.imta.nl/questionnaires/

Bouwmans, C., Hakkaart-van Roijen, L., Koopmanschap, M., Severens, H., \& Brouwer, W. (2018b). Manual iMTA Productivity Cost Questionnaire (IPCQ). https://www.imta.nl/questionnaires/

Bouwmans, C., Krol, M., Severens, H., Koopmanschap, M., Brouwer, W., \& van Roijen, L. H. (2015). The iMTA Productivity Cost Questionnaire: A Standardized Instrument for Measuring and Valuing Health-Related Productivity Losses. Value Health, 18(6), 753-758. https://doi.org/10.1016/j.jval.2015.05.009

Brazier, J. E., \& Roberts, J. (2004). The estimation of a preference-based measure of health from the SF-12. Med Care, 42(9), 851-859. https://doi.org/10.1097/01.mlr.0000135827.18610.0d

Breivik, H., Collett, B., Ventafridda, V., Cohen, R., \& Gallacher, D. (2006). Survey of chronic pain in Europe: Prevalence, impact on daily life, and treatment. Eur J Pain, 10(4), 287. https://doi.org/10.1016/j.ejpain.2005.06.009

Brooke, J. (1996). SUS - A quick and dirty usability scale. In P. Jordan, B. Thomas, I. McClelland, \& B. Weerdmeester (Eds.), Usability evaluation in industry. London: Taylor \& Francis.

Butow, P., \& Sharpe, L. (2013). The impact of communication on adherence in pain management. Pain, 154(SUPPL. 1). https://doi.org/10.1016/j.pain.2013.07.048

Byambasuren, O., Sanders, S., Beller, E., \& Glasziou, P. (2018). Prescribable mHealth apps identified from an overview of systematic reviews. Npj Digit Med, 1(1). https://doi.org/10.1038/s41746-018-0021-9

Coursaris, C. K., \& Kim, D. J. (2011). A Meta-Analytical Review of Empirical Mobile Usability Studies. J Usabil Stud, 6(3), 117-171.

Cunillera, O., Tresserras, R., Rajmil, L., Vilagut, G., Brugulat, P., Herdman, M., Mompart, A., Medina, A., Pardo, Y., Alonso, J., Brazier, J., \& Ferrer, M. (2010). Discriminative capacity of the EQ-5D, SF-6D, and SF-12 as measures of health status in population health survey. Qual Life Res, 19(6), 853-864. https://doi.org/10.1007/s11136010-9639-z

de Raaij, E. J., Schröder, C., Maissan, F. J., Pool, J. J., \& Wittink, H. (2012). Cross-cultural adaptation and measurement properties of the Brief Illness Perception Questionnaire-Dutch Language Version. Man Ther, 17(4), 330-335. https://doi.org/10.1016/j.math.2012.03.001

Den Hollander, M., Heijnders, N., De Jong, J. R., Vlaeyen, J. W. S., Smeets, R. J. E. M., \& Goossens, M. E. J. B. (2018). Exposure in vivo versus pain-contingent physical therapy in complex regional pain syndrome type I. Int $J$ Technol Assess Health Care, 34(4), 352-359. https://doi.org/10.1017/S0266462318000429 
Dickinson, L. M., \& Basu, A. (2005). Multilevel modeling and practice-based research. Ann Fam Med, 3(suppl 1), S52-S60. https://doi.org/10.1370/afm.340

Donetto, S., Pierri, P., Tsianakas, V., \& Robert, G. (2015). Experience based co-design and healthcare improvement: Realizing participatory design in the public sector. Design J, 18(2), 227-248. https://doi.org/10.2752/1756306 15X14212498964312

Donner, A., \& Klar, N. (2004). Pitfalls of and Controversies in Cluster Randomization Trials. Am J Pub Health, 94(3), 416-422. https://doi.org/10.2105/AJPH.94.3.416

Dutch Pain Society, \& Samenwerkingsverband Pijnpatiënten naar één stem. (2017). Zorgstandaard Chronische pijn.

Elbers, S., Pool, J., Wittink, H., Köke, A., \& Smeets, R. (2020). Exploring the feasibility of relapse prevention strategies in interdisciplinary multimodal pain therapy programs: qualitative study. JMIR Hum Fact, 7(4), e21545. https:// doi.org/10.2196/21545

Fenwick, E., \& Byford, S. (2005). A guide to cost-effectiveness acceptability curves. Br J Psychiatry, 187, 106-108. https://doi.org/10.1192/bjp.187.2.106

Fleig, L., Pomp, S., Schwarzer, R., \& Lippke, S. (2013). Promoting exercise maintenance: How interventions with booster sessions improve long-term rehabilitation outcomes. Rehabil Psychol, 58(4), 323-333. https://doi. org/10.1037/a0033885

Foreword | Guide to the methods of technology appraisal 2013 | Guidance | NICE. (2013). https://www.nice.org.uk/ process/pmg9/chapter/foreword

Foster, N. E., Anema, J. R., Cherkin, D., Chou, R., Cohen, S. P., Gross, D. P., Ferreira, P. H., Fritz, J. M., Koes, B. W., Peul, W., Turner, J. A., Maher, C. G., Buchbinder, R., Hartvigsen, J., Underwood, M., van Tulder, M., Menezes Costa, L., Croft, P., Ferreira, M., ... Woolf, A. (2018). Prevention and treatment of low back pain: evidence, challenges, and promising directions. Lancet, 391(10137), 2368-2383. https://doi.org/10.1016/S0140-6736(18)30489-6

Gatchel, R. J., McGeary, D. D., McGeary, C. A., \& Lippe, B. (2014). Interdisciplinary chronic pain management. Am Psychol, 69(2), 119-130. https://doi.org/10.1037/a0035514

Hakkaart-van Roijen, L., van der linden, N., Bouwmans, C., Kanters, T., \& Tan, S. (2015). Kostenhandleiding Methodologie van kostenonderzoek en referentieprijzen voor economische evaluaties in de gezondheidszorg.

Hållstam, A., Löfgren, M., Benson, L., Svensén, C., \& Stålnacke, B. M. (2017). Assessment and treatment at a pain clinic: A one-year follow-up of patients with chronic pain. Scan J Pain, 17, 233-242. https://doi.org/10.1016/j. sjpain.2016.08.004

Hoffman, D. L., \& Dukes, E. M. (2008). The health status burden of people with fibromyalgia: A review of studies that assessed health status with the SF-36 or the SF-12. Int J Clin Pract, 62(1), 115-126. https://doi.org/10.1111/ j.1742-1241.2007.01638.x

Hoffmann, T. C., Glasziou, P. P., Boutron, I., Milne, R., Perera, R., Moher, D., Altman, D. G., Barbour, V., Macdonald, H., Johnston, M., Kadoorie, S. E. L., Dixon-Woods, M., McCulloch, P., Wyatt, J. C., Phelan, A. W. C., \& Michie, S. (2014). Better reporting of interventions: Template for intervention description and replication (TIDieR) checklist and guide. BMJ (Online), 348. https://doi.org/10.1136/bmj.g1687

Kamper, S. J., Apeldoorn, A. T., Chiarotto, A., Smeets, R. J. E. M., Ostelo, R. W. J. G., Guzman, J., \& van Tulder, M. W. (2014). Multidisciplinary biopsychosocial rehabilitation for chronic low back pain. Cochrane Database Syst Rev, 9. https://doi.org/10.1002/14651858.CD000963.pub3

Kamper, S. J., Ostelo, R. W. J. G., Knol, D. L., Maher, C. G., de Vet, H. C. W., \& Hancock, M. J. (2010). Global Perceived Effect scales provided reliable assessments of health transition in people with musculoskeletal disorders, but ratings are strongly influenced by current status. J Clin Epidem, 63(7), 760-766. https://doi.org/10.1016/j. jclinepi.2009.09.009 
Kanters, T. A., Bouwmans, C. A. M., Van Der Linden, N., Tan, S. S., \& Hakkaart-van Roijen, L. (2017). Update of the Dutch manual for costing studies in health care. PLOS ONE, 12(11), e0187477. https://doi.org/10.1371/journal. pone.0187477

Kwasnicka, D., Dombrowski, S. U., White, M., \& Sniehotta, F. (2016). Theoretical explanations for maintenance of behaviour change: a systematic review of behaviour theories. Health Psychol Rev, 10(3), 277-296. https://doi.or g/10.1080/17437199.2016.1151372

Lewis, J. R. (2018). The System Usability Scale: Past, Present, and Future. Int J Hum Comput Interact, 34(7), 577-590. https://doi.org/10.1080/10447318.2018.1455307

Leysen, M., Nijs, J., Meeus, M., Paul van Wilgen, C., Struyf, F., Vermandel, A., Kuppens, K., \& Roussel, N. A. (2015). Clinimetric properties of illness perception questionnaire revised (IPQ-R) and brief illness perception questionnaire (Brief IPQ) in patients with musculoskeletal disorders: A systematic review. Man Ther, 20(1), 10-17. https://doi.org/10.1016/j.math.2014.05.001

Li, J., Parrott, S., Sweeting, M., Farmer, A., Ross, J., Dack, C., Pal, K., Yardley, L., Barnard, M., Hudda, M., Alkhaldi, G., \& Murray, E. (2018). Cost-effectiveness of facilitated access to a self-management website, compared to usual care, for patients with type 2 diabetes (help-diabetes): Randomized controlled trial. J Med Int Res, 20(6). https://doi.org/10.2196/jmir.9256

Linton, S. J., \& Shaw, W. S. (2011). Impact of psychological factors in the experience of pain. Phys Ther, 91(5), 700-711. https://doi.org/10.2522/ptj.20100330

Luo, X., George, M. L., Kakouras, I., Edwards, C. L., Pietrobon, R., Richardson, W., \& Hey, L. (2003). Reliability, validity, and responsiveness of the short form 12-item survey (SF-12) in patients with back pain. Spine, 28(15), 17391745. https://doi.org/10.1097/01.BRS.0000083169.58671.96

Mangels, M., Schwarz, S., Worringen, U., Holme, M., \& Rief, W. (2009). Evaluation of a behavioral-medical inpatient rehabilitation treatment including booster sessions: A randomized controlled study. Clin J Pain, 25(5), 356364. https://doi.org/10.1097/AJP.0b013e3181925791

Middleton, K. R., Anton, S. D., \& Perri, M. G. (2013). Long-term adherence to health behavior change. Am J Journal Lifestyle Med, 7(6), 395-404. https://doi.org/10.1177/1559827613488867

Miller, N. H. (2012). Adherence behavior in the prevention and treatment of cardiovascular disease. J of Cardiopulm Rehabil Prev, 32(2), 63-70. https://doi.org/10.1097/HCR.0b013e318235c729

Monticone, M., Ambrosini, E., Rocca, B., Cazzaniga, D., Liquori, V., \& Foti, C. (2016). Group-based task-oriented exercises aimed at managing kinesiophobia improved disability in chronic low back pain. Eur J Pain, 20(4), 541-551. https://doi.org/10.1002/ejp.756

Morley, S. (2008). Relapse prevention: Still neglected after all these years. Pain, 134(3), 239-240. https://doi. org/10.1016/j.pain.2007.12.004

Morley, S., \& Williams, A. (2015). New developments in the psychological management of chronic pain. Can J Psychiatry, 60(4), 168-175). https://doi.org/10.1177/070674371506000403

Morley, S., Williams, A., \& Eccleston, C. (2013). Examining the evidence about psychological treatments for chronic pain: Time for a paradigm shift? Pain, 154(10), 1929-1931). https://doi.org/10.1016/j.pain.2013.05.049

Nicholas, M. K. (1989). Self-efficacy and chronic pain. Presented at: Annual Conference of British Psychological Society, St. Andrews, Schotland

Nicholas, M. K. (2007). The pain self-efficacy questionnaire: Taking pain into account. Eur J Pain, 11(2), $153-163$. https://doi.org/10.1016/j.ejpain.2005.12.008

Perski, O., Blandford, A., Garnett, C., Crane, D., West, R., \& Michie, S. (2018). A self-report measure of engagement with digital behavior change interventions (DBCIs): Development and psychometric evaluation of the " $\mathrm{dBCI}$ Engagement Scale.” Trans Behav Med, 10(1), 267-277. https://doi.org/10.1093/tbm/ibz039 
Persson, E., Lexell, J., Eklund, M., \& Rivano-Fischer, M. (2012). Positive effects of a musculoskeletal pain rehabilitation program regardless of pain duration or diagnosis. $P M \& R, 4(5), 355-366$. https://doi.org/10.1016/j. pmrj.2011.11.007

Pham, Q., Graham, G., Carrion, C., Morita, P. P., Seto, E., Stinson, J. N., \& Cafazzo, J. A. (2019). A library of analytic indicators to evaluate effective engagement with consumer mHealth apps for chronic conditions: Scoping review. JMIR MHealth and UHealth, 7(1). https://doi.org/10.2196/11941

Picavet, H. S. J., \& Hoeymans, N. (2004). Health related quality of life in multiple musculoskeletal diseases: SF-36 and EQ-5D in the DMC3 study. Ann Rheum Diseases, 63(6), 723-729. https://doi.org/10.1136/ard.2003.010769

Pike, J., \& Grosse, S. D. (2018). Friction cost estimates of productivity costs in cost-of-illness studies in comparison with human capital estimates: A review. App Health Econ Health Pol, 16(6), 765-778. https://doi.org/10.1007/ s40258-018-0416-4

Pollard, C. A. (1984). Preliminary validity study of the pain disability index. Percep Mot Skills, 59(3), 974. https://doi. org/10.2466/pms.1984.59.3.974

Rathbone, A. L., \& Prescott, J. (2017). The use of mobile apps and SMS messaging as physical and mental health interventions: Systematic review. J Med Int Res, 19(8). https://doi.org/10.2196/jmir.7740

Robert, G., \& Macdonald, A. S. (2017). Co-design, organisational creativity and quality improvement in the healthcare sector: ‘designerly’ or ‘design-like’? In D. Sangiorgi \& A. Prendville (Eds.), Design for service: Key issues and new directions (pp. 117-130). Bloomsbury Academic.

Schatman, M. (2012). Interdisciplinary chronic pain management: international perspectives. Pain: Clinical Updates, 20(7), 1-5. https://s3.amazonaws.com/rdcms-iasp/files/production/public/Content/ContentFolders/ Publications2/PainClinicalUpdates/Archives/PCU_20-7_web.pdf

Sieverink, F., Kelders, S. M., \& Gemert-Pijnen, V. (2017). Clarifying the concept of adherence to ehealth technology: Systematic review on when usage becomes adherence. In J Med Int Res, 19(12). https://doi.org/10.2196/ jmir.8578

Silvemark, A., Källmén, H., \& Molander, C. (2014). Improved life satisfaction and pain reduction: Follow-up of a 5-week multidisciplinary long-term pain rehabilitation programme. Upsala J Med Sci, 119(3), 278-286. https:// doi.org/10.3109/03009734.2014.908252

Smeets, R. J. E. M., Vlaeyen, J. W. S., Hidding, A., Kester, A. D. M., van der Heijden, G. J. M. G., \& Knottnerus, J. A. (2008) Chronic low back pain: Physical training, graded activity with problem solving training, or both? The oneyear post-treatment results of a randomized controlled trial. Pain, 134(3), 263-276. https://doi.org/10.1016/j. pain.2007.04.021

Soer, R., Reneman, M. F., Vroomen, P. C. A. J., Stegeman, P., \& Coppes, M. H. (2012). Responsiveness and minimal clinically important change of the pain disability index in patients with chronic back pain. Spine, 37(8), 711715. https://doi.org/10.1097/BRS.0b013e31822c8a7a

Tabachnik, B., \& Fidell, L. (2013). Cleaning up your act: Screening data prior to analysis. In Using multivariate statistics (pp. 60-116). Boston: Pearson.

Tavafian, S. S., Jamshidi, A. R., \& Mohammad, K. (2011). Treatment of chronic low back pain: A randomized clinical trial comparing multidisciplinary group-based rehabilitation program and oral drug treatment with oral drug treatment alone. Clin J Pain, 27(9), 811-818. https://doi.org/10.1097/AJP.0b013e31821e7930

Tessier, C., \& Bonnemains, V. (2019). Experiments With Participants: Some Ethical Considerations. In H. Ayaz \& F. Dehais (Eds.), Neuroergonomics: the brain at work in everyday life (pp. 65-70). Elsevier Academic Press.

Treede, R. D., Rief, W., Barke, A., Aziz, Q., Bennett, M. I., Benoliel, R., Cohen, M., Evers, S., Finnerup, N. B., First, M. B., Giamberardino, M. A., Kaasa, S., Kosek, E., Lavand’homme, P., Nicholas, M., Perrot, S., Scholz, J., Schug, S., Smith, B. H., ... Wang, S. J. (2015). A classification of chronic pain for ICD-11. Pain, 156(6), 1003-1007). https:// doi.org/10.1097/j.pain.0000000000000160 
Turk, D. C., \& Rudy, T. E. (1991). Neglected topics in the treatment of chronic pain patients - relapse, noncompliance, and adherence enhancement. Pain, 44(1), 5-28). https://doi.org/10.1016/0304-3959(91)90142-K

Twisk, J., De Boer, M., De Vente, W., \& Heymans, M. (2013). Multiple imputation of missing values was not necessary before performing a longitudinal mixed-model analysis. J Clin Epidem, 66(9), 1022-1028. https://doi. org/10.1016/j.jclinepi.2013.03.017

Van Der Maas, L. C. C., De Vet, H. C. W., Köke, A., Bosscher, R. J., \& Peters, M. L. (2012). Psychometric properties of the Pain Self-Efficacy Questionnaire (PSEQ): Validation, prediction, and discrimination quality of the Dutch version. Eur J Psychol Ass, 28(1), 68-75. https://doi.org/10.1027/1015-5759/a000092

Vlaeyen, J. W. S., Morley, S., \& Crombez, G. (2016). The experimental analysis of the interruptive, interfering, and identity-distorting effects of chronic pain. Behav Res Ther, 86, 23-34.https://doi.org/10.1016/j.brat.2016.08.016

Volker, G., van Vree, F., Wolterbeek, R., van Gestel, M., Smeets, R., Köke, A., \& Vlieland, T. V. (2017). Long-Term Outcomes of Multidisciplinary Rehabilitation for Chronic Musculoskeletal Pain. Musculoskeletal Care, 15(1), 59-68. https://doi.org/10.1002/msc. 1141

Vos, T., Flaxman, A. D., Naghavi, M., Lozano, R., Michaud, C., Ezzati, M., Shibuya, K., Salomon, J. A., Abdalla, S., Aboyans, V., Abraham, J., Ackerman, I., Aggarwal, R., Ahn, S. Y., Ali, M. K., Almazroa, M. A., Alvarado, M., Anderson, H. R., Anderson, L. M., ... Murray, C. J. L. (2012). Years lived with disability (YLDs) for 1160 sequelae of 289 diseases and injuries 1990-2010: A systematic analysis for the Global Burden of Disease Study 2010. Lancet, 380(9859), 2163-2196. https://doi.org/10.1016/S0140-6736(12)61729-2

Zapata, B. C., Fernández-Alemán, J. L., Idri, A., \& Toval, A. (2015). Empirical Studies on Usability of mHealth Apps: A Systematic Literature Review. J Med Sys, 39(2), 1-19. https://doi.org/10.1007/s10916-014-0182-2 


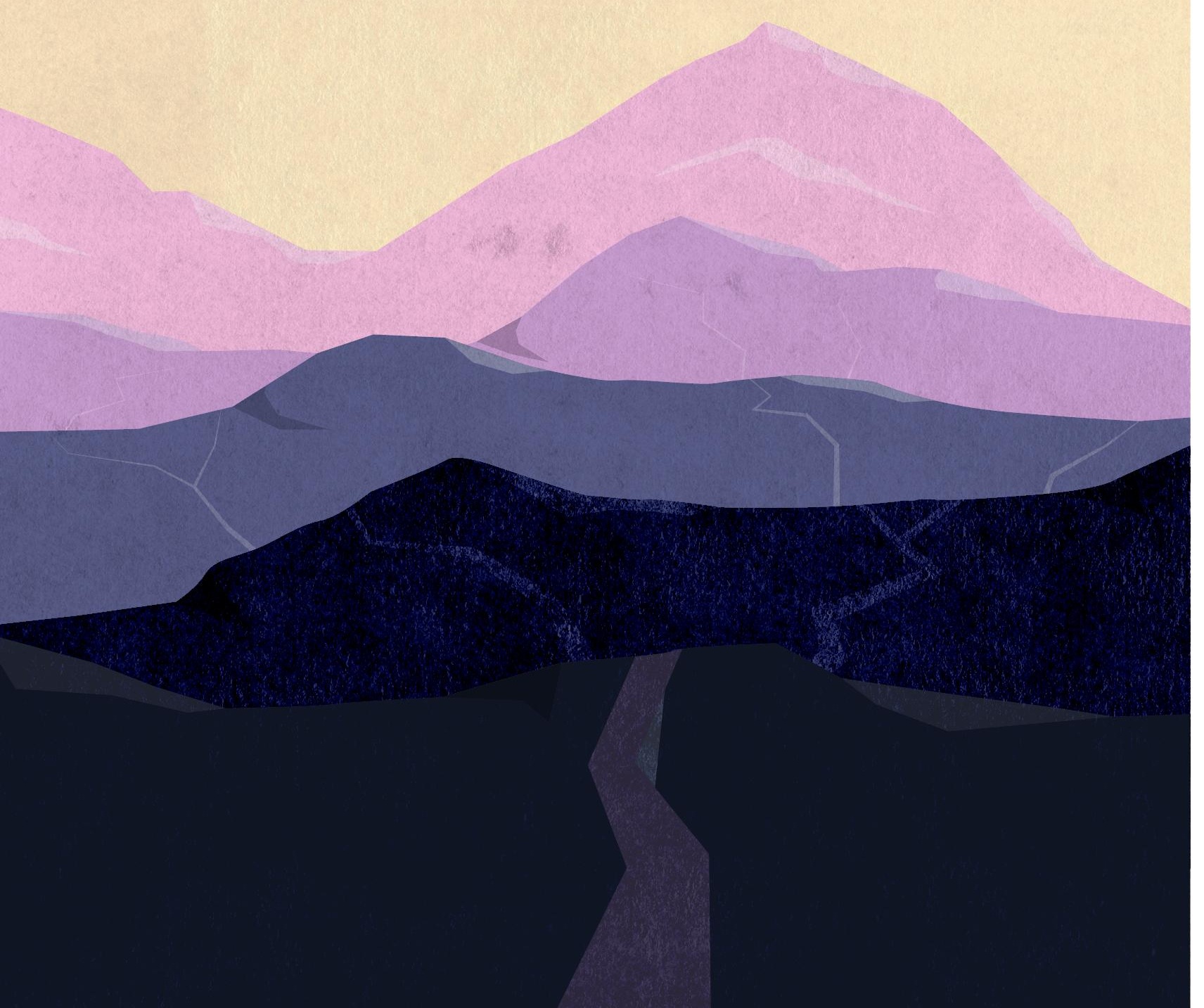




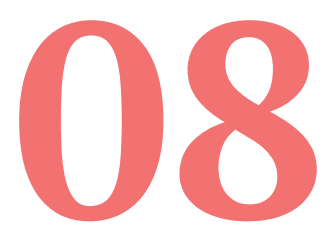

General Discussion 



\section{SCOPE OF THE THESIS}

The World Health Organization (2017) defines rehabilitation as:

An integrative strategy that aims at empowering persons with health conditions who are experiencing or are likely to experience disability so that they can achieve and maintain optimal functioning, a decent quality of life and inclusion in the community and society.

This research project focused on one particular aspect of this definition in the context of IMPT programmes: the maintenance of positive treatment gains, in order to prevent relapse. In line with this definition, we consider that effective treatment is not only determined by the level of functioning and wellbeing at the end of the programme, but also by the ability of patients to transfer key principles to their own personal situations and to retain them over time. The main rationale for starting this research project was the premise that this topic has been neglected for decades, combined with empirical indications that relapse is likely to occur after chronic pain rehabilitation (Morley, 2008; Turk \& Rudy, 1991; Wood \& Neal, 2016). An additional reason to explore this topic is the rising popularity of self-management interventions. These aim to increase self-efficacy and to support patients in actively and autonomously coping with their chronic disease (Van de Velde et al., 2019). Given the absence of studies directly related to relapse prevention after chronic pain treatment, we expected that self-management programmes - which often include components such as action planning and problem-solving (Lorig, 2015) - would incorporate valuable insights in how to enhance maintenance of gains.

\section{SUMMARY OF MAIN FINDINGS}

Our first aim was to provide an overview of the current state of research on treatment characteristics and longitudinal outcomes of IMPT programmes. In Chapter 2, we identified 74 patient cohorts that included at least a baseline and one follow-up measurement at 12 months post-treatment. This allowed us to analyse the longitudinal development of nine different outcome measures. The large majority of the calculated pre-post contrasts across these measures indicated that participation in IMPT programmes is associated with improvements in wellbeing. Importantly, in most cohorts this improvement is maintained over time. This finding differs from the typical triangular relapse patterns that have been observed in other health behaviour change interventions (Wood \& Neal, 2016). Furthermore, the data extraction procedure that was based on current standards of outcome reporting (Hoffmann et al., 2014) provided insight into the various study, patient selection and treatment approaches. By using the TIDieR checklist, we were able to 
show in detail the variation between intervention characteristics in different studies. The overview also gave rise to several suggestions for improvements in descriptions of IMPT programmes in future studies, such as the extent by which a treatment could be tailored to individual needs (reported in $47 \%$ of the cohorts), or the average duration of chronic pain of the study sample (reported in 46\%). Inspired by the work of Boutron and colleagues (2020) and Elliot and colleagues (2014, 2017) - which included suggestions for methodology and dissemination - we decided to transform this study into a living systematic review. In Chapter 3, we proposed a workflow and discussed how a living systematic review could reduce the investment of effort and time needed to update the study. Importantly, the increased speed of inclusion of primary studies into meta-analyses is expected to contribute to faster dissemination and implementation of research findings in clinical practice.

Our second aim was to develop an intervention that facilitates the maintenance of treatment gains and insights, using a co-design approach. The first step was to collect and evaluate RCTs that included generic self-management interventions for patients with chronic musculoskeletal pain (Chapter 4). Although some comparisons indicated statistically significant results favouring the self-management condition over control, clinical relevance was low. Furthermore, GRADE outcome assessments indicated low-to-moderate quality of evidence across the outcome domains. The descriptive analysis of the behaviour change techniques reported in this thesis showed a large variation between studies, indicating differences in beliefs and underlying ideas of how best to facilitate health behaviour change. This absence of clear guidance towards maintenance was in line with our preliminary literature searches on relapse in chronic pain treatment and further justified our co-design approach to intervention development. This 18-month co-design project is described in Chapter 5. To compensate for the paucity of studies on preventing relapse after IMPT programmes, we combined the different perspectives of patients, health care providers, scientists, and designers to find solutions to the problem of improving maintenance after treatment. Compared with traditional intervention development approaches, we expected that this structured collaboration with end-users would promote the acceptability of the intervention, and improve integration into existing treatment practices. Through various design iterations, we developed an evidence base, tested ideas, and developed two prototype interventions that were combined in one paper workbook. By reflecting on the whole process, we concluded that generative techniques helped to provoke detailed accounts of respondents' experiences and beliefs. Moreover, the co-design activities stimulated active engagement and equality between patients, health care providers, designers, students and researchers. However, the limited involvement of patients and health care providers in the 'develop' phase, compared with previous phases, may have affected the acceptability of the final prototype. This workbook intervention was tested during a qualitative study - described in Chapter 6 - where patients and health care providers who had used the 
workbook in the context of IMPT programmes were interviewed to discuss its feasibility. The 'insight cards' intervention was found easy to learn and apply in a clinical context, and was expected to benefit all patients. The concept of value-based goals was more difficult for patients to understand and did not always fit into the treatment programme. Important lessons learned included patients' preference for a digital version of the interventions, and the recommendation to improve alignment between the intervention content and existing treatment activities, for example, by rethinking the goal-setting procedure. A major caveat in this study was the limited sample size. We believe that this was due to the absence of clear instructions on when and how to use the workbook and the organizational changes of one of the participating treatment teams during the feasibility study. We therefore made several adjustments to the prototype in a subsequent field test that was performed in the context of a master's thesis (Wittkämper, 2018). These adjustments included a modified value-based goal-setting procedure, a more extensive training session for health care providers, and a brief treatment manual for health care professionals on how and when to use the workbook. Although the results of this intermediate step are not included in any of the previous chapters, the modifications were incorporated in the prototype workbook and further refined in the design of the mHealth application and the RCT protocol.

In the final phase of this project, we performed a new development iteration to transform the workbook into an mHealth application. We used think-aloud procedures, co-creation sessions and digital mock-ups to integrate stakeholder input. The intervention components from the paper prototype were enhanced with additional features, such as the possibility of adding photos to the insight cards and creating calendar reminders for activities within the value-based goal-setting procedure. We also added a new educational module to the application, providing the opportunity for each treatment team to add educational materials or video clips. To evaluate the effectiveness, efficiency and usability of the intervention, we developed a trial protocol (outlined in Chapter 7).

\section{IMPLICATIONS FOR CLINICAL PRACTICE}

\section{IMPT Practice}

Taken together, these findings provide several directions for clinical practice. First, this project is an example of how behaviour change strategies can be combined with IMPT programmes. This development direction helps to broaden the scope of treatment beyond achieving optimal functioning, towards maintaining positive treatment gains over time, in line with the WHO concept of rehabilitation (OECD et al., 2017). Importantly, the health psychology models that we used during the intervention development for deriving behaviour change principles can also help to further support behaviour change in current 
treatment programs. For example, Papies (2016) introduced a framework specifically designed to take unintentional effects on behaviour into account (e.g. an automatically triggered response in an associated environmental context). A more stepwise and methodical approach to optimize behaviour change has been developed by Michie and colleagues (2011). This 'Behaviour Change Wheel', helps to determine what needs to change for pre-specified outcomes to occur and to identify specific intervention components that support this change. This model has already been applied in other rehabilitation contexts to further refine intervention components (e.g. Connell et al., 2016). These ideas also fit the recently developed classification system to characterize rehabilitation treatments - the Rehabilitation Treatment Specification System (RTSS). This framework combines general behaviour change principles, such as habit formation, with rehabilitation treatment components, and aims to open the black box of rehabilitation interventions by clarifying targets for treatment, active ingredients and dosing parameters (Hart et al., 2019; Whyte et al., 2019).

The second suggestion for clinical practice is to take stock of the existing heterogeneity within treatment programmes. Although we were able to discern to a certain degree different treatment modalities from the study reports in Chapter 2, the specific treatment content as well as the underlying mechanisms were often not provided. We believe that this is problematic for evidence-based practice, because higher levels of detail are crucial to distinguish homogeneous clusters of studies for meaningful meta-analyses. A suggestion for improvement for IMPT programmes would be to more clearly describe and classify the intervention procedures, assisted by such frameworks as the RTSS and the TIDieR checklist (Dijkers, 2019; Hart et al., 2014; Hoffmann et al., 2014). Sharing this information between facilities would be an opportunity to learn from the existing heterogeneity and to promote refinement of procedures. It would also help to better distinguish certain approaches from others and check to what extent a programme conforms to the biopsychosocial model. This would further help working towards a common understanding of IMPT programmes (Kaiser et al., 2017).

Third, the large variation in patient characteristics between and within studies (identified in Chapters 2 and 4) hinders the meaningful interpretation of summary statistics. This aligns with the observations of Smeets (2021) and Eccleston \& Crombez (2017) who acknowledge the idiosyncratic nature of pain management and recommend using more single-case studies to better understand this process. For rehabilitation practice, an appropriate example would be the use of ecological momentary assessment (EMA) to monitor changes in key outcomes over time in individual patients. This type of methodology is characterized by multiple assessments over time, capturing the current state of a particular outcome for a subject in a real-world context (Shiffman et al., 2008). EMA not only enhances the 
ecological validity of the measurements, but also has the potential to provide insight in the dynamic interplay of individual symptoms and to provide continuous feedback on treatment processes (Doherty et al., 2020). In the context of preventing relapse, EMA may also be interesting as a form of remote screening at follow-up that monitors the change over time.

\section{mHealth Applications in Interdisciplinary Pain Treatment}

The decision to develop the paper workbook into an mHealth intervention reflects increased interest in digital applications in rehabilitation care. Several authors suggest that second line treatment services are likely to be transformed into some form of blended care in the coming years, suggesting an important role for evidence-based digital applications to support self-management and maintenance (de Biase et al., 2020; Eccleston et al., 2021; van Ommen et al., 2018). Restrictions on direct patient contact as a result of COVID-19 have further catalysed this development (Eccleston et al., 2020; Emerick et al., 2020). In response to this growth, expert recommendations have been developed to critically monitor implementation of these applications in rehabilitation and ensure appropriate usage. For clinicians, it is important to weigh the potential benefits and limitations for individual patients and always allow for the possibility of non-digital alternatives (Eccleston et al., 2020; Emerick et al., 2020), while improving the accessibility of the application for all patients. This is of particular importance for patients with low health literacy who often have difficulties understanding instruction texts and navigating through the interface (Kim \& Xie, 2017), threatening low adaptation and adherence. Ways of increasing accessibility include the use of videos and combining formal with informal teaching methods (Conard, 2019). Although we did not explicitly design the application for patients with low health literacy, we included a feature for various forms of educational materials and videos. Furthermore, the guideline for health care providers includes a stage in which they carefully consider the appropriateness of the intervention for the patient at that time.

\section{IMPLICATIONS FOR RESEARCH}

In the following section, we have organized the implications for research, comparisons with other studies, and future directions of this project in three main themes: 1. towards a living evidence ecosystem; 2 . the role of self-management interventions in chronic pain treatment; 3. co-design methodology in the development of health care interventions.

\section{Towards a Living Evidence Ecosystem}

According to Chambers (2018), an important challenge for the field of pain research is to decrease the time before research findings are disseminated to clinical practice. By means 
of regular updates, a living review provides the opportunity to accelerate this process. Disseminating findings via a web application also helps to increase the accessibility of data to patients, researchers, clinicians, policy makers, and guideline developers (e.g. Li, 2020; Salehi et al., 2021; Wissel et al., 2020). Although the systematic review in Chapter 2 does not include a Grading of Recommendations Assessment, Development and Evaluation (GRADE) approach to summary of findings or concrete practice guidelines, the project can be extended in that direction in several ways.

First, the dashboard can be expanded with additional modules that improve the description, interpretation and understanding of the dataset. For example, living network meta-analysis is a methodology increasingly adopted in systematic reviews. Benefits include the possibility of combining direct comparisons (within-study) with indirect comparisons (different treatments between studies) in a single analysis, resulting in a complete presentation of all available evidence (Molloy et al., 2018; Vandvik et al., 2016). These types of analyses have been successfully incorporated in other living review projects (e.g. Créquit et al., 2020).

Second, within each module, updates could include improved interaction and usability. More extensive filtering and organization options would allow users to create subgroups or to compare specific studies, based on their own interest. Another possibility is to extend existing modules with additional analyses, such as extra forest plots of standardized mean differences for studies that include control groups, or by incorporating updated reporting standards (Negrini et al., 2020).

Third, it is possible to extend the dataset itself by adding outcome measures, such as fatigue, or by including more patient groups. For example, in the protocol for the living systematic review, we propose aligning the patient criteria with the ICD-11 definition of chronic primary pain, which could result in the inclusion of more trials. Importantly, GitHub version control and the workflow that prescribes that all changes will be submitted as protocol amendments ensures transparency and replicability throughout all stages of this project. Figure 1 depicts the current organization of the application and the various levels by which it could be extended in the future.

The proposed workflow, combined with the aforementioned possibilities and possible future steps, indicates how a systematic review can be transformed into a living evidence synthesis community (Ravaud et al., 2020). Although it is not certain that this project will be the foundation of such an enterprise, it does provide insight into its feasibility and the actions required. One additional step towards integration of scientific evidence into clinical practice would be to use the outcomes from the analyses to continuously inform clinical practice guidelines. This would require living summary tables - for evaluating the 
magnitude of effect, clinical relevance and the confidence in the results of each particular outcome of interest - and a living guideline panel that could be consulted when there is a change in the outcome (Akl et al., 2017).

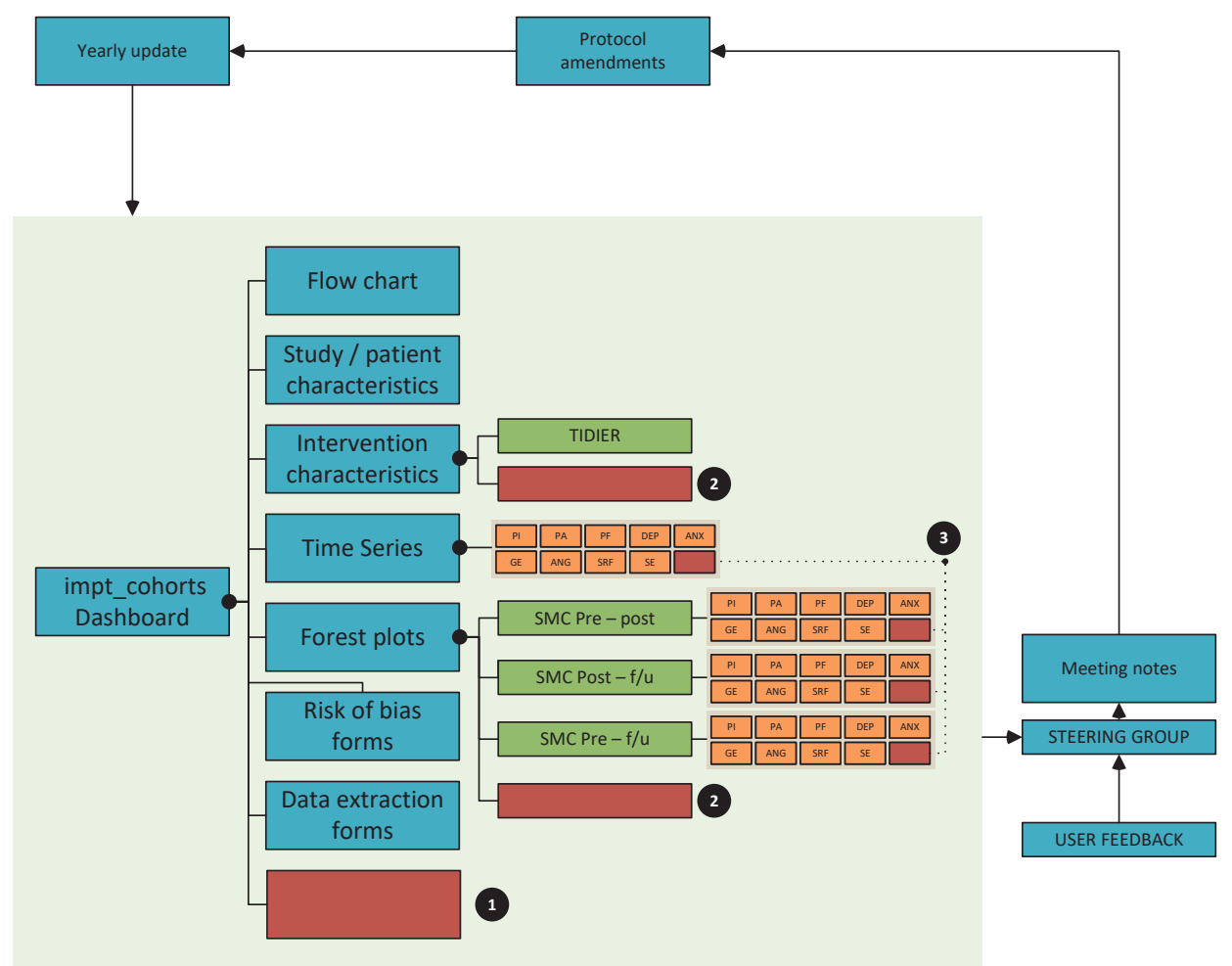

Figure 1. Infrastructure of the Web Application. Red Elements Indicate Possible Extensions of the Application.

\section{Self-Management Interventions and Chronic Pain}

Since the publication of our study on self-management interventions in 2018, we have identified multiple studies that shed new light on self-management for chronic pain. Martinez-Calderon et al. (Martinez-Calderon et al., 2020) investigated the effectiveness of various interventions on pain self-efficacy, reporting that self-management interventions showed no improvement in pain self-efficacy at 12-month follow-up, compared to control conditions, which differs from the small effect that we report in Chapter 4 . This may be explained by differences in patient inclusion criteria and ideas about the definition of selfmanagement interventions. More specifically, the eligibility criteria of our study required an education and training component, whereas Martinez-Calderon and colleagues focused 
solely on educational self-management interventions. To understand these differences and to provide more clarity on what constitutes self-management, a concept analysis was recently published (Van de Velde et al., 2019). These authors reviewed self-management studies, identifying 10 different attributes considered to be unequivocal features of selfmanagement. The eligibility criteria for our systematic review correspond to some of these attributes, explicitly emphasizing active participation (Attribute 1) and personal skills training (Attribute 9). One difference of our study compared to the concept analysis of Van de Velde and colleagues was that we excluded studies focusing on training diseasespecific skills, while they considered medical management (Attribute 10.1) to be a part of self-management. For both primary intervention studies and systematic reviews, these attributes will help clarify which specific types of interventions are included.

Two systematic reviews investigated self-management using eHealth applications. Du and colleagues (2020) analysed the short-term effects on pain intensity and disability of eight RCTs that included eHealth self-management interventions for patients with low back pain. At short-term follow-up, they reported moderate quality evidence for a positive effect on pain intensity and low quality evidence for no effect on disability. Unfortunately, none of the included studies reported long-term outcomes. Devan and colleagues (2019) systematically assessed 19 e-health applications designed to facilitate self-management support for patients with chronic pain, mapping the included mechanisms and intervention content. Similar to the results of our review, the content of the interventions greatly varied, suggesting different theories of the concept of self-management. Their analysis also indicated that none of the self-management applications had been tested in patients with pain and only a few offered the possibility of individual goal-setting. Given the importance of goal-setting and self-regulation in the context of self-managing a chronic condition (de Ridder et al., 2008; Van De Velde et al., 2019), this suggests that the workbook and mHealth application that we developed in this project address an important gap.

\section{Co-Design}

By adopting co-design methods, we aimed to involve and empower patients and healthacare providers throughout all stages of the intervention development project. This ambition is in line with current trends of increased public involvement in scientific research, such as the opportunity for the public to shape the Dutch National Research Agenda (NWO, 2021) or guidelines for involving citizens in research projects (Hickey et al., 2018). Williams and colleagues (2020) outline two different types of rationales for public participation in health research.

First, a democratic rationale relates to the idea that allocating scarce public resources to clinical research will always result in some form of prioritization. To determine these 
priorities, it is not only researchers but also other citizen representatives who ought to have influence in this process. This would require the adoption of co-design approaches in research projects. When evaluating this project from a democratic perspective, we consider the project planning and the extent of generative techniques as particular strengths. By ensuring that co-design was spread over the entire duration of the 2-year project, we created touchpoints for end-users to exert influence at each development stage. Although this is a necessary condition, it is not sufficient to guarantee democratic co-design. According to Williams and colleagues (2020), “...having a seat at the meeting table does not guarantee an opportunity or the confidence to speak up, or that contributions made are officially recorded in meeting minutes, let alone give rise to the tangible influence on services or research". Indeed, there are signs that public involvement in health research can be narrowed down to limited influence on the project, ticking the box of public participation at minimal cost (Martin et al., 2018; Robert et al., 2020; Williams et al., 2020). Thus, it is important not only to involve stakeholders but also to actively empower them to express their experiences and ideas. The various generative techniques (methods, exercises and assignments to elicit implicit knowledge and latent needs), combined with the specific experiences of co-design researchers (who planned and facilitated each co-design activity), were important to ensure actual impact of the participants. A particular weakness in this respect was that time pressure in the final stage of the workbook development resulted in limited involvement of the patient population in that phase.

Second, a technocratic rationale refers to the belief that other stakeholders have unique insights related to the problem under investigation that may improve the outcome of the project. As we outlined in Chapter 5, the final prototype and the AGRIPPA application were thoroughly based on the contributions of key stakeholder groups. As initial stages of intervention developments rarely receive scientific attention and are often underreported (Hoddinott, 2015), this chapter contributes by clarifying the activities that have led to the prototype. A step in the right direction comes from a new reporting checklist for intervention development. This outcome of a Delphi study includes an item on the extent to which stakeholders contributed to the development process, encouraging an explicit description of the actual methods used to include these stakeholders (Duncan et al., 2020; Staniszewska et al., 2017). When the results of the ongoing AGRIPPA trial are analysed, we will obtain further insights into the extent to which this co-designed intervention improves maintenance of outcomes in patients with chronic pain in IMPT programmes. 


\section{LIMITATIONS}

In each chapter, we have already discussed the specific limitations of each study. In this section we will address three main overarching limitations. First, the neglect of relapse in pain research has been the main motivation to start this project. It is therefore important to reflect on what this project has yielded in this area. Although Chapter 2 did not identify overall trends in relapse in longitudinal patient cohorts, the outcome distributions of these measures still leave room for a proportion of individual patients to relapse. We attempted to estimate this proportion but we were not sufficiently confident in these estimates to report them. Consequently, we cannot provide a clear indication of the prevalence of relapse. When more individual patient data become available, it will be possible to better determine the scope of this problem. A second issue is the difficulty of determining what constitutes relapse in the context of IMPT programmes. We circumvented this by focusing on evaluating and supporting the ability to maintain positive treatment gains over time for a broad range of outcome measures. Second, the outcome measures currently used often do not sufficiently reflect meaningful endpoints for patients (Eccleston \& Crombez 2019), and vary greatly between studies. A potential solution to allow more accurate measurements is the development of core outcome sets. Although currently under development for patients who participated in IMPT programmes, they are not yet available (Pogatzki-Zahn 2019, Kaiser et al., 2018). The third limitation is that intervention development and feasibility testing were largely performed in two treatment facilities. While increasing mutual understanding and lowering the barriers for activities such as co-design, this also resulted in lower variability in health care professionals involved and in treatment contexts. The outcomes of the AGRIPPA trial should provide more clarity as to what extent this has influenced the outcomes, because that trial also includes additional treatment facilities that offer the intervention.

\section{IMPACT}

By discussing the role of the living systematic review in improving the evidence ecosystem, we have already addressed a part of this project that could have an impact in future rehabilitation science. To facilitate dissemination, we have specifically ensured that our annotated R scripts are open-access for other projects. In addition, there are three other impacts resulting from this project. We will outline these below. 


\section{The AGRIPPA Application}

In Chapter 7, we discussed the main deliverable of the second aim of this project: a first version of the AGRIPPA mHealth application. Although this application is currently only accessible for treatment facilities that are participating in the trial where its additional value is being assessed, it is possible to distribute the application further across multiple facilities. New partners can be provided with access to a personal back-end environment where they have the ability to set up their own education module for their patients, with texts, video clips or links to websites. Importantly, the developers have taken the possibility of including additional languages, facilitating potential future endeavours in other countries and increasing the accessibility of the app to non-native Dutch speakers in the Netherlands. Specific valorization and dissemination strategies are currently being discussed in the AGRIPPA consortium. Other potential development ideas include the incorporation of EMA, which is increasingly provided through smartphones (Doherty et al., 2020; Shiffman et al., 2008). For patients with chronic pain, this could help in obtaining a complete and accurate insight into the impact of pain in their personal lives (May et al., 2018). Furthermore, the inclusion of options for communicating with the treatment team to facilitate a form of blended care could enhance the application. This facilitates the possibility of tailoring IMPT treatments in more flexible and efficient ways as regards the needs of patients in terms of time and place. Integration of all these services into one platform would help to bring all information sources together, which is likely to improve usability. Another possible direction is to explore the potential of the application in other domains. The value-based goals module and the insight cards are not specific to pain rehabilitation and could be adapted to support self-regulation for a range of chronic conditions. Value-based goal-setting has even been successfully tested in high schools (Chase et al., 2013), indicating the wide potential of these instruments.

\section{Towards an Integrative Model of Clinical Reasoning and Behaviour Change}

During the development of our intervention, we used the persuasive-by-design model to explore barriers and facilitators for maintenance of treatment gains. This model was originally created to help design professionals with no expertise in behaviour change to design evidence-based health interventions (Hermsen et al., 2014). The model includes five different perspectives or 'behavioural lenses' that each highlight a different aspect of behaviour regulation (e.g., habits and impulses). Any target behaviour can be evaluated through each 'lens' to identify potential barriers or facilitators for effective regulation. Although we used this model to develop generic interventions that could benefit all patients, we believe that it has the potential to assist health care professionals in developing maintenance strategies for individual patients. This corresponds to the recommendations 
of various researchers to tailor pain treatments to individual patient needs (Eccleston \& Crombez, 2017; Vlaeyen \& Crombez, 2020). Through various student projects, we are currently evaluating the usability and clinical potential of the persuasive-by-design model as an instrument for tailoring behaviour change strategies in a clinical setting. The first results indicate that the model contributes positively to the integration of behaviour change techniques into clinical practice and clinical reasoning (Beekhuizen, 2018; Gilhuis, 2018; Schaefers, 2020).

\section{Transformative Effect on the Research Network}

The final area of impact concerns the increased collaboration between the research network partners. This project started with the SOLACE project, funded by SIA RAAK (2014-01-23P). The key objective of this Dutch public funding agency is to stimulate strategic partnerships between universities and other public institutions to collaborate on important social issues (Regieorgaan \& SIA, n.d.). Conforming with this ideal, the consortium partners have regularly collaborated in subsequent projects, further strengthening the links in this network. The current state is a form of ongoing project-based collaboration, with a specific focus on translating research findings to optimize clinical practice. The recurring central role of co-design within these projects indicates an increased interest in the inclusion of stakeholders and end-users into the research design. This transformative impact does not only fit the aims of SIA RAAK but is also been considered as one of the main ambitions of co-design research in general (Robert et al., 2020). 


\section{REFERENCES}

Akl, E. A., Meerpohl, J. J., Elliott, J., Kahale, L. A., Schünemann, H. J., Agoritsas, T., Hilton, J., Perron, C., Hodder, R., Pestridge, C., Albrecht, L., Horsley, T., Platt, J., Armstrong, R., Nguyen, P. H., Plovnick, R., Arno, A., Ivers, N., Quinn, G., ... Pearson, L. (2017). Living systematic reviews: 4. Living guideline recommendations. J Clin Epidem, 91, 47-53. https://doi.org/10.1016/j.jclinepi.2017.08.009

Beekhuizen, R. Q. (2018). kinderfysiotherapeuten met de Behavioural Action Tool Een kwalitatief onderzoek [pedeatric physiotherapists using the Behavioural action tool: a qualitative study]. Univeristy of Applied Sciences Utrecht.

Boutron, I., Créquit, P., Williams, H., Meerpohl, J., Craig, J. C., \& Ravaud, P. (2020). Future of evidence ecosystem series: 1. Introduction Evidence synthesis ecosystem needs dramatic change. J Clin Epidem, 123, 135-142. https://doi.org/10.1016/j.jclinepi.2020.01.024

Chambers, C. T. (2018). From evidence to influence: dissemination and implementation of scientific knowledge for improved pain research and management.Pain,159,S56-S64.https://doi.org/10.1097/j.pain.0000000000001327

Chase, J. A., Houmanfar, R., Hayes, S. C., Ward, T. A., Vilardaga, J. P., \& Follette, V. (2013). Values are not just goals: Online ACT-based values training adds to goal setting in improving undergraduate college student performance. J Context Behav Sci, 2(3-4), 79-84. https://doi.org/10.1016/j.jcbs.2013.08.002

Conard, S. (2019). Best practices in digital health literacy. Int J Card, 292, 277-279. https://doi.org/10.1016/j. ijcard.2019.05.070

Connell, L. A., McMahon, N. E., Tyson, S. F., Watkins, C. L., \& Eng, J. J. (2016). Mechanisms of action of an implementation intervention in stroke rehabilitation: a qualitative interview study. BMC Health Servic Res, 16(1), 1-10. https://doi.org/10.1186/s12913-016-1793-8

Créquit, P., Boutron, I., Meerpohl, J., Williams, H. C., Craig, J., \& Ravaud, P. (2020). Future of evidence ecosystem series: 2. current opportunities and need for better tools and methods. J Clin Epidem, 123, 143-152. https://doi. org/10.1016/j.jclinepi.2020.01.023

de Biase, S., Cook, L., Skelton, D. A., Witham, M., \& ten Hove, R. (2020). The COVID-19 rehabilitation pandemic. Age Ageing, 49(5), 696-700. https://doi.org/10.1093/ageing/afaa118

de Ridder, D., Geenen, R., Kuijer, R., \& van Middendorp, H. (2008). Psychological adjustment to chronic disease. Lancet, 372(9634), 246-255. https://doi.org/10.1016/S0140-6736(08)61078-8

Devan, H., Farmery, D., Peebles, L., \& Grainger, R. (2019). Evaluation of self-management support functions in apps for people with persistent pain: Systematic review. JMIR MHealth and UHealth, 7(2), 1-15. https://doi. org/10.2196/13080

Dijkers, M. P. (2019). An end to the black box of rehabilitation? Arch Phys Med Rehabil, 100(1), 144-145. https://doi. org/10.1016/j.apmr.2018.09.108

Doherty, K., Balaskas, A., \& Doherty, G. (2020). The design of ecological momentary assessment technologies. Interact Comp, 32(3), 257-278. https://doi.org/10.1093/iwcomp/iwaa019

Du, S., Liu, W., Cai, S., Hu, Y., \& Dong, J. (2020). The efficacy of e-health in the self-management of chronic low back pain: A meta analysis. Int J Nur Stud, 106, 103507. https://doi.org/10.1016/j.ijnurstu.2019.103507

Duncan, E., O’Cathain, A., Rousseau, N., Croot, L., Sworn, K., Turner, K. M., Yardley, L., \& Hoddinott, P. (2020). Guidance for reporting intervention development studies in health research (GUIDED): An evidence-based consensus study. BMJ Open, 10(4), 1-12. https://doi.org/10.1136/bmjopen-2019-033516

Eccleston, C., Blyth, F. M., Dear, B. F., Fisher, E. A., Keefe, F. J., Lynch, M. E., Palermo, T. M., Reid, M. C., \& Williams, A. C. D. C. (2020). Managing patients with chronic pain during the COVID-19 outbreak: considerations for the rapid introduction of remotely supported (eHealth) pain management services. Pain, 161(5), 889-893. https:// doi.org/10.1097/j.pain.0000000000001885 
Eccleston, C., \& Crombez, G. (2017). Advancing psychological therapies for chronic pain. F1000Research, 6(461). https://doi.org/10.12688/f1000research.10612.1

Eccleston, C., Fisher, E., Howard, R. F., Slater, R., Forgeron, P., Palermo, T. M., Birnie, K. A., Anderson, B. J., Chambers, C. T., Crombez, G., Ljungman, G., Jordan, I., Jordan, Z., Roberts, C., Schechter, N., Sieberg, C. B., Tibboel, D., Walker, S. M., Wilkinson, D., \& Wood, C. (2021). Delivering transformative action in paediatric pain: a Lancet Child \& Adolescent Health Commission. Lancet Child Adol Health, 5(1), 47-87. https://doi.org/10.1016/S23524642(20)30277-7

Elliott, J. H., Synnot, A., Turner, T., Simmonds, M., Akl, E. A., McDonald, S., Salanti, G., Meerpohl, J., MacLehose, H., Hilton, J., Tovey, D., Shemilt, I., Thomas, J., Agoritsas, T., Perron, C., Hodder, R., Pestridge, C., Albrecht, L., Horsley, T., ... Pearson, L. (2017). Living systematic review: 1. Introduction-the why, what, when, and how. J Clin Epidem, 91, 23-30. https://doi.org/10.1016/j.jclinepi.2017.08.010

Elliott, J. H., Turner, T., Clavisi, O., Thomas, J., Higgins, J. P. T., Mavergames, C., \& Gruen, R. L. (2014). Living systematic reviews: An emerging opportunity to narrow the evidence-practice gap. PLoS Medicine, 11(2), 1-6. https://doi. org/10.1371/journal.pmed.1001603

Emerick, T., Alter, B., Jarquin, S., Brancolini, S., Bernstein, C., Luong, K., Morrisseyand, S., \& Wasan, A. (2020). Telemedicine for chronic pain in the COVID-19 era and beyond. Pain Med, 21(9), 1743-1748. https://doi. org/10.1093/pm/pnaa220

Gilhuis, L. (2018). De Behavioural Action Tool onder de loep genomen door fysiotherapeuten Een kwalitatief onderzoek [The Behavioral Action Tool through the lens of physiotherapists: a qualitative study]. Univeristy of Applied Sciences Utrecht.

Hart, T., Dijkers, M. P., Whyte, J., Turkstra, L. S., Zanca, J. M., Packel, A., Van Stan, J. H., Ferraro, M., \& Chen, C. (2019). A theory-driven system for the specification of rehabilitation treatments. Arch Phys Med Rehabil, 100(1), 172180. https://doi.org/10.1016/j.apmr.2018.09.109

Hart, T., Tsaousides, T., Zanca, J. M., Whyte, J., Packel, A., Ferraro, M., \& Dijkers, M. P. (2014). Toward a theory-driven classification of rehabilitation treatments. Arch Phys Med Rehabil, 95(1), S33-S44. https://doi.org/10.1016/j. apmr.2013.05.032

Hermsen, S., Renes, R. J., \& Frost, J. (2014). Persuasive by Design: a model and toolkit for designing evidencebased interventions. In J. van Leeuwen, P. J. Stappers, M. H. Lamers, \& M. J. M. R. Thissen (Eds.), Creating the difference: Proceedings of the chi sparks 2014 conference (pp. 74-77). The Hague University of Applied Sciences. http://proceedings.chi-sparks.nl

Hickey, G., Brearley, S., Coldham, T., Denegri, S., Green, G., Staniszewska, S., Tembo, D., Torok, K., \& Turner, K. (2018). Guidance on co-producing a research project. In NIHR Involve. INVOLVE.

Hoddinott, P. (2015). A new era for intervention development studies. Pilot and Feasibility Studies, 1(1), 1-4. https:// doi.org/10.1186/s40814-015-0032-0

Hoffmann, T., Glasziou, P., Boutron, I., Milne, R., Perera, R., Moher, D., Altman, D. G., Barbour, V., Macdonald, H., Johnston, M., Lamb, S., Dixon-Woods, M., McCulloch, P., Wyatt, J., Chan Pelan, A., \& Michie, S. (2014). Better reporting of interventions: Template for intervention description and replication (TIDieR) checklist and guide. BMJ, 20(12), 1439-1448. https://doi.org/10.1136/bmj.g1687

Kaiser, U., Treede, R. D., \& Sabatowski, R. (2017). Multimodal pain therapy in chronic noncancer pain-gold standard or need for further clarification? Pain, 158(10), 1853-1859. https://doi.org/10.1097/j.pain.0000000000000902

Kim, H., \& Xie, B. (2017). Health literacy in the eHealth era: A systematic review of the literature. Pat Educ Couns, 100(6), 1073-1082. https://doi.org/10.1016/j.pec.2017.01.015

Li, Y. (2020). Towards fast prototyping of cloud-based environmental decision support systems for environmental scientists using R Shiny and Docker. Env Model Sofware, 132. https://doi.org/10.1016/j.envsoft.2020.104797 
Lorig, K. (2015). Chronic Disease Self-Management Program: Insights from the Eye of the Storm. Front Pub Health, 2 , 253. https://doi.org/10.3389/fpubh.2014.00253

Martin, G. P., Carter, P., \& Dent, M. (2018). Major health service transformation and the public voice: conflict, challenge or complicity? J Health Serv Res Pol, 23(1), 28-35. https://doi.org/10.1177/1355819617728530

Martinez-Calderon, J., Flores-Cortes, M., Morales-Asencio, J. M., Fernandez-Sanchez, M., \& Luque-Suarez, A. (2020). Which interventions enhance pain self-efficacy in people with chronic musculoskeletal pain? A systematic review with meta-analysis of randomized controlled trials, including over 12000 participants. J Orth Sports Phys Ther, 50(8), 418-430. https://doi.org/10.2519/jospt.2020.9319

May, M., Junghaenel, D. U., Ono, M., Stone, A. A., \& Schneider, S. (2018). Ecological Momentary Assessment Methodology in Chronic Pain Research: A Systematic Review. J Pain, 19(7), 699-716. https://doi.org/10.1016/j. jpain.2018.01.006

Michie, S., van Stralen, M. M., \& West, R. (2011). The behaviour change wheel: A new method for characterising and designing behaviour change interventions. Implement Sci, 6(1), 42. https://doi.org/10.1186/1748-5908-6-42

Molloy, G. J., Noone, C., Caldwell, D., Welton, N. J., \& Newell, J. (2018). Network meta-analysis in health psychology and behavioural medicine: a primer. Health Psychol Rev, 12(3), 254-270. https://doi.org/10.1080/17437199.20 18.1457449

Morley, S. (2008). Relapse prevention: Still neglected after all these years. Pain, 134(3), 239-240. https://doi. org/10.1016/j.pain.2007.12.004

Negrini, S., Armijo-Olivo, S., Patrini, M., Frontera, W. R., Heinemann, A. W., MacHalicek, W., Whyte, J., \& Arienti, C. (2020). The randomized controlled trials rehabilitation checklist: Methodology of development of a reporting guideline specific to rehabilitation. Am J Phys Med Rehabil, 99(3), 210-215. https://doi.org/10.1097/ PHM.0000000000001370

NWO. (2021). Nationale Wetenschapsagenda (NWA) | NWO. https://www.nwo.nl/onderzoeksprogrammas/nationalewetenschapsagenda-nwa

OECD, Eurostat, \& WHO. (2017). A System of Health Accounts 2011: revised edition (pp. 71-119). OECD Publishing. https://doi.org/https://doi.org/10.1787/9789264270985-en.

Papies, E. K. (2016). Health goal priming as a situated intervention tool: how to benefit from nonconscious motivational routes to health behaviour. Health Psychol Rev, 10(4), 408-424. https://doi.org/10.1080/174371 99.2016.1183506

Ravaud, P., Créquit, P., Williams, H. C., Meerpohl, J., Craig, J. C., \& Boutron, I. (2020). Future of evidence ecosystem series: 3. From an evidence synthesis ecosystem to an evidence ecosystem. J Clin Epidem, 123, 153-161. https:// doi.org/10.1016/j.jclinepi.2020.01.027

Regieorgaan, \& SIA. (n.d.). Over ons - Regieorgaan SIA. 2021. Retrieved April 12, 2021, from https://regieorgaan-sia. nl/over-regieorgaan-sia/over-ons/\#Netwerkorganisatie

Robert, G., Donetto, S., \& Williams, O. (2020). Co-designing Healthcare Services with Patients. In E. Loeffler \& T. Bovaird (Eds.), The palgrave handbook of co-production of public services and outcomes. Springer International Publishing. https://doi.org/10.1007/978-3-030-53705-0

Salehi, M., Arashi, M., Bekker, A., Ferreira, J., Chen, D. G., Esmaeili, F., \& Frances, M. (2021). A Synergetic R-Shiny Portal for Modeling and Tracking of COVID-19 Data. Front Pub Health, 8, 1-10. https://doi.org/10.3389/ fpubh.2020.623624

Schaefers, T. (2020). De ervaringen van fysiotherapeuten met het integreren van gedragsveranderingstechnieken, middels een prototype van de Behavioural Action Tool - een kwalitatief onderzoek [experiences of physiotherapists with using behaviour change techniques by means of . Univeristy of Applied Sciences Utrecht.

Shiffman, S., Stone, A. A., \& Hufford, M. R. (2008). Ecological Momentary Assessment. Ann Rev Clin Psychol, 4(1), 1-32. https://doi.org/10.1146/annurev.clinpsy.3.022806.091415 
Smeets, R. J. E. M. (2021). How to sustain and improve client centred (matched) care in chronic musculoskeletal pain? Start by changing the way policy makers select and judge the large amount of available data, and get rid of the dogma of stepped care. Eur J Physiother, O(0), 1-2. https://doi.org/10.1080/21679169.2021.1888469

Staniszewska, S., Brett, J., Simera, I., Seers, K., Mockford, C., Goodlad, S., Altman, D. G., Moher, D., Barber, R., Denegri, S., Entwistle, A., Littlejohns, P., Morris, C., Suleman, R., Thomas, V., \& Tysall, C. (2017). GRIPP2 reporting checklists: Tools to improve reporting of patient and public involvement in research. BMJ (Online), 358. https:// doi.org/10.1136/bmj.j3453

Turk, D. C., \& Rudy, T. E. (1991). Neglected topics in the treatment of chronic pain patients - relapse, noncompliance, and adherence enhancement. Pain, 44(1), 5-28. https://doi.org/10.1016/0304-3959(91)90142-K

Van De Velde, D., De Zutter, F., Satink, T., Costa, U., Janquart, S., Senn, D., \& De Vriendt, P. (2019). Delineating the concept of self-management in chronic conditions: A concept analysis. BMJ Open, 9(7). https://doi.org/10.1136/ bmjopen-2018-027775

van Ommen, B., Wopereis, S., van Empelen, P., van Keulen, H. M., Otten, W., Kasteleyn, M., Molema, J. J. W., de Hoogh, I. M., Chavannes, N. H., Numans, M. E., Evers, A. W. M., \& Pijl, H. (2018). From diabetes care to diabetes cure-the integration of systems biology, ehealth, and behavioral change. Front Endocrin, 8, 381. https://doi. org/10.3389/fendo.2017.00381

Vandvik, P. O., Brignardello-Petersen, R., \& Guyatt, G. H. (2016). Living cumulative network meta-analysis to reduce waste in research: A paradigmatic shift for systematic reviews? BMC Medicine, 14(1), 4-6. https://doi. org/10.1186/s12916-016-0596-4

Vlaeyen, J. W. S., \& Crombez, G. (2020). Behavioral conceptualization and treatment for chronic pain. Ann Rev Clin Psychol, 16, 187-212. https://doi.org/10.1037/h0100082

Whyte, J., Dijkers, M. P., Hart, T., Van Stan, J. H., Packel, A., Turkstra, L. S., Zanca, J. M., Chen, C., \& Ferraro, M. (2019). The importance of voluntary behavior in rehabilitation treatment and outcomes. Arch Phys Med Rehabil, 100, 156-163. https://doi.org/10.1016/j.apmr.2018.09.111

Williams, O., Robert, G., Martin, G. P., Hanna, E., \& O’Hara, J. (2020). Is Co-production just really good PPI? Making sense of patient and public involvement and co-production networks. In: Bevir M., Waring J. (eds) Decentring Health and Care Networks. Organizational Behaviour in Healthcare (pp 213 -237). Palgrave Macmillan, Cham. https://doi-org /10.1007/978-3-030-40889-3_10

Wissel, B. D., Van Camp, P. J., Kouril, M., Weis, C., Glauser, T. A., White, P. S., Kohane, I. S., \& Dexheimer, J. W. (2020). An interactive online dashboard for tracking COVID-19 in U.S. counties, cities, and states in real time. J Am Med Informatics Ass, 27(7), 1121-1125. https://doi.org/10.1093/jamia/ocaa071

Wittkämper, D. (2018). Ervaringen van behandelaren met het toepassen van het Werkboek Solace als hulpmiddel binnen een chronisch pijn behandeltraject: een focusgroep studie [experiences of healthcare professionals in applying the SOLACE workbook in a pain rehabilitation program. University of Applied Sciences Utrecht.

Wood, W., \& Neal, D. T. (2016). Healthy through habit: Interventions for initiating \& maintaining health behavior change. Behav Sci Pol, 2(1), 71-83. https://doi.org/10.1353/bsp.2016.0008 




\section{SUMMARY}

Chronic pain is one of the leading causes of worldwide disability. Unfortunately, there is often no treatment option available that directly influences the pain itself. Instead, biopsychosocial approaches that aim to decrease disability and optimize self-management, wellbeing and daily life functioning are considered treatments of choice. Since their introduction in the 1950s, the so-called Interdisciplinary Multimodal Pain Treatment (IMPT) programmes have become increasingly popular and spread globally. The programmes are provided by a team of health care providers with different professional backgrounds who continuously collaborate to deliver comprehensive biopsychosocial treatment.

Despite evidence that IMPT programmes have a positive effect on physical, mental and social health outcomes, there are indications that patients find it difficult to maintain positive treatment gains over time. This doctoral thesis is centred around this potential problem of relapse after successful treatment. The first part focuses on obtaining and maintaining an up-to-date overview of the current state of research literature on longitudinal outcomes of IMPT programmes. In Chapters 2 and 3, we address the following questions:

1. How does the physical, psychological and social health of patients with chronic pain who participated in IMPT programmes change over time?

2. To what extent do the included studies vary with respect to study design, patient and treatment characteristics?

3. How can we transform a regular review into a living systematic review to increase the speed of dissemination and implementation of scientific findings into clinical practice?

In Chapter 2, we describe the results of a systematic review and meta-analysis. The two main objectives of this study were a) to analyse the longitudinal outcomes of patient cohorts who participated in IMPT programmes and b) to describe the variation of study, patient, treatment and outcome characteristics in detail. We identified 65 eligible studies describing the change over time of 74 unique patient cohorts. For all these cohorts, there was at least one pre-treatment and a one-year post-treatment measurement on one of the outcomes of interest (i.e. pain interference, physical functioning, depression, anxiety, anger, emotional functioning, self-efficacy, social functioning, and pain intensity). We evaluated change over time by calculating contrasts between pre-post, post-final follow-up and pre-final followup measurements. Most of the pre-post contrasts (85\%) showed a statistically significant favourable change over time. Importantly, in the majority of these cohorts, this effect was maintained over time or even improved at final follow-up. This indicates that participation in IMPT programmes is associated with positive changes in health outcomes, which are generally maintained at follow-up. When interpreting these findings, it is important to 
remember that the contrasts are calculated with summary statistics and that favourable mean changes in cohorts still leave room for individual patients to relapse. Future studies should provide more insight into the maintenance of treatment gains at the individual patient level. For the second objective, we identified substantial variation between studies in patient inclusion criteria, treatment content, dosing parameters and outcome measures. This not only indicates the existence of different views on optimal designs for IMPT programmes but also hinders meaningful comparisons of these programmes.

A crucial challenge for systematic reviewers is keeping up with the increasing speed of publication of primary studies. To prevent our systematic review quickly going out of date, we developed a workflow for a living systematic review (LSR). This type of study is characterized by recurring updates and (semi-)automated procedures to speed up the review process. Although LSRs have been successfully implemented in rapidly developing research areas, we believe there is also potential for iteratively building an up-to-date evidence base in rehabilitation science. In Chapter 3, we outline this idea and explain the required steps to transform an existing systematic review into a living systematic review.

The second aim of this research project was to co-design an intervention with stakeholders that facilitates the maintenance of treatment gains over time. In Chapters 4-7, we address the following questions:

- What are the immediate and long-term effects of generic self-management interventions for patients with chronic musculoskeletal pain in terms of physical functioning, self-efficacy, pain intensity and physical activity?

- Which programme characteristics, components, and behaviour change techniques are included in the self-management interventions?

- To what extent do co-design practices facilitate the translation of meaningful stakeholder experiences into the design of a health care intervention?

- How can co-design contribute to stakeholder involvement, generation of relevant insights and ideas, and incorporation of stakeholder input in the design of an intervention?

- How do patients and health care providers evaluate the current workbook prototype in the context of IMPT programmes in terms of acceptability and applicability?

- How can we evaluate the effectiveness of the AGRIPPA mHealth intervention, determine its cost-effectiveness, and acquire insight into its level of engagement and usability?

This section starts with a systematic review and meta-analysis of generic selfmanagement interventions for patients with chronic musculoskeletal pain (Chapter 4). 
Self-management interventions provide patients with skills and knowledge to support the everyday management of their chronic disease. This approach may therefore hold important information on how to deal with relapse, which could be used to improve IMPT programmes. Our primary aim was to determine the effectiveness of generic selfmanagement programmes in improving physical functioning, self-efficacy, pain intensity and physical activity, compared to control. We identified 20 randomized controlled trials comparing a generic self-management intervention to a control group. For each outcome, we calculated standardized mean differences post-treatment and at follow-up, pooling the results if there was sufficient homogeneity between studies. Following a GRADE approach, we found moderate quality evidence for a statistically significant, but clinically unimportant, effect on physical functioning and pain intensity, favouring generic self-management interventions. At follow-up, we found moderate quality evidence of a small but clinically unimportant effect for self-efficacy, favouring generic self-management interventions. The other comparisons indicated no statistically significant effect. In line with our findings in Chapter 2, we found substantial variation in intervention content, especially with respect to the described behaviour change techniques. This indicates different perspectives on how to change self-management behaviours. Overall, these results indicate that generic selfmanagement interventions have a marginally favourable effect for patients with chronic pain on self-efficacy, physical functioning, and pain intensity.

Chapter 5 contains the description and systematic evaluation of a co-design project to develop a relapse prevention intervention for patients with chronic pain who had participated in IMPT programmes. In contrast to many traditional health care intervention development approaches, co-design systematically includes the perspective of end-users in each phase of the development process. Consequently, this method has the potential to increase the uptake of health interventions. The first aim of this study was to provide an overview of all activities that were employed during the development of the prototype intervention. The second aim was to reflect at each development stage on how co-design influenced stakeholder involvement, the generation of insights, and the incorporation of stakeholder input into the design. In this embedded case study, we adopted a participatory action research approach, collaborating with patients, health care providers and designers throughout the project rather than treating them only as research subjects. During the project, all researchers contributed to a qualitative dataset through individual notes and reflective sessions. We also analysed all input from the co-design activities and the steering committee meeting minutes. We used the Double Diamond Model to organize the data across four design phases (i.e. 'discover', 'define', 'develop' and 'deliver') and adopted a deductive content approach to identify information concerning our second study aim. Over the course of 18 months, we organized multiple design activities, including interviews, co-creation sessions, data-analysis activities, and prototype-building sessions. 
During these activities, we developed an evidence base, tested ideas and developed two prototype interventions that we merged into one relapse prevention workbook. In our reflection, we concluded that the generative techniques helped participants to provide a detailed description of their experiences and beliefs beyond their initial response. The co-design approach also promoted active participation and equal collaboration between the participants. Limitations included restricted involvement of patients and health care providers in the final stage of the project, potentially affecting the acceptability of the final prototype. Overall, this case study provides an example of how co-design can support the transfer of ideas, mainly derived from patients and health care providers, into a prototype intervention.

In Chapter 6, we present the results of a feasibility study of the workbook prototype in a clinical setting. The main objective was to assess the level of acceptability and the applicability of the workbook for patients and health care providers in IMPT programmes. We performed a qualitative study in two different facilities. Health care providers received a brief training session in how to use the workbook but were subsequently free to decide when the workbook would be appropriate for patients whom they treated during the six month study period. Subsequently, eleven patients and four health care providers provided input on the feasibility of the intervention by means of interviews (patients) and a focus group (health care providers). We analysed the data with a deductive thematic content approach and we clustered meaningful data segments into four themes: applicability in existing treatment programmes; acceptability of the workbook content; acceptability of the workbook form; and adaptability. Overall, the workbook was perceived as easy to use and in line with existing treatment components. The results further indicated directions for improvement, but also comprised experiences relevant to its feasibility in a rehabilitation context. However, various limitations - including a high dropout rate and a small sample size - negatively influenced the generalizability of these results. Future studies should not only focus on further revision of the intervention, but also on improvement of the implementation and data collection procedures.

One important recommendation from the feasibility study was to transform the workbook into a digital intervention. In a subsequent study project, we developed the AGRIPPA application that contained both relapse prevention strategies. The application also contained an additional information and education module where different IMPT programmes could add their own information materials, such as videos, assignments and website links. Chapter 7 is a description of a multicentre randomized controlled trial protocol with two parallel groups. In a 12-month study period, we were to recruit 158 patients in 6 treatment facilities. Patients in the experimental condition would receive the AGRIPPA mHealth application in addition to the IMPT programme. Patients in the 
control condition would only receive the standard IMPT treatment. We were to evaluate the difference in longitudinal outcomes of pain-related disability between both groups. In addition, we were to evaluate the effect of the AGRIPPA app on health care utilization and societal costs and explore to what extent usability measures are associated with a favourable change over time of pain-related disability. This study was intended to provide insight into the question of to what extent the app would influence the maintenance of treatment gains over time.

Chapter 8 contains a general discussion on the findings of this research project. With respect to clinical practice, we first stress the potential of integrating behaviour change strategies into rehabilitation programmes. Based on the two systematic reviews, we also highlight the importance of detailed intervention descriptions. Recently, multiple classification systems have become available which can guide clinicians and researchers in providing a concise overview of rehabilitation programmes, including a clear description of active ingredients, dosing parameters and tailoring options. This could help to make sense of the heterogeneity in interventions and outcomes of IMPT programmes. We next discuss the increased importance of digital applications in rehabilitation, and recommend the critical evaluation of the implementation process to ensure sufficient adaptation for all patient groups. Implications for research include further suggestions to extend the scope of the living systematic review project, for example by including additional analyses and outcomes. We further discuss the development of self-management interventions that have become available since the publication of our systematic review. With respect to co-design, we reflect on both its democratic and technocratic value in the context of intervention development projects. The concluding paragraph provides three directions for future impact: further development and dissemination of the AGRIPPA application; integration of behaviour change principles into clinical reasoning approaches; and the potential of interdisciplinary public consortia on translating research findings to clinical practice. 



\section{NEDERLANDSE SAMENVATTING (SUMMARY IN DUTCH)}

Chronische pijn is wereldwijd één van de belangrijkste oorzaken voor verminderd functioneren en gezondheidsproblemen. Er wordt geschat dat ongeveer 30\% van de bevolking leidt aan chronische pijn, waarbij er in veel gevallen sprake is van een substantiële impact op fysiek, psychisch en sociaal welzijn. De oorzaken van chronische pijn zijn complex, multifactorieel en vaak slechts beperkt gerelateerd aan lokale weefselschade. Tot op heden is er in veel gevallen geen adequate behandeling beschikbaar om van de pijn af te komen. Het alternatief is een biopsychosociale aanpak met als doel om de ervaren beperkingen in het dagelijks leven te verminderen en om ondersteuning te bieden richting een betekenisvol leven met pijn. Deze interdisciplinaire multimodale pijnrevalidatie (IMP) programma's zijn opgebouwd uit verschillende componenten, zoals pijneducatie, fysieke training en cognitieve gedragstherapie. De revalidatie wordt aangeboden door een team van verschillende zorgprofessionals die samen zorgen voor een geïntegreerde biopsychosociale aanpak waarin de persoonlijke doelen van de patiënt in de context van het dagelijks functioneren centraal staan. Hoewel er de afgelopen jaren veel bewijs is verzameld dat deze programma's een positief effect hebben op de fysieke, mentale en sociale gezondheid, zijn er ook aanwijzingen dat deelnemers van deze programma's moeite hebben om de positieve uitkomsten na afloop vast te houden. In dit proefschrift staat het probleem van terugval na succesvolle revalidatie centraal. Deel 1 bevat twee hoofdstukken waarin een systematisch literatuuronderzoek staat beschreven naar de lange termijn uitkomsten van patiënten die een IMP programma hebben gevolgd. Deel 2 bevat vier hoofdstukken die betrekking hebben op de ontwikkeling van terugvalpreventie interventies.

\section{Deel 1}

Hoofdstuk 2 en 3 bevatten de volgende onderzoeksvragen:

- Hoe verandert de fysieke, psychische en sociale gezondheid van patiënten met chronische pijn die hebben deelgenomen aan een revalidatieprogramma over tijd?

- In hoeverre variëren de geïncludeerde studies in design, patiëntkenmerken en behandelprogramma's?

- Op welke manier kan een standaard systematisch literatuuronderzoek doorontwikkeld worden tot een 'living systematic review', om daarmee de snelheid van disseminatie en implementatie van de resultaten te bevorderen?

Hoofdstuk 2 bevat de resultaten van een systematisch literatuuronderzoek en metaanalyse. Het primaire doel was om alle gepubliceerde studies te vinden die lange-termijn 
uitkomsten beschrijven van mensen die een IMP programma hebben gevolgd. Er zijn in totaal 65 studies geïdentificeerd die longitudinale uitkomsten hebben gerapporteerd van 74 patiëntcohorten. Voor ieder cohort is de ontwikkeling over tijd in kaart gebracht door verschilscores te berekenen tussen de meting voorafgaand aan de revalidatie (pre), na afloop van de revalidatie (post) en de laatst gemeten follow-up meting. De meeste contrasten tussen de pre- en postmeting (85\%) wezen op een gunstige verandering op diverse uitkomstmaten, waaronder fysiek functioneren, depressie en ervaren beperkingen in het dagelijks leven. Dit positieve effect werd in de meeste gevallen volgehouden tijdens follow-up. De belangrijkste conclusie is dat deelname aan pijnrevalidatie geassocieerd is met positieve veranderingen in gezondheidsuitkomsten, zowel direct na afloop van de revalidatie als op de lange termijn. Hierbij moet worden opgemerkt dat ondanks het gunstige groepseffect de mogelijkheid van terugval voor patiënten zeker niet is uitgesloten. Vervolgonderzoek met individuele patiëntdata kan hier een nauwkeuriger beeld over geven. Verder toonde de beschrijvende analyse van de gebruikte studiedesigns en behandelprogramma's grote variatie in opzet tussen de studies. Dit duidt niet alleen op verschillende visies over hoe pijnrevalidatie moeten worden vormgegeven en onderzocht, maar het belemmert ook een betekenisvolle vergelijking tussen deze programma's.

Een cruciale uitdaging voor systematisch literatuuronderzoek is om bij te blijven met de toenemende hoeveelheid nieuwe studies die iedere dag worden gepubliceerd. Gedegen literatuuronderzoek kan jaren in beslag nemen voordat de uitkomsten beschikbaar zijn, waardoor de resultaten in veel gevallen al bij publicatie achterlopen op de nieuwste inzichten. Om dit probleem tegen te gaan is het concept 'living systematic review' geïntroduceerd. Kenmerkend voor dit studie design is de vaste cyclus waarin de bestaande resultaten continu worden geüpdatet. Ook bevat deze aanpak vaak semiautomatische procedures om onderdelen van het review proces te versnellen. Hoewel living systematic reviews steeds populairder worden is de toepassing vooral bekend in snel ontwikkelende onderzoeksvelden, zoals bij onderzoek naar COVID-19. In dit hoofdstuk staat beschreven hoe deze systematiek ook kan worden toegepast in de context van revalidatie, waarin er in een tragere cyclus van 6 tot 12 maanden iteratief gewerkt wordt aan een steeds grotere bewijslast rondom vooraf afgebakende thema's.

\section{Deel 2}

In Deel 2 van dit proefschrift staat de ontwikkeling van terugval preventie interventies centraal. Hoofdstuk 4-7 gaan over de volgende onderzoeksvragen:

- Wat zijn de korte en lange termijn effecten van generieke zelfmanagement interventies voor patiënten met chronische musculoskeletale pijn op fysiek functioneren, eigen effectiviteit, pijn intensiteit en fysieke activiteit? 
- In welke mate variëren de generieke zelfmanagement interventies in opzet en in gebruikte gedragsveranderingstechnieken om zelfmanagement te ondersteunen?

- In welke mate ondersteunt co-design de totstandkoming van een terugval preventie interventie op basis van de inzichten en ervaringen van belanghebbenden?

- Hoe evalueren patiënten en gezondheidszorgprofessionals het huidige werkboek prototype in de context van pijnrevalidatie met betrekking tot toepasbaarheid en acceptatie?

- Hoe kan de effectiviteit van de digitale applicatie 'AGRIPPA' geëvalueerd worden in termen van kosteneffectiviteit?

- Hoe kan er inzicht verkregen worden in de betrokkenheid en gebruiksvriendelijkheid van patiënten die de AGRIPPA applicatie hebben ontvangen?

Deel 2 start met een systematisch literatuuronderzoek en meta-analyse naar generieke zelfmanagement interventies voor patiënten met chronische musculoskeletale pijn (hoofdstuk 4). Generieke zelfmanagement interventies bieden patiënten scholing om te leren omgaan met de impact van chronische pijn op hun dagelijks leven. Deze aanpak is interessant in de context van terugval preventie en zou elementen kunnen bevatten die van toegevoegde waarde zijn binnen IMP programma's. Het doel van deze studie was om de effectiviteit van generieke zelfmanagement programma's ten opzichte van controle groepen in kaart te brengen met betrekking tot fysiek functioneren, eigen effectiviteit, pijn intensiteit en fysieke activiteit. Er zijn 20 artikelen gevonden waarin de uitkomsten van generieke zelfmanagement programma's binnen gerandomiseerd onderzoek met controlegroep stonden beschreven. Voor iedere relevante uitkomstmaat per studie zijn bij zowel de nameting als de follow-up meting verschilscores berekend tussen de zelfmanagement groep en de controlegroep. Daarnaast zijn de resultaten per uitkomstmaat gepoold, mits er sprake was van voldoende methodologische en statistische overeenkomsten tussen de studies. Bij fysiek functioneren en pijn intensiteit was er bij de postmeting een statistisch significant, maar klinisch niet relevant effect ten gunste van de zelfmanagement groep ten opzichte van de controlegroep. Analyse van de follow-up metingen toonde een statistisch significant effect maar klinisch niet relevant effect voor eigen effectiviteit, ten gunste van de zelfmanagement groep. De overige vergelijkende analyses waren niet statistisch significant. In lijn met de uitkomsten van hoofdstuk 2 was er ook in deze set van studies sprake van substantiële variatie in de opzet van de zelfmanagementprogramma's. Dit was in het bijzonder het geval bij de gerapporteerde gedragsveranderingstechnieken die in de programma's waren verweven. Hieruit blijkt dat er verschillend gedacht wordt over hoe zelfmanagement het beste kan worden ondersteund in dit soort programma's.

Hoofdstuk 5 bevat de beschrijving en systematische evaluatie van een co-design project om een terugval preventie interventie te ontwikkelen voor patiënten die een IMP programma 
succesvol hebben afgerond. De unieke eigenschap van co-design is dat - in tegenstelling tot traditionele interventie ontwikkelingsprojecten - eindgebruikers en andere stakeholders structureel worden betrokken tijdens iedere fase van het ontwikkelingsproces. Het gevolg is dat deze methode nauw aansluit bij de behoeften en wensen van patiënten en zorgprofessionals, wat de kans op succesvolle implementatie verhoogd. Het eerste doel van deze studie was om een overzicht te geven van alle ontwerpactiviteiten die hebben plaatsgevonden tijdens de ontwikkeling van een prototype interventie. Het tweede doel was om per ontwerpfase te reflecteren op de rol van co-design ten aanzien van het genereren van nieuwe inzichten, het betrekken van stakeholders, en het verwerken van stakeholder input in het uiteindelijke ontwerp. In deze zogeheten 'embedded case study' hebben de onderzoekers gebruik gemaakt van participatief actieonderzoek, waarbij ze niet alleen hebben geobserveerd, maar actief hebben samengewerkt met patiënten, zorgprofessionals, studenten en ontwerpers bij de ontwikkeling van een prototype interventie. Tijdens dit project hebben de onderzoekers een kwalitatieve dataset opgebouwd met behulp van notities, resultaten van ontwerpsessies, notulen en reflectieve bijeenkomsten. De ontwerpactiviteiten hebben gedurende 18 maanden plaatsgevonden en bestonden uit interviews, co-creatie sessies, data-analyse bijeenkomsten en het bouwen van prototypes. Tijdens deze activiteiten werd er geleidelijk een kennisbasis opgebouwd, en zijn er twee prototype interventies ontwikkeld die uiteindelijk verwerkt zijn tot één terugval preventie werkboek. De belangrijkste conclusie was dat de generatieve technieken geholpen hebben bij het verkrijgen van een gedetailleerd beeld van ervaringen van deelnemers. De co-design activiteiten stimuleerden ook actieve deelname en samenwerking tussen alle betrokkenen op gelijkwaardige basis. De belangrijkste beperking was de beperkte betrokkenheid van patiënten en zorgprofessionals in de laatste fase van het project, wat mogelijk nadelige gevolgen heeft voor de acceptatie van het uiteindelijke prototype.

De resultaten van de eerste praktijkervaringen met het werkboek staan beschreven in hoofdstuk 6. Het doel van dit kwalitatief onderzoek was om de mate van acceptatie en toepasbaarheid van het werkboek bij zowel patiënten als zorgprofessionals in kaart te brengen. Het werkboek werd beschikbaar gesteld aan twee IMP programma's in twee verschillende revalidatiecentra. Zorgprofessionals kregen een korte training in het gebruik en waren vrij om zelf te kiezen bij welke patiënten ze het werkboek zouden inzetten. $\mathrm{Na}$ afloop van de studiefase hebben we inzichten verzameld met behulp van een focusgroep en diepte interviews, waar 4 zorgprofessionals en 11 patiënten aan hebben deelgenomen. Met behulp van een thematische analyse zijn relevante datasegmenten georganiseerd onder vier hoofdthema's: toepasbaarheid in de huidige behandelsetting, acceptatie van de inhoud van het werkboek, acceptatie van de vorm van het werkboek en aanpassingsvermogen van de interventie aan specifieke patiëntkarakteristieken. Deelnemers vonden het werkboek gemakkelijk in gebruik en goed aansluiten op de behandelcomponenten. Daarnaast werden 
er diverse suggesties voor verbetering van het werkboek en bijbehorende procedures genoemd. Een belangrijke belemmering voor de generaliseerbaarheid was de kleine steekproef en de relatief hoge uitval van deelnemende patiënten. Vervolgstudies zouden daarom niet alleen moeten kijken naar verdere verbetering van de interventie, maar ook goed de studie- en implementatie procedures moeten analyseren.

Een belangrijke suggestie van de haalbaarheidsstudie uit hoofdstuk 6 was om het werkboek te vertalen naar een digitale interventie. In een vervolgproject hebben we de mobiele applicatie AGRIPPA ontwikkeld waarin de belangrijkste principes uit het werkboek prototype waren verwerkt. Er is een educatiemodule aan toegevoegd waar ieder IMP programma eigen informatie aan kon toevoegen, zoals video's, opdrachten en links naar digitale bronnen. In Hoofdstuk 7 staat het protocol beschreven van een gerandomiseerd onderzoek met controlegroep, waarmee de (kosten-)effectiviteit van de applicatie in kaart wordt gebracht. De hoofdvraag was in hoeverre de ontwikkeling over tijd van de interventiegroep verschilde van de controlegroep met betrekking tot ervaren beperkingen in het dagelijks leven. Het doel van deze studie is om inzicht te krijgen in hoeverre de applicatie in staat is om het volhouden van succesvolle behandeluitkomsten te ondersteunen.

Het laatste hoofdstuk bevat de discussie van dit proefschrift. Een belangrijke aanbeveling is een meer nauwkeurige beschrijving van interventies in primaire studies, zodat er een vollediger beeld ontstaat van de variatie in behandel- en studiekarakteristieken. Verder wordt er ingegaan op de potentie van digitale applicaties binnen IMP programma's. De belangrijkste implicaties voor het onderzoeksveld zijn de mogelijkheid van een living systematic review en de toepassing van zelfmanagement interventies, al dan niet als onderdeel van IMP programma's. Verder wordt er stilgestaan bij technocratische en democratische waarde van co-design in interventie ontwikkelingsprogramma's. Tot slot staan er drie concrete suggesties beschreven voor vervolgonderzoek: verdere disseminatie van de AGRIPPA interventie, integratie van gedragsveranderingsprincipes in het proces van klinisch redeneren en het belang van interdisciplinaire publieke consortia ter bevordering van translationeel onderzoek. 



\section{DANKWOORD (ACKNOWLEDGEMENTS)}

Als eerste wil ik mijn promotieteam bedanken voor alle ondersteuning en begeleiding in de afgelopen jaren. Rob, wat ben ik dankbaar voor je oprechte betrokkenheid bij alle facetten van dit proefschrift. Ik heb heel fijn met je samengewerkt en ik kon op ieder moment een beroep op je doen voor persoonlijke begeleiding, inhoudelijke feedback, onderzoeksactiviteiten, co-design sessies, en interviews voor studentenprojecten. Daarnaast heb ik veel gehad aan je kritische blik, je wetenschappelijke én je klinische ervaring die enorm hebben bijgedragen aan de kwaliteit van het onderzoek en de artikelen.

Harriët, vanaf het eerste gesprek in de catacomben van de Bolognalaan 101 heb je me vertrouwen en vrijheid gegeven om met dit onderzoeksproject aan de slag te gaan. Ik heb ontzettend gewaardeerd dat kwaliteit en inhoudelijke argumenten voor jou ten allen tijde leidend zijn geweest bij belangrijke beslissingen. Ook als dit voor de zoveelste keer betekende dat ik een paar extra weken nodig had voor het uitwerken van een analyse. Daarnaast bewonder ik je onnavolgbare werktempo, waarbij ik na het opsturen van manuscripten eindelijk even rust hoopte te hebben, en ze vervolgens dezelfde avond retour kreeg. Ik denk ook met veel genoegen terug aan de pijncongressen die we hebben bezocht en waarin je me geïntroduceerd hebt aan je internationale netwerk. Deze congressen zijn stuk voor stuk hoogtepunten voor mij geweest in de afgelopen jaren.

Jan, ik kijk met een grote glimlach terug op de vele co-creatie sessies die we samen hebben bijgewoond. Het was voor ons allebei een behoorlijke cultuur shock toen we voor het eerst een conceptspace inliepen, die volhing met system maps, prototypes en vooral heel veel postits. Ik vrees dat we onze onervarenheid met deze vorm van onderzoek in eerste instantie moeilijk hebben kunnen verhullen, maar ik bewonder je snelle aanpassingsvermogen en nieuwsgierigheid naar deze vorm van onderzoek. Ik ben je daarnaast ontzettend dankbaar voor de adviezen en je betrokkenheid bij mijn ontwikkeling als onderzoeker. De overlegsessies in de vroege ochtend hielpen om focus te houden op de hoofdzaken en ik kon altijd bij je terecht voor overleg en reflectie.

Ik wil de leden van de beoordelingscommissie, voorzitter Prof. Jeanine Verbunt, Dr. Brigitte Brouwer, Prof. Geert Crombez, Dr. Brona Fullen, en Prof. Madelon Peters bedanken voor de aandacht en zorgvuldigheid waarmee ze mijn proefschrift hebben gelezen en beoordeeld. Prof. Michael Nicholas and Dr. Ivan Huijnen, thank you very much for participating in the defence committee. 
Otto en Jonas, de gedachte dat jullie straks achter me zitten tijdens de promotieceremonie geeft me nu al vertrouwen. Otto, in de gesprekken die we de afgelopen jaren hebben gevoerd over onderzoek vond je steeds weer originele invalshoeken of nieuwe gedachten waar ik veel aan heb gehad. Daarnaast hebben onze sportmomenten zeker geholpen om niet in mijn stoel vast te groeien. Ik bewonder je spontane levenshouding, die me heeft geholpen zo nu en dan uit mijn onderzoeksbubbel te komen. Jonas, onze vriendschap gaat niet alleen al ruim 30 jaar terug, onze levensloop toont opvallend veel gelijkenissen. Of het nu ging om een middelbare school, studiestad, masteropleiding of promotietraject, steeds weer bleek jouw keuze mij ook goed te passen. Bedankt voor het nemen van al mijn belangrijke beslissingen. Ik kijk uit naar de vele avonturen die we ongetwijfeld nog samen gaan beleven. Na Zdorovie!

Dit proefschrift was niet tot stand gekomen zonder de bijdragen van patiënten, collega's, vrijwilligers en zorgverleners. Ik wil iedereen bedanken die tijdens één van de vele cocreatie sessies, interviews, of focusgroepen heeft bijgedragen aan de resultaten van dit onderzoek.

Albère, de vierde musketier van mijn promotieteam! Volgens mij ben je bij praktisch iedere onderzoeksactiviteit uit dit proefschrift betrokken geweest. Een hoogtepunt hierin was het spontane idee om een bus te regelen voor de studenten co-design om hun prototypes in de centrale hal bij Hoensbroek te testen. Jouw kritische onderzoekersblik en je praktijkervaring in de revalidatie vormde een gouden combinatie, waar ik veel aan heb gehad bij het opzetten van onderzoek en schrijven van artikelen. De avond in een bar in Savona beloofd veel goeds voor de keer dat ik echt carnaval met je kom vieren.

Sophie, wat ben jij een geweldige steun geweest bij de tweede systematic review. Toen ik je vroeg voor dit project had ik niet kunnen bevatten hoeveel werk hierin zou gaan zitten. Ik heb met veel plezier met je samengewerkt tijdens alle avond- en weekendsessies waarin we samen duizenden studies hebben doorgespit en besproken. Je hebt zelfs op strategische momenten je partner Juul ingezet bij het programmeren van het dashboard. Je energie, positiviteit, maar vooral ook nauwkeurigheid waren onmisbaar om dit mega project tot een goed einde te brengen. Ik wil je daar ontzettend voor bedanken en ik hoop dat we nog veel blijven samenwerken.

Dear Ulrike, it is an honour to join forces in the quest for improving interdisciplinary pain treatment. I am very grateful for the informative discussions and your help with the review. It is always a real pleasure to exchange new ideas and insights with you. 
Beste Jos, hartelijk dank voor het delen van je expertise en ervaring met systematic reviews. De skype sessies hielpen me richting geven aan het proces en hebben zelfs geleid tot het uitwerken van het idee van een 'living systematic review'.

Else, ik bewonder de manier waarop je het SOLACE project hebt doorgezet in AGRIPPA. Je hebt je in deze periode echt ontwikkeld tot expert van de applicatie en weet als geen ander wat de mogelijkheden hiervan zijn. Ik hoop dat we in de toekomst nog mooie stappen gaan zetten.

Bij een groot deel van de studies in dit proefschrift is er intensief samengewerkt met het lectoraat co-design. Het systemische, ontwerpgerichte perspectief dat zij meebrachten is essentieel geweest voor de ontwikkeling van de interventies. Christa, Remko, Roos, Jens, Rosa en Anita, bedankt! Christa, bij aanvang van SOLACE had ik nooit verwacht hoe groot de omvang van dit project zou gaan worden. Na de eerste vergadering werd een 3 maanden co-design traject al verlengd tot 18 maanden. Daarna volgde onder andere maandenlang ontwerpen in een antikraak pand op Hoog Catharijne, trips naar Maastricht voor interviews, co-creatie sessies en de begeleiding van ruim 60 studenten tijdens een minorproject. Er ging een wereld voor mij open! Verder bewonder ik je gave in het stellen van de goede vragen op het juiste moment. Dit is cruciaal geweest voor de uiteindelijke kwaliteit van het onderzoek. Rosa, jouw 'co-design a pancake' is voor mij hét symbool geworden van de kracht van ontwerpgericht onderzoeken. Wat mooi dat we inmiddels collega's zijn geworden en dat we veel blijven samenwerken. Remko, in jouw rol als facilitator lukte het je steeds weer om deelnemers met totaal verschillende achtergronden en perspectieven productief samen te laten werken. In deze sessies voelden zij zich niet alleen gehoord, maar kregen ze ook echt de ruimte om persoonlijke ervaringen te delen die cruciaal waren voor dit project.

Reint Jan en Sander, ik wil jullie bedanken voor de goede gesprekken en ideeën rondom gedragsverandering in de revalidatie. Het was een enorme luxe om waar nodig te kunnen bouwen op jullie expertise op dit vlak.

Christiaan en alle collega's van DIO Design Agency, de snelheid waarmee jullie een papieren werkboek hebben omgetoverd tot een prachtige applicatie was echt indrukwekkend. Dank voor de goede samenwerking.

Ik wil ook de collega's van de pijnrevalidatie Adelante, locatie Hoensbroek en Maastricht bedanken voor het delen van hun expertise en inzichten over hun programma's. Marlies, in het bijzonder bedankt voor de meeloopdagen, waarop ik me weer een paar dagen behandelaar voelde. 
Hoewel ik de laatste jaren weinig in Maastricht hoefde te zijn heb ik me altijd zeer welkom gevoeld bij de vakgroep revalidatiegeneeskunde. Margareth, bedankt voor alle ondersteuning en planning. Inge, Reni, Marlies, Mariëlle, Laura, Vera, Thijs, Cynthia en Ivan, bedankt voor de goede gesprekken en gezellige momenten in Maastricht en op de pijncongressen.

Alle (oud-)collega's van het Lectoraat Leefstijl en Gezondheid: Martine, Edwin, Han, François, Else, Janke O., Sabrine, Manon, Marike, Imke, Jacqueline N, Marleen, Eline, Janke G, Barbara, Ryan, Richard, Karlijn, Michiel, Henri, Jacqueline O., Hannelies, Claudia en Sonja. Ontzettend bedankt voor de samenwerking, de koffiemomenten en het lotgenotencontact in de afgelopen jaren. Marike, even koffiedrinken met jou leidt vaak tot levendige gesprekken over onderzoek, sport, of verbouwen. Het was leuk om gelijk met je op te lopen en regelmatig hoogte- en dieptepunten uit te wisselen. Bedankt voor je goede adviezen. Manon, je hebt me de afgelopen tijd een mooi inzicht gegeven in wat er allemaal komt kijken bij het opzetten en organiseren van onderzoeksprojecten. Het is echt indrukwekkend om te zien wat je allemaal voor elkaar krijgt. Daarnaast kijk ik iedere keer weer uit naar onze gezamenlijke workshops gedragsverandering en dan met name naar de voorbereidingssessies in The Village of bij Cornelis. Janke, je hebt me op sleeptouw genomen in de eerste jaren van mijn promotietraject. Dankjewel voor het delen van al je inzichten en ideeën. Ook de volksdans en whisky ervaring in Edinburg zal ik niet snel vergeten. Martine, Han en Edwin, wat hebben we toch een mooi vak! Bedankt voor alle inspirerende gesprekken, gezamenlijke lessen en hulp in de afgelopen jaren.

Alle collega's van het kenniscentrum gezond en duurzaam leven: bedankt voor de goede samenwerking in diverse projecten en de goede sfeer op de Heidelberglaan. Marc, jouw hulp met $\mathrm{R}$ was absoluut onmisbaar bij de totstandkoming van de systematic review. Dankjewel voor alle support en je aanstekelijk enthousiasme voor programmeren in R. Jurgen, ik wil je ontzettend bedanken voor je nauwkeurige begeleiding bij mijn eerste review.

IBS collega's: Jullie stonden altijd klaar om mee te denken in focus groepen, om nieuwe prototypes uit te proberen, maar ook om taken over te nemen op piekmomenten. Bedankt! Ik weet niet of ik echt dankbaarheid voel voor de vrijdagochtend trainingssessies, maar door Jeroen, Inge, Fedde, Mohamed, Peter C, Peter G, Allard, Ronald en de rest van de ochtendtrainingsgroep weet ik in ieder geval waar mijn fysieke grenzen liggen en wat het begrip nederigheid inhoudt. Peter, jaren geleden heb je mij als docent, na een matige praktijktoets, dringend geadviseerd om verder te kijken dan een zesje. Daar ben ik je nog steeds dankbaar voor. Je passie voor het vak en je ideeën rondom embodiment zijn erg aanstekelijk. Ik hoop dat we nog veel gaan samenwerken op dit vlak. Fedde, Norman, Sijmen, Stefan J, Allard, Selma, dank voor alle inspirerende gesprekken en gezamenlijke 
lessen in de afgelopen jaren. Monja, jouw snelle initiatief om mij in 2015 te koppelen aan het lectoraat was feitelijk de eerste stap in dit hele traject. Dankjewel voor het meedenken en vertrouwen!

PSF collega's Linda, Mieke, Ariane, Janke, Esther, Sophie, Annet en Evelien: ontzettend bedankt voor de inspirerende vrijdagen en het leuke team. Linda, ik heb veel waardering voor je betrokkenheid en je hulp bij het zoeken naar een goede balans tussen onderzoek en onderwijs. Evelien, wat was het fijn om de afgelopen drie jaar intensief met je samen te werken. Ik kijk met veel plezier terug op alle lessen die we verzorgd hebben en ik heb veel geleerd van je expertise en visie over psychosomatiek. Ook kon ik altijd op je rekenen als ik het overzicht kwijt was in drukke tijden. Bedankt!

Leden van de Pain Community, bedankt voor de leuke bijeenkomsten en het delen van jullie expertise.

Laura, Daphne, Teuntje, Sophie, Deloryan, Rianne, Yasmijn, Myrthe, en alle andere studenten die een bijdrage hebben geleverd aan dit onderzoeksproject: ontzettend bedankt!

Tijdens piekmomenten was het regelmatig belangrijk om stoom af te blazen of gedachten te verzetten. Ik wil bij deze mijn vrienden bedanken voor de fietstochten, beachvolleybal sessies, bootcamp en boardcamp avonden en concertbezoeken. Tom, je scherpe en nuchtere kijk hebben me steeds weer geholpen bij het vinden van het juiste perspectief en bij het maken van belangrijke keuzes. David, de muzieklokaal sessies waren voor mij een soort ideale vorm van intervisie. Ik blijf graag met je van gedachten wisselen over het combineren van fysiotherapie en psychologie. Olivia, de hardloop- en boksbal sessies, vakanties, Belinfante repetities en wandelingen zijn slechts een kleine selectie van de vele gezamenlijke lichtpunten tijdens de afgelopen coronajaren. Ik kijk uit naar nieuwe hoofdstukken. Maurice, ik zie je veel te weinig, maar wat is het fijn dat ik altijd op je kan rekenen. Fedde en Inge, de Pandemic avonden tijdens de pandemic waren een groot succes. Laten we de draad snel weer oppakken want er staan nog twee seizoenen op ons te wachten.

Lieve Teeja en Willem, ik voel me bevoorrecht met jullie onvoorwaardelijke steun en vertrouwen. Ik vind het heerlijk om na een intensieve periode weer bij te tanken en afstand te nemen bij jullie in Nijmegen. Met een glas wijn aan de eettafel vliegen de uren vaak voorbij. Wat fijn dat jullie zo meeleven met alle ups- en downs en dat ik altijd terecht kan voor goede gesprekken en reflectie. 
Lieve Pieter, Charlotte en Alex, bedankt voor alle bijzondere momenten die we al samen hebben meegemaakt. Of we nu in Parijs aan een terras zitten, rondlopen in Berlijn, of samen zijn op de veldstraat, het is altijd direct gezellig en ontspannen. Ik kijk ontzettend uit naar wat we allemaal nog samen gaan beleven.

Lieve Wilma en Paul, ook jullie hebben veel bijgedragen aan wie ik nu ben. Een bezoek aan Nijmegen voelt eigenlijk niet compleet zonder een borrel bij jullie op het terras. Bedankt voor alle mooie momenten samen!

Lieve Guus en Prunella, Driebergen voelt inmiddels al jaren als een derde thuis. Ik kijk altijd uit naar heerlijke zelfgemaakte taart, potjes tafeltennis of de live muziek op de achtergrond. Guus, je unieke kijk op het leven leiden niet zelden tot interessante inzichten. Ik waardeer onze gesprekken, klusmomenten en je ongedwongen adviezen over al mijn onderzoeks- en verbouwingsbeslommeringen.

En tot slot, lieve Rosina, wil ik jou bedanken voor al je geduld en vertrouwen. Dit project heeft veel moois opgeleverd, maar jij hebt als geen ander ook de perioden van frustratie, onderzoeksmanie en twijfel meegekregen. Keer op keer had je begrip en hielp je me relativeren. Met jou kan ik de wereld aan. Met jou ben ik gelukkig. 




\section{CURRICULUM VITAE}

Stefan Elbers, born on May 22th 1987, graduated in 2005 from the Stedelijk Gymnasium Nijmegen. After obtaining his bachelor's degree Physiotherapy in 2009 he combined a parttime position as physiotherapist at the University Medical Center Utrecht with a bachelor's degree Psychology at Utrecht University. Inspired by the scientific culture in both locations, he enrolled in the research master program Social and Health Psychology and was appointed as teacher applied behavioural science at the University of Applied Sciences Utrecht. He completed an internship in the self-regulation lab of Utrecht University, where he explored the relationship between trait self-control and the ability to form desirable or break undesirable habits in the context of health behaviours. After obtaining his master's degree in 2014, he was offered a position as researcher in the Lifestyle and Health research group of the University of Applied Sciences in Utrecht. From 2015 to 2019 he worked on several research projects that centred on preventing relapse after successful pain rehabilitation. From 2019 onwards, Stefan co-authored various awarded research grant applications, including the SIA RAAK Publiek PAIN research program that he currently supervises with Dr. Harriët Wittink. As researcher-consultant, he also regularly collaborates in research projects that involve behaviour change. Stefan currently combines his research with a teaching position at the institute for human movement studies. His main activities include lectures on health behaviour regulation and teaching at the Master of Science program in psychosomatic physiotherapy. Since 2021, Stefan also became member of the EFIC Academy Board and the IASP taskforce on interdisciplinary pain treatment. 



\section{LIST OF GRANTS, PUBLICATIONS AND PRESENTATIONS}

\begin{tabular}{ll}
\hline Research Grants \\
\hline 2020 & RAAK public grant (Raak.Pub06.014) 'Pain therapy in primary care'. (co-author). \\
2018 & $\begin{array}{l}\text { RAAK top-up grant (Raak 2014-01-23). 'Increasing awareness of co-design methodology for the } \\
\text { development of healthcare interventions' (co-author). }\end{array}$ \\
2016 & HU PhD grant. Funding to work on a PhD project from 2016 to 2020. \\
\hline
\end{tabular}

Publications in peer reviewed journals

2021 Elbers, S., Wittink, H., Konings, S., Kaiser, U., Kleijnen, J., Pool, J., Köke, A., \& Smeets, R. (accepted unedited manuscript). Longitudinal outcome evaluations of interdisciplinary multimodal pain treatment programs for patients with chronic primary musculoskeletal pain: a systematic review and meta-analysis. European journal of pain.

Elbers, S., van Gessel, C., Renes, R. J., van der Lugt, R., Wittink, H., \& Hermsen, S. (2021). Innovation in Pain Rehabilitation Using Co-Design Methods During the Development of a Relapse Prevention Intervention: Case Study. Journal of medical internet research, 23(1), e18462.

2020 Elbers, S., Pool, J., Wittink, H., Köke, A., \& Smeets, R. (2020). Exploring the Feasibility of Relapse Prevention Strategies in Interdisciplinary Multimodal Pain Therapy Programs: Qualitative Study. JMIR human factors, 7(4), e21545.

Elbers, S., Pool, J., Wittink, H., Köke, A., Scheffer, E., \& Smeets, R. (2020). Mobile health app (AGRIPPA) to prevent relapse after successful interdisciplinary treatment for patients with chronic pain: Protocol for a randomized controlled trial. JMIR research protocols, 9(8), e18632.

2018 Elbers, S., Wittink, H., Pool, J., \& Smeets, R. The effectiveness of generic self-management interventions for patients with chronic musculoskeletal pain on physical function, self-efficacy, pain intensity, and physical activity: A systematic review and meta-analysis. European Journal of Pain, 22(9), 1577-1596.

\section{Other publications}

2021 Book chapter: Verdonk, B. Heerkens, Y,. Elbers, S., \& Damstra, R. Terminologie en zorgmodellen voor mensen met chronische aandoeningen. In Verdonk, Devoogdt \& Damstra (Eds.), Oedeem en oedeemtherapie (pp. 79-87). Houten: Bohn stafleu van loghum

2018 Article: Elbers, S., Hermsen, S., Bloemen, M., Renes, R., \& Wittink, H. Behavioral Lenses, theory and application. Published in Fysiopraxis (Dutch journal for physiotherapist).

2017 Article: Elbers, S. The psychology of pain. Published in Think Big: honors magazine of the social sciences faculty at Utrecht University.

2010 Article: Elbers, S., Verdonk, B., El Ouamari, M., Holtslag, H., \& Aufdemkampe, G. Exploring the effect of passive mobilization of the MTP joint on the venous flow in the lower limb region: a pilot study. Published in Oedeminis in March 2010. 


\section{Selection of lectures, workshops and presentations}

2021 Presentation: 'Longitudinal outcome evaluations of interdisciplinary multimodal pain treatment programs: results of a living systematic review’ at the Scherzkongress 2021 in Mannheim.

Workshop Chair: Self-management and chronic pain’ of the EFIC Virtual Education Summit 2021.

Podcast appearance: at Smartbeat podcast episode 5: 'Lifestyle and applied behavioural science’.

Podcast appearance: at Physio Global podcast episode 1: 'Successful behaviour change in patients: reality or fiction'.

Podcast appearance: at Trajectum podcast episode: 'Why walking and running can be equally beneficial for your health'.

2020 Presentation: 'Goal-setting in chronic pain treatment', at the EFIC virtual Pain Summit.

Presentation: 'Interdisciplinary chronic pain treatment: time for a Paradigm Shift?, at the VU Musculoskeletal Science Group symposium.

Workshop: 'Into the Unknown: New perspectives on training, pain and physical activity, at the Fyveau conference

2019 Guest lecture: 'Clinical reasoning in the context of relapse prevention and behavior change', in the module chronic musculoskeletal pain for the Physician specialist education program in rehabilitation medicine.

Presentation: 'Theory and application of the persuasive by design model within physiotherapy', at the 'physical activity for children with a disability' symposium at the University of Applied Sciences Utrecht.

Presentation: 'Beyond motivation: an extended perspective on behavior regulation in the context of physical exercise programs', at the national physical education teachers conference 'Move to the next level'.

Poster presentation: 'Preventing Relapse after Successful Rehabilitation: A Feasibility Study of Two Prototype Interventions' at the EFIC conference in Valencia (Spain)

Poster presentation: 'Innovation in pain management: How co-design can guide the development of a complex intervention' at the Pain Science in Motion conference in Savona (Italy)

Guest Lecture: 'Clinical reasoning and behavior change' at the FH Joanneum (physiotherapy education) in Graz (Austria).

2018 Poster presentation: 'Preventing relapse after successful treatment: A feasibility study of two prototype interventions' at the IASP conference in Boston.

2017 Presentation: 'Evaluating co-design methodology in the development of an intervention' at the EFIC conference in Copenhagen.

Poster presentation: 'Effectiveness of self-management interventions' at the EFIC conference in Copenhagen.

Poster presentation: 'How to prevent relapse after successful pain rehabilitation?' at the Pain Science in Motion conference in Stockholm.

Workshop: 'Behavior change and physical activity in children with a disability’ with Dr. M. Bloemen at the 'children and motor development' symposium at the university of applied sciences Utrecht.

Presentation: 'Solace: Preliminary results' during the SOLACE symposium at the university of applied sciences in Utrecht. 
Selection of lectures, workshops and presentations

2016 Workshop: 'How to live a healthy life with chronic pain: Self-regulation and self-management in elderly with chronic pain' at the conference of the national association of geriatric physiotherapy (NVFG).

Presentation: 'Tailoring behavior change interventions' at the national conference of the National Physiotherapy Association (KNGF).

Workshop series (5x): for the Dutch National Physiotherapy Association (KNGF) 'Guidelines physical activity and chronic pain’ with Dr. H. Wittink, Dr. A. Köke and J. Oosterhaven, MSc.

2015 Guest lecture: ‘The Psychology of Touch’ at the BSc psychology program at Utrecht University. 